Thomas Märtz

\title{
Interessengruppen und Gruppeninteressen in der Demokratie
}




\section{Interessengruppen und Gruppeninteressen in der Demokratie}

Die Theorie des Rent-Seekingstellt einen Erklärungsansatzfür das Zustandekommen staatlicher Aktivitäten in demokratischen Gesellschaftssystemen dar. Im Rahmen einer politisch-ökonomischen Analyse werden die allokativen und distributiven Konsequenzen des Wettbewerbs von Interessengruppen um gruppenspezifische Verteilungsvorteile untersucht. Dabei wird insbesondere auf die Interdependenz von distributiven Gruppeninteressen und politischem Entscheidungsverhalten eingegangen. Zur Lösung des Verteilungsdilemmas werden Reformvorschläge für den politischen Entscheidungsprozeß entwickelt, die eine Neuorientierung der Ordnungspolitik in demokratischen Staatswesen implizieren.

Thomas Märtz wurde 1959 in Heidelberg geboren. Von 1979 bis 1985 Studium der Wirtschaftswissenschaften an der Universität Hohenheim. Seit Februar 1985 Wissenschaftlicher Mitarbeiter bei Prof. Dr. C. Folkers am Lehrstuhl für Finanzwissenschaft der Universität Hohenheim. 
Interessengruppen und Gruppeninteressen in der Demokratie Zur Theorie des Rent-Se日king 


\section{Hohenheimer Volkswirtschaftlicheschriften}

Herausgegeben von

Prof.Dr. Cay Folkers, Prof.Dr. Joachim Genosko, Prof.Dr.Harald Hagemann, Prof. Dr. Klaus Herdzina, Prof.Dr.Franz Mehlert, Prof.Dr. Renate Ohr, Prof.Dr. Walter Piesch, Prof.Dr.Ingo Schmidt, Prof.Dr. Helmut Walter, Prof.Dr. Josua Werner,

Band 12

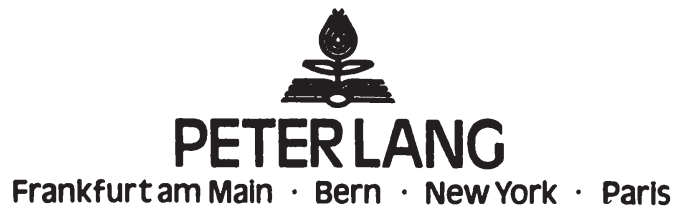




\section{Thomas Märtz}

\section{Interessengruppen und Gruppeninteressen in der Demokratie Zur Theorie des Rent-Seeking}

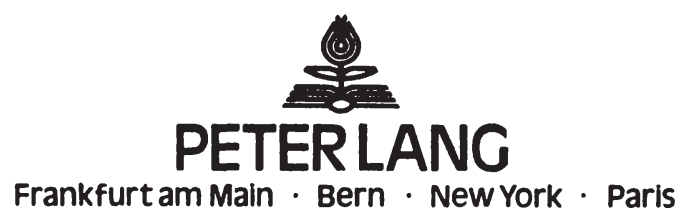




\title{
CIP-Titelaufnahme der Deutschen Bibliothek
}

Mārtz, Thomas:

Interessengruppen und Gruppeninteressen in der Demokratie : Zur Theorie des Rent-Seөking / Thomas Mårtz. - Frankfurt am Main ; Bern ; New York ; Paris : Lang, 1990

(Hohenheimer volkswirtschaftliche Schriften ; Bd. 12)

Zugl.: Hohenheim, Univ., Diss., 1989

ISBN 3-631-42685-2

NE: GT

Open Access: The online version of this publication is published on www.peterlang.com and www.econstor.eu under the international Creative Commons License CC-BY 4.0. Learn more on how you can use and share this work: http:// creativecommons.org/licenses/by/4.0.

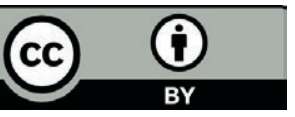

This book is available Open Access thanks to the kind support of ZBW - Leibniz-Informationszentrum Wirtschaft.

\author{
D 100 \\ ISSN 0721-3085 \\ ISBN 3-631-42685-2 \\ ISBN 978-3-631-75470-2(eBook) \\ (C) Verlag Peter Lang GmbH, Frankfurt am Main 1990 \\ Alle Rechte vorbehalten.
}

Das Werk einschlieBlich aller seiner Teile ist urheberrechtlich geschützt. Jede Verwertung außerhalb der engen Grenzen des Urheberrechtsgesetzes ist ohne Zustimmung des Verlages unzulässig und strafbar. Das gilt insbesondere fūr Vervielfăltigungen, Übersetzungen, Mikroverfilmungen und die Einspeicherung und Verarbeitung in elektronischen Systemen.

\section{Printed in Germany 124567}




\section{Geleitwort}

Die wachsende Zahl begünstigender staatlicher Regulierungen, Subventionen und Steuerermäßigungen wird zunehmend als zentrales Problem für die Funktionsfähigkeit marktwirtschaftlicher Prozesse erkannt. Diese Maßnahmen führen nicht nur zu allokativen Wohlfahrtsverlusten und distributiven Ungerechtigkeiten, sondern bewirken auch Anreize, Ressourcen aus produktiven Verwendungen in die Erzeugung und Verteidigung von staatlichen Regelungen zu investieren, die politisch garantierte Renten versprechen. Die allokativen und distributiven Implikationen dieses Ressourceneinsatzes im Wettbewerb der Interessengruppen stehen im Mittelpunkt eines neuen ökonomischen Forschungsansatzes, für den sich die Bezeichnung "Theorie des Rent-Seeking" eingebürgert hat. Der Ansatz erklärt die Entstehung staatlicher Institutionen aus dem ökonomischen Rationalverhalten der begünstigten Individuen bzw. Gruppen und trägt zu einem tieferen Verständnis der Voraussetzungen und Konsequenzen begünstigender Regelungen bei.

Die vorliegende Arbeit enthält eine umfassende Analyse des theoretischen Konzepts und eine kohärente Würdigung der bisher in der Literatur vorliegenden, stark divergierenden Einzelansätze. Sie entwickelt ein allgemeines Modellkonzept des Rent-Seeking und wendet es auf Fragen der Gewährung von Verteilungsvorteilen durch finanzwirtschaftliche Maßnahmen des Staates an. Sowohl aus der theoretischen Grundsatzdiskussion als auch aus der Anwendung des gedanklichen Konzepts auf ein zentrales Problem der Finanzpolitik ergeben sich zahlreiche neue Erkenntnisse zur aktuellen Problematik der Interessenpolitik.

Die Arbeit entstand während der Tätigkeit des Verfassers als Wissenschaftlicher Mitarbeiter im Rahmen des langfristigen Forschungsprogramms des Lehrstuhls für Finanzwissenschaft der Universität Hohenheim. Sie eröffnet als Teil einer erst in der Entwicklung begriffenen ökonomischen Forschungsrichtung vielfältige Ansätze für weiterführende Analysen und politische Grundsatzdiskussionen. Im Hinblick auf die Bedeutung der behandelten Fragen ist zu hoffen, daß die vorliegende Schrift sowohl der wissenschaftlichen als auch der politischen Diskussion wirksame Impulse vermittelt. 
Thomas Märtz - 978-3-631-75470-2

Downloaded from PubFactory at 01/11/2019 04:38:03AM

via free access 


\section{Vorwort}

Die vorliegende Dissertation entstand im Rahmen meiner Tätigkeit als Wissenschaftlicher Mitarbeiter am Lehrstuhl für Finanzwissenschaft der Universität Hohenheim. Mein besonderer Dank gilt meinem Doktorvater Prof. Dr. Cay Folkers, der den Anstoß zu dieser Untersuchung gab und die Arbeit mit wertvollen Anregungen und kritischen Hinweisen förderte. Ebenso bin ich ihm für die Gewährung eines Druckkostenzuschusses aus Spendenmitteln des Lehrstuhls zu Dank verpflichtet.

Meinem Kollegen Dipl.-Volkswirt Peter Mendler danke ich für zahlreiche ermunternde Diskussionen, die zur Durchdringung wesentlicher Problembereiche beigetragen haben. Für die kollegiale Zusammenarbeit sei Dipl.oec. Sabine Novak, Dr. Friedhelm Herb sowie Dipl.oec. Karin Beckmann gedankt.

Danken möchte ich gleichfalls meinen Eltern, die mich im Laufe meiner Ausbildung in vielfältiger Weise unterstützt haben. Meine Frau Ulrike begleitete die Entstehung dieser Arbeit mit großem Verständnis und erstellte mit Geduld und Sorgfalt die endgültige Fassung des Manuskriptes. Nicht nur dafür danke ich ihr ganz herzlich. 
Thomas Märtz - 978-3-631-75470-2

Downloaded from PubFactory at 01/11/2019 04:38:03AM

via free access 
I. EINLEITUNG 1

1. Methodische Grundlagen 6

2. Gang der Untersuchung 9

II. DEFINITION UND ABGRENZUNG DES RENT-SEEKING 11

1. Das Konzept der ökonomischen Rente 12

2. Der Wettbewerb um Renten 15

2.1. Profit-Seeking 15

2.2. Rent-Seeking 18

3. Die Entscheidung zwischen Profit-Seeking 27 und Rent-Seeking

4. Profit-Seeking und Rent-Seeking aus
institutionelier Sicht

5. Zusammenfassung 34

III. DIE GRUNDSTRUKTUR DER RENT-SEEKING-ARGUMENTATION 36

1. Das Grundmodell des Rent-Seeking 37

2. Das Modell des kompetitiven Rent-Seeking 42

2.1. Der Wettbewerb um Monopolprivilegien 42

2.2. Der Wettbewerb um Monopolprivilegien unter Berücksichtigung von Unsicherheit

3. Allokative Konsequenzen des Rent-Seeking 56

4. Interpretation und Kritik der Rent-Seeking-
Konzeption

5. Zusammenfassung 70 
1. Interessengruppen und fiskalische Gruppeninteressen

2. Interessengruppen als Produzenten von Verteilungsvorteilen

2.1. Bestimmung des gruppeneffizienten Produktionsniveaus von politischem Einfluß

2.2. Bestimmung der gruppeneffizienten Produktionstechnik

3. Die Konsequenzen der gruppeninternen Interessenkonstellation

4. Die Bedeutung der Gruppenfunktionäre

5. Zusammenfassung

V. DER WETTBEWERB VON INTERESSENGRUPPEN UM POLITISCHEN EINFLUSS

1. Die Interessenantinomie beim Distributionskonflikt

2. Die Handlungsmöglichkeiten der Gruppenmitglieder

2.1. Die Effekte marktlicher Anpassungsreaktionen

2.2. Die Effekte politischer Einflußnahmeaktivitäten

3. Das Gleichgewicht der Distributionsinteressen

4. Die Effekte einer Veränderung der Determinanten des Distributionsgleichgewichts

4.1. Steigerung der Produktionseffizienz politischen Drucks

4.2. Veränderung der Mehrbelastung

4.3. Veränderung der Gruppengröße

5. Interpretation und Kritik der Ergebnisse

6. Die Konsequenzen marktmäßiger Verteilungseffekte

7. Zusammenfassung 
VI. GRUPPENINTERESSEN UND POLITISCHES ENTSCHEIDUNGSVERHALTEN IN DER DEMOKRATIE

1. Die Funktionsweise des politischen Entscheidungssystems

1.1. Die Nachfrage nach staatlichen Institutionen

1.2. Das Angebot an staatlichen Institutionen

2. Distributionsinteressen und Entwicklung der Staatstätigkeit

3. Gruppeninteressen in der Mehrheitsdemokratie

3.1. Mehrheitswahlsystem und staatliche Umverteilung

3.2. Die Durchsetzung von Sonderinteressen im Vergünstigungsstaat

3.2.1. Informationsbeeinflussung und Wählerverhalten

3.2.2. Lobbying und Politikerverhalten

3.2.3. Stimmentausch

3.3. Die Interessen der Bürokratie

4. Interessengruppen und Zunahme der Staatstätigkeit

4.1. Das Grundmodell

4.2. Die Effekte einer begünstigenden Steuerstruktur

4.3. Die Effekte einer begünstigenden Ausgabenstruktur

4.3.1. Das Verhalten der begünstigten Gruppe

4.3.2. Das Verhalten der oppositionellen Gruppe

4.4. Interpretation und Kritik der Ergebnisse 
VII. DAS DILEMMA DES POLITISCHEN INTERESSENWETTBEWERBS

1. Die Anreizstruktur beim Wettbewerb unter marktlichen Institutionen

2. Die Anreizstruktur beim Wettbewerb unter politischen Institutionen

2.1. Rent-Seeking im Rechtsschutzstaat

2.2. Rent-Seeking im Vergünstigungsstaat

3. Zusammenfassung

VIII. ZUR REFORM DER "RENT-SEEKING-SOCIETY"

1. Zur Neuorientierung der Ordnungspolitik

2. Rent-Seeking aus konstitutioneller Perspektive

3. Ansatzpunkte für institutionelle Reformen

3.1. Begrenzung politischer Handlungsspielräume

3.2. Förderung marktlicher Aktivitäten

3.3. Realisierungschancen und Implementationsprobleme

4. Zusammenfassung

IX. SCHLUSSBEMERKUNGEN 
Seite

\section{VERZEICHNIS DER ABBILDUNGEN}
Abbildung 1: Das Konzept der ökonomischen Rente
Abbildung 2: Die Entscheidung zwischen Rent-Seeking und Profit-Seeking
Abbildung 3: Die sozialen Kosten des Wettbewerbs um Monopolprivilegien
Abbildung 4: Rent-Seeking und gesellschaftliche Produktionsmöglichkeiten
Abbildung 5: Die Effekte des Ressourceneinsatzes für Lobbying
Abbildung 6: Die Kosten und Nutzen politischer Einflußnahme
Abbildung 7: Die Entscheidung zwischen alternativen Einflußnahmetechniken
Abbildung 8: Die Mehrbelastungseffekte von Steuern und Vergünstigungen
Abbildung 9: Die gruppenspezifische Einflußnahme
Abbildung 10: Das Gleichgewicht der Distributions- interessen
Abbildung 11: Die Effekte von Parametervariationen auf das Distributionsgleichgewicht
Abbildung 12: Die allokativen Konsequenzen des Wett- bewerbs um politischen Einfluß
Abbildung 13: Die Übertragung von Distributionsvorteilen
Abbildung 14: Die Wirkungen einer interessenbedingten Informationsbeeinflussung 105 
VERZEICHNIS DER ÜBERSICHTEN

Übersicht 1: Profit-Seeking versus Rent-Seeking

Übersicht 2: Die Funktionsweise des politischen Entscheidungssystems

Übersicht 3: Zur Neuorientierung der Ordnungspolitik

\section{VERZEICHNIS DER TABELLEN}

Tabelle 1: Individueller Ressourceneinsatz beim Wettbewerb um eine Monopolrente

Tabelle 2: Gesellschaftlicher Ressourceneinsatz beim Wettbewerb um eine Monopolrente

Tabelle 3: Ausgaben und Einnahmen des Staates

Tabelle 4: Symmetrische Interessenstruktur beim Profit-Seeking

Tabelle 5: Symmetrische Interessenstruktur beim Rent-Seeking im Rechtsschutzstaat

Tabelle 6: Asymmetrische Interessenstruktur beim Rent-Seeking im Vergünstigungsstaat 
"L'État, c'est la grande fiction à travers laquelle tout le monde s'efforce de vivre aux dépens de tout le monde."

Frédéric Bastiat (1854) 
Thomas Märtz - 978-3-631-75470-2

Downloaded from PubFactory at 01/11/2019 04:38:03AM

via free access 


\section{EINLEITUNG}

Seit einiger Zeit hat das Interesse an Institutionen in der wissenschaftlichen Diskussion wieder an Bedeutung gewonnen. Gegenstand der Institutionenanalyse ist die Untersuchung von Funktionsweise, Wirkungen, Entwicklung und Gestaltung wirtschaftlicher und gesellschaftlicher Institutionen. Im Zuge der "institutionalistischen Revolution"1) werden auch der ökonomischen Theorie neve Impulse vermittelt. Das systematische Verständnis realer Phänomene in modernen Wirtschafts-und Gesellschaftssystemen setzt eine Analyse der wechselseitigen Beziehungen zwischen Institutionen und ökonomischen Aktivitäten von Individuen bzw. gesellschaftlichen Gruppen voraus. Aufgrund der Anwendungsbreite des institutionellen Ansatzes existiert bisher kein einheitliches Theoriegebilde, sondern eine Reihe einzelner Forschungsrichtungen, die unter dem Begriff "New Institutional Economics" ${ }^{2}$ ) zusammengefaßt werden.

Die Renaissance der Institutionenanalyse führt zu einer Wiederbelebung der politischen Auseinandersetzung über die Funktionsbedingungen von marktwirtschaftlichen Wirtschaftsordnungen. Die immer deutlicher werdende Erkenntnis, daß sich permanente politische Interventionen bei der Lösung von wesentlichen wirtschaftspolitischen Problemen als weitgehend wirkungslos erwiesen haben, wirft die Frage nach alternativen Strategien auf. Die in den letzten beiden Jahrzehnten entwickelte Theorie des Rent-Seeking analysiert das Zustandekommen staatlicher Aktivitäten im politisch-ökonomischen Zusammenhang.

1) Albert (1977), S. 203.

2) Vgl. dazu Furubotn/Richter (1984), S. 1 ff., Coase (1984), S. 229 ff., Williamson (1985a), S. 187 ff. bzw. (1985b) und North (1986), S. $230 \mathrm{ff}$. 
Im Mittelpunkt ordnungstheoretischer Kontroversen steht die Problematik der Legitimation staatlichen Handelns in Marktwirtschaften ${ }^{1)}$. Als zentrales Argument für die Begründung staatlicher Aktivitäten im Allokationsbereich dient das Konzept des Marktversagens ${ }^{2}$ ). Kann gezeigt werden, daß der Marktprozeß aufgrund des Vorliegens bestimmter Bedingungen ein soziales Optimum verfehlt, wird die Schlußfolgerung gezogen, der Staat solle zur Korrektur des Marktversagens intervenieren. Ausgehend von der grundlegenden Allokationsproblematik der Bereitstellung öffentlicher Güter wurden zahlreiche weitere staatliche Eingriffe mit einem Marktversagen begründet. Theoretische Voraussetzung für Aktivitäten des Staates muß allerdings der Nachweis sein, daß der politische Prozeß ein Resultat hervorbringt, das dem Marktergebnis allokativ überlegen ist.

In Verbindung mit Fragen der Verteilungsgerechtigkeit werden häufig Idealvorstellungen entwickelt, um staatliche Maßnahmen zur Korrektur der sich aus dem Marktprozeß ergebenden Einkommensverteilung zu rechtfertigen. Auch in diesem Bereich muß untersucht werden, inwieweit das Ziel der Verteilungsgerechtigkeit über politische Prozesse zu verwirklichen ist, d.h. es muß ein Vergleich der Institutionen "Markt" und "Staat" vorgenommen werden ${ }^{3)}$. Verteilungszielsetzungen bestimmen den Umfang und die Struktur des Steuersystems, äußern sich in der Gewährung von Subventionen bzw. Transferzahlungen und finden in zahlreichen gesetzlichen Regelungen zur Absicherung und zum Schutz einzelner Individuen oder bestimmter gesellschaftlicher Gruppen gegenüber sich wandelnden marktlichen Verhältnissen ihren Niederschlag.
1) Vgl. z.B. Windisch (1980), S. $297 \mathrm{ff}$.
2) Vgl. Bator (1958), S. $351 \mathrm{ff}$.
3) Vgl. Brennan/Buchanan (1981), S. $103 \mathrm{ff}$. 
Allerdings weisen die im politischen Prozeß festgelegten staatlichen Aktivitäten starke Widersprüchlichkeiten auf. So finden sich beispielsweise Hilfen für die Landwirtschaft neben Unterstützungen für die Stahlindustrie, werden Forschungsmittel für sogenannte Zukunftsindustrien und Erhaltungssubventionen für "alternde" Industrien vergeben bzw. Subventionen für den Zonenrand gleichzeitig mit Förderprogrammen zugunsten von Ballungsgebieten beraten ${ }^{1)}$. Diese Unsystematik staatlicher Interventionen läßt die Vermutung zu, daß zahlreiche Aktivitäten des Staates weniger auf die Verwirklichung bestimmter sozialer Normen gerichtet als vielmehr auf den Einfluß gruppenspezifischer Interessen zurückzuführen sind. Neben dem Sozialstaatsprinzip werden meist Effizienzargumente ${ }^{2}$ ) zur politischen Rechtfertigung herangezogen, um das eigentliche Umverteilungsmotiv in den Hintergrund zu rücken.

Die Existenz vieler staatlicher Aktivitäten mit Umverteilungscharakter wirft die Frage nach den Ursachen von Distributionsprozessen und nach den an ihrem Zustandekommen beteiligten Faktoren auf. Damit ergibt sich die Notwendigkeit der Entwicklung einer positiven Theorie öffentlicher Redistributionstätigkeit, welche die konkrete Form realer Umverteilungsphänomene erklären und bestimmte Gesetzmäßigkeiten bei der Entstehung staatlicher Regelungen aufzeigen kann.

1) Eine Klassifikation der zahlreichen staatlichen Interventionen nach Wirtschaftszweigen erfolgt bei Gerstenberger/ Heinze/Hummel/Sprenger (1984), S. $176 \mathrm{f}$. aber auch bei Thormählen (1987), S. 388 f. Einen Überblick über die vielfältigen Regulierungen und Subventionen zugunsten einzelner Branchen gibt der Sachverständigenrat zur Begutachtung der gesamtwirtschaftlichen Entwicklung im Jahresgutachten $(1988 / 89)$, S. 149 .

2) Hier sei beispielhaft auf die immer wiederkehrende Forderung nach einer aktiven Industriepolitik bzw. nach staatlicher Forschungsförderung verwiesen. 
Die Theorie des Rent-Seeking ${ }^{1)}$ untersucht die Interdependenz von distributiven Gruppeninteressen und politischem Entscheidungsverhalten in demokratischen Gesellschaftssystemen. Gemäß dieser theoretischen Konzeption sind die zahlreichen Interventionen des Staates das Ergebnis der politischen Konkurrenz um Distributionsvorteile.

Mit dem Schlagwort einer "Rent-Seeking-Society"2) wird eine Gesellschaft bezeichnet, die ihre Ressourcen zunehmend in die Verteidigung von Besitzständen, in die Erlangung von staatlichen Garantien und in die Absicherung von vielfältigen politisch geschützten Monopolrenten investiert. Individuen werden versuchen, diese Vorteile durch aktive Einflußnahme auf den politischen Entscheidungsprozeß zu verteidigen oder zu erweitern. Inwieweit sich Verteilungsvorteile zugunsten einzelner Gruppen in der erfolgreichen Durchsetzung bestimmter Staatsausgaben, Steuergesetze bzw. administrativer Regulierungen niederschlagen, wird entscheidend von den Möglichkeiten der Partizipation am politischen Entscheidungsprozeß und damit von der existierenden Verteilungssituation bestimmt. Die Mitglieder der Gesellschaft können am Distributionskonflikt teilnehmen, wobei es vom Organisationsgrad abhängt, ob und in welchem Umfang spezielle Interessen im politischen Prozeß berücksichtigt werden. Daraus ergibt sich die Schlußfolgerung, daß in demokratischen Systemen Interessengruppen um die gruppenspezifisch begünstigende Ausgestaltung institutioneller Bedingungen - und damit um die Gestaltung des Ordnungsrahmens des Wirtschaftssystems - konkurrieren.

1) Eine zusammenfassende Darstellung der Theorie des RentSeeking gibt Tollison (1982).

2) Neben der Bezeichnung "Rent-Seeking-Society" findet man ebenfalls den Begriff "Transfer-Society". Vgl. dazu Rowley/Tollison/Tullock (1988) und Anderson/Hill (1980) bzw. Thurow (1980). 
Mit der Betonung von Distributions- und Machtaspekten bei der Entstehung und Ausgestaltung statlicher Aktivitäten rückt der Rent-Seeking-Ansatz die Beziehung zwischen allokativen und distributiven Zielsetzungen der Statstätigkeit in das Licht kritischer Betrachtung, da bei allen redistributiv motivierten staatlichen Maßnahmen die Auswirkungen auf die Ressourcenallokation einbezogen werden müssen.

Während beim traditionellen Ansatz eine separate und neutrale Ausrichtung staatlicher Aktivitäten gemäß den Erfordernissen der Allokations-bzw. Distributionsfunktion ${ }^{1}$ ohne wechselseitige Beeinflussungen vorgesehen ist, liegt beim RentSeeking-Konzept eine Vermengung beider Funktionen des öffentlichen Sektors vor. Der zentrale Unterschied besteht darin, daß allokative Maßnahmen des Staates mit distributiven Zielen begründet werden.

Eine gesellschaftliche Gruppe kann ihre ökonomische Position verbessern, wenn sie im politischen Prozeß eine für sie günstige Regelung durchsetzt. Diese Möglichkeit induziert Anreize, Einkommen durch politische Entscheidungen und zunehmend weniger durch Marktprozesse zu realisieren. Die Theorie des Rent-Seeking lenkt die Aufmerksamkeit auf die Gruppeninteressen bei Verteilungsfragen und stellt einen Erklärungsansatz für die Entstehung vielfältiger regulierender Maßnahmen, für die permanente redistributiv motivierte Umgestaltung des Steversystems und für die Gewährung zahlreicher Transferprogramme in modernen Wohlfahrtsstaaten dar ${ }^{2}$ ).

1) Vgl. dazu insbesondere Musgrave (1974), S. 3 ff. Gemäß der "Multiplen Theorie des öffentlichen Haushaltes" hat der Staat neben der Allokations- und Distributionsfunktion auch eine Stabilisierungsfunktion zu erfüllen.

2) Vgl. Gäfgen (1984), S. 55 ff. bzw. Anderson (1987), S. $377 \mathrm{ff}$. 


\section{Methodische Grundlagen}

Im Rahmen der Theorie des Rent-Seeking wird die Entstehung staatlich determinierter Institutionen auf das rationale Verhalten von Individuen zurückgeführt. "The analysis of rent seeking is, therefore, properly designed as institutional economics in a very real sense" ${ }^{1)}$. Der traditionelle neoklassische Ansatz erfährt eine institutionelle Ergänzung, indem Entscheidungen über staatliche Institutionen selbst zum Gegenstand der ökonomischen Analyse werden. Damit weist das Konzept des Rent-Seeking enge Beziehungen zur Theorie der PropertyRights und dem Forschungsansatz der Public-Choice-Theorie auf ${ }^{2}$.

Als theoretische Grundlage der Untersuchung dient der methodologische Individualismus ${ }^{3}$, der die individuellen Handlungen in den Mittelpunkt der Betrachtungen stellt. Gemäß dem "ökonomischen Ansatz" 4 ) individuellen Handelns wird von einem rationalen Verhalten der Wirtschaftssubjekte bei Vorliegen stabiler Präferenzen ${ }^{5}$ ) ausgegangen.

1) Buchanan (1980a), S. 14. In ähnlicher formulierung vgl. North (1987a), S. 163 bzw. (1987b), S. 419 ff.

2) "Rent seeking analysis can readily be incorporated within the property-rights approach and, as with public choice, the theory of rent seeking can be interpreted as an appropriate extension." Buchanan (1980a), S. 15. Vgl. dazu auch Benson (1984).

3) So konstatieren Riker/Ordeshook (1973), S. 34, daß "we cannot find a principle to explain the preferences and choices of society other than derive them from people's preferences and choices." Zum Begriff des methodologischen Individualismus vgl. Buchanan/Tullock (1962), S. $11 \mathrm{ff}$.

4) Vgl. Becker (1976), S. $6 \mathrm{f}$.

5) Zur Bedeutung der Annahme stabiler Präferenzen für ökonomische Analysen vgl. den Beitrag "De Gustibus Non Est Disputandum" von Stigler/Becker (1977), S. 76 ff. bzw. Tietzel (1988a), S. 38 ff. 
Ein Individuum wird in einer Entscheidungssituation diejenige Handlungsalternative wählen, mit der sich die höhere Rente erzielen läßt. Der Begriff der ökonomischen Rente bezeichnet den Überschuß des Ressourceneinsatzes über die Opportunitätskosten, d.h. über die Erträge in alternativer Verwendung ${ }^{1)}$.

Aufgrund ihrer verhaltenssteuernden Eigenschaften kommt der Analyse von Institutionen zentrale Bedeutung zu. Institutionen $^{2}$ ) stellen festgelegte Regeln für das Entscheiden von Individuen in sich wiederholenden Entscheidungssituationen dar und beeinflussen die Handlungsalternativen der Wirtschaftssubjekte. Damit bilden Institutionen die Rahmenbedingungen, innerhalb derer Individuen handeln und wechselseitige Beziehungen entfalten. Neben individuellen Verhaltensreaktionen bestimmen die institutionellen Bedingungen auch die Einkommensund Vermögensverteilung ${ }^{3)}$ einer Gesellschaft.

Im Rahmen der ökonomischen Analyse von Institutionen lassen sich im wesentlichen zwei Fragestellungen ${ }^{4}$ unterscheiden. Zum einen kann das individuelle Verhalten unter gegebenen Institutionen (choice within rules) untersucht werden. Dieser Problembereich beinhaltet den Vergleich der ökonomischen Wirkungen exogen vorgegebener Institutionen, die durch die individuellen Anpassungsreaktionen hervorgerufen werden. Aus dieser Perspektive werden Institutionen als Parameter angesehen, die alternative, exogen festgelegte Ausprägungen annehmen.

1) Vgl. z.B. Tollison (1982), S. 577 .

2) Zur Problematik der Definition des Institutionenbegriffs vgl. Vanberg (1983), S. $55 \mathrm{ff}$. bzw. auch Elsner (1986), S. $199 \mathrm{ff}$. Zum Begriff der "sozialen Institutionen" sei auf Schotter (1981), S. 11 verwiesen.

3) Vgl. North (1988), S. 17 .

4) Vgl. zu dieser grundlegenden Unterscheidung insbesondere Buchanan (1967), S. 213 ff. 
Auf der anderen Seite kann der Frage nachgegangen werden, inwieweit Institutionen ihrerseits durch individuelles Verhalten beeinflußt werden können (choice of rules). Bei dieser Fragestellung werden die Institutionen als endogene Variablen behandelt, deren Zustandekommen und Ausgestaltung sowie deren Ausgestaltungsänderungen zu untersuchen sind.

In traditioneller Sicht werden staatliche Regulierungseingriffe oder Regelungen im Bereich der Steuer- und Ausgabenpolitik als Institutionen begriffen, an die sich Individuen mit ihren marktmäßigen Entscheidungen anpassen. Die Beschränkung der Analyse auf reine Anpassungsreaktionen an staatliche Maßnahmen bedeutet eine willkürliche Einschränkung der individuellen Handlungsmöglichkeiten, da Individuen durch politische Aktivitäten eine begünstigende Änderung staatlicher Institutionen anstreben können. Um beide Dimensionen individuellen Handelns erfassen zu können, ist die überwindung der bisherigen Trennung von ökonomischen und politischen Mechanismen erforderlich.

Im Rahmen der Analyse des Rent-Seeking wird die Problematik des Entscheidungsverhaltens unter gegebenen Institutionen in Verbindung mit der Möglichkeit einer gezielten Änderung der institutionellen Bedingungen zur Realisierung individueller Distributionsvorteile betrachtet. Das Zustandekommen staatich determinierter Institutionen wird aus dem Zusammenwirken von marktmäßigen Effekten einzelner staatlicher Maßnahmen und der interessenbedingten Einflußnahme von Individuen bzw. Interessengruppen auf die jeweilige Ausgestaltung erklärt. Dabei ist von Bedeutung, inwieweit die gegebenen politischen Institutionen der Demokratie die Realisierung von Verteilungsvorteilen zugunsten von Sonderinteressen zulassen. 


\section{Gang der Untersuchung}

Der Rent-Seeking-Ansatz wird im folgenden zur Entwicklung einer positiven Theorie statlicher Distributionspolitik verwendet. Zu diesem Zweck müssen die ökonomischen und politischen Einflußfaktoren der Bestimmung und Veränderung staatlich determinierter Institutionen untersucht werden, um aus ihrem Zusammenwirken im politischen Entscheidungsprozeß Erklärungen für Art und Umfang staatlicher Maßnahmen abzuleiten.

Den Ausgangspunkt der Analyse bilden die Definition und Abgrenzung des Untersuchungsgegenstandes. Nach der Darstellung des ökonomischen Rentenkonzeptes werden alternative Formen des Wettbewerbs um Renten diskutiert. Das grundlegende Modell des Rent-Seeking wird in Verbindung mit der Problematik von regulierenden Interventionen des Staates zugunsten einzelner Wirtschaftssubjekte bzw. bestimmter Wirtschaftszweige entwickelt. Die Zielsetzung der Interessenten besteht in der Schaffung spezieller Schutzbereiche und der Verteidigung dieser Privilegien vor unerwünschter marktlicher Konkurrenz. Im Rahmen des Modells des kompetitiven Rent-Seeking werden die Determinanten des individuellen und gesellschaftlichen Ressourceneinsatzes beim Akquisitionswettbewerb um staatlich gewährte "Monopolprivilegien" herausgearbeitet. In diesem Zusammenhang werden insbesondere die Allokationswirkungen einer erfolgreichen Durchsetzung begünstigender Ausnahmeregelungen von den allgemeingültigen wettbewerblichen Institutionen diskutiert.

Die Einflußnahme auf die Ausgestaltung staatlicher Institutionen ist ein charakteristisches Merkmal der Aktivitäten von Interessengruppen. Zur Beurteilung der Konsequenzen einer interessenbezogenen Beeinflussung von politischen Entscheidungen werden die Bestimmungsfaktoren der Bildung von Interessengruppen und ihre Verhaltensweisen bei der Durchsetzung der gemeinsamen Gruppeninteressen untersucht. 
Die Analyse widerstreitender Verteilungsinteressen erfolgt auf der Grundlage eines Gleichgewichtsmodells, wobei die konkurrierenden Bemühungen von Interessengruppen zur Gewinnung von politischem Einfluß die Höhe der gruppenspezifischen Distributionsvorteile bestimmen. Dabei wird insbesondere auf die Beziehungen zwischen den marktmäßigen Anpassungsreaktionen der Gruppenmitglieder an fiskalische Institutionen und der interessenbedingten Einflußnahme auf die Ausgestaltung der institutionellen Bedingungen eingegangen.

Staatliche Institutionen werden jedoch nicht von Interessengruppen ausgehandelt, sondern im politischen Prozeß festgelegt. Aus diesem Grund ist weiterhin zu untersuchen, inwieweit das Zustandekommen institutioneller Entscheidungen durch die gegebenen politischen Institutionen in demokratischen Systemen beeinflußt wird. Im Mittelpunkt der Analyse steht dabei die Interdependenz $z$ wischen den Forderungen organisierter gesellschafticher Gruppen und dem resultierenden politischen Entscheidungsverhalten. Auf dieser Grundlage werden die Konsequenzen des Verteilungswettbewerbs von Interessengruppen für den Umfang des öffentlichen Sektors in marktwirtschaftlichen Wirtschaftsordnungen aufgezeigt.

Im Rahmen einfacher spieltheoretischer Überlegungen werden die unterschiedlichen Interessenkonstellationen beim Wettbewerb über den marktlichen und den politischen Sektor dargestellt. Dabei wird der Frage nachgegangen, unter welchen institutionellen Bedingungen ein individuell rationales Verhalten zu gesellschaftlich wünschenswerten Resultaten führt. Die Arbeit schließt mit der Entwicklung von Reformvorschlägen für das politische Entscheidungsverfahren, die eine Neuorientierung des ordnungspolitischen Denkens in demokratischen Staatswesen implizieren. 


\section{DEFINITION UND ABGRENZUNG DES RENT-SEEKING}

Die Theorie des Rent-Seeking setzt sich mit der grundlegenden Problematik des individuellen Strebens nach Renten auseinander. Dabei stehen insbesondere die institutionellen Bedingungen für das individuelle Verhalten bei der Suche nach Renten im Vordergrund $^{1)}$. Das Ziel der individuellen Nutzenmaximierung kann sowohl unter den gegebenen institutionellen Bedingungen des Marktprozesses (Profit-Seeking) als auch durch eine vorteilhafte Veränderung staatlich determinierter Institutionen über den politischen Entscheidungsprozeß (Rent-Seeking) verfolgt werden. Aus der Rent-Seeking-Perspektive wird damit explizit die Möglichkeit berücksichtigt, durch politische Einflußnahme begünstigende Maßnahmen in Gestalt administrativer Regulierungseingriffe, Transferregelungen bzw. steuerlicher Strukturen ${ }^{2}$ zu realisieren und damit spezifische Distributionsvorteile zu erringen.

Ausgehend vom Konzept der ökonomischen Rente werden zunächst alternative Formen des Wettbewerbs um Rentenpositionen betrachtet. Danach wird auf die Entscheidung zwischen Profit-Seeking und Rent-Seeking eingegangen. Anschließend werden aus institutioneller Perspektive die wesentlichen methodischen Unterschiede zwischen beiden Ausprägungen des Wettbewerbs herausgearbeitet. Die Verlagerung des Wettbewerbs vom marktlichen in den politischen Sektor ${ }^{3}$ ) induziert Anreize zur Durchsetzung von staatlichen Aktivitäten, die ihrerseits die Entstehung weiterer politischer Regelungen mit vornehmlich distributiven Zielsetzungen bedingen. Den Abschluß dieses Kapitels bildet eine Zusammenfassung der gewonnenen Resultate.

1) "Any theory of rent-seeking must ultimately be a theory of institutions." Michaels (1988), S. 27.

2) Vgl. Folkers $(1986 a)$, S. 8.

3) In diesem Zusammenhang wird auch von einer "Politisierung von Restriktionen" gesprochen. Vgl. Siebert (1985), S. 108. 


\section{Das Konzept der ökonomischen Rente}

Im Rahmen der individuellen Nutzenmaximierung stellt das Streben nach Renten ${ }^{1)}$ die zentrale Triebfeder ökonomischen Handelns dar. Jedes Individuum verfügt über eine spezifische Ausstattung mit Ressourcen, die zur Durchführung verschiedener Aktivitäten eingesetzt werden können. Zum Zwecke dieser Untersuchung wird ein allgemeiner Ressourcenbegriff gewählt, der neben materiellen und nicht-materiellen Gütern auch personenspezifische Fähigkeiten und Fertigkeiten einschließt ${ }^{2}$ ). Die Verwendung bestimmter Teile der Ressourcenausstattung definiert eine individuelle Handlung.

Aufgrund der Knappheit der Ressourcen ergibt sich die Notwendigkeit zur Entscheidung im Hinblick auf alternative Einsatzmöglichkeiten der zur Verfügung stehenden Ressourcen. Die Wahl einer bestimmten Ressourcenverwendung bedeutet den Verzicht auf potentielle, jedoch konkurrierende Handlungsalternativen. Die Opportunitätskosten der individuellen Entscheidung sind der Ertrag der Ressource in ihrer nächstbesten Verwendungsmöglichkeit ${ }^{3)}$. Da jeder Aktivität eine Abwägung der Nutzen und Kosten der Konsequenzen vorausgeht, wird sich ein rational handelndes Individuum für diejenige Handlung entscheiden, mit der sich die höchste Rente erzielen läßt. Der Begriff der Rente wird daher als Überschuß über die Opportunitätskosten einer Ressource ${ }^{4}$ ) definiert.

1) Eine zusammenfassende Darstellung des Rentenkonzeptes findet sich bei Currie/Murphy/Schmitz (1971), S. 741 ff.

2) In dieser umfassenden Definition bezeichnet der Ressourcenbegriff alle Faktoren, die ein "Akteur zur Beeinflussung seiner ... Umwelt einsetzen kann". Vanberg (1982), S. 11.

3) Zur Diskussion verschiedener ökonomischer Kostenbegriffe sei auf Buchanan (1969), S. 38 ff. verwiesen.

4) Vgl. Tollison (1982), S. 577 bzw. Buchanan (1980a), S. 3. 
Ausgehend von dieser allgemeinen Rentendefinition können einzelne Rentenformen ${ }^{1)}$ unterschieden werden. Unter "Ricardianischen Renten" versteht man unterschiedliche Erträge für bestimmte Ressourceneinheiten in ihrer gegenwärtigen Verwendung, die gleiche Opportunitätskosten aufweisen. Im Gegensatz dazu wird bei einer gleichen Entlohnung in derzeitiger Verwendung und differierenden Opportunitätskosten der einzelnen Ressourceneinheiten von "Differentialrenten" gesprochen. Während bei Ricardianischen Renten vollständig homogene Ressourceneinheiten unterstellt werden, liegt bei der Variante der Differentialrenten die Vorstellung von heterogenen Einheiten der Ressourcen zugrunde. Da sich das Ressourcenangebot in Abhängigkeit von der Höhe der jeweiligen Opportunitätskosten ergibt, besitzt die entsprechende Angebotsfunktion im ersten (zweiten) Fall einen konstanten (steigenden) Verlauf.

In gleicher Weise können im Hinblick auf die zeitliche Entwicklung des Ressourcenangebots "reine" Renten von sogenannten "Quasi-Renten"2) abgegrenzt werden. Während reine Renten bei kurzfristig konstantem Ressourcenangebot anfallen, bezeichnet der Begriff Quasi-Rente den Tatbestand, daß durch die Ausdehnung des Ressourcenangebots diese Renten langfristig abgebaut werden und eine Entlohnung der marginalen Ressourceneinheit in Höhe der Opportunitätskosten erfolgt. Die grundlegenden Beziehungen werden anhand von Abbildung 1 illustriert ${ }^{3}$.

1) Eine Zusammenfassung und Abgrenzung der zahlreichen Rentenarten findet sich bei Alchian (1987), S. 141 ff.

2) Zum Begriff der Quasi-Rente bzw. der "Composite quasi-rent" vgl. Marshall (1920), S. 74 bzw. S. 626. "When two separately owned resources are so specific to each other that their joint rent exceeds the sum of what each could receive if not used together, then the joint rent to the pair was called 'composite quasi-rent'." Alchian (1987), S. 142 .

3) Vgl. Ekelund Jr./Tollison (1986), S. $326 \mathrm{ff}$. aber auch Browning/Browning (1983), S. $434 \mathrm{ff}$. 


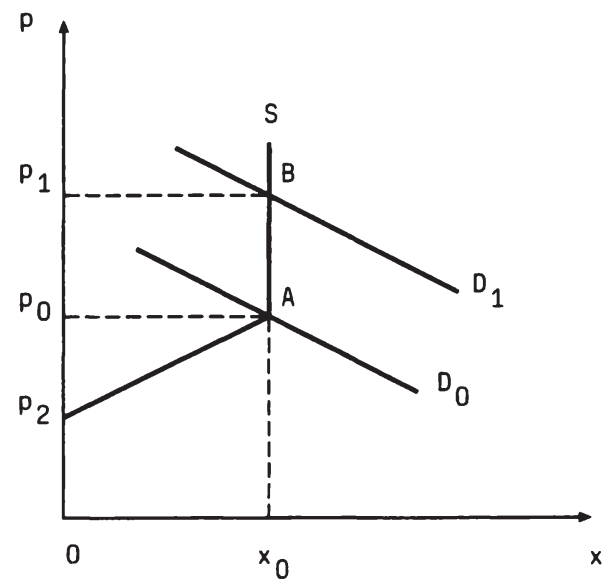

Abb. 1: Das Konzept der ökonomischen Rente

Die Angebotsfunktion $S$ stellt die Opportunitätskosten einer bestimmten Ressourcenmenge $x$ dar. Der Anbieter erhält im Gleichgewicht $A$ Differentialrenten in Höhe von $\mathrm{p}_{0} A p_{2}$. Ergibt sich nun eine exogene Veränderung der Nachfrage nach dieser Ressource (Verschiebung von $D_{0}$ auf $D_{1}$ ) und ist das Angebot bei $x_{0}$ kurzfristig konstant, erhält der Besitzer zusätzlich einen Rentengewinn in Form einer reinen Rente $\left(p_{1} B A p_{0}\right)$. Erfolgt aufgrund der Existenz dieser Renten eine Steigerung des Ressourcenangebots, resultiert eine Verminderung der Erträge der bisherigen Ressourceneinheiten. Das erhöhte Angebot impliziert langfristig eine Rentenerosion, die einen Zustrom von weiteren Ressourcen verhindert. 


\section{Der Wettbewerb um Renten}

Zur Abgrenzung alternativer Formen der Suche nach Renten wird zwischen dem Wettbewerb um Rentenpositionen im Wege des marktlichen Allokationsmechanismus und der Konkurrenz um Renten über den politischen Koordinationsmechanismus unterschieden. Während im Marktprozeß "natürliche" Renten anfallen, werden durch staatliche Aktivitäten sogenannte "politische" Renten geschaffen, um die durch Einflußnahme auf den politischen Entscheidungsprozeß konkurriert werden kann.

\subsection{Profit-Seeking}

Die Konkurrenz um Rentenpositionen im Rahmen des marktlichen Wettbewerbsprozesses wird mit dem Begriff "Profit-Seeking" bezeichnet ${ }^{1)}$. Während in statischer Sicht keine Notwendigkeit für die Existenz ökonomischer Renten besteht, wird ihre zentrale Bedeutung bei dynamischer Betrachtung sichtbar. Die Erzielung von Quasi-bzw. Differentialrenten führt zu einer ständigen Reallokation der Ressourcen in ihre ertragreichste Verwendung. Solange Erträge in Form von Renten als Belohnung für produktive Tätigkeiten - z.B. für innovatorische Aktivitäten im Prozeß der "schöpferischen Zerstörung"2) oder für die Durchsetzung neuer Faktorkombinationen ${ }^{3)}$ - entstehen und nur temporär anfallen, erfüllen sie eine wichtige Funktion in einer evolutorischen ökonomie.

1) Vgl. Buchanan (1978), S. 14. Diese Bezeichnung umfaßt sowohl das Streben nach Konsumentenrenten als auch nach Produzentenrenten.

2) Vgl. Schumpeter (1975), S. 134 .

3) Vgl. Kirzner (1973). 
Bestehen keine Marktzutrittsbeschränkungen, wird die Existenz solcher Gewinne Imitatoren anziehen, deren Verhalten zu einer Rentenerosion bei den Innovatoren führt. Gleichzeitig impliziert die Gefahr von möglichen Verlusten eine Symmetrie des Anpassungsprozesses an sich wandelnde Marktkonstellationen. Das Profit-Seeking-Verhalten bewirkt den Einsatz von Ressourcen zu produktiven Zwecken im Sinne einer Ausweitung des gesamtwirtschaftlichen Output und führt unter bestimmten Bedingungen zu einer Pareto-effizienten Allokation der volkswirtschaftlichen Ressourcen ${ }^{1)}$. Das individuell rationale Streben nach marktlichen Renten ist damit für Wachstum und Entwicklung einer ökonomie von zentraler Bedeutung ${ }^{2}$.

Voraussetzung für die Koordination der separaten Aktivitäten in einer marktwirtschaftlichen Ordnung ist die Existenz institutioneller Bedingungen, die eine übereinstimmung von individuellem Rationalverhalten und gesellschaftlich wünschenswerten Konsequenzen ermöglichen. Entscheidend für das Funktionieren des marktlichen Allokationsmechanismus ist ein System von Eigentumsrechten, das den Individuen erlaubt, Ressourcen zu nutzen bzw. andere Wirtschaftssubjekte von ihrer Nutzung auszuschließen. Individuelle Verfügungsrechte und Vertragsfreiheit begründen die Motivation, wechselseitig vorteilhafte Transaktionen vorzunehmen. Charakteristisch für die institutionellen Bedingungen des Marktprozesses ist insbesondere die Tatsache, daß sie für eine Vielzahl von Individuen gelten - unabhängig von den ökonomischen Ergebnissen, die ihre Anwendung im Einzelfall hervorbringt. Hinzu kommt, daß sie für jedes Wirtschaftssubjekt bestimmte Restriktionen darstellen, die nur beiderseitig gewünschte Aktivitäten zulassen.

1) Vgl. z.B. Sohmen (1976), Kap. 3 und 4 .

2) "Without this personal drive toward profit-seeking, the market economy would simply cease to function." Buchanan (1978), S. 15 . 
Solange die Zuordnung von Eigentumsrechten nicht dem Einfluß der Ressourcenbesitzer unterliegt ${ }^{1)}$, ergibt sich aus dem Zusammenwirken der an ihren eigenen Interessen orientierten Individuen eine "spontane Ordnung"2), die auf dem "Mechanismus der gegenseitigen Anpassung" ${ }^{3}$ ) beruht. Unter den marktlichen Institutionen resultiert als unintendiertes Ergebnis der vielfältigen, auf freiwilliger Basis vorgenommenen, individuell rationalen Aktivitäten ein effizienter Einsatz der volkswirtschaftlichen Ressourcen.

Zur Realisierung der sozial wünschenswerten Ergebnisse sind Umfang und Inhalt der individuellen Eigentums- und Handlungs rechte durch das Rechtssystem zu definieren und zu schützen. Solange die Funktion des States in der Definition der Grenzen legitimer Ausübung privater Handlungsrechte und dem Schutz dieser Rechte besteht, wird er zur unverzichtbaren Voraussetzung für die Entfaltung produktiver Aktivitäten im marktlichen Wettbewerbsprozeß. Da das individuelle Handeln unter den gegebenen marktlichen Institutionen a priori keine daverhaften ökonomischen Gewinne garantiert, ergibt sich ein Anreiz zur "Aushöhlung" dieser Institutionen durch staatlich determinierte Regelungen. Die Wirkungen des Marktmechanismus bilden die Grundlage für die Versuche, mit staatlicher Hilfe Privilegien langfristig zu sichern bzw. marktliche Verteilungsverluste abzuwehren. Mit der Ausdehnung staatlicher Aktivitäten über den protektiven Staat hinaus werden zunehmend Möglichkeiten eröffnet, durch Einflußnahme auf den politischen Entscheidungsprozeß Distributionsvorteile zu realisieren.

1) Vgl. Congleton (1980), S. 154. Zu den eigentumsrechtlichen Mindesterfordernissen des Funktionierens von Marktsystemen vgl. Hayek (1973), S. 285 ff.

2) Vgl. Hayek (1973), S. 37.

3) Vgl. Hayek (1971), S. 192. 


\subsection{Rent-Seeking}

Zur Charakterisierung der Konkurrenz um politisch abgesicherte Rentenpositionen wurde der Begriff "Rent-Seeking"1) geprägt. Die Möglichkeit des Staates, bestimmte individuell vorteilhafte Positionen zu gewähren, macht ihn zum Adressaten zahlreicher Aktivitäten mit distributiven Zielsetzungen. Die erfolgreich durchgesetzten Distributionsvorteile können ebenfalls als Renten interpretiert werden. Im Mittelpunkt der Analyse steht das Verhalten von Wirtschaftssubjekten, die den Staat als Instrument zur Realisierung von Renten benutzen ${ }^{2}$. Untersucht man die existierende Rent-Seeking-Literatur im Hinblick auf eine exakte begriffliche Erfassung und Abgrenzung des Phänomens, sieht man sich einer Vielzahl von Definitionen gegenüber, die eine Klassifikation einzelner Ausprägungen des Untersuchungsgegenstandes erschweren ${ }^{3}$.

Der Begriff "Rent-Seeking-Society" wird anschaulicher, wenn man ihn im Zusammenhang mit der weitgehenden Aufhebung des Prinzips einer spezifischen Entgeltlichkeit für staatliche Leistungen sieht, die bestimmte einzelne Wirtschaftssubjekte oder spezielle gesellschaftliche Gruppen begünstigen.

1) Explizit wurde der Begriff "Rent-Seeking" erstmals von Krueger (1974), S. 291 verwendet.

2) "Modern economists have introduced the term 'rent-seeking' to describe profit seeking behavior within the public sector." Buchanan (1978), S. 16.

3) Die in der Literatur vorzufindenden Begriffsbestimmungen erfassen meist nur Teilaspekte des Rent-Seeking-Phänomens oder gehen bereits von einem das Ergebnis der Analyse determinierenden Begriffsinhalt aus. Eine kritische Zusammenfassung verschiedener Definitionen des Rent-Seeking findet sich bei Samuels/Mercuro (1984), S. 55 f. 
Rent-Seeking-Aktivitäten richten sich auf die Erlangung von Transferleistungen des States in einer weiten Definition, die neben direkten monetären und realen Transfers (im Bereich der sozialen Sicherung) auch Subventionen und Steuervergünstigungen ${ }^{1)}$ umfassen. Hinzu kommen individuell oder gruppenspezifisch begünstigende Leistungen in Bereichen (z.B. Verkehrs-, Bildungs-, Beschäftigungs- oder Wohnungswesen), die unentgeltlich oder zu subventionierten Preisen bereitgestellt werden.

Darüber hinaus haben zahlreiche, nicht direkt an monetäre Größen anknüpfende staatliche Aktivitäten ähnliche Konsequenzen wie Transfers. Die Vielzahl staatlicher Marktregulierungen in Form von Wettbewerbsbeschränkungen auf den Faktormärkten - insbesondere auf dem Arbeitsmarkt - sowie weitere gruppenspezifisch vorteilhafte Regelungen in Form vermögenssichernder Sonderstellungen und Monopolisierungen lassen sich in gleicher Weise als staatliche Umverteilungsmaßnahmen interpretieren ${ }^{2)}$. Alle staatlichen Instrumente dieser Art können selektiv eingesetzt werden und ermöglichen eine Anwendung des Ausschlußprinzips. Der gezielte Einsatz dieser staatlichen Maßnahmen führt zu einer vorteilhaften Distributionsänderung für eine bestimmte Gruppe.

1) Bei der Klassifikation von Steververgünstigungen als implizite Transfers werden nur Ausnahmeregelungen von allgemeinen, der Realisierung der "Steuergerechtigkeit" dienenden Tatbestandsdifferenzierungen erfaßt, d.h. es geht um die speziellen Abweichungen von der allgemeinen Stevernorm. Innerhalb dieser Gruppe staatlicher Transfers erschwert die verwirrende Vielzahl von Bezeichnungen (tax-expenditures, tax preferences, tax exclusions, implicit transfers bzw. tax aids) eine exakte Abgrenzung.

2) Dazu zählen Arbeitszeitvorschriften, Ladenschlußgesetze und zahlreiche weitere begünstigende Berufs-, Arbeitsplatzund Marktrestriktionen. 
Die gruppenspezifische Ausrichtung staatlichen Handelns ermöglicht eine Abgrenzung gegenüber den allgemeinen, in der Regel allen Individuen ${ }^{1)}$ zugute kommenden staatlichen Leistungen ( $z$. $B$. innere und äußere Sicherheit, Rechtswahrung usw.), die zu den legitimen Aufgaben des klassischen protektiven Staates gehören.

Aufgrund seines Machtmonopols ist der Staat in der Lage, Zwang auszü̈ben und damit redistributive Maßnahmen durchzuführen. Mit der Betonung des Machtaspekts als Grundlage staatlicher Umverteilungstätigkeit wird die Problematik angesprochen, inwieweit derart motivierte statliche Aktionen auf individuellen Präferenzen beruhen. Zur Abgrenzung der Rent-SeekingKonzeption soll der zentrale Unterschied zu anderen Erklärungsansätzen staatlicher Umverteilungstätigkeit verdeutlicht werden. Verzichtet man auf die Analyse von Fragen der Verteilungsgerechtigkeit und betrachtet die Distributionsproblematik unter Effizienzaspekten ${ }^{2}$ ), werden individuelle Redistributionsaktivitäten vorwiegend auf Versicherungsmotive ${ }^{3)}$, auf die Existenz von interdependenten Nutzenfunktionen bzw. auf den Kollektivgutcharakter der Verteilung zurückgeführt ${ }^{4}$ ).

1) Allerdings werden diese allgemeinen staatlichen Leistungen eine individuell unterschiedliche Bewertung erfahren.

2) Die Begründung für diese Vorgehensweise liegt in der bekannten Problematik interpersoneller Nutzenvergleiche.

3) Den Grundgedanken des Versicherungsmotivs aus konstitutioneller Perspektive entwickeln Buchanan/Tullock (1962), S. $189 \mathrm{ff}$.

4) Zu einem Überblick einzelner Erklärungsansätze staatlicher Umverteilungstätigkeit vgl. Rodgers (1974), S. $165 \mathrm{ff}$. Vgl. auch Pommerehne (1980), S. 169 ff. 
Im Fall des Versicherungsmotivs wird das individuelle Umverteilungsverhalten in Verbindung mit dem Schutz vor unerwarteten Ereignissen gesehen. Grundlage der redistributiven Bemühungen ist die ökonomische Absicherung gegenüber überraschenden Verminderungen des Realeinkommens. Die Unsicherheit bezüglich der zukünftigen Einkommensentwicklung bedingt, daß Individuen rationalerweise einen Teil ihres gegenwärtigen Einkommens freiwillig zur Verfügung stellen, um bei Eintritt des Versicherungsfalles ein bestimmtes Mindesteinkommen zu erhalten. In versicherungsökonomischem Kontext bildet das Motiv der Risikominderung den Gegenstand von Versicherungskontrakten, bei denen sich Leistung (Prämienzahlung) und Gegenleistung (Versicherungsschutz) gegenüberstehen. Als Begründung für die staatliche Durchführung dergestalt motivierter Bestrebungen wird die prinzipielle private Unversicherbarkeit bestimmter Risiken (Arbeitslosigkeit, Wirtschaftskrisen etc.) und die Problematik der "Adversen Selektion"1) bzw. des "Moral Hazard"2) angeführt. Die Argumentation beruht im wesentlichen darauf, daß sich bei der staatlichen Organisation der Transferaktivitäten die Möglichkeit eines effizienteren "Risk-Pooling" ${ }^{3)}$ bietet.

1) Private Versicherungen verweigern teilweise die Aufnahme von Individuen, die durch hohe Risikofaktoren gekennzeichnet sind. Vgl. Akerlof (1970), S. $492 \mathrm{ff}$.

2) Aufgrund des Versicherungsschutzes werden Individuen eigene Maßnahmen zur Reduzierung des Schadensrisikos vernachlässigen. Ein "moralisches Wagnis" (moral hazard) entsteht für die Anbieter von Versicherungen, die aufgrund unvollständiger Informationen ein fahrlässiges Verhalten der Versicherten nicht beobachten können. Vgl. Pauly (1968), S. $531 \mathrm{ff}$. bzw. (1974), S. $44 \mathrm{ff}$.

3) "The superiority of government in the insurance field rests on the existence of economies of scale in information collection and risk pooling which can be mopped up by the government but not by private firms ...." Brennan (1973), S. 52 . 
Betrachtet man auf der Grundlage des Versicherungsmotivs das Ausmaß der Sozialversicherung, ist allerdings zu vermuten, daß sich die staatliche Zwangsversicherung - im Vergleich zur privaten Versicherungslösung - nicht ausschließlich mit Effizienzargumenten rechtfertigen läßt.

Ein weiterer Erklärungsansatz beruht auf der Annahme interdependenter individueller Nutzenfunktionen ${ }^{1)}$. Die Unterscheidung zwischen negativen und positiven Nutzeninterdependenzen erlaubt die Berücksichtigung altruistischer und malevolenter Motive. Gehen beispielsweise das Einkommen des Begünstigten bzw. der Konsum bestimmter Güter ${ }^{2}$ ) als Argument in die Nutzenfunktion eines Wirtschaftssubjektes ein, kann im Fall positiver Nutzeninterdependenz ein benevolenter Transfergeber durch freiwillige Übertragung ökonomischer Ressourcen sein individuelles Nutzenniveau erhöhen ${ }^{3)}$.

Geht die "Verteilung" selbst als Argument in die individuelle Nutzenfunktion ein ${ }^{4)}$, wird ihr der Charakter eines rein öffentlichen Gutes zuteil. Aus der Kollektivguteigenschaft resultiert das bekannte Free-Rider-Phänomen der korrekten Präferenzoffenbarung im Hinblick auf die Bestimmung der jeweiligen Umverteilungsbeträge. Hieraus folgt die Rechtfertigung staatlicher Aktivitäten zur effizienten Bereitstellung des Gutes "Umverteilung".

1) Eine Zusammenfassung dieses Ansatzes findet sich bei Kubica $(1976)$, S. $36 \mathrm{ff}$.

2) Damit werden meritorische Erwägungen des Transfergebers einbezogen. Zur neueren Diskussion von Meritorisierungsaspekten vgl. Brennan/Lomasky (1983), S. $183 \mathrm{ff}$., Andel (1984), S. $630 \mathrm{ff}$. sowie Head (1988), S. $1 \mathrm{ff}$. bzw. auch Schmidt (1988), S. $383 \mathrm{ff}$.

3) Vgl. Hochman/Rodgers (1969), S. 542 ff.

4) Vgl. Thurow (1971), S. $328 \mathrm{f}$. 
Alle diese Ansätze sehen die Begründung für das redistributive Engagement des Staates in der Tatsache, daß kollektive Zwangsregelungen die hohen Transaktionskosten privater Kooperation vermeiden. Der Staat wird lediglich als Instrument zur effizienten Durchführung individuell gewünschter Redistributionshandlungen interpretiert, die auf freiwilliger Basis vorgenommen werden und zu Nutzensteigerungen bei den Beteiligten führen.

Obwohl diese überlegungen einen Teil der existierenden Redistributionsvorgänge rechtfertigen können ${ }^{1)}$, sind sie zur vollständigen Erklärung staatlicher Umverteilungstätigkeit nicht ausreichend, da Redistribution nicht nur die Konsequenz individueller Risikoverminderung oder ein Ergebnis karitativer Einstellungen ist, "sondern in gleicher Weise die Folge von Forderungen der Begünstigten und damit abhängig von politischen Strukturen" ${ }^{2}$ ).

Dieser Aspekt steht im Mittelpunkt des Rent-Seeking-Ansatzes, der die reale Verteilungsstruktur sowie die stattindenden Distributionsprozesse durch die Einflußnahme von Interessengruppen auf politische Entscheidungen zu erklären sucht. Aus dieser Perspektive wird der Staat zum "Produktionsfaktor" für Gruppen von Individuen mit Verteilungszielsetzungen, die durch staatliche Interventionen redistributive Interessen durchzusetzen wünschen. Das Ausmaß dieser Aktivitäten wird davon bestimmt, inwieweit die gegebenen politischen Institutionen Distributionsvorteile zugunsten einzelner Individuen oder gesellschaftlicher Gruppen zulassen ${ }^{3)}$.

1) Zur empirischen Bestimmumg der Höhe dieser freiwilligen Umverteilung auf der Grundlage des Versicherungsmotivs vgl. Zeckhauser (1974), S. $206 \mathrm{ff}$.

2) Vgl. Folkers $(1986 a)$, S. 21.

3) Vgl. Gäfgen (1983), S. 42. 
"Government is clearly the major source of monopoly power, and the theory of rent-seeking-behavior ... is likely to yield better insights by continuing to focus on this fact"1). Aus diesem Grund werden im folgenden alle Formen des Rent-Seeking im privaten Sektor nicht weiter betrachtet. Hierzu gehören beispielsweise die Versuche, durch wettbewerbsbehindernde Maßnahmen (z.B. Absprachen bzw. Kartellbildung) den marktlichen Allokationsmechanismus zu behindern oder ganz außer Kraft zu setzen. Die privaten Institutionen sind ebenfalls distributiv motiviert, besitzen allerdings den Nachteil, daß sie bei entsprechenden Gegenmaßnahmen potentieller Konkurrenten eher der Erosion unterliegen und entsprechend unwirksam werden. Im Gegensatz dazu sind die auf politischem Weg realisierten Regelungen daverhafter Natur und aufgrund der staatlichen Sanktionsmöglichkeiten für die langfristige Sicherung von "leistungsunabhängigen" Renten besonders geeignet.

Die Ausübung staatlicher Zwangsgewalt steht allerdings nicht mehr im Einklang mit den individuellen Präferenzen aller am Transferprozeß Beteiligten, weil bestimmten Individuen durch statliches Dekret die private Verfügungsgewalt über Teile des Realeinkommens entzogen oder im Vergleich zu anderen Wirtschaftssubjekten nicht gewährt wird. Da die Verfügungsgewalt über Ressourcen und ihre freiwillige Disponierbarkeit konstituierende Merkmale von Eigentumsrechten ${ }^{2}$ ) darstellen, kann auch von einer "Umverteilung von Property-Rights durch staatliches Dekret" ${ }^{3)}$ gesprochen werden.

1) DiLorenzo (1984), S. 195. In ähnlicher Formulierung auch $\operatorname{Orr}(1980)$, S. 224.

2) Zu einem allgemeinen Überblick vgl. Furubotn/Pejovich (1972), S. $1137 \mathrm{ff}$. Vgl. auch den Sammelband von Schüller (1983) mit den Beiträgen von Hesse (1983), S. 79 ff. sowie Röpke (1983), S. 111 ff.

3) Vgl. v. Weizsäcker (1984), S. 135. 
Während beim Profit-Seeking die Zuordnung von Eigentumsrechten an Ressourcen dem Einfluß der Wirtschaftssubjekte nicht unterliegt, wird beim Rent-Seeking explizit die Alternative einer Veränderung von Rechten durch politische Aktivitäten einbezogen. Eigentumsrechte beeinflussen die erwarteten Nutzenströme von Handlungsmöglichkeiten und damit den Wert der zu ihrer Realisierung benötigten Ressourcen. Jede Veränderung von Rechten bedeutet eine Umverteilung der mit einer bestimmten Aktion verbundenen Nutzenströme und damit auch eine Umverteilung ökonomischer Ressourcen. Eine erfolgreich durchgesetzte politische Regelung, die zu staatlich geschützten Renten führt, stellt damit eine Veränderung der Eigentumsrechte ${ }^{1}$ ) dar. Die Übertragung von Rechten durch politische Entscheidung ohne Kompensation der dadurch negativ betroffenen Wirtschaftssubjekte kann als politisch herbeigeführte Umverteilung interpretiert werden ${ }^{2}$ ).

Im Mittelpunkt spezieller Rent-Seeking-Vorhaben steht die Schaffung und Aufrechterhaltung spezifischer Verteilungsvorteile. Zum Zwecke dieser Untersuchung wird eine allgemeine Definition des Begriffs "Distributionsvorteil" gewählt, um die Vielzahl von staatlich ermöglichten Privilegien subsumieren zu können. Im folgenden werden mit diesem Begriff nicht nur alle Subventionen, Finanzhilfen, Transferzahlungen bzw.

Steuervergünstigungen und sonstige Unterstützungen des Staates bezeichnet, sondern auch jede vorteilhafte Veränderung von Steversätzen, Bemessungsgrundlagen etc. zugunsten spezifischer gesellschaftlicher Gruppen. Darüber hinaus sollen in dieser sehr weiten Begriffsbestimmung auch alle Verteilungsvorteile

1) Aus dieser Perspektive ist Rent-Seeking der Versuch "to define, reassign, modify, or attenuate property rights". Benson (1984), S. 398 .

2) "Entrepreneurs trade in property rights. Rent Seekers ... attempt to change by political means the established rights of others." Ricketts (1987), S. 462. 
erfaßt werden, die sich als Konsequenz der Gewährung staatlicher "Monopolprivilegien" in Form von begünstigenden Regulierungsmaßnahmen (Marktzutrittsschranken, Kontingentierungen etc.) ergeben und bestimmten Wirtschaftszweigen Zugang zu den daraus resultierenden Renten ermöglichen. Die Intensität der Rent-Seeking-Aktivitäten wird sich nach Art und Umfang der erwarteten Umverteilungsgewinne unterscheiden, die bei gegebenen Institutionen und politischen Bedingungen von der speziellen Form des Distributionsvorhabens abhängen. In gleicher Weise wird die Möglichkeit der erfolgreichen Durchsetzung "gruppenspezifischer Privilegien" durch Rent-Seeking entscheidend von der Organisationsfähigkeit der Gruppeninteressen bestimmt.

Im Rahmen dieser Untersuchung werden mit dem Begriff des RentSeeking alle Aktivitäten von Individuen und Gruppen bezeichnet, die durch Einflußnahme auf politische Entscheidungen eine Veränderung der sich aus dem Marktprozeß ergebenden Verteilungssituation anstreben. Es geht also nicht um die Analyse der Verteilung per se, sondern um den Prozeß der politisch herbeigeführten Änderung der Institutionen, die ein bestimmtes Verteilungsergebnis erzeugen. Da Distributionsgewinne für einzelne Gruppen mit entsprechenden Nachteilen für andere Individuen einhergehen, umfaßt diese allgemeine Definition nicht nur alle Bestrebungen zur Erlangung der Renten (Rent-Seeking i.e.S.) sondern auch alle Bemühungen zur Vermeidung von Rentenverlusten (Rent-Avoidance). Die Gesamtheit der Kosten der Organisation der Interessen, der Beeinflussungskosten im politischen Entscheidungsprozeß und im Informationsbereich (Lobbying) sollen als Rent-Seeking-Kosten bezeichnet werden. Die unterschiedlichen Interessenlagen und der dadurch induzierte Wettbewerb von Wirtschaftssubjekten bzw. Interessengruppen um individuell bzw. gruppenspezifisch begünstigende staatliche Aktivitäten sowie die resultierenden allokativen und distributiven Konsequenzen bilden den Untersuchungsgegenstand der Theorie des Rent-Seeking. 


\section{Die Entscheidung zwischen Profit-Seeking und Rent-Seeking}

Sind bestimmte Renten nicht durch Teilnahme am Markt sondern durch politische Einflußnahme zu erzielen, werden ökonomische Aktivitäten in diese Richtung entfaltet. Der Ressourceneinsatz zur Beeinflussung politischer Entscheidungen hängt dabei von den erwarteten Vorteilen im Vergleich zu den Kosten der Aktion ab. Bei der Darstellung der Entscheidung über die Teilnahme am Wettbewerb um marktliche und politische Renten kann auf das Rentenkonzept zurückgegriffen werden. Das Individuum hat neben der Verwendung der Ressourcen für Konsumzwecke die Wahl, seine verfügbaren Ressourcen entweder zu Profit-Seeking-Zwecken im Marktsektor ( $R^{M}$ ) oder für Rent-Seeking-Aktionen $\left(R^{S}\right)$ in Form politischer Aktivitäten einzusetzen ${ }^{1}$ ). Innerhalb dieses Spektrums individueller Ressourcendispositionen wird sich die Entscheidung je nach Art und Umfang sowie nach der Bedeutung der erzielbaren Rente für das einzelne Wirtschaftssubjekt unterscheiden. Dieser Zusammenhang wird in Abbildung 2 illustriert.

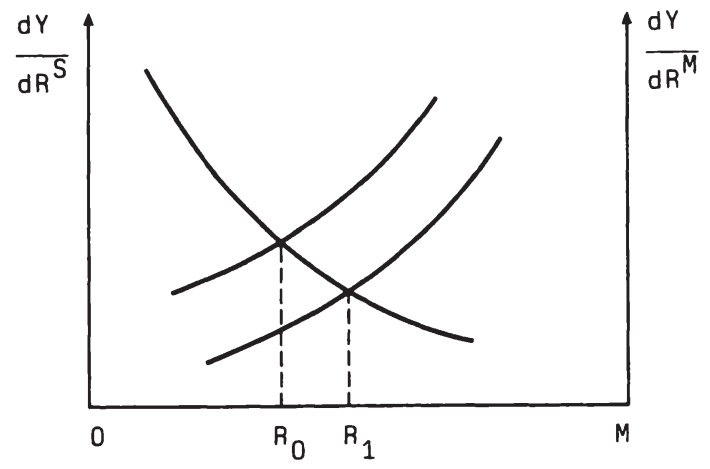

Abb. 2: Die Entscheidung zwischen Rent-Seeking und Profit-Seeking

1) Vgl. Lee/McKenzie (1987), S. 48 und Lee (1985a), S. $207 \mathrm{ff}$. 
Auf der Abszisse ist vom Ursprung 0 ausgehend der Ressourceneinsatz für Transferaktivitäten abgetragen, während von $M$ aus der Umfang der im Marktsektor investierten Ressourcen abgelesen werden kann. Die jeweiligen marginalen Erträge des Ressourceneinsatzes sind auf den entsprechenden Ordinaten zu erkennen ${ }^{1)}$. Bei konstanter Ressourcenaustattung OM wird solange in Rent-Seeking-Vorhaben investiert, bis der Grenzertrag dieser Aktivität den Opportunitätskosten in Form entgangener Erträge des marktlichen Ressourceneinsatzes entspricht. Die individuell optimale Aufteilung der Ressourcen auf beide Handlungsalternativen ergibt sich bei $R_{0}$, wobei $M R_{0}$ Einheiten für Profit-Seeking und $O R_{0}$ Einheiten für Rent-Seeking-Zwecke aufgewendet werden. Der Ressourceneinsatz zur Vermeidung von Umverteilungsverlusten kann in gleicher Weise dargestellt werden, wenn man den Ertrag der politischen Einflußnahme in Form von Ersparnissen aufgrund verhinderter Nachteile interpretiert. Aus dieser Darstellung der individuellen Entscheidungssituation zwischen Profit-Seeking und Rent-Seeking wird deutlich, daß jede staatliche Aktivität, die den Nettoertrag der im Marktsektor investierten Ressourcen schmälert, politische Einflußnahmeaktivitäten individuell vorteilhaft und damit ökonomisch rational macht ${ }^{2}$ ) (vgl. in Abbildung 2 die Ausdehnung von $R_{0}$ auf $R_{1}$ ). Ebenso müssen die Rent-SeekingAktivitäten anderer Wirtschaftssubjekte berücksichtigt werden. Allein die Tatsache, daß durch politische Einflußnahme Distributionsvorteile zu Lasten anderer Individuen oder gesellschaftlicher Gruppen möglich sind, führt dazu, daß die einzelnen Wirtschaftssubjekte zur Vermeidung von Umverteilungsverlusten zur Teilnahme am Distributionskonflikt gezwungen werden ${ }^{3)}$.

1) Zur individuellen Ressourcenaufteilung im gegebenen Zusammenhang vgl. Tullock (1967), S. 229 bzw. in allgemeiner Form bei Browning/Browning (1983), S. 173.

2) Vgl. dazu Orr (1980), S. 224 bzw. McKenzie (1981), S. 188.

3) Vgl. Tullock (1967), S. 229. 
4. Profit-Seeking und Rent-Seeking aus institutioneller Sicht

Zur Verdeutlichung des grundlegenden Unterschiedes des individuellen Strebens nach Renten im Rahmen des marktlichen bzw. des politischen Sektors einer ökonomie werden in übersicht 1 beide Ausprägungen des Wettbewerbs gegenübergestellt. Diese Form der Darstellung veranschaulicht die Notwendigkeit, "to examine and compare competitive processes that differ in ... the institutional setting under which the competitive process takes place"1).

Das charakteristische Merkmal der Institutionen beim ProfitSeeking liegt darin, daß sie für eine Vielzahl von Einzelentscheidungen der Wirtschaftssubjekte gelten. Die Existenz der marktlichen Institutionen ermöglicht, "für jedes zufällig herausgegriffene Mitglied der Gesellschaft die Chancen zu verbessern, die es hat, ein hohes Einkommen zu erzielen ... "2). Im Zentrum der Analyse steht die Frage nach den Ergebnissen des Wettbewerbs unter gegebenen Institutionen, an die sich Individuen mit ihren Entscheidungen anpassen. Die dabei anfallenden "natürlichen" Renten unterliegen vom Zeitpunkt ihrer Entstehung der marktlichen Konkurrenz. Der WettbewerbsprozeB führt zur Rentenerosion und gibt Anreize für unternehmerische Aktivitäten, die zur Steigerung des gesamtwirtschaftlichen Produktionsergebnisses beitragen. Die Konsequenzen marktlichen Wettbewerbs induzieren Rent-Seeking-Aktivitäten im Sinne einer Einflußnahme auf die Ausgestaltung der institutionellen Rahmenbedingungen, um Privilegien langfristig zu sichern bzw. marktliche Verluste abzuwenden ${ }^{3)}$.

1) Congleton (1980), S. 155 .

2) Hayek (1967), S. 28.

3) Vgl. Kirsch (1981), S. 255 ff. bzw. (1988), S. 117. 
Übersicht 1: Profit-Seeking versus Rent-Seeking

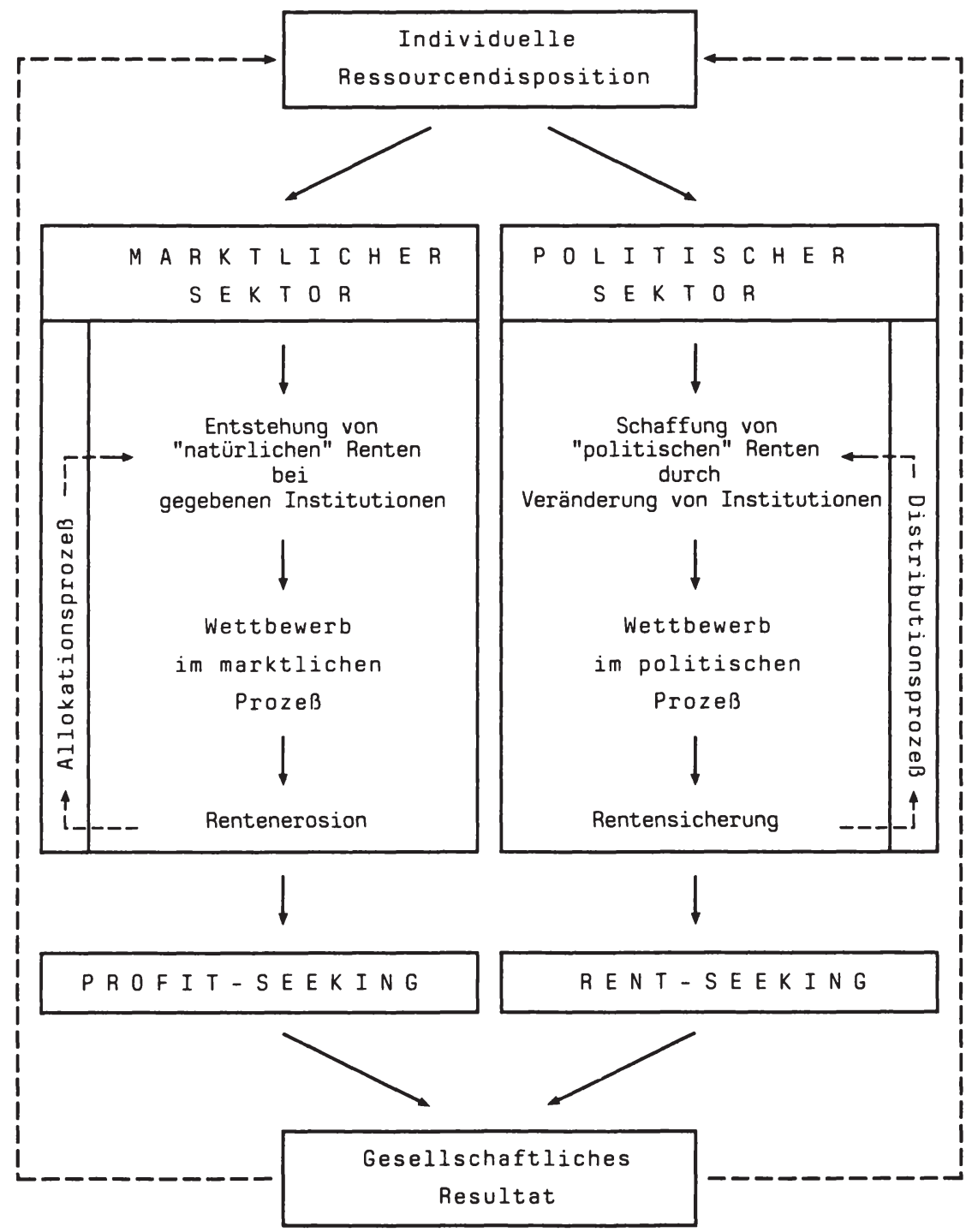


Ausgangspunkt der Rent-Seeking-Analyse ist die Feststellung, daß die Wirtschaftssubjekte "not only will ... pursue their selfinterest within the rules; they will also allocate resources toward changing the rules to their own benefit" ${ }^{1}$. Damit richtet sich das Interesse auf die Fragestellung, inwieweit die Institutionen durch individuelles Handeln beeinflußt werden können. Mit der Betonung des analytischen Unterschiedes zwischen dem Verhalten von Individuen unter gegebenen Institutionen und der Einflußnahme auf institutionelle staatliche Entscheidungen wird der ökonomische Ansatz in "vertikaler"2) Hinsicht ausgeweitet, indem die Problematik der Ausgestaltung der institutionellen Bedingungen individuellen Handelns untersucht wird ${ }^{3)}$. Die Forderungen nach bestimmten staatlichen Aktivitäten werden im Hinblick auf die erwarteten Effekte dieser Maßnahme bei laufenden ökonomischen Entscheidungen erhoben, d.h. die Konsequenzen staatlich determinierter Institutionen werden zum Motiv ihrer Einführung. Mit der Veränderung dieser Institutionen über den politischen Entscheidungsproze ${ }^{4}{ }^{\text {) }}$ wird eine systematische Verbesserung der Einkommenssituation einzelner Individuen oder Gruppen angestrebt.

1) Goldberg (1974), S. 461. In ähnlicher Weise konstatiert West (1978), S. 842, daß "people will use resources to profit themselves by actions outside the rules or directed to changing the rules".

2) Vgl. Vanberg (1986), S. 90.

3) Zu dieser grundlegenden Unterscheidung zwischen dem Verhalten unter gegebenen Regeln (Ebene der innerperiodischen bzw. laufenden Entscheidungen) und der Wahl zwischen alternativen Regelsystemen (Ebene der institutionellen Entscheidungen) sei auf Buchanan (1967) bzw. Brennan/ Buchanan (1980), S. 3 verwiesen.

4) "Bei einer Politisierung von Restriktionen erfolgt die Transformation von Zwängen unter den Eigengesetzlichkeiten des politischen Prozesses." Siebert (1985), S. 108. 
Die Zielsetzung beim Rent-Seeking liegt in der Durchsetzung von Ausnahmeregelungen der allgemeingültigen Institutionen bzw. in der begünstigenden Änderung fiskalischer Regeln. Als Begründung der Rent-Seeking-Vorhaben dient meist das Sozialstaatsprinzip ${ }^{1)}$, dessen inhaltliche offenheit die Durchsetzung immer never Distributionsvorhaben gestattet . Diese gruppenorientierten Privilegien eröffnen den Zugang zu leistungsunabhängigen "politischen" Renten zu Lasten anderer Wirtschaftssubjekte.

Als Konsequenz werden Bemühungen auf seiten der Belasteten induziert, die ihrerseits institutionelle Privilegien mit dem Hinweis auf Gleichbehandlung fordern. Alle Gruppen werden Ressourcen zur Durchsetzung ihrer gruppenspezifischen Interessen einsetzen. Institutionelle Veränderungen dieser Art führen zu einer "Rentenverfestigung", indem auf politischem Wege eine Rentenerosion vermieden wird ${ }^{2}$. Mit der Kumulation distributiv motivierter staatlicher Aktivitäten zugunsten spezifischer Gruppen werden Einkommen bzw. Einkommenserzielungsmöglichkeiten zunehmend durch politische Entscheidungen und entsprechend weniger durch marktliche Aktivitäten determiniert.

1) Der Gedanke des Sozialstaats ist im Grundgesetz der Bundesrepublik Deutschland in Art. 20 bzw. Art. 28 verankert.

2) Während das Profit-Seeking idealtypisch durch das Wirken der "invisible hand" gekennzeichnet ist, wird das RentSeeking auch durch die Wirkungsweise des "invisible foot" charakterisiert. Mit dieser Pointierung soll die Aufmerksamkeit auf die politischen Aktionen der Individuen gelenkt werden, die den Mechanismus der "invisible hand" zu behindern oder außer Kraft zu setzen suchen. "The invisible foot symbolizes the ... effects of political competition over redistribution." Brock/Magee (1984), S. 177. 
Besteht die Möglichkeit der - zur marktlichen Rentenrealisierung - komparativ vorteilhaften Durchsetzung von "politischen" Renten durch Einflußnahme auf den politischen Entscheidungsprozeß, so werden "potential profit-seeking entrepreneurs begin to turn away their attention from opportunities in the private sector, ...., toward opportunities for personal gain and private gain in the public or governmental sector of the economy"1). Der Wettbewerb um Renten im Wege des politischen Entscheidungsprozesses ist mit keiner produktiven Leistung verbunden, sondern der Ressourceneinsatz dient der Durchsetzung, Förderung bzw. Aufrechterhaltung von Distributionsvorteilen ${ }^{2)}$. Aufgrund dieser Zusammenhänge gewinnen Art, Umfang und Struktur der im politischen Prozeß festgelegten statlichen Institutionen zunehmend Einfluß auf die individuelle Ressourcendisposition ${ }^{3)}$. Die allokativen und distributiven Konsequenzen des Umverteilungskonflikts, die der Zugang politisch einflußreicher Gruppen zu den Möglichkeiten der Statsintervention hervorruft, stehen im Mittelpunkt der Theorie des Rent-Seeking ${ }^{4}$.

1) Buchanan (1978), S. 16 .

2) "Individuals and groups will seek to promote their own private distributional objectives, namely, increases in their own income shares, via government." Buchanan (1978), S. 9 .

3) Es ergeben sich Anreize für die Wirtschaftssubjekte "to change their behavior and use resources either to seek the transfer, avoid paying the cost or both". Formby/Miliner $(1987)$, S. 66 .

4) Die Theorie des Rent-Seeking "focus on the allocation of resources resulting from the competition over the control or use of government for private advantage". Samuels/ Mercuro (1984), S. 59. 


\section{Zusammenfassung}

Die Analyse der Funktionsweise marktlicher Institutionen bildet die Grundlage für die Beurteilung von staatlich determinierten Institutionen im Wohlfahrts-bzw. Interventionsstaat. Beim Profit-Seeking resultieren marktliche Renten als Konsequenz individuell rationalen Verhaltens unter den allgemeingültigen Institutionen des Marktsektors. Die Akzeptanz des marktlichen Verteilungsergebnisses hängt entscheidend davon $a b$, ob die institutionellen Bedingungen des Wettbewerbsprozesses den Konkurrenten gleiche Ausgangsvoraussetzungen gewähren. Das Postulat der Chancengleichheit findet seine Grenze in den unterschiedlichen angeborenen Fähigkeiten der einzelnen Individuen. Ergeben sich aus diesen Gründen soziale Nachteile, die eine Sicherung eines Mindesteinkommens zweifelhaft erscheinen lassen, soll der Staat durch unterstützende Maßnahmen die Verteilungssituation verändern können. Diese Form der staatlichen Aktivität ist allerdings strikt von den zahlreichen politischen Eingriffen in die marktlichen Institutionen bzw. in die Handlungsrechte anderer Wirtschaftssubjekte zu unterscheiden, bei denen die Funktionsweise des Marktsystems auf politischem Wege zur Absicherung einflußreicher gesellschaftlicher Gruppen außer Kraft gesetzt wird.

Die Theorie des Rent-Seeking basiert auf der Hypothese der "redistributiven Dominanz staatlicher Aktivitäten"1), wonach staatliche Maßnahmen vorwiegend unter dem Aspekt der - gegenüber der Alternative des marktlichen Profit-Seeking - komparativ vorteilhaften Durchsetzung von Sonderrechten zu erklären sind, mit der um politische Einflußnahme konkurrierende Individuen bzw. Interessengruppen das Ziel verfolgen, auf politischem Wege Renten zu erringen und damit Verteilungsvorteile abzusichern.

1) Windisch (1984), S. 589. 
Wenn bestimmte Eigentumsrechte unter dem politischen Druck von Interessengruppen durch staatliche Interventionen permanent verändert bzw. neu definiert werden können, wird es individuell ertragreich, Ressourcen zur Erlangung bzw. Aufrechterhaltung der damit verbundenen Verteilungsvorteile zu investieren. Materiell bestehen diese staatlichen Maßnahmen aus spezifischen administrativen Regulierungen (gezielter Protektionismus) und aus distributiv orientierten Regelungen im Bereich staatlicher Steuer- und Ausgabenpolitik (Steuervergünstigungen, Subventionen etc.), die einzelnen gesellschaftlichen Gruppen zugute kommen.

Gemäß der Rent-Seeking-Konzeption wirken insbesondere Interessengruppen auf die Durchführung begünstigender staatlicher Sonderregelungen seitens des States hin, um die Einkommen ihrer Mitglieder durch leistungsunabhängige Renten zu erhöhen. Je spezifischer die distributiven Gruppeninteressen sind, um so eher ist eine organisierte Einflußnahme auf den politischen Willensbildungsprozeß zu erwarten. Mit der Veränderung institutioneller Bedingungen werden meist "wohlerworbene" Ansprüche anderer gesellschaftlicher Gruppen tangiert, die ihrerseits Ressourcen zur Verhinderung der entsprechenden Regelung aufwenden werden. Aus der Interdependenz von organisierten Gruppeninteressen und politischem Entscheidungsverhalten kann sich ein kumulativer Prozeß ergeben, der die Entstehung weiterer staatlicher Maßnahmen mit vornehmlich distributivem Charakter induziert.

Der Prozeß der Änderung staatlicher Institutionen im Wege des politischen Entscheidungsprozesses sowie die Beurteilung der gesellschaftlichen Konsequenzen stehen im Zentrum der RentSeeking-Analyse. Das Resultat ist eine positive Theorie des Zustandekommens staatlicher Aktivitäten mit distributiver Zielsetzung im polit-ökonomischen Kontext. 


\section{DIE GRUNDSTRUKTUR DER RENT-SEEKING-ARGUMENTATION}

Als Ausgangspunkt der Untersuchung dient die Hypothese, daB Rent-Seeking-Aktivitäten entstehen, weil mit staatlichen Interventionen bestimmte Verteilungsvorteile realisierbar sind ${ }^{1)}$. Im Zentrum der herkömmlichen Analyse des Rent-Seeking, die auf den grundlegenden Beitrag "The Welfare Costs of Tariffs, Monopolies and Theft"2) von Gordon Tullock zurückgeht, steht die Frage nach den allokativen Konsequenzen des Ringens von Individuen und Interessengruppen um regulierungsinduzierte Renten. Dabei wird insbesondere die Problematik der Bemessung der von allen Interessenten in den Distributionswettbewerb investierten Ressourcen zur Realisisierung der staatlich abgesicherten Monopolprivilegien diskutiert.

Im Rahmen dieses Kapitels wird die Grundstruktur der RentSeeking-Argumentation aufgezeigt. Ausgehend vom bekannten Partialmodell des Rent-Seeking werden zunächst die Determinanten des individuellen und gesellschaftlichen Ressourceneinsatzes beim Akquisitionswettbewerb um staatlich ermöglichte Monopolprivilegien bestimmt. Auf dieser Grundlage wird als zentrales Resultat der Rent-Seeking-Analyse herausgearbeitet, daß die Allokationsverluste staatlicher Eingriffe in den Marktmechanismus in traditioneller Sicht unterschätzt werden. Anschließend werden einige der daraus resultierenden Schlußfolgerungen relativiert und bestimmte in der relevanten Literatur diskutierte Interpretationen bzw. Erweiterungen der grundlegenden Rent-Seeking-Konzeption problematisiert. Den Abschluß dieses Kapitels bildet eine Zusammenfassung der gewonnenen Resultate.

1) Vgl. Buchanan (1980a), S. 11.

2) Tullock (1967), S. 224 ff. 


\section{Das Grundmodell des Rent-Seeking}

Das grundlegende Konzept der Rent-Seeking-Theorie wurde in Verbindung mit der Analyse der Bestimmungsgründe regulierender staatlicher Eingriffe in den Marktmechanismus zugunsten einzelner Individuen bzw. bestimmter Wirtschaftszweige entwickelt, wobei vorwiegend die Frage nach den Ursachen der "arbitrary restrictions on market-freedom created through government auspices"1) untersucht wurde. Gegenstand der Analyse des Rent-Seeking sind die konkurrierenden Bemühungen der direkt von der staatlichen Maßnahme betroffenen Wirtschaftssubjekte im jeweiligen Markt. Das Streben nach einer "special position or monopoly power, is 'rent-seeking', and the 'rent' is the income derived"2). Die Zielsetzung des individuellen Handelns besteht in der Schaffung spezieller Schutzbereiche bzw. in der Verteidigung dieser Privilegien vor marktlicher Konkurrenz durch die Realisierung von Marktzutrittsbarrieren, Kontingentierungen, Zulassungsbeschränkungen und ähnlichen institutionellen Regelungen ${ }^{3)}$. Die Konkurrenz um statliche Regulierungseingriffe dient in erster Linie dem Schutz vor Wettbewerbseffekten bzw. der Kompensation unerwünschter marktlicher substitutionswirkungen ${ }^{4}$ ).

1) Buchanan (1980b), S. 183.

2) Tullock (1980a), S. 17 .

3) Rent-Seeking wird in diesem Zusammenhang gleichbedeutend mit der Bezeichnung "Tariff-Seeking", "Premium-Seeking" bzw. "Monopoly-Seeking" verwendet. Vgl. Bhagwati (1982a), S. 992. In allgemeiner Form findet sich auch die Bezeichnung "favor seeking". Vgl. Wellisz/Findlay (1984), S. 148.

4) Vgl. Streit (1986a), S. 99. 
Im weiteren dient der Begriff "Monopolprivileg" als Oberbegriff für die Vielzahl staatlich gewährter Sonderrechte zugunsten einzelner Wirtschaftssubjekte. Die damit verbundenen politischen Renten ergeben sich als Konsequenz der umfangreichen "restrictions on other potential competitors, restrictions that are artificial or contrived in not being naturally inevitable"1). Gelingt eine erfolgreiche Durchsetzung einer begünstigenden Regelung, wird der Zugang zu abgesicherten Distributionsvorteilen ermöglicht.

Die Struktur der Rent-Seeking-Argumentation ${ }^{2}$ ) kann in Analogie zur Bestimmung der allokativen Effekte einer Monopolbildung demonstriert werden. Mit der Einführung einer protektionistischen Regelung ist eine Abweichung von der Preis-MengenKombination $\left(p_{0}, x_{0}\right)$ des Konkurrenzgleichgewichts verbunden (Abbildung 3). Erfolgt z.B. eine Marktzutrittsbeschränkung, ist das Angebot auf $x_{1}$ festgelegt, und es resultiert eine Preiserhöhung auf $p_{1}$ im neven Gleichgewicht. Als Konsequenz dieser Maßnahme ergibt sich ein Verlust an Konsumentenrente in Höhe von $p_{1} A C p_{0}$.

1) Alchian (1987), S. 143. Die zahlreichen staatlichen Restriktionen reichen von Zulassungsbeschränkungen für einzelne Berufe (z.B. Ärzte, Rechtsanwälte, Taxifahrer etc.) über Regulierungen im Straßengüterfernverkehr, im Bereich des Handwerks bis hin zu vielfältigen Einzelregelungen (Nachtbackverbot, Ladenschlußgesetze). "It should be immediately emphasized that this does not imply nor it is to be inferred that all such restrictions are demonstrably undesirable. Nevertheless, the increased wealth potential is a 'monopoly rent'." Alchian (1987), S. 143.

2) Vgl. Tullock (1967), S. 225 bzw. (1984b), S. 227 aber auch Tollison (1982), S. 580 und (1987), S. 146 sowie Tullock $(1988$ a), S. 52 . 


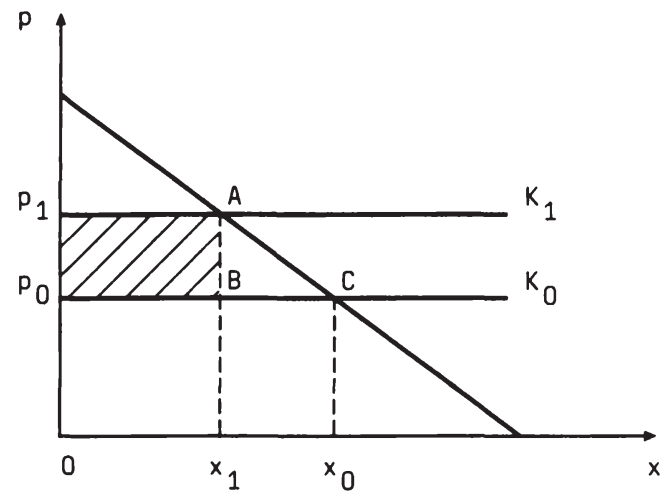

Abb. 3: Die sozialen Kosten des Wettbewerbs um Monopolprivilegien

Während nach traditioneller Ansicht ${ }^{1)}$ die entstehenden Rentengewinne in Form der Fläche $p_{1} A B p_{0}$ lediglich als Transfer von Konsument zu Produzent interpretiert wurden, stellt die Fläche $A B C$ den allokativen Verlust aufgrund der Monopolbildung dar. Diese Mehrbelastung bezeichnet den Verlust an Rente, der einer Ökonomie als Konsequenz der Intervention in den Marktmechanismus endgültig verloren geht ${ }^{2}$. Aus der Rent-Seeking-Perspektive wird die Aufmerksamkeit auf die Tatsache gelenkt, daß die potentielle Existenz von Monopolrechten ${ }^{3}$ ) zu politischen Aktivitäten führen wird, um das Privileg durch statliche Entscheidung zugesprochen zu bekommen.

1) Diese Interpretation beruht auf der Annahme, daß "the monopoly is created in a costless manner, perhaps by an act of God, whereas in fact real resources are used to create monopolies". Tullock (1987a), S. 147.

2) Vgl. Harberger (1954), S. $77 \mathrm{ff}$.

3) Vgl. Tollison (1982), S. $581 \mathrm{ff}$. 
Die erfolgreich realisierten Renten müssen nicht unbedingt beim Produzenten verbleiben, sondern können auch in Form von - gegenüber der Konkurrenzsituation - erhöhten Faktorentlohnungen oder durch Abweichung von der Minimalkostenkombination an andere Individuen weiterverteilt werden ${ }^{1)}$ (vgl. die Verschiebung von $K_{0}$ auf $K_{1}$ in Abbildung 3 ). Bezieht man die Möglichkeit der Weiterverteilung der mit Interventionen in den Marktmechanismus verbundenen Rentengewinne ein, werden alle Versuche zur Durchsetzung des Monopolprivilegs die Unterstützung der Individuen finden, die an diesen Renten partizipieren wollen ${ }^{2}$. Beispielsweise haben alle involvierten Produktionsfaktoren ein Interesse an der Aufrechterhaltung der auf politischem Wege durchgesetzten Privilegierung und werden in dieser Konstellation gemeinsam agieren. Vor allem Individuen aus Sektoren der Wirtschaft, die sich aufgrund der marktlichen Entwicklung einer unsicheren Zukunft gegenübersehen, werden versuchen, durch vorteilhafte staatliche Regelungen Verluste zu verhindern. "Die Begünstigten - nicht selten Unternehmer und Arbeitnehmer gemeinsam - sehen es als lohnender an, sich um staatlichen Schutz gegen Konkurrenz zu bemühen, als durch eigene Anstrengung Wege aus der Misere zu finden, und selbst florierende Unternehmen verlieren ... die Scheu, sich öffentlicher Hilfen zu bedienen" ${ }^{3}$ ?

1) "The best of all monopoly profits is a quiet life." Hicks (1935), S. 8. Zur Problematik der X-Ineffizienz vgl. z.B. Leibenstein (1966) oder Stigler (1976). Im gegebenen Zusammenhang vgl. auch Formby/Keeler/Thistle (1988), S. 115 ff.

2) Zur empirischen Analyse des Verhaltens von Gewerkschaften unter Rent-Seeking-Aspekten vgl. Addison/Genosko/Schnabel (1989), S. 102 ff.

3) Sachverständigenrat zur Begutachtung der gesamtwirtschaftlichen Entwicklung, Jahresgutachten 1988/89, S. 140. 
Die Möglichkeit der Realisierung begünstigender Regelungen in Form von staatlichen Eingriffen in die individuelle Handlungsund Vertragsfreiheit stellt eine Begründung für das umfangreiche System statlicher Regulierungen dar, das durch vielfältige Ausnahmeregelungen und Vergünstigungen zugunsten einzelner gesellschaftlicher Gruppen gekennzeichnet ist ${ }^{1}$.

Die staatlich legitimierten Regelungen bilden die institutionellen Voraussetzungen ${ }^{2}$ ), die daverhafte Rentengewinne garantieren, indem sie den marktlichen Erosionsprozeß außer Kraft setzen. Darüber hinaus beschränken sie den individuellen Handlungsspielraum, verändern das marktwirtschaftliche Verteilungsergebnis und induzieren Anreize, politische Instanzen ${ }^{3}$ ) für eine weitere begünstigende Ausgestaltung von Gesetzen zu gewinnen. Gleichzeitig sind politische Interventionen in Form regulierender Normen und Vorschriften geeignet, um die angestrebte Distributionszielsetzung im politischen Prozeß durchzusetzen, wenn sie mit der Wahrung des öffentlichen Interesses gerechtfertigt werden ${ }^{4}$ ).

1) Die Theorie des Rent-Seeking relativiert die Vorstellung der "ruinösen Konkurrenz" als Begründung von staatlichen Markteingriffen. Obwohl dieser Erklärungsansatz sicher für bestimmte Bereiche zutrifft, ist aus der Rent-SeekingPerspektive zu vermuten, daß "das Argument der ruinösen Konkurrenz weit überdehnt wird, um damit interessierte Gruppen vor Wettbewerb zu schützen". v. Weizsäcker (1982), S. 330 .

2) Als Beispiel sei auf normierte Arbeitszeitregelungen, Überstundenverbote bzw. Weitere Beschäftigungseinschränkungen verwiesen, die den bereits beschäftigten Ressourcenanbietern gesicherte Renteneinkommen ermöglichen. Vgl. dazu Meyer (1987), S. $885 \mathrm{ff}$. sowie (1988), S. $21 \mathrm{ff}$.

3) Vgl. Mounts Jr./Sowell/Lindley (1985), S. 87 ff.

4) Vgl. Pasour Jr. (1985), S. $527 \mathrm{ff}$. 


\section{Das Modell des kompetitiven Rent-Seeking}

Die Vorstellung eines Wettbewerbs um eine mit regulierenden Eingriffen verbundene staatlich gesicherte Monopolrente rückt die Frage nach dem Umfang der Ressourcen, den die Interessenten zur Erlangung des Monopolprivilegs aufwenden, in den Mittelpunkt der Rent-Seeking-Analyse. Mit dem Begriff des "kompetitiven" Rent-Seeking soll zum Ausdruck gebracht werden, daß "members of some group compete with each other in order to acquire some sort of monopoly position or other special treatment for the winner of the competition"1). Im folgenden werden wichtige Bestimmungsfaktoren des Umfangs der im Akquisitionswettbewerb eingesetzten volkswirtschaftlichen Ressourcen näher untersucht.

\subsection{Der Wettbewerb um Monopolprivilegien}

Ausgangspunkt der überlegungen sei eine exogen gegebene und in ihrem Ausmaß bekannte Monopolrente Tr, um die sich $n$ Individuen (z.B. Produzenten eines Wirtschaftszweiges) bemühen ${ }^{2}$ ). Aufgrund der Unteilbarkeit des Monopolrechts kann nur eines der Wirtschaftssubjekte das Privileg zugesprochen bekommen, während alle anderen Teilnehmer am Wettbewerb um den Transferbetrag unterliegen. Diese Konstellation entspricht im wesentlichen der Teilnahme an einer Lotterie, bei der Individuen darüber entscheiden müssen, inwieweit sie weitere Lose erwerben, um den ausgesetzten Preis zu gewinnen.

1) Varian (1983), S. 20.

2) Die Analyse des Wettbewerbs um die Erteilung von Importlizenzen bildet den Gegenstand der Untersuchung von Krueger (1974). Die Konkurrenz um staatlich gesicherte Monopolrenten untersucht Posner (1975). 
Zur Bestimmung des individuellen Ressourceneinsatzes wird auf einen spieltheoretischen Lösungsansatz zurückgegriffen ${ }^{1}$ ). Die Wahrscheinlichkeit für die erfolgreiche Realisierung des Monopolrechts durch ein Individuum $i$ wird gemäß

( III.1)

$$
\pi_{i}=f_{i}(R) \quad \text { für } i=1,2, \ldots, n
$$

ermittelt, wobei

(III.2)

$$
R=\left(R_{1}, R_{2}, \ldots, R_{n}\right)
$$

den Vektor der von allen Interessenten in den Wettbewerbsprozeß investierten Ressourcen darstellt ${ }^{2}$.

Dabei soll entsprechend

(III.3)

$$
\frac{d f_{i}}{d R_{i}}>0 \quad b z w . \quad \frac{d f_{i}}{d R_{j}}<0 \quad \text { für } i \neq j
$$

eine positive Grenzproduktivität des eigenen Ressourceneinsatzes $\left(R_{i}\right)$ bzw. ein negativer Einfluß marginaler Rent-SeekingInvestitionen der Konkurrenz $\left(R_{j}\right)$ in bezug auf die Veränderung der individuellen Erfolgswahrscheinlichkeit des betrachteten Wirtschaftssubjektes vorliegen ${ }^{3}$ ).

1) Vgl. dazu insbesondere Tullock (1980b), S. $101 \mathrm{ff}$. bzw. auch $(1988 \mathrm{~b})$, S. $91 \mathrm{ff}$.

2) Vgl. Hillman/Katz (1984), S. 106 bzw. Rice/Ulen (1981), S. 60 .

3) Vgl. Wickström (1987), S. 41. 
Spezifiziert man (III.1) in Form von

( III.4)

$$
\pi_{i}=\frac{R_{i}^{\alpha}}{\sum_{j=1}^{n} R_{j}^{\alpha}}
$$

als den Anteil des Ressourceneinsatzes von $i$ an den gesamten in den Umverteilungsprozeß eingebrachten Ressourcen ${ }^{1)}$, bringt der Parameter

$$
\alpha=\frac{d \pi_{i}}{\pi_{i}}: \frac{d R_{i}}{R_{i}}
$$

die Elastizität des Ressourceneinsatzes für Distributionszwecke in bezug auf die individuelle Gewinnwahrscheinlichkeit zum Ausdruck ${ }^{2}$ ). Dieser Parameter symbolisiert die Kosten der Ausübung politischen Einflusses, d.h. eine Erhöhung (Verminderung) des Wertes von $\alpha$ bedeutet eine Reduktion (Steigerung) der Grenzkosten des Rent-Seeking-Einsatzes. Das Reaktionsverhalten des betrachteten Individuums im Hinblick auf Veränderungen der Rent-Seeking-Investitionen der Mitkonkurrenten werde durch die Cournot-Nash-Annahme wiedergegeben ${ }^{3)}$.

1) Zur Verwendung dieser Funktion vgl. Tullock (1980b), S. 101 bzw. Appelbaum/Katz (1986a, 1986b) aber auch Corcoran (1984), Corcoran/Karels (1985) bzw. Higgins/Shughart II/ Tollison (1985).

2) Die Bedeutung des Parameters a betonen insbesondere Tullock (1980b), S. 101 aber auch Higgins/Shughart II/Tollison $(1985)$, S. 247.

3) Zur Diskussion anderer Verhaltensannahmen sei auf Michaels (1989), S. $31 \mathrm{ff}$. und Smith/Weber (1989), S. $153 \mathrm{ff}$. verwiesen. 
Nach dieser Verhaltenshypothese ${ }^{1)}$ wird eine konjekturale Variation der Form

( III.6)

$$
\frac{d R_{i}^{\alpha}}{d R_{j}^{\alpha}}=0
$$

unterstellt, d.h. es erfolgen keine Reaktionen im Hinblick auf den eigenen Ressourceneinsatz bei Veränderungen der Investitionen anderer Interessenten.

Unter diesen Annahmen läßt sich für alle am Wettbewerbsprozeß teilnehmenden Individuen ein Maximierungsproblem gemäß

$$
E\left(G_{i}\right)=\pi_{i}\left(\operatorname{Tr}-R_{i}^{\alpha}\right)+\left(1-\pi_{i}\right)\left(-R_{i}^{\alpha}\right) \rightarrow \max !
$$

formulieren, wobei $E\left(G_{i}\right)$ den Erwartungswert des Gewinns bezeichnet.

Die Bedingung erster Ordnung läßt sich in Form von

(III.8)

$$
\frac{d E\left(G_{i}\right)}{d R_{i}}=\frac{d \pi}{d R_{i}} \cdot T r-1=0
$$

ermitteln, d.h. der marginale Ressourceneinsatz muß der dadurch induzierten Steigerung der Wahrscheinlichkeit einer erfolgreichen Realisierung des Monopolprivilegs entsprechen ${ }^{2}$ ).

1) Die Cournot-Nash-Reaktionshypothese ist für solche Entscheidungssituationen nicht untypisch. Allerdings impliziert sie eine Art des Nicht-Lernens, da die Wirtschaftssubjekte bei Veränderungen des Ressourceneinsatzes anderer Individuen keine Anpassungsreaktionen vornehmen.

2) Vgl. Tullock (1987a), S. 147. 
Der Differentialquotient in (III.8) stellt sich in Form von

( III.9)

$$
\frac{d \pi_{i}}{d R_{i}}=\frac{\alpha R_{i}^{\alpha-1}\left(\sum_{j=1}^{n} R_{j}^{\alpha}\right)-\alpha R_{i}^{2 \alpha-1}}{\left(\sum_{j=1}^{n} R_{j}^{\alpha}\right)^{2}}
$$

dar. Im weiteren wird davon ausgegangen, daß bei Existenz einer optimalen Strategie alle Teilnehmer am Wettbewerb um das Monopolrecht in der Lage sind, diese auch zu identifizieren. Als Konsequenz dieser Annahme ${ }^{1)}$ resultiert gemäß

( III.10)

$$
R_{i}=R_{j}
$$

ein symmetrischer Ressourceneinsatz aller Interessenten und damit eine identische Gewinnwahrscheinlichkeit ${ }^{2}$. Unter Verwendung dieser Beziehung kann (III.9) zu

( III.11)

$$
\frac{d \pi}{d R}=\frac{\alpha(n-1)}{n^{2} R}
$$

vereinfacht werden, woraus nach Substitution in (III.8)

1) Tullock betont diesen Aspekt explizit, indem er konstatiert, "if there is a correct solution for individual strategy, then each player will assume that the other parties can also figure out what that correct solution is". Tullock (1980b), S. 99. Vgl. auch Appelbaum/Katz (1986a), S. 177 bzw. VanLong/Vousden (1987), S. 974.

2) Vgl. in modifizierter Form Allard (1988), S. 3 ff. 
unmittelbar

(III.12) $\quad \frac{d E(G)}{d R}=\frac{\alpha(n-1)}{n^{2} R}-1=0$

folgt.

Unter Berücksichtigung von (III.8) kann aus dieser Beziehung die Aussage abgeleitet werden, daB nur dann Ressourcen zur Erlangung des Monopolprivilegs aufgewendet werden, wenn tatsächlich Mitbewerber vorhanden sind. Ist die politische Rente aufgrund fehlender Konkurrenten $(n=1)$ mit Sicherheit zu erwarten, wird ein entsprechender Ressourceneinsatz unterbleiben ${ }^{1)}$.

Als hinreichende Bedingung muß

(III.13) $\quad \frac{d^{2} \pi}{d R^{2}}=\frac{[(n-2) \alpha-n] \alpha(n-1)}{n^{3} R^{2}}<0$

gelten, die nur für

(III.14) $\quad(n-2) \alpha-n<0$

und damit

( III.15)

$\alpha<\frac{n}{n-2}$

erfüllt ist.

1) Vgl. Tullock (1980b), S. 103 bzw. Corcoran (1984), S. 91. 
Da der Term auf der rechten Seite von (III.15) immer größer als eins ist, wird die Stabilitätsbedingung ${ }^{1)}$ in Form von $\alpha \leqq 1$

bestimmt. Der optimale Ressourceneinsatz $R^{\star}$ für den einzelnen Teilnehmer am Akquisitionswettbewerb wird als

( I I . 17)

$$
R^{\star}=\frac{(n-1)}{n^{2}} \cdot \alpha \operatorname{Tr}
$$

ermittelt ${ }^{2}$ ).

Offensichtlich steigt $R^{*}$ mit der Höhe des zur Disposition stehenden Transferbetrages Tr an. Ebenso resultiert bei einer Verminderung der Grenzkosten, die durch einen erhöhten Wert des Parameters $\alpha$ zum Ausdruck kommt, ein Anstieg der für distributive Zwecke aufgewendeten Ressourcen des Individuums. Dagegen ist mit einem Anstieg der Anzahl der Konkurrenten im Wettbewerbsprozeß gemäß

$$
\text { (III.18) } \quad \lim _{n \rightarrow \infty} R^{*}=0
$$

eine Reduktion des individuellen Ressourceneinsatzes verbunden ${ }^{3)}$.

1) Vgl. dazu Tullock (1980b), S. $105 \mathrm{ff}$.

2) Vgl. Rice/Ulen (1981), S. 61.

3) Vgl. Tullock (1988b), S. $92 \mathrm{f}$. 
Die Ermittlung der von allen Interessenten bei ihren Bemühungen zur Erlangung der regulierungsinduzierten Monopolrente investierten Ressourcen $W$ läßt sich in Form von

$$
W=n R^{\star}=\frac{n-1}{n} \cdot \alpha T r
$$

darstellen. Danach steigt der gesamte Ressourceneinsatz mit dem zur Disposition stehenden Transferbetrag sowie dem Parameter $\alpha$ an. Der Grenzwert des Ressourceneinsatzes bei zunehmender Anzahl der Konkurrenten ergibt sich als

$$
\lim _{n \rightarrow \infty} w=\alpha \operatorname{Tr}
$$

wobei ein direkter Zusammenhang zwischen dem gesamten Ressourceneinsatz und der Höhe der politischen Rente hergestellt wird.

Zur Illustration der Ergebnisse dienen die Tabellen 1 bzw. 2, die den individuellen bzw. gesellschaftlichen Ressourceneinsatz für Rent-Seeking beim Wettbewerb um eine exogen gegebene Monopolrente in Höhe von 100 Geldeinheiten wiedergeben. Dabei wurden die entsprechenden Werte für variierende Teilnehmerzahlen $(n)$ bzw. Unterschiedliche Kostenstrukturen $(\alpha)$ auf der Grundlage der Beziehungen (III.17) bzw. (III.19) ermittelt ${ }^{1}$ ).

1) Zur Diskussion um die Berechnung des Ressourceneinsatzes auf der Grundlage dieses Modells sei auf die Beiträge von Tullock (1980b), S. $102 \mathrm{ff}$., Corcoran (1984), S. $89 \mathrm{ff}$., Tullock (1984a), S. 95 ff. bzw. Corcoran/Karels (1985), S. $227 \mathrm{ff.}$. Higgins/Shughart II/Tollison (1985), S. $247 \mathrm{ff}$. und Tullock (1985), S. $259 \mathrm{ff}$. sowie (1988b), S. $91 \mathrm{ff}$. verwiesen. In dynamischem Kontext vgl. Maußner (1986). 
Tab. 1: Individueller Ressourceneinsatz beim Wettbewerb um eine Monopolrente (in Höhe von $100 \mathrm{GE}$ ) ${ }^{1}$ )

\begin{tabular}{|r|rrrrr|}
\hline$\alpha$ & 2 & 4 & 10 & 15 & $\infty$ \\
\hline 0,5 & 12,50 & 9,38 & 4,50 & 3,11 & 0 \\
1,0 & 25,00 & 18,75 & 9,00 & 6,22 & 0 \\
2,0 & 50,00 & 37,50 & 18,00 & 12,44 & 0 \\
3,0 & 75,00 & 56,25 & 27,00 & 18,67 & 0 \\
8,0 & 200,00 & 150,00 & 72,00 & 49,78 & 0 \\
\hline
\end{tabular}

Tab. 2: Gesellschaftlicher Ressourceneinsatz beim Wettbewerb um eine Monopolrente (in Höhe von $100 \mathrm{GE}$ )

\begin{tabular}{|r|rrrrr|}
\hline$\alpha$ & 2 & 4 & 10 & 15 & $\infty$ \\
\hline 0,5 & 25,00 & 37,50 & 45,00 & 46,67 & 50,00 \\
1,0 & 50,00 & 75,00 & 90,00 & 93,33 & 100,00 \\
2,0 & 100,00 & 150,00 & 180,00 & 186,00 & 200,00 \\
3,0 & 150,00 & 225,00 & 270,00 & 280,00 & 300,00 \\
8,0 & 400,00 & 600,00 & 720,00 & 746,00 & 800,00 \\
\hline
\end{tabular}

1) In Anlehnung an Tullock (1980b), S. 102 bzw. (1988b), S. $91 \mathrm{ff}$. 
Mit steigender Anzahl der Interessenten ist ceteris paribus eine Abnahme der individuellen Rent-Seeking-Investitionen verbunden, während der soziale Ressourceneinsatz steigt. Als Resultat der Analyse des kompetitiven Rent-Seeking ist festzuhalten, daß die von allen Interessenten in den Wettbewerb um das Sonderrecht investierten Ressourcen nur dann genau dem Wert des zur Disposition stehenden Transferbetrages entsprechen, wenn konstante Ertragszuwächse des Ressourceneinsatzes $(\alpha=1)$ in bezug auf die Erhöhung der individuellen Wahrscheinlichkeit zur erfolgreichen Realisierung des Monopolprivilegs vorliegen. "In a way, what is happening here is that a monopoly profit is being competed away"1).

Eine Gleichgewichtslösung resultiert in einer transparenten Wettbewerbssituation und bei linearer oder fallender Funktion der Erfolgswahrscheinlichkeit $(\alpha \leqq 1)$. Allerdings kann in Abhängigkeit vom Parameter $\alpha$ der soziale Ressourceneinsatz den Wert des Monopolrechts auch über-bzw. unterschreiten ${ }^{2}$. So wird beispielsweise für eine quadratische Wahrscheinlichkeitsfunktion $(\alpha=2)$ bereits das Doppelte des zur Disposition stehenden Transferbetrages investiert, wobei aufgrund der Verletzung der Stabilitätsbedingung keine Gleichgewichtslösung existiert. Im Fall unterschiedlicher Ausgangssituationen der Teilnehmer am Distributionswettbewerb ergeben sich für den derzeitigen Besitzer des Privilegs gewisse "Startvorteile", so daß bei periodischer Vergabe des Rechtes der Ressourceneinsatz den Wert der Monopolrente unterschreitet ${ }^{3}$.

1) Tullock (1980b), S. 102 f. bzw. Hillman/Samet (1987a), S. 63 und Tullock (1987a), S. $147 \mathrm{f}$. bzw. (1987b), S. $83 \mathrm{f}$. oder McKenzie (1988), S. 87.

2) Eine Darstellung verschiedener Modifikationen des Grundmodells findet sich bei Hillman/Samet (1987a, 1987b).

3) Vgl. Rogerson (1982), S. 394 ff. bzw. Rice/ulen (1981), S. $62 \mathrm{ff}$. aber auch Tollison (1989), S. $97 \mathrm{f}$. 


\subsection{Der Wettbewerb um Monopolprivilegien unter Berück- sichtigung von Unsicherheit}

Zur Erweiterung der bisherigen Überlegungen soll die Problematik unterschiedlicher Risikoeinstellungen ${ }^{1)}$ der Konkurrenten beim Wettbewerb um Monopolprivilegien untersucht werden. Dabei wird der Frage nachgegangen, inwieweit das in der herkömmlichen Analyse dominierende Ergebnis einer "vollständigen Rentenabschöpfung", d.h. die Bemessung des gesellschaftlichen Ressourceneinsatzes in Höhe des zur Disposition stehenden Transferbetrages, aufrechterhalten werden kann.

Um den Aspekt von Unsicherheit der Teilnehmer am Akquisitionswettbewerb isoliert betrachten zu können, werden auf der Grundlage der gemäß (III.4) spezifizierten Funktion der individuellen Erfolgswahrscheinlichkeit zur Erlangung der begünstigenden Monopolstellung konstante Ertragsraten bei zunehmendem Ressourceneinsatz in bezug auf die Steigerung der Realisierungschancen der Monopolrente $(\alpha=1)$ unterstellt. Weiterhin wird von identischen Nutzenfunktionen der Interessenten am Monopolprivileg ausgegangen, die den Axiomen von von Neumann-Morgenstern ${ }^{2}$ ) genügen sollen.

1) "Uncertainty is intrinsic in a competitive quest to attain a biddable monopoly rent, since the rent by its nature accrues indivisibly to the one rent-seeker, who is to be ultimate monopolist." Hillman/Katz (1984), S. 104. Zur Berücksichtigung von Unsicherheit bzw. unterschiedlicher Risikoneigungen der Teilnehmer am WettbewerbsprozeB vgl. Jadlow (1985), S. $73 \mathrm{ff}$. und VanLong/Vousden (1987), S. $971 \mathrm{ff}$. sowie Fabella (1989), S. $199 \mathrm{ff}$.

2) Vgl. v. Neumann/Morgenstern (1973), S. 26 ff. bzw. Hey (1979), S. 27 ff. oder Menges (1974), S. 53 ff. Insbesondere wird neben bestimmten Monotonieeigenschaften die zweimalige Differenzierbarkeit der Nutzenfunktion vorausgesetzt. 
Als Argument einer solchen Nutzenfunktion sei ein bestimmtes Ausgangsvermögen $A$ enthalten, das bei Nichtteilnahme am RentSeeking-Wettbewerb mit Sicherheit verbleibt. Damit läßt sich die Zielfunktion eines repräsentativen Interessenten als

$$
\begin{array}{r}
E\left(U_{i}\right)=\pi_{i} U_{i}\left(A+T r-R_{i}\right)+\left(1-\pi_{i}\right)\left(A-R_{i}\right) U_{i} \\
\\
\rightarrow \max !
\end{array}
$$

darstellen, wobei $E\left(U_{i}\right)$ den Erwartungsnutzen symbolisiert. Die notwendige Bedingung wird unter Verwendung der Symmetrieannahme (III.10) als

( III.22)

$$
\begin{aligned}
\frac{d E(U)}{d R} & =\frac{1}{n}\left[U^{\prime}(A-R)-U^{\prime}(A+T r-R)\right]-U^{\prime}(A-R) \\
& +\frac{n-1}{n^{2} R}[U(A+T r-R)-U(A-R)]=0
\end{aligned}
$$

abgeleitet, woraus bei Berücksichtigung der Beziehungen (III.18) und (III.19)

(III.23)

$$
\lim _{n \rightarrow \infty} \frac{U(A+\operatorname{Tr})-U(A)}{W}-U^{\prime}(A)=0
$$

und damit

(III.24)

$$
\lim _{n \rightarrow \infty} U(A+\operatorname{Tr})=U^{\prime}(A) W+U(A)
$$

resultiert. 
Die Approximation dieses Ausdrucks erfolgt mit Hilfe der Taylor'schen Reihenentwicklung ${ }^{1)}$, so daß sich

$$
\begin{aligned}
U(A+T r) & =U(A)+U^{\prime}(A) T r+\frac{U^{\prime \prime}(A)}{2} \cdot \operatorname{Tr}^{2} \\
& +\frac{U^{\prime \prime \prime}(A)}{6} \cdot \operatorname{Tr}^{3}
\end{aligned}
$$

ergibt. Durch Substitution in (III.24) wird über

(III.26)

$$
\lim _{n \rightarrow \infty} U(A)+U^{\prime}(A) \operatorname{Tr}+\frac{U^{\prime \prime}(A)}{2} \cdot \operatorname{Tr}^{2}+\frac{U^{\prime \prime}(A)}{6} \cdot \operatorname{Tr}^{3}
$$

$$
=U^{\prime}(A) W+U(A)
$$

der Quotient aus dem gesamten Ressourceneinsatz für RentSeeking und der politischen Rente als

$$
\lim _{n \rightarrow \infty} \frac{W}{\operatorname{Tr}}=1+\frac{U^{\prime \prime}(A)}{2 U^{\prime}(A)} \cdot \operatorname{Tr}+\frac{U^{\prime \prime \prime}(A)}{6 U^{\prime}(A)} \cdot \operatorname{Tr}^{2}
$$

bestimmt. Durch geeignete Erweiterung läßt sich diese Beziehung in Form von

$$
\lim _{n \rightarrow \infty} \frac{W}{\operatorname{Tr}}=1+\frac{A U^{\prime \prime}(A)}{2 U^{\prime}(A)} \cdot \frac{\operatorname{Tr}}{A}+\frac{U^{\prime \prime \prime}(A)}{6 U^{\prime}(A)} \cdot \operatorname{Tr}^{2}
$$

darstellen.

1) Zur Approximation von Funktionen mittels der Taylor'schenReihenentwicklung vgl. z.B. Henderson/Quandt (1983), S. $395 \mathrm{ff}$. 
Definiert man das von Arrow/Pratt (1964) entwickelte Maß der "relativen Risikoaversion" ${ }^{1}$ ) als

$$
r=-\frac{A U^{\prime \prime}(A)}{U^{\prime}(A)} \text {, }
$$

können die Konsequenzen unterschiedlicher Risikoeinstellungen der Konkurrenten im Wettbewerbsprozeß im Hinblick auf die Höhe des gesamten gesellschaftlichen Ressourceneinsatzes bestimmt werden. Danach ist Risikoneutralität durch einen Wert von $r$ gleich null gekennzeichnet, während bei Risikoaversion (Risikofreude) $r$ einen Wert von größer (kleiner) null annimmt. Aufgrund dieses Zusammenhanges kann (III.28) zu

$$
\lim _{n \rightarrow \infty} \frac{W}{T r}=1-r \cdot \frac{T r}{2 A}
$$

umgeformt werden. Aus dieser Beziehung ergibt sich die Schlußfolgerung, daß lediglich im Fall von risikoneutralem Verhalten das Ergebnis der "vollständigen Rentenabschöpfung" Gültigkeit behält. Bei risikoaversem Verhalten resultiert eine Verminderung des Quotienten von Ressourceneinsatz zu Monopolrente, während die entsprechenden Investitionen im Fall eines risikofreudigen Verhaltens den Gegenwartswert des Monopolprivilegs überschreiten ${ }^{2}$ ).

1) Vgl. Hey (1979), S. 48 bzw. Atkinson/Stiglitz (1980), S. 126. Eine ausführliche Diskussion dieser Maßzahl findet sich bei Pratt (1964), S. 122 ff. bzw. Arrow (1970), S. $90 \mathrm{ff}$. Zur Interpretation des Risikoaversionsmaßes muB die zugrundeliegende Nutzenfunktion streng monoton wachsen.

2) Vgl. Hillman/Katz (1984), S. 110. Unter Einbeziehung von Rent-Avoidance-Aktivitäten auf seiten der benachteiligten Individuen wird der soziale Ressourceneinsatz den Transferbetrag übersteigen. Vgl. Appelbaum/Katz (1986a), S. $175 \mathrm{ff}$. und Wenders (1987), S. $456 \mathrm{f}$. 


\section{Allokative Konsequenzen des Rent-Seeking}

Als wesentliche Konsequenz der Rent-Seeking-Argumentation ergibt sich, daß im Rahmen der Konkurrenz um staatlich ermöglichte Monopolrechte knappe Ressourcen "are engaged in competitive efforts that determine nothing than distributive results"1). Die für Umverteilungszwecke eingesetzten Ressourcen stellen eine volkswirtschaftliche Verschwendung dar, weil sie der alternativen Verwendung zur Steigerung der gesamtwirtschaftlichen Produktion entzogen werden.

Dieses zentrale Resultat der Rent-Seeking-Analyse veranschaulicht Tullock ${ }^{2}$ in einfacher Weise am Beispiel von Diebstahlsaktivitäten. Interpretiert man Diebstahl lediglich als distributives Nullsummenspiel, bei dem der Verlust eines Individuums genau dem Gewinn eines anderen Wirtschaftssubjektes entspricht, entsteht kein sozialer Verlust. Unter ökonomischen Gesichtspunkten handelt es sich um einen unkompensierten Pauschaltransfer. Werden allerdings die Ressourcen zur Durchführung und Abwehr dieser Form der Umverteilung berücksichtigt, läßt sich diese Interpretation nicht mehr aufrechterhalten. Mit der Ausdehnung staatlicher Aktivitäten werden zunehmend Anreize geschaffen, auf legalem Wege Distributionsvorteile zu erlangen. In Analogie zu den bekannten "HarbergerKosten" wurde für den durch Rent-Seeking hervorgerufenen Wohlfahrtsverlust die Bezeichnung "Tullock-Kosten" ${ }^{3}$ ) geprägt.

1) Buchanan (1980c), S. 359.

2) Vgl. Tullock (1967), S. 228 ff. aber auch Lee/McKenzie (1987), S. 48 .

3) Vgl. Rowley/Tollison (1986), S. 145 bzw. Wenders (1987), S. 456 . 
Zur Illustration ${ }^{1)}$ der Konsequenzen des Rent-Seeking wird in Abbildung 4 von einem fixen Ressourcenbestand einer ökonomie ausgegangen, der zur Produktion der Güter $x_{1}$ und $x_{2}$ verwendet wird. Die Produktionsmöglichkeitenkurve $x_{1}^{\circ} x_{2}^{\circ}$ stellt damit die maximal erreichbaren Outputkombinationen der Güter dar, wenn die verfügbaren Ressourcen ausschließlich zu Produktionszwecken in Form des Profit-Seeking eingesetzt werden. Die Möglichkeit des Rent-Seeking führt zum Ressourceneinsatz für Umverteilungsvorhaben (y).

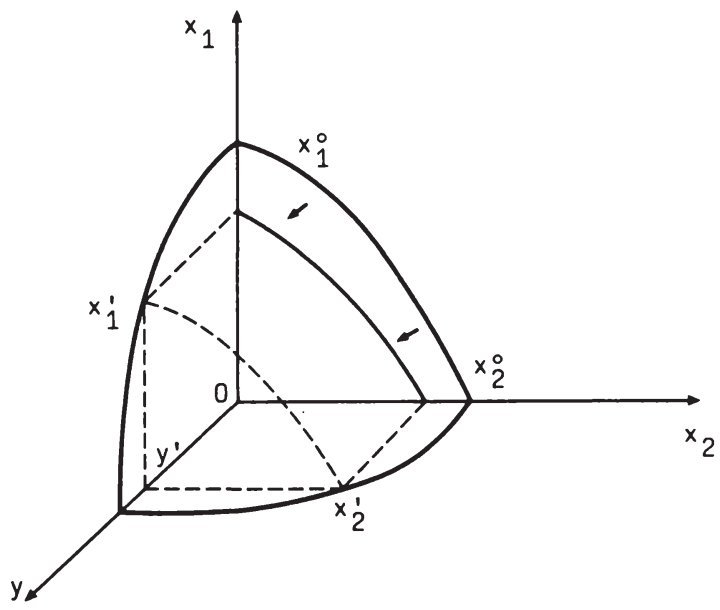

Abb. 4: Rent-Seeking und gesellschaftliche Produktionsmöglichkeiten

Mit dem Einsatz von $y^{\prime}$ Einheiten für Rent-Seeking ergibt sich der maximal mögliche Produktionsoutput durch die Produktionsmöglichkeitenkurve $x_{1}^{\prime} x_{2}^{\prime}$. Projiziert man diese Kurve zum Vergleich mit der Situation ohne Rent-Seeking-Aktivitäten in die

1) Vgl. in Verbindung mit der Analyse des Schmuggelns Sheikh (1974), S. 357 aber auch Brooks/Heijdra (1988), S. 30. 
Ebene der ursprünglichen Produktionsmöglichkeitenkurve, resultiert eine Verschiebung in Richtung zum Ursprung 0 . Die im Transferprozeß aufgewendeten Ressourcen "are purely wasteful from the standpoint of the society as a whole, they are spent not in increasing wealth, but in attempt to transfer or resist transfer of wealth"1). Auf der Grundlage dieser Erkenntnis muß eine vollständige Erfassung der mit staatlichen Aktivitäten verbundenen Wohlfahrtsverluste neben der resultierenden Mehrbelastung auch die volkswirtschaftlichen Verluste des RentSeeking einbeziehen ${ }^{2}$. Hinzu kommen weitere Effizienzverluste, wenn sich in Folge der erfolgreichen Realisierung von Monopolprivilegien eine Abweichung von der Minimalkostenkombination $\left(x\right.$-Ineffizienz) ergibt ${ }^{3}$.

Aufgrund der Ergebnisse des Grundmodells fand der Begriff RentSeeking Verwendung zur Charakterisierung eines Verhaltens "in institutional settings where individual efforts to maximize value generate social waste rather than social surplus"4). Je mehr Ressourcen in Transfervorhaben investiert werden, um so eher wandelt sich eine ökonomie zu einer Rent-SeekingSociety, einer Gesellschaft, die sich in zunehmendem Maße auf die Realisierung leistungsunabhängiger Einkommen - die sogenannten politischen Renten - verlegt ${ }^{5}$.

1) Tullock (1967), S. 228.

2) "A more general welfare analytics will include traditional deadweight costs, rent-seeking costs, and rent-protecting costs." Tollison (1987), S. 151.

3) Diese Erkenntnis kann als Erklärung für die zahlreichen Forderungen zur Aufrechterhaltung protektionistischer staatlicher Regelungen zur Unterstützung unrentabel gewordener Produktionsverfahren dienen. Der Aspekt der X-Ineffizienz wird im weiteren vernachlässigt. Vgl. dazu Crain/Zardkoohi $(1980)$, S. $784 \mathrm{f}$.

4) Buchanan (1980a), S. 4.

5) Vgl. Schmidtchen (1988), S. 15. 
Die Analyse der regulierenden Interventionen ${ }^{1)}$ des Staates läßt sich auch für die Beurteilung der Allokationswirkungen fiskalischer Institutionen nutzen ${ }^{2}$. Die grundlegende Argumentation soll am Beispiel der Einführung einer Lump-Sum-Stever auf eine reine Rente ${ }^{3)}$ dargestellt werden. Dabei wird von einem gegebenem Steueraufkommen ausgegangen, so daß durch die Steuerinstitution keine allokative Verzerrung zwischen privatem und öffentlichem Sektor erfolgt.

Die Neutralitätseigenschaft dieser Form der Besteverung beruht auf der Tatsache, daß ökonomische Entscheidungen nicht verzerrt werden und die für die Mehrbelastung verantwortlichen Substitutionseffekte entfallen. Aus der Rent-Seeking-Perspektive geht allerdings mit der Besteverung der Charakter einer reinen Rente verloren, da sich Opportunitätskosten in Höhe der Steuerbelastung ergeben. Die distributiven Konsequenzen der steverlichen Maßnahme induzieren politische Aktivitäten zur Vermeidung der damit verbundenen Nachteile (Rent-Avoidance) ${ }^{4}$ ). Daraus resultieren Allokationsverluste, die erhebliche Implikationen für die Ergebnisse der "Theorie der Optimalbesteverung" besitzen ${ }^{5}$ ).

1) Vgl. Demsetz (1984) bzw. Colander (1985), S. $199 \mathrm{ff}$.

2) Vgl. Lee (1985b), S. $731 \mathrm{ff}$. und Brooks/Heijdra (1987), S. $335 \mathrm{ff}$.

3) Beispielsweise wurde die Bodenrente als Bemessungsgrundlage für eine Alleinsteuer vorgeschlagen, weil das Angebot an Boden als Produktionsfaktor unelastisch reagiert und damit Mehrbelastungen vermieden werden. Vgl. Musgrave/Musgrave/ Kullmer (1985), S. 101.

4) "There is no such thing as a free tax." Lee/Tollison $(1985)$, S. 451.

5) Zum Konzept der "optimalen Besteuerung" vgl. Wiegard (1982), S. 155 ff. bzw. Mann (1987), S. 9 ff. 
Die Möglichkeit zu politischen Reaktionen von seiten der Belasteten bedeutet, daß die allokative Neutralität der Lump-SumSteuer nur aufrechterhalten werden kann, wenn neben einer vollständig unelastischen Angebotsfunktion der Bemessungsgrundlage ein politisch passives Verhalten der Steverzahler unterstellt wird.

Im realitätsnahen fall einer speziellen Verbrauchsteuer auf ein bestimmtes Gut werden bei normalen Nachfrage- und Angebotsbeziehungen sowohl Konsumenten als auch Produzenten von der Stever belastet. Unterstellt man, daß sich Produzenteninteressen leichter organisieren lassen als Konsumenteninteressen, werden die Produzenten ihren Rentenverlust durch politische Einflußnahme $z u$ vermeiden suchen ${ }^{1)}$. Der Allokationsverlust besteht aus der Mehrbelastung sowie dem Ressourceneinsatz für die Opposition. Während die Theorie der Optimalbesteuerung zur Minimierung der Mehrbelastung eine erhöhte Besteuerung von Gütern mit relativ unelastischer Nachfrage nahelegt $\left.{ }^{2}\right)$, ist unter Berücksichtigung des Rent-AvoidanceVerhaltens auch das umgekehrte Ergebnis denkbar. Sind politische Reaktionen bei einer Stever auf Güter mit unelastischer Nachfrage zu erwarten, kann sich eine erhöhte Besteuerung von Gütern mit elastischer Nachfrage als allokativ vorteilhaft erweisen, wenn hier keine politischen Aktivitäten hervorgerufen werden ${ }^{3)}$. Obwohl sich eine größere Mehrbelastung ergibt, ist der gesamte Allokationsverlust aufgrund der vermiedenen Kosten des politischen Widerstandes geringer.

1) Vgl. z.B. Downs (1968), S. 249 f.

2) Zur Inversen-Elastizitäten-Regel vgl. Baumol/Bradford $(1970)$, S. 272 .

3) Dieses Resultat hängt von den jeweiligen Angebots- und Nachfrageelastizitäten auf dem betrachteten Markt ab. Vgl. dazu Lee/Tollison (1988), S. $339 \mathrm{ff}$. 


\section{Interpretation und Kritik der Rent-Seeking-Konzeption}

Der im Grundmodell illustrierte Zusammenhang bildet den Ausgangspunkt der Kontroverse um die geeignete Charakterisierung des beschriebenen Sachverhaltes. Dabei wird von den Vertretern der sogenannten DUP-Theorie als Definition die Bezeichnung "directly unproductive profit-seeking (DUP) activities"1) vorgeschlagen. Gemäß dieser Begriffsbildung bedeuten solche Aktivitäten "ways of making a profit (i.e., income) by undertaking activities which are directly unproductive; that is, they yield pecuniary returns but do not produce goods that enter a utility function directly or indirectly via increased production or availability to the economy of goods that enter a utility function"2).

Da diese Aussage empirisch wahr oder falsch sein kann, stellt sie nur dann eine zulässige Definition dar, wenn die Analyse des Untersuchungsgegenstandes abgeschlossen ist. Wissenschaftliche Definitionen sollen der Erkenntnisgewinnung dienen, sie können selbst jedoch keine Erkenntnis beinhalten. Die wesentliche Funktion der Bildung von Definitionen besteht darin, begriffliche bzw. Semantische Unbestimmtheiten zu beseitigen und Bedeutungen festzulegen ${ }^{3}$ ).

1) Vgl. Bhagwati (1982a), S. 988 ff. bzw. Bhagwati/Srinivasan (1982), S. $33 \mathrm{ff}$. , Anam (1982), S. $15 \mathrm{ff}$. Oder Bhagwati (1983), S. $634 \mathrm{ff}$. und Bhagwati/Brecher/Srinivasan (1984), S. $17 \mathrm{ff}$. Zu einem Überblick über den DUP-Ansatz sei auf Bhagwati (1987), S. $845 \mathrm{ff}$. verwiesen. Vgl. auch die Diskussion zwischen Bhagwati (1980) bzw. (1982b) und Tullock (1981).

2) Bhagwati (1982a), S. 989.

3) Vgl. dazu Folkers (1987), S. $95 \mathrm{f}$. 
Die Integration der "Verschwendungshypothese" in die Definition des Untersuchungsgegenstandes stellt das Paradebeispiel für den häufig anzutreffenden Fehler dar, Definitionen und Ergebnisse wissenschaftlicher Analysen zu vermischen ${ }^{1}$. Mit dieser Definition wird Rent-Seeking a priori als Verschwendung charakterisiert, d.h. der Ressourceneinsatz ist damit per definitionem unproduktiv. Aufgrund dieser Argumentation ist der DUP-Ansatz unter methodischen Aspekten als verfehlt abzulehnen ${ }^{2)}$.

Ausgangspunkt der Rent-Seeking-Argumentation ist die Hypothese, "waste arises from the fact that resources which could be employed creating social value in some sector of the economy are used instead to procure wealth transfers" ${ }^{3)}$. Zur Untersuchung der allokativen Effekte des Akquisitionswettbewerbs erscheint daher die Beschränkung auf die Analyse des Marktes, in dem durch eine begünstigende Maßnahme des Staates Rentengewinne anfallen, nur begrenzt geeignet. Eine adäquate Interpretation der Rent-Seeking-Konzeption muß darüber hinaus die Effekte des Ressourceneinsatzes für Lobbying untersuchen.

1) Vgl. zu einem ähnlichen Beispiel die traditionelle "überwälzungshypothese" bei der Analyse von Verbrauchsteuern, die häufig bereits in der Definition des Begriffs "Verbrauchsteuer" auftaucht. Da die Steuerwirkungen den Gegenstand von Inzidenzanalysen bilden, kann die überwälzungshypothese grundsätzlich nicht Bestandteil einer Definition sein. Vgl. dazu Folkers (1987), S. $95 \mathrm{f}$.

2) Zur Diskussion der DUP-Konzeption sei auf North (1984), $\mathrm{S}$. $33 \mathrm{ff}$. und Rowley (1988a), S. $15 \mathrm{ff}$. verwiesen. Zur Kritik vgl. Brooks/Heijdra (1988), S. 28 ff. Allerdings muß die Rent-Seeking-Konzeption teilweise der gleichen Kritik unterzogen werden. So wird Rent-Seeking als "the activity of wasting resources in competing for artificially contrived transfers" definiert. Tollison (1982), S. 577. Vgl. auch Hartle (1983), S. 539 ff. und Pasour Jr. (1987), S. $123 \mathrm{ff}$.

3) Brooks/Heijdra (1988), S. 33. 
Zu diesem Zweck wird das Grundmodell der Rent-Seeking-Analyse erweitert ${ }^{1)}$. Um die interventionsbedingte Monopolrente im Markt des Gutes $x$ zu realisieren, muß ein Gut y erworben werden, das als Input für die Lobby-Aktivitäten der Interessenten beim Wettbewerb um das Monopolprivileg interpretiert werden kann. Die dadurch induzierten Effekte auf dem Markt für Gut $y$ werden in Abbildung 5 veranschaulicht ${ }^{2}$.

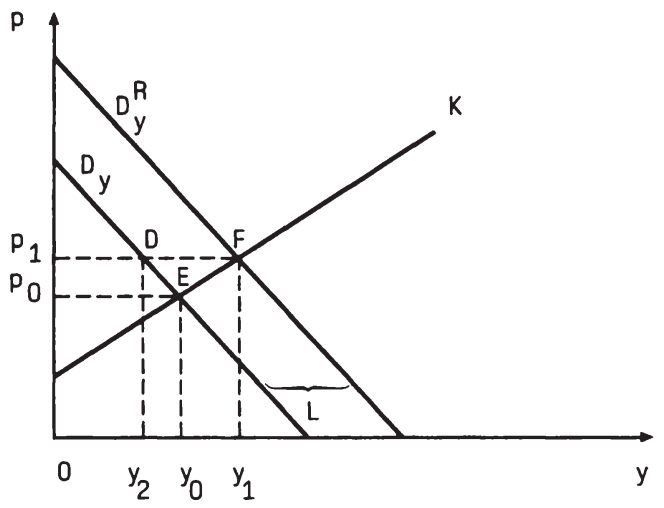

Abb. 5: Die Effekte des Ressourceneinsatzes für Lobbying

Aufgrund der Rent-Seeking-Aktivitäten zur Erlangung des Monopolprivilegs im Markt von $x$ (Abbildung 3 ) ergibt sich eine Nachfrageerhöhung auf dem Markt des Gutes y (Abbildung 5 ).

1) Vgl. Varian (1983) und Brooks/Heijdra (1988).

2) Diese Perspektive führt zu der Erkenntnis, daß "indviduals withdraw resources from some sector of the economy, and spend these resources on rent-seeking activities without at the same time expanding the output of the sector they wish to enter". Brooks/Heijdra (1988), S. 32. 
Dabei wird unterstellt, daß sich die Nachfragefunktion nach den notwendigen Mengeneinheiten von y für Lobby-Zwecke ( $L$ ) in der Form

( III.31)

$$
D_{y}^{R}= \begin{cases}L, & \text { wenn } \operatorname{Tr}>P_{y} L \\ 0, & \text { sonst }\end{cases}
$$

darstellen läßt ${ }^{1}$.

Die Berücksichtigung dieser Nachfrage für Lobby-Zwecke führt zur horizontalen Verschiebung der gewöhnlichen Nachfragefunktion $D_{y}$ um $L$, solange die Bedingung

$$
p^{\star} \leqq \frac{T r}{L}
$$

erfüllt ist. Mit $p^{*}$ wird der Preis für das Gut y bezeichnet, bei dem keine Nachfrage mehr nach y für Rent-Seeking-Zwecke entfaltet wird, da die Lobby-Ausgaben den Wert der durch die Realisierung der begünstigenden Regelung bei $x$ erzielbaren Renten $^{2)}$ übersteigen ${ }^{3)}$.

Als Konsequenz der Einbeziehung der für Lobbying erforderlichen Mengen des Gutes y ergeben sich entsprechende Preisund Mengenreaktionen auf diesem Markt. Mit der resultierenden Preiserhöhung auf $p_{1}$ ist eine Verminderung der für Konsumzwecke zur Verfügung stehenden Menge auf $y_{2}$ verbunden.

1) Vgl. Varian (1983), S. 26.

2) Der Rentengewinn im Markt von Gut $x$ wird in Abbildung 3 durch die Fläche $\mathrm{P}_{1} A B \mathrm{p}_{0}$ wiedergegeben.

3) Vgl. Varian (1983), S. 13. 
Eine wesentliche Erkenntnis des allgemeinen Gleichgewichtsmodells ist die Tatsache, daß sich mit dem Rent-Seeking-Verhalten ein Rentenzuwachs für die Produzenten dieses LobbyGutes ergibt, der sich in Gestalt von $P_{0} E F p_{1}$ darstellen läßt ${ }^{1}$. Gleichzeitig ist mit der Preiserhöhung ein Verlust an Konsumentenrente $\left(p_{1} D E p_{0}\right)$ verbunden, so daß ein Nettozuwachs in Höhe der Fläche DEF nachbleibt. Wenngleich die Fiktion eines LobbyGutes als Instrument zur Analyse von Rent-Seeking-Aktivitäten lediglich heuristischen Zwecken dient, ergibt sich die Möglichkeit einer Differenzierung der Ergebnisse des Grundmodells.

In dem Ausmaß, in dem durch Rent-Seeking eine Rentensteigerung bei den Anbietern des Gutes y hervorgerufen wird, kann der Ressourceneinsatz nicht als Verschwendung bezeichnet werden ${ }^{2}$ ). Die tatsächlichen Ausgaben für Lobbying werden von den Angebotsbedingungen auf dem Markt des Lobby-Gutes determiniert, während die maximale Zahlungsbereitschaft durch die Höhe der Verteilungsgewinne auf dem Markt von $x$ bestimmt wird. Aufgrund dieser Zusammenhänge stellt sich der gesamte Allokationsverlust als Summe der Mehrbelastung $A B C$ im regulierten Markt für das Gut $x$ (Abbildung 3 ) und der Fläche $y_{2} D E F y_{1}$ im Markt für das Lobby-Gut y (Abbildung 5) dar. Lediglich im Falle konstanter Grenzkosten des Angebotes des für Lobbying notwendigen Gutes y entspricht der soziale Verlust den Opportunitätskosten der Ausgaben für Rent-Seeking.

1) Zur ausführlichen Darstellung der Vorgehensweise zur Bestimmung der Wohlfahrtseffekte anhand veränderter Konsumentenbzw. Produzentenrenten vgl. Boadway/Wildasin (1984), S. $34 \mathrm{ff}$.

2) Die Verschwendungshypothese kann nur dann uneingeschränkt Gültigkeit beanspruchen, wenn der "expended effort has no value outside of obtaining the monopoly right". Higgins/ Tollison (1988), S. 149. 
Existieren auf dem Markt des Gutes y allokative Verzerrungen, können aufgrund der Erkenntnisse der Theorie des Second-Best ${ }^{1}$ ) entsprechende Rent-Seeking-Aktivitäten auf dem Markt von $x$ wohlfahrtssteigernd sein. Geht man allerdings davon aus, daß in aller Regel Informationsdefizite bezüglich der relevanten Parameter auf dem Markt von y vorliegen, sind Allokationsverbesserungen allenfalls zufällig und daher zu vernachlässigen ${ }^{2}$ ).

Darüber hinaus muß der Prozeß der Privilegvergabe bei der Analyse der allokativen Konsequenzen beachtet werden. "The degree to which government monopoly rents are dissipated depends on the type of government mechanism used to assign monopoly rights" ${ }^{3)}$. Es stellt sich die Frage, warum staatliche Instanzen (Politiker/Bürokraten) für die Gewährung begünstigender Monopolprivilegien zugunsten einzelner Wirtschaftssubjekte entscheiden sollten, wenn sie selbst keine Nutzensteigerungen $^{4}$ ) aus dem Rent-Seeking-Verhalten der Interessenten realisieren könnten. Werden die staatlichen Privilegien unter den Interessenten versteigert, sichert sich der Stat den vollen Wert der Monopolrenten ${ }^{5}$ ).

1) Vgl. Lipsey/Lancaster $(1956 / 57)$, S. $11 \mathrm{ff}$. bzw. im gegebenen Zusammenhang Buchanan (1980b), S. $183 \mathrm{ff}$. und Bhagwati $(1983)$, S. 636 .

2) Vgl. Brennan/McGuire (1975), S. $207 \mathrm{ff}$.

3) Higgins/Tollison (1988), S. 152 bzw. Congleton (1988), S. $181 \mathrm{ff}$.

4) In diesem Fall nimmt der Ressourceneinsatz für Rent-Seeking den Charakter einer Umverteilungsmaßnahme zugunsten der jeweiligen politischen Entscheidungsträger an.

5) Ebenso könnte die Distribution der handelbaren Rechte (z.B. bestimmter Lizenzen) nach dem Zufallsprinzip vorgenommen werden. 
Aufgrund der Transformation der Sonderrechte in marktich handelbare private Eigentumsrechte unterbleibt das bisher analysierte Rent-Seeking-Verhalten. Die Tatsache, daß die mit der Gewährung von Monopolprivilegien verbundenen Gewinne dem Staat zufallen, induziert Anreize zur Realisierung von Positionen im öffentlichen Sektor, die Zugang $z u$ diesen Renten eröffnen. "If the salaries and perquisites of government positions contain elements of economic rent"1) im Sinne einer höheren Entlohnung als in vergleichbaren Positionen des privaten Sektors, werden Individuen Ressourcen aufwenden, um sich als Politiker oder Staatsbedienstete diese "politischen Einkommen" 2 ) zu verschaffen.

Insbesondere ermöglicht die Existenz eines Monopolstaates in der Gestalt eines Leviathan Renteneinkommen in hohem AusmaB ${ }^{3}$ ). Mit dem Begriff des "Leviathan" wird ein "einnahmenverschlingender" Staat bezeichnet, der hinsichtlich der Vergabe von Privilegien eine Monopolstellung besitzt und daher die mit der Intervention verbundene Rente selbst abschöpfen kann ${ }^{4}$.

1) Buchanan (1980a), S. $13 \mathrm{f}$.

2) Zum Begriff des "politischen" Einkommens vgl. Buchanan $(1975)$, S. 157 .

3) Vgl. zum Monopolmodell des Staates insbesondere Brennan/ Buchanan (1980/1988). In gleicher Weise resultiert beispielsweise aus der staatlichen Besteuerungsgewalt ein gewisses Renteneinkommen, wenn die Steuereinnahmen nicht in vollem Umfang zur Bereitstellung der vom Steuerzahler gewünschten öffentlichen Güter verwendet werden müssen.

4) Vgl. Brennan/Buchanan (1980), S. 32. In diesem Kontext analysieren Ekelund Jr./Tollison (1981) bzw. (1984) den Aufstieg und Niedergang des Merkantilismus in England bzw. Frankreich. Vgl. ebenso Baysinger/Ekelund Jr./Tollison $(1980)$, S. $235 \mathrm{ff}$. bzw. Anderson/Tollison (1984), S. $187 \mathrm{ff}$. sowie Böbel (1988), S. $198 \mathrm{f}$. und Tullock $(1988 \mathrm{c})$, S. $409 \mathrm{ff}$. 
Der Wettbewerb um diese Positionen im öffentlichen Sektor ${ }^{1)}$ wird sich in erhöhten Ausbildungsinvestitionen der Interessenten niederschlagen. Da nur eine bestimmte Anzahl von Individuen diese begünstigenden Stellungen einnehmen kann, erfolgt eine unproduktive Bildung von Humankapital zu distributiven Zwecken. Aufgrund der dieser Arbeit zugrundeliegenden Definition des Untersuchungsgegenstandes soll diese Form des Wettbewerbs allerdings nicht als Rent-Seeking klassifiziert werden. Der Grund für diese Ausgrenzung liegt darin, daß keine Veränderung der Handlungsrechte anderer Wirtschaftssubjekte erfolgt ${ }^{2}$ ).

Wenn ein bestimmtes Auswahlverfahren die allgemeingültige Regel repräsentiert, stellt das individuelle Maximierungsverhalten unter dieser Regel keine Umverteilung von Rechten dar. Im Unterschied dazu beinhaltet jede auf politischem Wege induzierte Veränderung von Qualifikationserfordernissen für bestimmte Tätigkeiten Elemente des Rent-Seeking. Seiner Ausbildung entsprechend verfügt jedes Individuum über ein Fähigkeitspotential, aus dem es in Abhängigkeit von Art und Einsatz seines Humankapitals unterschiedliche Erträge erzielen kann. Die Rent-Seeking-Aktivitäten ergeben sich aus der Tatsache, daß mit Hilfe des Staates bestimmte Reglementierungen möglich sind, die zu einer Einschränkung der Nutzung von Bildungsinvestitionen führen und damit die individuellen Ertragsmöglichkeiten mindern. Beispielsweise werden steigende Zulassungsanforderungen für Ärzte teilweise zu Recht mit dem Schutz der Patienten vor unfähigen Ärzten gerechtfertigt.

1) Vgl. dazu Tullock (1980a), S. 16 ff., der die Konkurrenz um staatliche Positionen in China beschreibt.

2) Ricketts (1987), S. 462 bzw. auch Sisk (1985), S. 95 ff. sowie Dilorenzo (1988), S. 326 f. oder Buchanan (1983), S. $71 \mathrm{ff}$. 
Allerdings fällt auf, daß die Prüfungen lediglich neven Anwärtern abverlangt werden, obwohl sich mit dem gleichen Argument auch bereits praktizierende Ärzte regelmäßigen Prüfungen unterziehen müßten, um eine Betreuung nach den nevesten medizinischen Erkenntnissen zu gewährleisten ${ }^{1}$ ). Die Zielsetzung von Rent-Seeking-Vorhaben liegt hier in einer Entwertung des Humankapitals anderer Individuen durch gesetzliche Maßnahmen, da die politisch durchgesetzten Qualifikationserfordernisse die Funktion von Marktzutrittsbarrieren auf dem Arbeitsmarkt erfüllen, die den Zustrom von Konkurrenten verhindern und damit Renten sichern ${ }^{2)}$.

Im Zuge der Weiterentwicklung der Rent-Seeking-Hypothese wurden zahlreiche weitere Problembereiche ${ }^{3)}$ unter institutionellen Aspekten betrachtet. Erste empirische Untersuchungen basieren auf dem Modell des kompetitiven Rent-Seeking. Krueger ${ }^{4}$ ) beziffert den Anteil der Renten - und damit den Allokationsverlust - aus der Realisierung von Importlizenzen am Bruttosozialprodukt (GNP) von Indien im Jahre 1964 auf 7,3\% und von der Türkei im Jahre 1968 auf 15\%. Den Wohlfahrtsverlust beim Akquisitionswettbewerb um Monopolprivilegien schätzt Posner ${ }^{5}$ ) auf $3,4 \%$ des Bruttosozialproduktes der Vereinigten Staten.

1) Vgl. zu weiteren Beispielen 0lson (1982), S. 66.

2) Zur Problematik des Rent-Seeking im Arbeitsmarkt vgl. Meyer $(1987)$, S. 885 f.

3) Vgl.Z.B. Brennan/Tollison (1980), Laaser (1983), Baldwin (1984), Faminow/Benson (1984), Dnes (1985), Benson/Faminow (1988), Katz/Smith (1988), Grampp (1989) und Levy (1989).

4) Vgl. Krueger (1974) bzw. Mohammad/Whalley (1984), S. $387 \mathrm{ff}$.

5) Vgl. Posner (1975). Zu weiteren empirischen Ergebnissen in diesem Zusammenhang vgl. Cowling/Mueller (1978), S. $736 \mathrm{ff}$. bzw. Katz/Rosenberg (1989), S. 133 ff. oder Pittman (1988), S. $173 \mathrm{ff}$. 


\section{Zusammenfassung}

Ausgangspunkt der Rent-Seeking-Theorie ist die Hypothese, daß mit staatlichen Eingriffen in den Marktmechanismus bestimmte Distributionsvorteile zugunsten einzelner Wirtschaftssubjekte verbunden sind. Die durch staatliche Protektion erzielbaren Renten sind das Resultat statlicher Institutionen in Form von Marktzutrittsschranken, Kontingentierungen und zahlreicher weiterer Sonderregelungen, die Schutz vor unerwünschter Konkurrenz bzw. marktlichen Substitutionswirkungen bieten sollen. Die Zielsetzung der Rent-Seeking-Vorhaben liegt vorwiegend darin, durch vorteilhafte Ausnahmeregelungen von den allgemeingültigen wettbewerblichen Institutionen den Marktprozeß außer Kraft zu setzen und daverhafte Rentengewinne zu sichern.

Als zentrales Resultat der Analyse ist festzuhalten, daß die sozialen Kosten staatlicher Interventionen in den Marktmechanismus in traditioneller Sichtweise unterschätzt werden. Die im Distributionswettbewerb eingesetzten Ressourcen stellen eine volkswirtschaftliche Verschwendung dar, da sie der alternativen Verwendung zur Steigerung des gesamtwirtschaftlichen Output entzogen werden. Neben den mit allen nichtneutralen staatlichen Maßnahmen verbundenen Wohlfahrtsverlusten in Form der Mehrbelastung müssen zusätzlich die Allokationsverluste des Rent-Seeking einbezogen werden. Die herkömmliche Analyse beschränkt sich in erster Linie auf die Problematik der geeigneten Bemessung der Wohlfahrtsverluste beim Wettbewerb um einen in seiner Höhe exogen vorgegebenen Transferbetrag. Auf der Grundlage des Modells des kompetitiven Rent-Seeking wurden die Determinanten des individuellen und gesellschaftlichen Ressourceneinsatzes beim Akquisitionswettbewerb um staatlich gewährte "Monopolprivilegien" diskutiert. 
Der Umfang der investierten Ressourcen wird wesentlich von der Anzahl der Beteiligten am Distributionskonflikt und vom AusmaB des zur Disposition stehenden Transferbetrages bestimmt. Der Allokationsverlust entspricht lediglich dann der Höhe des Umverteilungsgewinns, wenn konstante Ertragszuwächse eines zunehmenden Ressourceneinsatzes in bezug auf die Steigerung der individuellen Erfolgswahrscheinlichkeit vorliegen und ein risikoneutrales Verhalten der Konkurrenten unterstellt wird. Die regulierungsinduzierten Renten werden unter diesen Bedingungen vollständig im Wege des kompetitiven Rent-Seeking-Prozesses aufgezehrt. In anderen Konstellationen kann der Wohlfahrtsverlust den Transferbetrag über-bzw. unterschreiten, während bei unvollständiger Information der Konkurrenten über die Höhe der Monopolrente eine Approximation der im Akquisitionswettbewerb verschwendeten Ressourcen nicht mehr möglich ist. Die Interpretation der Ergebnisse im Rahmen eines erweiterten Modells erlaubt eine differenzierte Beurteilung der RentSeeking-Konzeption und eine Abgrenzung vom DUP-Ansatz.

Ein Kritikpunkt an der bisherigen Analyse besteht allerdings darin, daß lediglich das kompetitive Verhalten von Individuen bei der Konkurrenz um einen exogen bestimmten Transferbetrag untersucht wird. Zur Analyse der Frage, welche Faktoren das Ausmaß des Verteilungsvorteils determinieren, müssen neben den Rent-Seeking-Aktivitäten der Begünstigten auch die Effekte der Abwehrmaßnahmen von seiten der benachteiligten Individuen berücksichtigt werden. Die Möglichkeit der Einflußnahme auf institutionelle Entscheidungen des Staates durch einzelne gesellschaftliche Gruppen kann einen Interessenwettbewerb im politischen Prozeß in Gang setzen. 


\section{ZUR BILDUNG UND FUNKTIONSWEISE VON INTERESSENGRUPPEN}

Die Einflußnahme auf staatlich determinierte Institutionen ist ein charakteristisches Merkmal der Aktivitäten von Interessengruppen ${ }^{1)}$. Die Zielsetzung der organisierten Interessenvertretung besteht darin, die gruppenspezifischen Interessen der Mitglieder zu artikulieren und im politischen Entscheidungsprozeß zur Geltung zu bringen. Zu diesem Zweck werden die Interessengruppen Ressourcen aufwenden, um durch die Ausübung politischer Einflußnahme ihre jeweiligen Distributionsvorhaben durchzusetzen. Da die Distributionsgewinne einer Gruppe in aller Regel zu Lasten anderer Gruppierungen gehen, wird Widerstand auf seiten der Verlierer induziert. Die Effektivität der Opposition ist allerdings davon abhängig, inwieweit es den Betroffenen gelingt, sich ihrerseits zu Interessengruppen zusammenzuschließen und ihre Gruppeninteressen zu artikulieren.

In diesem Kapitel wird die Frage der Bildung und Funktionsweise von Interessengruppen untersucht. Dabei werden Interessengruppen als Produzenten gruppenspezifischer Verteilungsvorteile interpretiert, die durch Produktion politischen Drucks eine Erhöhung ihres Einflusses im politischen Prozeß anstreben. Ob bestimmte Distributionsvorhaben durchgeführt werden können, hängt allerdings davon ab, inwieweit potentielle Gruppenmitglieder zur Aufbringung der erforderlichen Ressourcen beitragen. Neben den direkten Kosten der Einflußnahme im Informationsbereich und für Lobbying sind daher auf vorgelagerter Ebene die Kosten der Gruppenbildung von Bedeutung.

1) Zu einem Überblick über die Klassifikation, Aktivitäten und Funktionsweise von Interessengruppen sei auf die Beiträge von Werner (1965), Liefmann-Keil (1969), v. Beyme (1980) sowie den Sammelband von Blümle/Schwarz (1985) verwiesen. 


\section{Interessengruppen und fiskalische Gruppeninteressen}

Im Zentrum der individuellen Rent-Seeking-Aktivitäten bei fiskalischen Entscheidungen des Staates steht das Bemühen "to secure differentially favorable treatment, defined, not in terms of particular opportunities, but in terms of treatment by governmental fiscal process"1). Die Wirtschaftssubjekte werden Ressourcen in die Suche nach unzulänglichen Definitionen und Lücken in der unübersehbaren Fülle von Gesetzen und Vorschriften investieren, die Ansprüche auf einzelne Transferleistungen oder die Höhe der individuellen Steverzahlung bestimmen ${ }^{2}$ ).

Neben den Versuchen zur Erlangung von direkter und indirekter finanzieller Unterstützung durch den Staat werden auch Aktivitäten zur vorteilhaften Veränderung von Steuersätzen, Steuertarifen oder Bemessungsgrundlagen bzw. anderer fiskalischer Institutionen unternommen, um durch "begünstigende Korrekturen an historisch gegebenen Steuer/Ausgaben-Kombinationen"3) Verteilungsvorteile ) zu realisieren. Zur Durchsetzung ihrer fiskalischen Interessen werden Ressourcen zur Bildung bzw. Unterstützung von Interessengruppen aufgebracht, die durch politische Einflußnahmeaktivitäten die Gruppeninteressen gegenüber anderen Gruppen sowie den politischen Entscheidungsträgern vertreten sollen.

1) Buchanan (1980a), S. 14.

2) Auf dieser Ebene versuchen die Individuen "to become members of the groups favored by the tax-expenditure program". Tollison (1982), S. 579.

3) Folkers (1983a), S. 194.

4) Verteilungsvorteile ergeben sich "through the state budget in terms of expenditure increases and/or tax reductions" zugunsten bestimmter gesellschaftlicher Gruppen. Tollison (1982), S. 579 . 
In freiheitlich demokratischen Gesellschafts- und Wirtschaftssystemen ist die organisierte Interessenvertretung sowohl in normativer als auch in positiver Hinsicht zu begründen. Während sich die normative Rechtfertigung aus dem im Grundgesetz verankerten Recht der Meinungs- und Koalitionsfreiheit ergibt, resultiert die positive Begründung der Existenz von Interessengruppen aus ihrer Informationsfunktion im politischen Willensbildungsprozeß. Da alle am Entscheidungsprozeß beteiligten Akteure nur unzureichende Informationen besitzen, kommt den Interessengruppen zentrale Bedeutung bei der Beschaffung und Verarbeitung von entscheidungsrelevanten Informationen zu.

Interessengruppen vermitteln Informationen ${ }^{1)}$ sowohl an ihre Mitglieder bzw. Wähler als auch an Politiker und tragen daher zu einer Verminderung des Ressourcenaufwandes ${ }^{2}$ ) für Informationsgewinnung und Konsensfindung im politischen Prozeß bei. Allerdings ist $z u$ berücksichtigen, daß die verschiedenen Interessengruppen ${ }^{3}$ ) häufig divergierende bzw. widerstreitende Interessen vertreten. Interessengegensätze bei ein und derselben staatlichen Entscheidung sind das charakteristische Merkmal von Distributionskonflikten ${ }^{4}$ ).

1) Beispielsweise besitzen Parteien und Regierung häufig keine Kenntnis der Wählerpräferenzen bzw. haben nur geringe Informationen über die Konsequenzen beabsichtigter bzw. bereits verabschiedeter staatlicher Maßnahmen. In gleicher Weise sind die Wähler nur unzureichend über die sie betreffenden Effekte informiert, die mit bestimmten politischen Entscheidungen verbunden sind. Vgl. Bernholz (1969), S. 282 f. bzw. (1974), S. 45 ff.

2) Vgl. Streit (1986b), S. 199 bzw. (1988a), S. 47.

3) Zur Pointierung der Zielrichtung der Vorhaben dieser Gruppen wird auch die Bezeichnung "Verteilungskoalitionen" verwendet. Vgl. Olson (1982), S. 44.

4) Vgl. Pethig (1986), S. 145. 


\section{Interessengruppen als Produzenten von Verteilungsvorteilen}

Die Einflußnahme auf politische Entscheidungen unterliegt dem gleichen ökonomischen Kalkül wie jede Investitionsentscheidung. Individuen bzw. ihre Vertreter werden die erwarteten Distributionsgewinne den Einflußnahmekosten gegenüberstellen und abwägen, inwieweit ein weiterer Ressourceneinsatz zur Steigerung des politischen Einflusses noch zu Verteilungsgewinnen führt. Zur Illustration der Entscheidungssituation wird von der Vorstellung identischer Gruppenmitglieder ausgegangen, so daß die Analyse auf das Maximierungsverhalten eines repräsentativen Mitglieds der betrachteten Gruppe beschränkt werden kann. Im folgenden wird zunächst die gruppeneffiziente ${ }^{1)}$ Produktion von politischem Einfluß ermittelt. Anschließend werden die Determinanten der Entscheidung für eine bestimmte Produktionstechnik untersucht.

\subsection{Bestimmung des gruppeneffizienten Produktionsniveaus von politischem EinfluB}

Das aktive Bemühen um partikulare Distributionsvorteile kommt in der Produktion von politischem Einfluß zum Ausdruck. "The production of influence... involves attempts by agents to increase their real incomes by altering relevant decisions made in the public sphere"2). Aus der Sicht der jeweiligen Gruppen ist diejenige Produktion politischen Einflusses effizient, die den Verteilungsgewinn für die Mitglieder maximiert.
1) Vgl. Pethig (1986), S. 146
2) Bartlett (1973), S. 144. 
Der politische Einfluß einer Interessengruppe wird durch

(IV.1) $\quad I=I(p)$

wiedergegeben, wobei $p$ die Menge des produzierten politischen Drucks bezeichnet ${ }^{1)}$ und

(IV.2) $\quad \frac{d I}{d p}>0 \quad$ bzw. $\quad \frac{d^{2} I}{d p^{2}}<0$

gilt.

Die Produktionsfunktion des politischen Drucks zur Steigerung des Einflusses ergibt sich als

(IV.3) $\quad p=p(R)$

und wird durch die für politische Aktivitäten zur Verfügung stehende Ressourcenmenge R bestimmt. Gemäß

(IV.4) $\frac{d p}{d R}>0 \quad b z w . \quad \frac{d^{2} p}{d R^{2}}<0$

werden abnehmende Ertragszuwächse des Ressourceneinsatzes bei der Produktion politischen Drucks unterstellt. Die Kosten der politischen Einflußnahme bestehen aus dem Ressourceneinsatz für die Bildung und Aufrechterhaltung von Interessengruppen und den Kosten für Lobbying und Beeinflussungen im Informationsbereich.

1) Zum Konzept polıtischer Einflußnahmefunktionen sei auf Bartlett (1973), S. $144 \mathrm{ff}$. bzw. Becker (1983), S. $375 \mathrm{ff}$. bzw. Balisacan/Roumasset (1987), S. 234 verwiesen. 
Diese politischen Einflußnahmekosten werden durch die Funktion (IV.5) $\quad C=C(R)$

wiedergegeben ${ }^{1}$ ), wobei

(IV.6) $\frac{d C}{d R}>0 \quad b z w . \quad \frac{d^{2} C}{d R^{2}}>0$

gilt. Dieser Kostenverlauf weist darauf hin, daß es zunehmend aufwendiger wird, weitere politische Renten durchzusetzen ${ }^{2}$.

Die Zielfunktion der Interessengruppe läßt sich in Form von (IV.7)

$$
G=I[p(R)]-C(R) \rightarrow \max !
$$

darstellen, wobei $G$ den Gewinn aus der politischen Einflußnahme bezeichnet. Aus dieser Beziehung wird die notwendige Bedingung als

$$
\frac{d G}{d R}=\frac{d I}{d p} \cdot \frac{d p}{d R}-\frac{d C}{d R}=0
$$

ermittelt. Der optimale Ressourceneinsatz ergibt sich, wenn die Grenzerträge politischer Einflußnahme den Grenzkosten entsprechen. Dieser Zusammenhang wird in Abbildung 6 dargestellt.

1) Zum Instrumentarium politischer Einflußnahme sei auf Bernholz (1969), S. 283 f., Bartlett (1973), S. 132 ff. bzw. Breton (1974), S. 74 ff. oder Bernholz/Breyer (1984), S. 349 ff. verwiesen.

2) Vgl. auch Bartlett (1973), S. 146 und Frey (1985), S. 20. 


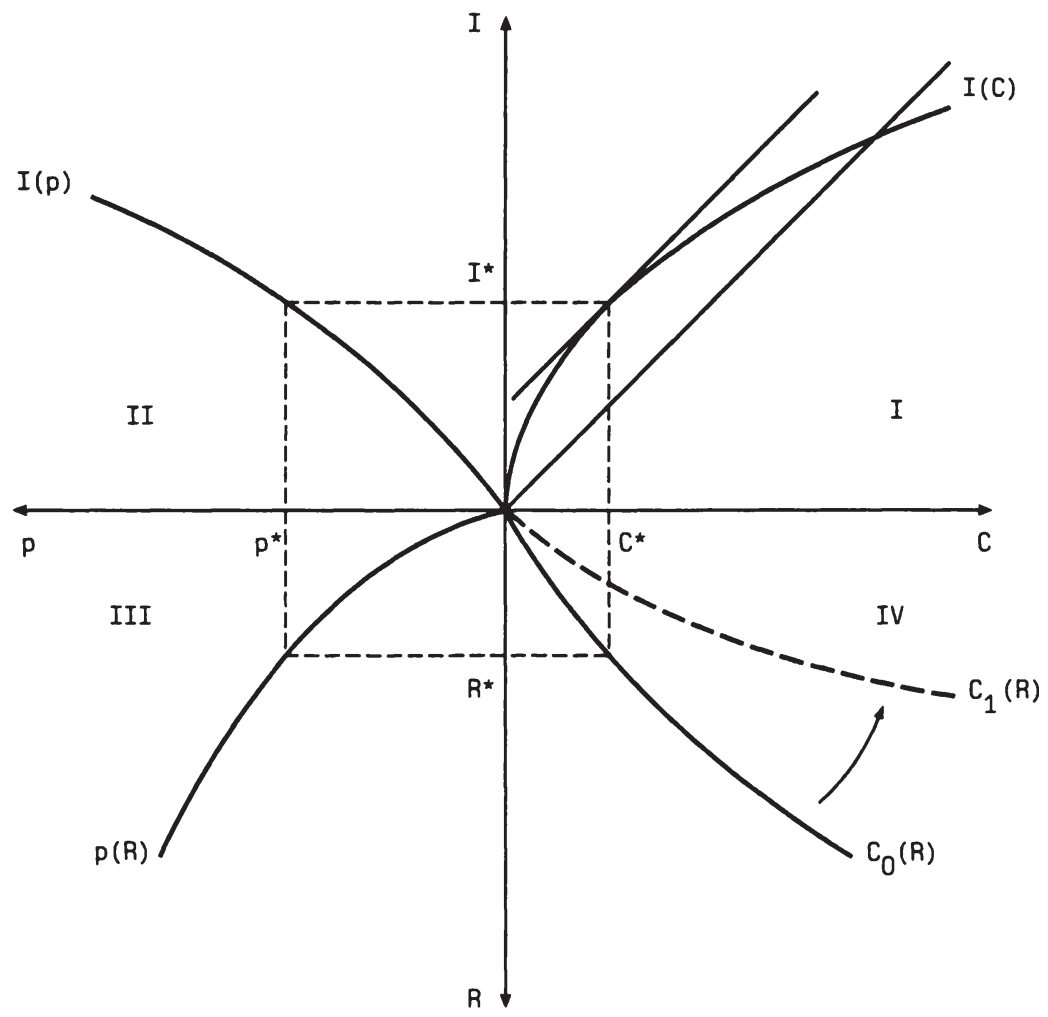

Abb. 6: Die Kosten und Nutzen politischer Einflußnahme

In Quadrant IV ${ }^{1)}$ sind die Einflußnahmekosten in Abhängigkeit des Rent-Seeking-Ressourceneinsatzes abgetragen. Die Produktionsfunktion politischen Drucks wird in Quadrant III dargestellt, während die dadurch induzierte Einflußnahme in

1) Vgl. dazu Wellisz/Findlay (1984), S. 143 bzw. McCormik/ Tollison (1981), S. 37. Ähnliche Darstellungen finden sich bei Bartlett (1973), S. 146 und Frey (1985), S. 20. 
Quadrant II durch Transformation in Quadrant I als Funktion der Rent-Seeking-Kosten abgebildet werden kann. Der optimale Ressourceneinsatz ergibt sich gemäß (IV.7) bei $R^{\star}$.

Eine entscheidende Determinante des politischen Gewichts einer Gruppe ist die Fähigkeit, sich zu organisieren und die für den politischen Verteilungskampf notwendigen Ressourcen aufzubringen. Sind die Interessen nur schwer organisierbar, kann sich eine Konstellation ergeben, in der sich die Entfaltung politischer Einflußnahme nicht lohnt. Wenn sich aufgrund hoher Organisationskosten die Kostenfunktion $C_{0}(R)$ ceteris paribus verschiebt (vgl. $C_{1}(R)$ in Quadrant IV von Abbildung 6), liegt die Einflußnahmefunktion $I(C)$ im gesamten Bereich unterhalb der Winkelhalbierenden ${ }^{1)}$. Daher werden keine Ressourcen für politische Aktivitäten aufgewendet. Verfügt die Gruppe dagegen über eine gute Organisation, d.h. sind die Anfangskosten der Organisation der Interessen bereits erbracht, wird eine politische Einflußnahme lohnend. Diese Überlegung stellt eine Begründung für die Tatsache dar, daß bereits existierende Interessengruppen ihre Anliegen im politischen Bereich erfolgreicher verfolgen, während neu zu bildende Interessengruppen ihre Vorhaben nur schwerer durchsetzen können ${ }^{2)}$. Als Konsequenz dieser Überlegungen ist festzuhalten, daß die Ausübung politischer Einflußnahme - und damit die Durchführung von Distributionsvorhaben - entscheidend von den Kosten der Gruppenbildung ${ }^{3)}$ determiniert werden.

1) Vgl. Bartlett (1973), S. 147

2) So kann beispielsweise die Interessengruppe aus anderen Gründen bereits bestehen, so daß im konkreten Fall die Organisationskosten nicht ins Gewicht fallen.

3) Diese Kosten bestehen neben den reinen Organisationskosten aus den Kosten der Vermeidung des "Trittbrettfahrer"-Verhaltens, die im wesentlichen von der Gruppengröße abhängen. Vgl. Folkers (1983a), S. $196 \mathrm{ff}$. 


\subsection{Bestimmung der gruppeneffizienten Produktionstechnik}

Aus der unterschiedlichen Ausstattung einzelner Interessengruppen hinsichtlich der Art der zur Verfügung stehenden Ressourcen lassen sich Aussagen über die Einflußnahmetechniken ableiten. Prinzipiell kann zwischen geld- und zeitintensiven Formen der Beeinflussung unterschieden werden ${ }^{1}$ ). Bezeichnet man die im Produktionsprozeß eingesetzten Zeitressourcen bzw. monetären Ressourcen mit $R_{T} b z w$. $R_{M}$, kann die Entscheidung für eine bestimmte Einflußnahmetechnik anhand von Abbildung 7 illustriert werden. Auf der Ordinate ist die monetäre Ressourcenmenge abgetragen, während auf der Abszisse die vorhandene Menge an Zeitressourcen abgelesen werden $\mathrm{kann}^{2}$ ). Die Steigung der Isokostengerade wird durch das Verhältnis der relativen Preise der Ressourcen bestimmt, wobei die Opportunitätskosten der Zeitressourcen durch den Lohnsatz bzw. der monetären Ressourcen durch den Kapitalertrag in alternativer Verwendung wiedergegeben werden ${ }^{3}$ ). Betrachtet werden zwei Gruppen s und $t$, die über eine unterschiedliche Ausstattung mit beiden Ressourcenarten verfügen. Während der Gruppe s in größerem Ausmaß Zeitressourcen zur Verfügung stehen, besitzt die Gruppe t mehr monetäre Mittel.

1) In analoger Weise wird im Rahmen der Produktionstheorie zwischen arbeits - und kapitalintensiver Produktionstechnik unterschieden.

2) Vgl. Breton (1974), S. 102 bzw. Becker (1965), S. 493 ff.

3) "A rational agent will make his choice on the basis of his relative endowments of the two inputs into the production process, and upon the opportunity costs he faces with regard to other uses of these inputs." Bartlett (1973), S. 147 . 


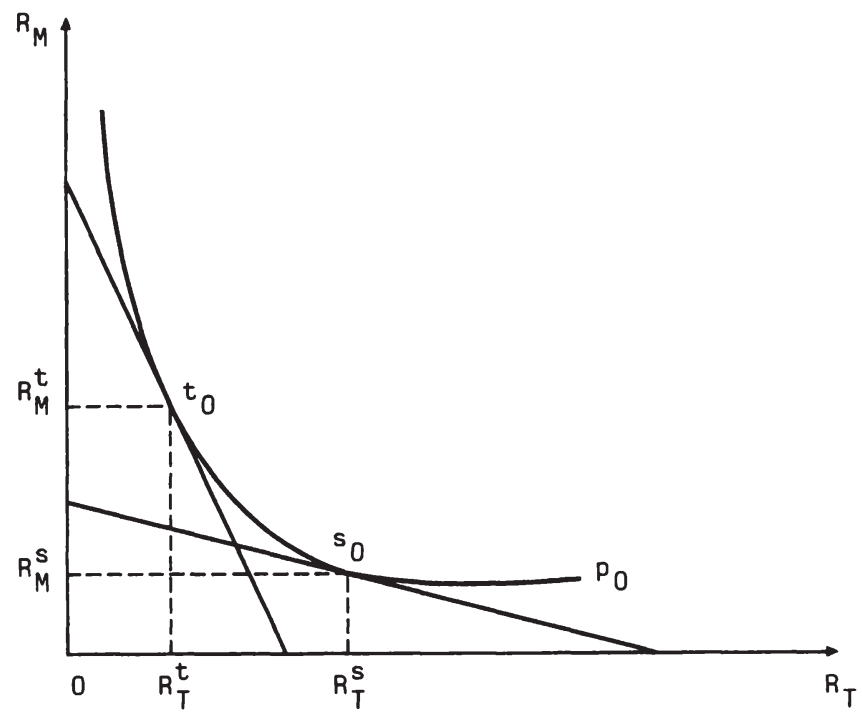

Abb. 7: Die Entscheidung zwischen alternativen Einflußnahmetechniken

Die Isoquante $p_{0}$ spiegelt eine mit verschiedenen Kombinationen monetärer und zeitlicher Ressourcen produzierbare Menge an politischem Druck wider. Aufgrund der divergierenden 0pportunitätskosten der jeweiligen Ressourcen bei beiden Gruppen resultiert eine zeitintensive Einflußnahmetechnik auf seiten von Gruppe s, während sich Gruppe $t$ für die geldintensivere Produktionstechnik entscheiden wird ${ }^{1}$. Damit ergeben sich die gruppenspezifischen Optimalpunkte $s_{0} b z w . t_{0}$ als Tangentialpunkte der gruppenbezogenen Isokostengerade mit der maximal erreichbaren Isoquante der Produktion politischen Drucks.

1) So ist beispielsweise die Teilnahme an einer Demonstration eine sehr zeitintensive Form der Beeinflussung, während die Einflußnahme in Form von Wahlspenden eher eine geldintensive Produktionsmethode darstellt. 


\section{Die Konsequenzen der gruppeninternen Interessenkon- stellation}

In der ökonomischen Analyse organisierter Interessenvertretung war lange Zeit das pluralistische Paradigmal) vorherrschend. Dabei wird die Gesellschaft als ein Netzwerk von Gruppen gesehen, deren Mitglieder sich freiwillig auf der Grundlage gemeinsamer Interessen zusammenschließen. Insbesondere wird davon ausgegangen, daß die gemeinsame Zielsetzung ausreicht, um die Gruppenbildung zu ermöglichen. Nach der Analyse von $01 \operatorname{son}^{2}$ ) ist allerdings zu konstatieren, daß der Zusammenschluß von Wirtschaftssubjekten mit gleichen Interessen nur unter spezifischen Voraussetzungen möglich ist. Die Grundproblematik der Organisation der Gruppeninteressen resultiert aus dem Umstand, daß der Gegenstand des gemeinsamen Interesses - sei es die Erhaltung, Abschaffung oder Veränderung einer bestimmten staatlichen Institution - Eigenschaften eines Kollektivgutes besitzt.

Das zentrale Problem der gruppeninternen Interessenkonstellation liegt in der Überwindung des "Prisoner's-Dilemma" ${ }^{3)}$, das sich als Konsequenz der Unteilbarkeit des Gutes "Politischer Einfluß" ergibt. Dabei handelt es sich um ein gruppenspezifisches Kollektivgut ${ }^{4}$, das im Hinblick auf eine spezielle Gruppe definiert ist, die sich durch ein gemeinsames Interesse an seiner Bereitstellung konstituiert. Allerdings haben zwangsläufig alle potentiellen Mitglieder der Gruppe die Möglichkeit, das Gut bzw. die daraus resultierenden Vorteile zu nutzen.

1) Vgl. dazu Bentley (1908) bzw. Truman (1971).

2) Vgl. Olson (1985), S. $14 \mathrm{ff}$. bzw. Olson (1987), S. $474 \mathrm{f}$.

3) Vgl. insbesondere Hardin (1982), S. $16 \mathrm{ff}$.

4) Vgl. Pethig (1986), S. $145 \mathrm{ff}$. 
Eine auf politischem Wege erzwungene Veränderung des institutionellen Umfelds zugunsten einer Interessengruppe kommt daher neben den Mitgliedern der Gruppe auch Nichtmitgliedern zugute. Da kein Individuum vom Ergebnis der Rent-Seeking-Aktivitäten ausgeschlossen werden kann, besteht wenig Anreiz, einer entsprechenden Gruppierung beizutreten und Ressourcen zur Unterstützung und Förderung der gemeinsamen Ziele aufzuwenden.

Angesichts dieses Zusammenhanges resultiert die Schlußfolgerung, daß sich große Gruppen mit gleicher Interessenlage nur dann organisieren lassen, wenn die Mitglieder neben den unteilbaren Kollektivgütern zusätzlich private Güter erhalten oder die Mitgliedschaft erzwungen werden kann. Mit der Gewährung von Zusatzleistungen ( $z$. B. in Form von Rechtsberatung, Gruppenversicherungen etc.) wird versucht, die Beitrittswilligkeit zu erhöhen, da diese Produkte nur Mitgliedern zugute kommen. Wenngleich die Schaffung "selektiver Anreize"1) ein gruppenorientiertes Handeln und damit die Bildung von Interessengruppen fördert, ist gleichwohl davon auszugehen, daß allgemeine Interessen latenter Gruppen nur schwer organisierbar sind. Im Fall kleiner Gruppen besteht hingegen eine merkliche Interdependenz der individuellen Verhaltensweisen. Solange das einzelne Individuum durch seinen Ressourceneinsatz erheblich zur Erlangung des kollektiven Gutes beiträgt, liegen günstige Voraussetzungen für eine Organisation der Gruppeninteressen vor. Damit besitzen relativ kleine Gruppen mit homogenen Interessen ${ }^{2}$ ) Vorteile beim "kollusiven" ${ }^{3)}$ Rent-Seeking.

1) Vgl. Olson (1985), S. $49 \mathrm{ff}$.

2) Vgl. auch Frey (1971), S. 101 ff. bzw. Russel (1972), $\mathrm{S} .113 \mathrm{ff}$. und Frey (1972), S. $119 \mathrm{f}$.

3) Der Begriff des kollusiven Rent-Seeking bezeichnet den Sachverhalt, daß "a group of agents colludes in order to obtain taxes, subsidies, tariffs and the like which will benefit all members of the group". Varian (1983), S. 20. 
Als Konsequenz des individuell rationalen "Trittbrettfahrer"Verhaltens ergeben sich insbesondere Auswirkungen auf die Ausgestaltung spezieller Rent-Seeking-Vorhaben ${ }^{1)}$. So ist $z u$ vermuten, daß in erster Linie spezifische Privilegien zugunsten begrenzter und gut organisierter Gruppen angestrebt werden, während politische Vorhaben mit generellen Distributionsvorteilen für alle Individuen unterbleiben ${ }^{2)}$. Beispielsweise werden einzelne Interessengruppen keine politischen Aktivitäten entfalten, um eine Minderung der nominellen Progression des Einkommenstevertarifs oder die Absenkung des Mehrwertsteuersatzes durchzusetzen ${ }^{3}$ ). Die Begründung liegt in der Tatsache, daß hier hohe Kosten der Organisation und der Überwindung des "Trittbrettfahrer"-Verhaltens anfallen.

Darüber hinaus würden den Rent-Seeking-Kosten der aktiven Gruppen im Fall der Realisierung allgemeiner Verteilungsvorteile nur geringe potentielle gruppenspezifische Erträge gegenüberstehen. Aufgrund dieser überlegungen ist davon auszugehen, daß die Vertreter der einzelnen Gruppen nur solche Sonderregelungen im politischen Prozeß anstreben, die speziell ihrer Klientel zugute kommen, während Vorhaben zur Realisierung allgemeiner Verteilungsvorteile kaum organisiert werden ${ }^{4}$.

1) Vgl. Folkers (1985), S. $277 \mathrm{f}$.

2) Diese Überlegung erklärt auch die Bemühungen der Gewerkschaften um institutionelle Entscheidungen (z.B. im Arbeitsrecht), die ausschließlich ihren Mitgliedern zugute kommen. Vgl. auch Watrin (1973), S. $85 \mathrm{f}$.

3) Die Dispersion der Vorteile wird sich jeweils nur "auf relativ kleine Gruppen beziehen, da die Organisationskosten und damit die Vermeidungskosten des free-rider-Verhaltens hier gering sind". Folkers (1983a), S. 196.

4) Vgl. dazu insbesondere die Diskussion zwischen Tullock (1971c), S. $629 \mathrm{ff}$. und Browning (1974), S. 375 sowie Tullock (1974a), S. $378 \mathrm{ff}$. 


\section{Die Bedeutung der Gruppenfunktionäre}

Um die Vorteile zu sichern, die eine Interessengruppe verspricht, müssen die Mitglieder Rechte bzw. Ressourcen an die Gruppenfunktionäre abtreten ${ }^{1)}$. Die wesentliche Bedeutung des Funktionärs einer Interessengruppe besteht darin, für potentielle Mitglieder Anreize zu schaffen, um diese zur Einbringung von Ressourcen für die Förderung der gemeinsamen Interessen zu veranlassen. Unter diesem Aspekt kann ein Gruppenfunktionär als "institutioneller Unternehmer"2) interpretiert werden, der ebenfalls ein Interesse an der Produktion des gruppenspezifischen Gutes "Einfluß" besitzt, weil er entweder aus dem Kollektivgut selbst oder aus dem Produktionsprozeß Nutzen (Macht, Prestige, finanzielle Vorteile etc.) zieht. Allerdings ist kaum anzunehmen, daß die Vertreter der Gruppen lediglich ausführende Organe der Gruppenwünsche darstellen. Wenngleich sie durch die Notwendigkeit der Bestätigung im Amt einer gewissen Kontrolle durch die Basis unterliegen, werden sie sich mit der Ausweitung ihrer Budgets diskretionäre Handlungsspielräume zur Erfüllung ihrer Eigeninteressen zu schaffen suchen. Um ihre Einflußnahmemöglichkeiten zu steigern, sind die Funktionäre an der Verleihung des Status der "Zwangsmitgliedschaft" 3 ) für die Gruppenangehörigen interessiert.

1) Zum Modell der Ressourcenzusammenlegung vgl. Coleman (1972) bzw. Vanberg (1982), S. $10 \mathrm{ff}$.

2) Der Begriff des "institutionellen Unternehmers" wird in Analogie zum Konzept des "politischen Unternehmers" gewählt, das vor allem von Wagner (1966), S. $161 \mathrm{ff}$. und Moe (1980) entwickelt wurde. Vgl. auch Salisbury (1969), S. $1 \mathrm{ff}$., Eschenburg (1975), S. $257 \mathrm{ff}$. und Frohlich/ Oppenheimer/Young (1971).

3) Dabei können staatliche Instanzen die Verleihung eines solchen Status den Gruppenfunktionären als Gegenleistung für ein bestimmtes politisch gewünschtes Verhalten der Gruppe anbieten. Auf diese Weise wird den Funktionären gleichzeitig die Überwindung der gruppeninternen Interessenkonstellation erleichtert. Vgl. Gäfgen (1987), S. 5 f. 
Durch Zwangsmitgliedschaft wird die "Exit-Steuerung"1) über den Mitgliedermarkt außer Kraft gesetzt. Darunter versteht man ein funktionierendes Wettbewerbssystem des Aus-bzw. Beitritts von Individuen, das ein Verhalten der Gruppenfunktionäre entsprechend den Wünschen der "Basis" fördert und damit eine Anpassung der Aktivitäten der Interessengruppen an die Mitgliederpräferenzen erzwingt ${ }^{2}$ ).

Die institutionellen Bedingungen der Organisation von Gruppeninteressen bestimmen damit im Zusammenwirken mit den Restriktionen der Gruppenfunktionäre das Ausmaß der Produktion von politischem Einfluß. Tendenziell lassen sich die Gruppenziele bei Zwangsmitgliedschaft besser verfolgen als bei freiwilliger Mitgliedschaft, da ein höherer Ressourcenbetrag für Einflußnahmeaktionen zur Verfügung steht ${ }^{3}$ ). Die Interessenvertretung bei der Organisation auf freiwilliger Basis impliziert aufgrund der gruppeninternen Interessenlage im Vergleich zur Konstellation bei Pflichtmitgliedschaft eine zu geringe Produktion politischen Drucks, die mit steigender Gruppengröße weiter abnimmt. Allerdings kann dieser komparative Nachteil durch Schaffung selektiver Anreize ${ }^{4}$ ) vermindert werden.

1) Vgl. dazu grundlegend "Abwanderung und Widerspruch" von Hirschmann (1974).

2) Vgl. Groser $(1980)$, S. $115 \mathrm{f}$.

3) Vgl. Pethig (1986), S. 150 f., der das Lobbying einer Interessengruppe um einen monetären Transferbetrag untersucht. Dabei wird die Produktion des gruppeneffizienten Umfangs politischer Einflußnahme als profitable Aktivität eines Gruppenfunktionärs erklärt, die von den institutionellen Bedingungen der Organisation der Gruppeninteressen (Pflichtmitgliedschaft bzw. freiwillige Mitgliedschaft) bestimmt wird. Vgl. ebenso Pethig (1987), S. $153 \mathrm{ff}$. bzw. (1988), S. $270 \mathrm{ff}$.

4) Vgl. 0lson (1985), S. 49 bzw. Pethig (1985), S. 69. 
Darüber hinaus ist die Wirksamkeit der Kontrolle eines abweichenden Funktionärsverhaltens von Bedeutung. Beispielsweise kann eine im Hinblick auf die Festlegung der Höhe der Beitragssätze unbeschränkte Verbandsführung bei Zwangsmitgliedschaft in monopolistischer Weise die Gruppe ausbeuten, deren "Interessen" sie vertritt. Wenngleich unter diesen Bedingungen die gruppeneffiziente Einflußnahmemenge produziert wird, ist das Einkommen eines repräsentativen Gruppenmitgliedes "nach dem Lobbying genauso groß wie ohne"1). D.h. durch die interessenorientierte Ausgestaltung der Beiträge wird der gesamte gruppenspezifische Distributionsvorteil zugunsten der Funktionäre abgeschöpft.

Ebenso ist aufgrund mangelnder Kontrollmöglichkeiten der Gruppenfunktionäre durch die Basis mit einer Abweichung von der Minimalkostenkombination bei der Produktion politischen Drucks zu rechnen. Diese X-Ineffizienz äußert sich beispielsweise in Gestalt kostspieliger Büroausstattungen, zusätzlicher Reiseaktivitäten und zahlreicher "unnötiger" Maßnahmen im Repräsentationsbereich, die nicht-monetäre Vorteile für die Funktionäre darstellen. Diese überlegungen zeigen, daß sich alternative Bedingungen der Organisation von Gruppeninteressen auf die Höhe der Verteilungsvorteile im Wettbewerb der Interessengruppen auswirken werden.

1) Pethig (1986), S. 147 . 


\section{Zusammenfassung}

Die Zielsetzung der organisierten Interessenvertretung liegt in der Realisierung staatlicher Maßnahmen, die im Interesse ihrer Mitglieder liegen und politische Renteneinkommen ermöglichen. Interessengruppen können als Produzenten von gruppenspezifischen Verteilungsvorteilen interpretiert werden, die mit der Produktion politischen Drucks eine Steigerung ihres Einflusses im staatlichen Entscheidungsprozeß anstreben.

Die gruppeninterne Interessenkonstellation resultiert aus der Problematik der Produktion des gruppenspezifischen Kollektivgutes "Einfluß". Kommt eine auf politischem Wege durchgesetzte Begünstigung neben den Mitgliedern auch Nichtmitgliedern zugute, wird die Bildung von Interessengruppen erschwert. Die Ursache liegt in dem rationalen "Trittbrettfahrer"-Verhalten der Individuen, die aufgrund der Kollektivguteigenschaft der Interessengruppenaktivitäten keinen Anreiz haben, Ressourcen zur Förderung der gemeinsamen Interessen aufzuwenden. Aus diesem Grund besitzen relativ kleine, nach spezifischen Merkmalen abgegrenzte Gruppen komparative Vorteile bei der Organisation der Gruppeninteressen. Als Konsequenz dieser Überlegungen ergibt sich eine Verminderung der Rent-Seeking-Vorhaben mit allgemeinen Wirkungen, allerdings keine generelle Reduktion der politischen Einflußnahmeproduktion von seiten der Interessengruppen.

In diesem Zusammenhang sind auch die Zielsetzungen und Restriktionen der Gruppenfunktionäre zu berücksichtigen, die sowohl das Ausmaß der Produktion von politischem Druck als auch die Wahl des Instrumentariums politischer Partizipation beeinflussen. Gelingt einer Gruppe eine schlagkräftige Organisation ihrer Interessen und sind die Kosten der Gruppenbildung gering, werden Distributionsvorhaben in zunehmendem Maße gruppenspezifisch ertragreich. 


\section{DER WETTBEWERB VON INTERESSENGRUPPEN UM POLITISCHEN EINFLUSS}

Im Rahmen dieses Kapitels wird das Zustandekommen fiskalischer Regelungen aus dem Wettbewerb von Interessengruppen um politischen Einfluß erklärt. Die Analyse der widerstreitenden Verteilungsinteressen erfolgt auf der Grundlage eines Gleichgewichtsmodells, wobei die konkurrierenden Bemühungen einzelner Interessengruppen zur Gewinnung von politischem Einfluß Art und Umfang der staatlichen Maßnahmen bestimmen ${ }^{1)}$. Dabei werden insbesondere die Effekte von interessenbedingten politischen Aktivitäten zur Änderung fiskalischer Regelungen im Zusammenwirken mit den marktlichen Anpassungsreaktionen der Gruppenmitglieder an gegebene Steuer-bzw. Ausgabeinstitutionen untersucht.

Ausgehend von der gruppenspezifischen Interessenantinomie bei Verteilungskonflikten wird zuerst auf die Determinanten des politischen Gleichgewichts eingegangen. Auf dieser Grundlage werden die Effekte einer Veränderung wesentlicher Bestimmungsfaktoren für das Gleichgewicht der Gruppeninteressen dargestellt. Zur Illustration der Konsequenzen des reinen Verteilungskampfes zwischen den Interessengruppen wird zunächst die Ebene der politischen Interessen vernachlässigt. D.h. die staatlichen Entscheidungsträger werden als "Transfermakler"2) interpretiert, die lediglich die aus dem Wettbewerb der Gruppen um politischen Einfluß resultierenden Maßnahmen ausführen. Den Abschluß bildet eine Zusammenfassung der wesentlichen Resultate.

1) Es wird von der Vorstellung ausgegangen, daß staatlich determinierte Institutionen "are used to raise the welfare of more influential pressure groups". Becker (1983), S. $373 \mathrm{f}$.

2) McCormik/Tollison (1981), S. 15. 


\section{Die Interessenantinomie beim Distributionskonflikt}

Interessengegensätze bei einer staatlichen Entscheidung sind das charakteristische Merkmal von Distributionskonflikten. Wenngleich auch staatliche Ausgabenprogramme unter distributiven Aspekten angestrebt werden, bildet insbesondere der Bereich staatlicher Steuerpolitik den Gegenstand widerstreitender Interessen, da die "Verteilungsregeln für fiskalische Allokationsentscheidungen nicht festgelegt sind, sondern in gewissen Grenzen zur Disposition stehen, d.h. daß zugleich allokative und distributive Ziele mit demselben Instrument verfolgt werden"1). Rent-Seeking-Aktivitäten richten sich auf die Realisierung von Steuerprivilegien ${ }^{2}$ ) durch eine vorteilhafte Ausgestaltung der Steuerstruktur, welche ihrerseits "the structure of opportunities within the economy and, thus, society" ${ }^{3)}$ bestimmt. Interessengruppen werden Bemühungen zur Änderung der Steuergesetze unternehmen und "Ressourcen aus produktiven Verwendungen in die Durchsetzung begünstigender Steverreformen umlenken"4).

1) Folkers (1983a), S. 194 .

2) Eine weitere Möglichkeit zur Realisierung von Steuervorteilen besteht in der Flucht in die "Schattenwirtschaft". Steigende Steuersätze induzieren Anreize, sich umfassender $z u$ informieren und höhere Risiken einzugehen, da der Ertrag in Form vermiedener Steuerzahlungen steigt. Obwohl diese Form der individuellen Steverminderung in den letzten Jahren an Bedeutung gewonnen hat, wird sie aufgrund der Eingrenzung des Rent-Seeking-Konzeptes auf die legale Form der Realisierung von Verteilungsvorteilen durch die Ausübung politischer Einflußnahme nicht weiter betrachtet. Zu Fragen der Abgrenzung und Messung der Schattenwirtschaft vgl. Pommerehne/Frey (1982), S. 157 f. bzw. Weck (1983).

3) Alt (1983), S. 184 .

4) Folkers (1985), S. 275 . 
Die Intensität der politischen Einflußnahmeaktivitäten wird durch die individuellen Gewinnmöglichkeiten bei einer Änderung der Steuerstruktur bestimmt. Aufgrund der Tatsache, daß steuerliche Maßnahmen gleichzeitig ${ }^{1)}$ die ökonomische Position mehrerer Individuen tangieren, werden die Verteilungsgewinne für bestimmte Individuen mit Belastungen anderer Wirtschaftssubjekte einhergehen ${ }^{2}$ ). Um die gegensätzlichen Interessenlagen beim Distributionskonflikt zu verdeutlichen, wird davon ausgegangen, daß die Steuervorteile in vollem Umfang durch eine entsprechende Besteverung der im Verteilungskampf unterlegenen Gruppe finanziert werden. Damit bleiben im gegebenen Zusammenhang mögliche Verminderungen des staatlichen Leistungsniveaus unberücksichtigt, d.h. es wird eine unveränderte Nutzenstiftung aus staatlichen Leistungen unterstellt ${ }^{3}$.

Als Maßstab zur Bestimmung der nutzenmäßigen Position eines repräsentativen Mitglieds einer solchen Gruppe dient sein sogenanntes "Full Income"4). Mit diesem Begriff wird das Einkommen bezeichnet, das ein Individuum realisieren könnte, wenn es die gesamten zur Verfügung stehenden Ressourcen zur Erzielung von Einkommen einsetzen würde.

1) Vgl. Tullock (1971a), S. 913.

2) Beispielsweise müssen einzelne Gruppen einen erhöhten Beitrag zur Aufrechterhaltung eines bestimmten Umfangs staatlicher Leistungen erbringen, wenn andere Gruppen sich im Wege des Rent-Seeking Stevervorteile sichern.

3) Eine geänderte Nutzenstiftung aus staatlichen Leistungen resultiert dann, wenn die Stevervorteile neben der Erhöhung der Stevern für andere Gruppen eine zumindest teilweise Reduktion des staatlichen Leistungsniveaus implizieren.

4) Eine ausführliche Diskussion dieses Einkommenskonzeptes findet sich bei Becker (1981), Kap. 1 und 4. 
Im Unterschied zur Verwendung des reinen marktlichen Einkommens erlaubt dieses umfassendere Einkommenskonzept eine einheitliche Behandlung aller individuellen Substitutionen zwischen nichtmonetären und monetären Einkommensbestandteilen sowie die Einbeziehung des monetären Äquivalents der Freizeit und anderer nichtmarktlicher Aktivitäten. Jede Veränderung der so spezifizierten Einkommensposition eines Gruppenmitglieds durch eine staatliche Maßnahme wird als politisch erzwungener Transfer ( $T r$ ) bezeichnet. Die Einkommensänderungen werden durch die staatliche Maßnahme und die dadurch induzierte Änderung des marktlichen Einkommens infolge von privaten Anpassungsreaktionen der Gruppenmitglieder bestimmt.

Zur Analyse der Problematik widerstreitender Distributionsinteressen wird von zwei homogenen gesellschaftlichen Gruppen ausgegangen, deren Mitglieder von der staatlichen Aktivität in gleicher Weise tangiert werden ${ }^{1)}$. Alle Transfers, die eine Minderung des individuellen Einkommens darstellen, werden als Stever (Distributionsverlust) bezeichnet, während in analoger Weise Einkommenserhöhungen als eine Steververgünstigung bzw. Subvention (Distributionsgewinn) interpretiert werden. Zur Vereinfachung der Darstellung der gruppenspezifischen Interessenkonstellation wird angenommen, daß die Wirtschaftssubjekte eindeutig der besteverten bzw. begünstigten Gruppe zugeordnet werden können ${ }^{2}$ ).

1) Damit wird von der Vorstellung identischer Gruppenmitglieder ausgegangen.

2) Obwohl Individuen in der Regel sowohl der Besteuerung unterliegen als auch Vergünstigungen erhalten, erscheint diese Annahme aus Gründen der deutlicheren Abgrenzung der jeweiligen Gruppeninteressen gerechtfertigt. 
Der effektive Umverteilungsvorteil für ein repräsentatives Mitglied der begünstigten Gruppe s läßt sich somit als Differenz

(V.1) $\quad \operatorname{Tr}^{s}=Y_{1}^{S}-Y_{0}^{s}$ mit $\operatorname{Tr}^{s}>0$

darstellen, wobei $Y_{1}^{S}\left(Y_{0}^{S}\right)$ die Einkommensposition nach (vor) der staatlichen Maßnahme symbolisiert.

In gleicher Weise wird der tatsächliche Distributionsnachteil für ein Mitglied der belasteten Gruppe $t$ als

(V.2) $\quad T^{t}=Y_{0}^{t}-Y_{1}^{t}$ mit $\operatorname{Tr}^{t}>0$

ermittelt. Auf dieser Grundlage lassen sich die gruppenspezifischen Zielsetzungen im Distributionskonflikt ableiten. Die Steuerzahler werden versuchen, ihre Gesamtsteuerlast zu mindern, während die Gruppe der Begünstigten eine Erhöhung ihres effektiven Verteilungsvorteils anstrebt.

\section{Die Handlungsmöglichkeiten der Gruppenmitglieder}

In traditioneller Sicht werden meist lediglich die marktlichen Anpassungsreaktionen an Veränderungen fiskalischer Institutionen untersucht. Unter Rent-Seeking-Aspekten müssen zusätzlich die politischen Einflußnahmeaktivitäten zur interessenbezogenen Ausgestaltung statlicher Regelungen einbezogen werden, um aus der Analyse des Zusammenwirkens beider Handlungsmöglichkeiten zu einer positiven Erklärung des individuellen bzw. des gruppenspezifischen Verhaltens bei Distributionskonflikten zu gelangen ${ }^{1)}$.

1) Vgl. Forte/Peacock (1981), S. $3 \mathrm{f}$. 


\subsection{Die Effekte marktlicher Anpassungsreaktionen}

Ausgangspunkt der Analyse der marktmäßigen Anpassungsreaktionen der von einer Stever in gleicher Weise betroffenen Individuen ist die Hypothese, daß diese Wirtschaftssubjekte in erster Linie eine Minderung ihrer steverlichen Gesamtbelastung anstreben ${ }^{1)}$. Der gesamte mit der steverlichen Maßnahme verbundene Nutzenverlust ( $\mathrm{Tr}^{\mathrm{t}}$ ) setzt sich aus der monetären Steuerzahlung und der Mehrbelastung zusammen, die durch die individuellen marktlichen Anpassungsreaktionen an die jeweilige steverliche Institution hervorgerufen wird ${ }^{2}$.

Diese Zusatzlast beruht auf den dabei induzierten Substitutionseffekten, die zur Verzerrung bisher effizienter privater Entscheidungen führen. Das Ausmaß der Mehrbelastung wird durch die Stärke der Verhaltensanpassung bestimmt und nimmt mit steigendem Marginalsteversatz $z^{3}{ }^{3}$. Allerdings sind die Individuen durchaus bereit, eine Erhöhung der Zusatzlast zu tolerieren, wenn auf diese Weise eine Verminderung der Gesamtbelastung möglich ist ${ }^{4}$ ). Unter Berücksichtigung dieser überlegungen ist mit einer individuellen Nutzeneinbuße in Höhe von $\operatorname{Tr}^{t}$ eine im Vergleich dazu geringere monetäre Steuerzahlung $F$ des belasteten Wirtschaftssubjektes verbunden.

1) Diese Hypothese des individuellen Verhaltens scheint realistischer als das aus normativer Sicht postulierte Ziel einer Minimierung der Mehrbelastung oder der Verwirklichung übergeordneter Gerechtigkeitsvorstellungen. $\mathrm{Vgl}$. Folkers (1983a), S. 191.

2) Darunter fallen alle "deadweight costs that result from the distorting effects on hours worked, investments, and other taxpayer choices". Becker (1983), S. 374.

3) Vgl. Harberger (1971), S. 785 ff.

4) Vgl. Folkers (1983a), S. 191. 
Damit ergibt sich

(V.3) $\quad F\left(T^{t}\right) \leqq T r^{t}$,

wobei

(V.4) $\quad F^{\prime}=\frac{d F\left(T^{t}\right)}{d T^{t}} \leq 1 \quad$ bzw. $\quad F^{\prime \prime}=\frac{d^{2} F\left(T^{t}\right)}{d T r^{t^{2}}} \leq 0$

gilt. Lediglich im Fall einer Pauschalsteuer werden die für die Mehrbelastung verantwortlichen Substitutionseffekte vermieden, so daß keine Allokationsverluste auftreten. Eine allokationsneutrale Form der Besteverung wird daher durch (v.5) $\quad F\left(T^{t}\right)=\operatorname{Tr}^{t}, \quad F^{\prime}=1$ und $\quad F^{\prime \prime}=0$ wiedergegeben ${ }^{1)}$. Da Individuen ihre Steuerzahlung in aller Regel durch marktmäßige Anpassungsreaktionen beeinflussen werden ${ }^{2}$, ergibt sich das Gesamtsteueraufkommen $T$ in Form von

$$
T=n^{t} F\left(T r^{t}\right)
$$

wobei $n^{t}$ die Anzahl der Mitglieder der Steverzahlergruppe symbolisiert.

1) Vgl. Becker (1983), S. 374 .

2) "The individual, therefore, may gladly tolerate some degree of excess burden if he can thus secure the adoption of a tax system which reduces his individual share of the cost of collective goods." Goetz (1964), S. 35. Vgl. auch Goetz (1978), S. 799. 
In gleicher Weise werden auf seiten der Begünstigten Anpassungsreaktionen an die jeweilige Regelung der Transfergewährung erfolgen, so daß ebenfalls Nutzenverluste in Form einer Mehrbelastung induziert ${ }^{1}$ ) werden. Müssen die Individuen bisher effiziente marktliche Entscheidungen zur Realisierung von Verteilungsvorteilen ändern, fallen die effektiven Nutzenzuwächse geringer aus als die entsprechenden Transferausgaben des Staates.

Aufgrund dieses Zusammenhangs läßt sich das zur Erzeugung eines individuellen Distributionsgewinns in Höhe von $\mathrm{Tr}^{\mathrm{s}}$ erforderliche Subventionsvolumen $G$ in Form von

(V.7) $\quad G\left(\operatorname{Tr}^{s}\right) \geq \operatorname{Tr}^{s}$

darstellen, wobei

$$
G^{\prime}=\frac{d G\left(T r^{s}\right)}{d T^{s}} \geqq 1
$$$$
\text { bzw. } \quad G^{\prime \prime}=\frac{d^{2} G\left(T r^{s}\right)}{d T r^{2}} \geq 0
$$

unterstellt wird. Nur im Fall von Pauschalsummentransfers entsprechen die staatlichen Transferausgaben in voller Höhe dem Distributionsvorteil des Begünstigten, so daß

$$
G\left(\operatorname{Tr}^{s}\right)=\operatorname{Tr}^{s}
$$$$
G^{\prime}=1
$$

und

$$
G^{\prime \prime}=0
$$

gilt ${ }^{2}$.

1) "A subsidy may also create efficiency losses, with the result being that the monetary amount of the subsidy overstates the net gain to the subsidized group." Coe/Wilber (1985), S. 41 bzw. auch Folkers (1985), S. 281.

2) Vgl. Becker (1983), S. 375 . 
Wenngleich die Gewährung von Vergünstigungen unter speziellen Bedingungen bestehende Ineffizienzen kompensieren kann, werden derartige Effekte im Zusammenhang mit Distributionszielsetzungen vernachlässigt. Dies erscheint realistisch, da bei Verteilungskonflikten die Allokationswirkungen in der politischen Auseinandersetzung überhaupt nicht diskutiert werden und Allokationsverbesserungen folglich nur durch Zufall auftreten können. Bei Unsicherheit über die Konsequenzen von Vergünstigungen sollte aufgrund unzureichender Informationen bezüglich der relevanten Parameter auf ihren Einsatz zur Realisierung von Allokationszielen verzichtet werden.

Die Begründung dieser Argumentation resultiert aus der Erkenntnis, daß der Erwartungswert der Allokationsverluste aus einer fehlgerichteten Abweichung vom Optimum bei Bedingungen vollkommener Konkurrenz den Erwartungswert der Allokationsgewinne übersteigt, wenn man gleiche Wahrscheinlichkeiten für das Eintreten beider Konstellationen annimmt ${ }^{1)}$. Da eine ausreichende Kenntnis der Wirkungszusammenhänge ${ }^{2}$ in aller Regel nicht unterstellt werden kann, ist von Nutzenverlusten auf seiten der Begünstigten auszugehen.

1) Vgl. Brennan/McGuire (1975), S. 207 bzw. Folkers (1985), S. 281 sowie Mann (1987), S. $170 \mathrm{f}$.

2) Da die Gewinnung der erforderlichen Informationen durch die Existenz von externen Effekten, Monopolen etc. erschwert wird, sind Richtung und Ausmaß der staatlichen Maßnahmen zur Korrektur unerwünschter Abweichungen vom Konkurrenzoptimum kaum zu bestimmen. Vgl. Folkers (1987), S. $170 \mathrm{ff}$. 
Zur Verdeutlichung des Distributionskonfliktes wird eine statliche Budgetrestriktion in Form von

$$
n^{t} F\left(T r^{t}\right)=T=s=n^{s} G\left(T r^{s}\right)
$$

eingeführt, wobei $n^{s}$ die Anzahl der Mitglieder der begünstigten Interessengruppe bezeichnet. Da der Budgetrestriktion eine zentrale Bedeutung beim politischen Wettbewerb um Distributionsvorteile zukommt, wird in diesem Zusammenhang auch der Begriff der "politischen Budgetgleichung"1) verwendet. Daraus folgt, daß die Höhe der Vergünstigungen $S$ durch das zur Verfügung stehende Steveraufkommen $T$ begrenzt wird. Darüber hinaus wird die grundlegende Interessenantinomie bei staatlichen Umverteilungsmaßnahmen zum Ausdruck gebracht, da jede Begünstigung der einen Gruppe vollständig zu Lasten der anderen Gruppe geht ${ }^{2}$ ).

Um die Beziehungen zwischen den effektiven Verteilungsgewinnen bzw. effektiven Verteilungsvorteilen und dem monetären Steveraufkommen bzw. Subventionsvolumen zu verdeutlichen, soll die Konstellation

$$
n^{t} T^{t}=n^{s} \operatorname{Tr}
$$

betrachtet werden. In diesem - allerdings unrealistischen Fall einer allokationsneutralen Besteverung bzw. Subventionsgewährung stimmen die monetären Zahlungsströme mit den tatsächlichen Nutzenverlusten bzw. -gewinnen beider Gruppen überein.

1) Diese Beziehung symbolisiert "the budget equation between the amount paid in taxes and the amount received as subsidies, a relation that has a major effect on the competition for political influence". Becker (1983), S. $375 \mathrm{f}$.

2) Vgl. Pethig (1986), S. 145 . 
Werden zusätzlich die im Verteilungskampf induzierten Mehrbelastungseffekte berücksichtigt, ergeben sich materielle Unterschiede $z$ wischen dem tatsächlichen Verteilungsnachteil (Verteilungsvorteil) und dem monetären Steueraufkommen (Subventionsvolumen). "Thus, the total cost of the tax will exceed the total gain from the subsidy as a result of efficiency losses from the transfer"1). Selbst bei effizienter Besteuerung und formalem Budgetausgleich werden die effektiven Verluste der belasteten Individuen die tatsächlichen Vorteile übersteigen, wenn auf seiten der Vergünstigungsempfänger Mehrbelastungseffekte auftreten.

Zur Illustration der Konsequenzen von marktlichen Anpassungsreaktionen der Steuerzahler bzw. der Vergünstigungsempfänger dient Abbildung 8 , in der die Beziehungen zwischen dem tatsächlichen Nutzenverlust bzw. Nutzenzuwachs und den Mehrbelastungseffekten für ein repräsentatives Mitglied der jeweiligen Gruppe veranschaulicht werden.
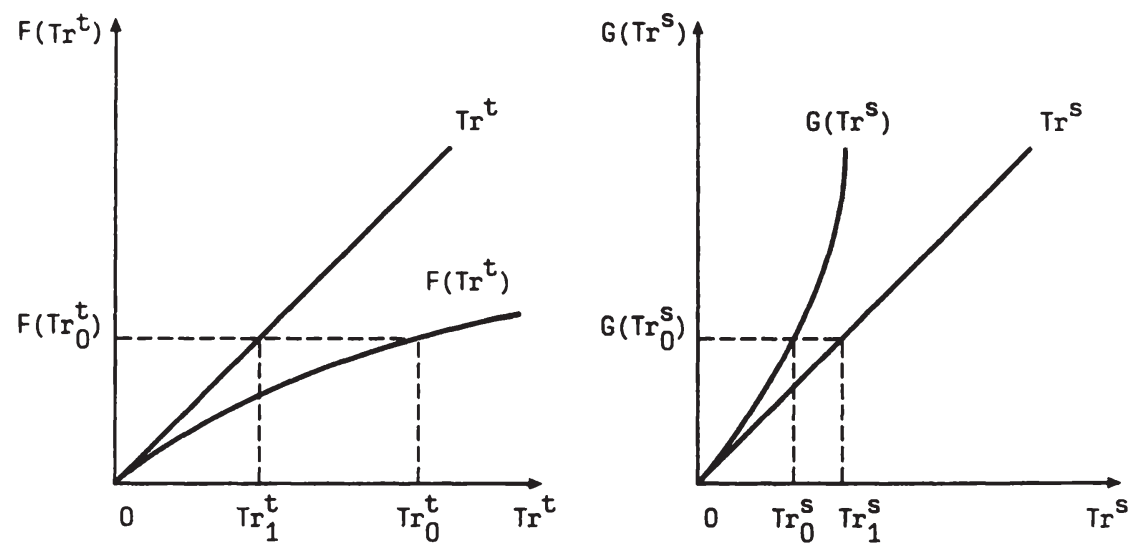

Abb. 8: Die Mehrbelastungseffekte von Steuern und Vergünstigungen

1) Coe/Wilber (1985), S. 41. 
Auf der Abszisse wird die Höhe der effektiven Belastung $\left(\operatorname{Tr}^{t}\right.$ ) bzw. Begünstigung ( $\operatorname{Tr}^{\mathrm{s}}$ ) abgetragen, während auf der 0rdinate die entsprechende monetäre Steverzahlung bzw. Vergünstigung abgelesen werden kann. Mit einer individuellen Nutzeneinbuße in Form von $\operatorname{Tr}_{0}^{t}$ ist ein Steueraufkommen $F\left(T_{0}^{t}\right)$ verbunden, das für Distributionszwecke zur Verfügung steht. Bei allokationsneutraler Besteuerung könnte das gleiche Aufkommen mit einer individuellen Belastung in Höhe von $\operatorname{Tr}_{1}^{t}$ realisiert werden. Aus diesem Grund kann das Ausmaß der Mehrbelastung als Abweichung von der neutralen Besteverung bei unverzerrten Allokationsentscheidungen durch die Differenz zwischen $\operatorname{Tr}_{0}^{t}$ und $\operatorname{Tr}_{1}^{t}$ bestimmt werden ${ }^{1 \text { ). }}$

In analoger Weise resultiert bei Gewährung einer Vergünstigung in Höhe von $G\left(\operatorname{Tr}_{0}^{S}\right)$ lediglich ein effektiver Verteilungsgewinn von $\operatorname{Tr}_{0}^{s}$, wenn sich das Individuum zur Erlangung des Vorteils mit seinen marktlichen Entscheidungen anpassen muß. Verglichen mit einer nicht verzerrenden Transferzahlung von gleicher Höhe, ergibt sich eine Nutzenkürzung gegenüber $\operatorname{Tr}_{1}^{s}$, d.h. der effektive Verteilungsvorteil vermindert sich für den Begünstigten um die Mehrbelastung. Der Anteil der Mehrbelastung am gesamten Distributionsvorteil ist davon abhängig, in welchem Ausmaß Verhaltensänderungen für die Realisierung des Verteilungsgewinns erforderlich sind ${ }^{2}$. Wird nur eine spezielle Interessengruppen unter spezifischen Bedingungen begünstigt, ergeben sich hohe Mehrbelastungskosten für die Begünstigungsempfänger. Aufgrund dieser "market effects" ${ }^{3)}$ sind die effektiven Verteilungsgewinne der Begünstigten geringer als die

1) Vgl. Folkers (1985), S. $282 \mathrm{f}$.

2) Zur Bemessung der Allokationsverluste vgl. Harberger (1971), S. 788 bzw. Buchanan/Brennan (1980), S. $41 \mathrm{ff}$.

3) Dieser Begriff wird von Bartlett (1973), S. 88 für die Wirkungen der privaten Anpassungsreaktionen an staatich determinierte Institutionen gewählt. 
staatlichen Transferausgaben, während der Nutzenverlust auf seiten der belasteten Wirtschaftssubjekte das zur Finanzierung der Vorteile notwendige Steueraufkommen übersteigt.

Somit ist festzuhalten, daß alle nichtneutralen staatlichen Aktivitäten, die aus der Verfolgung distributiver Partikularinteressen resultieren, zu Nutzenverlusten bei den am Distributionskonflikt beteiligten Individuen führen werden. Die Konsequenzen der marktlichen Anpassungsreaktionen an gegebene staatlich determinierte Institutionen stellen gleichzeitig einen wichtigen Bestimmungsfaktor für die Entfaltung politischer Einflußnahmeaktivitäten zur Änderung fiskalischer Institutionen dar. Gelingt den besteuerten Individuen durch die private Anpassung eine Verminderung der Steverzahlung, wird das für Distributionszwecke verfügbare Steueraufkommen geschmälert ${ }^{1)}$. Bei Gültigkeit der Budgetrestriktion muß entweder eine Einschränkung der Vergünstigungen oder eine Erhöhung der Besteuerung vorgenommen werden. Als Folge der erhöhten Belastung werden einige Wirtschaftssubjekte beginnen, politischen Einfluß auszuüben, während andere Individuen, die schon politisch aktiv waren, die Intensität ihrer Bemühungen im Hinblick auf die erfolgreiche Durchsetzung ihrer gruppenspezifischen Distributionsinteressen steigern werden ${ }^{2}$. In gleicher Weise werden die Begünstigungsempfänger den politischen Entscheidungsprozeß beeinflussen, um den drohenden Verlust ihrer Vorteile abzuwenden.
1) Vgl. Buchanan (1966), S. $35 \mathrm{ff}$.
2) Vgl. auch Breton (1974), S. $90 \mathrm{f}$. 


\subsection{Die Effekte politischer EinfluBnahmeaktivitäten}

Die politischen Aktivitäten der Gruppenmitglieder zur vorteilhaften Veränderung der fiskalischen Regelungen werden durch die gruppenspezifische Einflußnahmefunktion

(V.12) $\quad I^{s}=I^{s}\left(p^{s}, p^{t}\right)$

bzw.

(V.13)

$$
I^{t}=I^{t}\left(p^{t}, p^{s}\right)
$$

dargestellt, wobei $p^{s}$ und $p^{t}$ den politischen Druck der begünstigten bzw. besteverten Interessengruppe bezeichnen. Die Einbeziehung des politischen Drucks der Opposition als Argument der entsprechenden Funktion jeder Interessengruppe symbolisiert die Interdependenz der Rent-Seeking-Aktivitäten ${ }^{1}$. Es gilt:

$$
I_{s}^{s}=\frac{\partial I^{s}}{\partial p^{s}}>0 \quad b z w . \quad I_{t}^{s}=\frac{\partial I^{s}}{\partial p^{t}}<0
$$

und

$$
I_{t}^{t}=\frac{\partial I^{t}}{\partial p^{t}}>0 \quad \text { bzw. } \quad I_{s}^{t}=\frac{\partial I^{t}}{\partial p^{s}}<0 .
$$

Außerdem wird $I_{s s}^{s}\left\langle 0, I_{t t}^{t}\left\langle 0, I_{t t}^{s}>0\right.\right.$ und $I_{s s}^{t}>0$ unterstellt ${ }^{2}$ ).

1) Vgl. Becker (1983), S. 375 ff. und Laux (1984), S. 123.

2) Die Subskripte bezeichnen die Ableitung(en) nach dem jeweiligen Argument der entsprechenden Einflußnahmefunktion. 
Die Höhe des politischen Einflusses einer Gruppe ist danach negativ abhängig von der Menge des politischen Drucks, den die Opposition auf den politischen Entscheidungsprozeß ausübt.

Die zweite Ableitung der Funktion der politischen Einflußnahme der Vergünstigungsempfänger nach dem politischen Druck der Opposition wird gemäß

(V.16)

$$
\frac{\partial^{2} I^{s}}{\partial p^{s} \partial p^{t}}=\frac{\partial^{2} I^{s}}{\partial p^{t} \partial p^{s}}
$$

bzw.

$$
\text { (V.17) } \quad I_{\text {st }}^{s}=I_{\text {ts }}^{s} \gtreqless 0
$$

symbolisiert, die mutatis mutandis für (V.13) gilt.

Die Einflußnahme der Steverzahlergruppe

$$
I^{t}\left(p^{t}, p^{s}\right)=-n^{t} F\left(T r^{t}\right)
$$

zielt auf eine Verminderung der Steverbelastung ab, während die Begünstigungsempfänger ihren politischen Einfluß

$$
I^{s}\left(p^{s}, p^{t}\right)=n^{s} G\left(T r^{s}\right)
$$

auf eine Erhöhung des Subventionsvolumens richten, d.h. "the amount of monetary subsidy a group receives will equal the amount of positive political influence the group exerts"1).

1) Coe/Wilber (1985), S. 41. 
Die politische Budgetrestriktion (V.10) verbindet die beiden Funktionen der politischen Einflußnahme, so daß sich

(V.20) $\quad T=n^{t} F\left(T^{t}\right)=-I^{t} \equiv I^{s}=n^{s} G\left(T^{s}\right)=S$

ergibt. Diese Beziehung impliziert, daß mit einer steigenden Einflußnahme einer Interessengruppe ceteris paribus eine gruppenspezifisch begünstigende Änderung des Verteilungsresultats verbunden ist ${ }^{1}$. Aus diesem Grund ist die andere Gruppe gezwungen, sich am politischen Wettbewerb zu beteiligen, um Distributionsverluste abzuwenden. Die Effekte der politischen Einflußnahme induzieren eine Veränderung des Steuer- bzw. Subventionsvolumens und tangieren damit zwangsläufig die Interessen beider Gruppen.

Bei Gültigkeit der Budgetrestriktion muß allerdings eine Steigerung der Einflußnahme einer Gruppe mit einer gleich hohen Verminderung der Einflußnahme der Opposition einhergehen ${ }^{2}$. Aus diesem Zusammenhang resultiert für den aggregierten Einfluß

$$
I^{s}+I^{t}=0
$$

womit unter Bezug auf (V.14) und (V.15) auch

(V.22) $\quad \frac{\partial I^{s}}{\partial p^{s}} \equiv I_{s}^{s}=-I_{s}^{t} \equiv-\frac{\partial I^{t}}{\partial p^{s}}$

gilt. Aus dem Anstieg der gruppenspezifischen Einflußnahme der

1) Vgl. Becker (1983), S. $375 \mathrm{ff}$.

2) Vgl. Coe/Wilber (1985), S. 41. 
Begünstigungsempfänger durch gesteigerte Produktion von politischem Druck resultiert ein Verteilungsvorteil, indem der politische Einfluß der Steverzahlergruppe in gleichem Ausmaß reduziert wird. Analog ergibt sich bei Erhöhung des Drucks der Steuerzahler eine Verminderung der Steuerbelastung, da ceteris paribus der Einfluß der Vergünstigungsgruppe abnimmt.

Die Beziehung zwischen dem von beiden Gruppen produzierten politischen Druck und dem resultierenden politischen Einfluß für die Gruppe der Begünstigungsempfänger wird in Abbildung 9 illustriert.

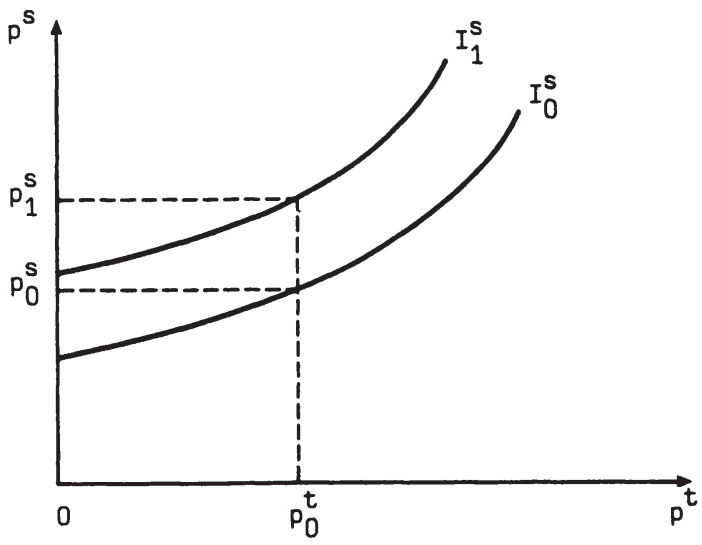

Abb. 9: Die gruppenspezifische Einflußnahme

Dabei ist auf der Abszisse die Menge des politischen Drucks der Steuerzahlergruppe $\left(p^{t}\right)$ abgetragen, während die entsprechenden Mengeneinheiten für die Begünstigten ( $p^{s}$ ) auf der Ordinate abgelesen werden können. 
Da die Höhe des politischen Einflusses mit zunehmendem Druck der Begünstigten zunimmt, dagegen mit steigendem Druck der Opposition sinkt, kann eine "Kurve gleichen Einflusses" in der Gestalt von $I^{S}$ eingezeichnet werden. Diese Funktion beschreibt alle Kombinationen von $p^{s}$ und $p^{t}$, die das gleiche Einflußnahmeniveau für die Begünstigten implizieren ${ }^{1)}$. Bei gegebener Höhe des politischen Drucks seitens der Steverzahlergruppe ( $p_{0}^{t}$ ) ist das Einflußnahmeniveau - und damit das Ausmaß des Subventionsvolumens bzw. der Steuerbelastung - um so höher, je mehr politischer Druck von den Begünstigten ( $p_{0}^{s} b z w . p_{1}^{s}$ ) ausgeübt wird. Damit weist $I_{1}^{S}$ ein höheres Ausmaß an politischem Einfluß als $I_{0}^{s} a u f^{2)}$.

Als Konsequenz dieser Überlegungen ist festzuhalten, daß der Wettbewerb zwischen Interessengruppen um politischen Einfluß als distributives Nullsummenspiel und als allokatives Negativsummenspiel aufgrund der Mehrbelastungseffekte durch private Anpassungsreaktionen interpretiert werden kann. Für jede Gruppe besteht die Möglichkeit, durch Steigerung ihres politischen Einflusses eine gruppenspezifisch begünstigende Änderung des Verteilungsresultats anzustreben. Daher werden die Gruppen "compete for political influence by spending time, energy, and money on the production of political pressure" ${ }^{3}$ ). In diesem Wettbewerb müssen allerdings auch die Auswirkungen der gruppeninternen Interessenkonstellation auf die Produktion politischen Drucks berücksichtigt werden.

1) Vgl. Findlay/Wellisz (1983), S. 472 f. bzw. auch Balisacan/ Roumasset (1987), S. $243 \mathrm{f}$.

2) Becker (1983), S. 387 .

3) Becker (1983), S. 377. 
Die Produktionsfunktion politischen Drucks für jede Gruppe werde durch
(V.23)
$p^{s}=p^{s}\left(m^{s}, n^{s}\right)$
bzw.
$p^{t}=p^{t}\left(m^{t}, n^{t}\right)$

dargestellt, wobei

(v.24) $\quad m^{s}=R^{s} n^{s}$ bzw. $m^{t}=R^{t} n^{t}$

gilt. Dabei symbolisiert $\mathrm{m}^{s}\left(\mathrm{~m}^{t}\right)$ die gesamten für politische Distributionsvorhaben zur Verfügung stehenden Ressourcen der Begünstigten (Steverzahler), während der individuelle Ressourcenbeitrag eines repräsentativen Gruppenmitgliedes mit $R^{S}\left(R^{t}\right)$ bezeichnet wird.

Die Produktion zusätzlichen politischen Drucks wird gemäß

(v.25) $\quad p_{m}^{i}=\frac{\partial p^{i}}{\partial m^{i}}>0 \quad$ für $i=s, t$

mit höherer Ressourcenausstattung ansteigen. Unterstellt man konstante individuelle Ressourcenbeiträge, ist dazu die Gewinnung weiterer Mitglieder erforderlich. Damit wird allerdings das aus der Kollektivguteigenschaft ${ }^{1)}$ der Distributionsvorteile resultierende "Trittbrettfahrer"-Verhalten der Gruppenmitglieder relevant, das sich negativ auf die Produktion politischen Drucks auswirkt ${ }^{2}$.

1) Da die Vorteile der politischen Aktivitäten jeweils allen (identischen) Gruppenmitgliedern zugute kommen, "subsidies and taxes would be public goods". Becker (1985), S. 332.

2) Vgl. insbesondere Pethig (1987), S. $153 \mathrm{ff}$. 
Der Gesamteffekt einer steigenden Gruppengröße auf die Grenzproduktivität eines höheren Ressourceneinsatzes für politische Aktivitäten kann in Form von

$$
d\left(\frac{\partial p^{i}}{\partial m^{i}}\right)=\frac{\partial}{\partial m^{i}}\left(\frac{\partial p^{i}}{\partial m^{i}}\right) d m^{i}+\frac{\partial}{\partial n^{i}}\left(\frac{\partial p^{i}}{\partial m^{i}}\right) d n^{i}
$$

ermittelt werden, woraus nach Umformung und Substitution

$$
\frac{\partial p_{m}^{i}}{\partial n^{i}}=\frac{\partial^{2} p^{i}}{\partial m^{i} \partial n^{i}}=R^{i} p_{m m}^{i}+p_{m n}^{i}
$$

folgt ${ }^{1)}$. Das Vorzeichen des ersten Summanden auf der rechten Seite dieser Gleichung hängt davon ab, ob abnehmende oder steigende Skalenerträge beim Ressourceneinsatz für die Produktion politischer Einflußnahme vorliegen. Der zweite Summand ist Ausdruck der gruppeninternen Interessenkonstellation und besitzt aufgrund der "Trittbrettfahrer"-Problematik ein negatives Vorzeichen.

1) "Therefore, total expenditures on the production of pressure equals the sum on direct political activity and on the control of free riding." Becker (1983), S. 377. 


\section{Das Gleichgewicht der Distributionsinteressen}

Auf der Grundlage dieser Modellstruktur kann ein Gleichgewicht der politischen Distributionsinteressen abgeleitet werden. Beide Gruppen streben nach Verteilungsvorteilen, indem sie Ressourcen für die Produktion von politischem Druck aufwenden. Dabei wird vereinfachend ein Cournot-Nash-Anpassungsprozeß unterstellt, d.h. jede der beiden Gruppen verhält sich autonom und bestimmt ihren Ressourceneinsatz für politische Einflußnahme auf der Grundlage eines gegebenen Rent-SeekingEinsatzes der Opposition ${ }^{1}$.

Die Zielfunktion eines repräsentativen Individuums der Vergünstigungs- bzw. Steverzahlergruppe läßt sich daher als

$$
Y_{1}^{s}=Y_{0}^{s}+T r^{s}-R^{s} \rightarrow \max !
$$

bzw.

$$
Y_{1}^{t}=Y_{0}^{t}-T^{t}-R^{t} \rightarrow \max !
$$

darstellen, d.h. ein Gruppenmitglied "maximizes his utility by spending resources on political activities to create pressure that affects his subsidies or taxes" 2 ).

1) Vgl. Findlay/Wellisz (1983), S. 472 bzw. Becker (1983), S. 377 .

2) Becker (1985), S. 330 . 
Die notwendigen Bedingungen werden als

(V.30) $\quad \frac{d Y_{1}^{s}}{d R^{s}}=\frac{d T r^{s}}{d R^{s}}-1=0$

bzw.

(V.31) $\quad \frac{d Y_{1}^{t}}{d R^{t}}=-\frac{d T r^{t}}{d R^{t}}-1=0$

ermittelt, woraus unmittelbar

(v.32) $\frac{d r^{s}}{d R^{s}}=1 \quad$ bzw. $\quad \frac{d r^{t}}{d R^{t}}=-1$

folgt. D.h. der marginale Rent-Seeking-Ressourceneinsatz des begünstigten bzw. benachteiligten Individuums muß im Optimum dem marginalen Distributionsgewinn bzw. -verlust entsprechen. Berücksichtigt man (V.18) bzw. (V.19), können die Gleichgewichtsbedingungen in Form von

$$
\frac{d T^{s}}{d R^{s}}=\frac{1}{n^{s} G^{\prime}} \cdot \frac{\partial I^{s}}{\partial p^{s}} \cdot \frac{\partial p^{s}}{\partial m^{s}} \cdot \frac{\partial m}{\partial R^{s}}=\frac{I_{s}^{s} p_{m}^{s}}{G^{\prime}}=1
$$

bzw.

(v.34) $\frac{d T r^{t}}{d R^{t}}=-\frac{1}{n^{t} F^{\prime}} \cdot \frac{\partial I^{t}}{\partial p^{t}} \cdot \frac{\partial p^{t}}{\partial m^{t}} \cdot \frac{\partial m^{t}}{\partial R^{t}}=\frac{I_{t}^{s} p_{m}^{t}}{F^{\prime}}=-1$

bestimmt werden, wobei 
(V.35) $\quad I_{t}^{t}=-I_{t}^{s}$

substituiert wurde. Diese Form der Darstellung verdeutlicht, daß neben der Effizienz bei der Produktion politischen Drucks insbesondere das Ausmaß der gruppenspezifischen Mehrbelastung ein wichtiger Bestimmungsfaktor des politischen Gleichgewichts ist.

Die hinreichenden Bedingungen werden durch

$$
\frac{d^{2} T r^{s}}{d R^{s^{2}}}=\frac{\left(I_{s s}^{s} p_{m}^{s^{2}}+I_{s}^{s} p_{m m}^{s}\right) n^{s}}{G^{\prime}}-\frac{I_{s}^{s} p_{m}^{s} G^{\prime \prime}}{G^{\prime 2}}<0
$$

bzw.

(V.37) $\quad \frac{d^{2} T r^{t}}{d R^{t^{2}}}=\frac{\left(I_{t t}^{s} p_{m}^{t^{2}}+I_{t}^{s} p_{m m}^{t}\right) n^{t}}{F^{\prime}}+\frac{I_{t}^{s} p_{m}^{t} F^{\prime \prime}}{F^{\prime 2}}>0$

gegeben. Daraus folgt, daß neben abnehmenden Zuwächsen politischer Einflußnahme ( $I_{s s}^{s}<0, I_{t t}^{s}>0$ ) und steigenden Mehrbelastungskosten bei beiden Gruppen ( $G ">0, F^{\prime \prime}<0$ ) abnehmende Skalenerträge bei der Produktion politischen Drucks $\left(p_{m m}^{i} \leq 0\right)$ der Interessengruppen erforderlich sind ${ }^{1)}$.

1) Vgl. dazu Becker (1985), S. 378 . 
Auf der Grundlage dieser Beziehungen kann ein Gleichgewicht der Distributionsinteressen ermittelt werden, das neben dem politischen Druck beider Gruppen auch das Ausmaß der Verteilungsgewinne bzw. -verluste bestimmt. Zu diesem Zweck wird davon ausgegangen, daß

$$
I_{s t}^{s}=\frac{\partial I^{s}}{\partial p^{s} \partial p^{t}}>0 \quad \text { bzw. } \quad I_{t s}^{t}=\frac{\partial I^{t}}{\partial p^{t} \partial p^{s}}<0
$$

gilt, d.h. es wird eine Komplementaritätsbeziehung in der Einflußnahmefunktion der Begünstigten zwischen $p^{s}$ und $p^{t}$ unterstellt. Diese Annahme impliziert einen Anstieg des politischen Drucks $p^{s}$ bei einer Erhöhung von $p^{t}$, da die Grenzproduktivität von $p^{s}$ im Hinblick auf die gruppenspezifische Einflußnahme $I^{S}$ zunimmt. Allerdings resultiert bei einer Steigerung von $p^{s}$ durch die Begünstigten eine Verminderung von $\mathrm{p}^{\mathrm{t}}$, da der negative Effekt auf $I^{s}$ reduziert wird.

Zur Ermittlung der Reaktionsfunktionen beider Gruppen bei unabhängigem Anpassungsverhalten werden die Ableitungen

$$
\frac{d^{2} T r^{s}}{d R^{s} d R^{t}}=\frac{I_{s t}^{s} p_{m}^{s}}{G^{\prime}}+\frac{I_{s}^{s} P_{m}^{s} F^{\prime} G^{\prime \prime}}{G^{\prime}{ }^{3} P_{m}^{t} n^{s}}>0 \text { für } I_{s t}^{s} \geqq 0
$$

bzw.

$$
\text { (v.40) } \quad \frac{d^{2} T r^{t}}{d R^{t} d R^{s}}=\frac{I_{t s}^{t} p_{m}^{t}}{F^{\prime}}-\frac{I_{t}^{t} p_{m}^{t} G^{\prime} F^{\prime \prime}}{F^{\prime}{ }^{3} p_{m}^{s} n^{t}}<0 \quad \text { für } I_{t s}^{t} \leqq 0
$$

betrachtet. 
Daraus folgt, daß eine steigende Mehrbelastung ( $G ">0$, $F^{\prime \prime}(0)$ die Zunahme des optimalen Drucks der Steuerzahler bzw. der Begünstigungsempfänger induziert, wenn eine der Gruppen ihren politischen Druck steigert ${ }^{1}$.

Auf der Grundlage dieser Beziehungen lassen sich die Reaktionsfunktionen der beiden Gruppen ableiten. Aus Gründen der Vereinfachung werden zu diesem Zweck die partiellen bzw. gemischten Ableitungen ( $V .36, V .37$ bzw. V.39, V.40) in Form von $f_{i j}(i=s, t ; j=s, t)$ dargestellt, wobei für ein stabiles Gleichgewicht

$$
\frac{f_{t s}}{f_{t t}}>\frac{f_{s t}}{f_{s s}}
$$

gelten muß. D.h. die Steigung der Reaktionsfunktion der Steuerzahlergruppe muß größer als die Steigung der Reaktionsfunktion der Gruppe der Vergünstigungsempfänger sein. Aufgrund der Tatsache, daß eine steigende Mehrbelastung durch Steuern und Vergünstigungen zu einem Anstieg des gleichgewichtigen politischen Drucks bei einer Erhöhung der Druckausübung der Opposition führt, besitzen die Reaktionsfunktionen eine positive Steigung ${ }^{2}$. Bedingung $(V .41)$ ist insbesondere dann erfüllt,

1) Vgl. Becker (1983), S. 378 .

2) "In general, the pressure exerted by one group will increase when the pressure by an opposing group is raised, primarily as a result of increasing deadweight losses as taxes and subsidies are increased." Coe/Wilber (1985). S. 42 . 
wenn keine wechselseitigen Beziehungen bei der Einflußnahmeproduktion existieren ( $I_{s t}=I_{t s}=0$ ) und keine Mehrbelastungen $\left(F^{\prime \prime}=0, G^{\prime \prime}=0\right)$ bei beiden Distributionsparteien auftreten $^{1)}$. Zur Illustration der Zusammenhänge kann Abbildung 10 dienen, in der die Reaktionsfunktion der Gruppe der Steuerzahler (Vergünstigungsempfänger) mit $t_{0} t_{O}\left(s_{0} s_{0}\right)$ bezeichnet wird.

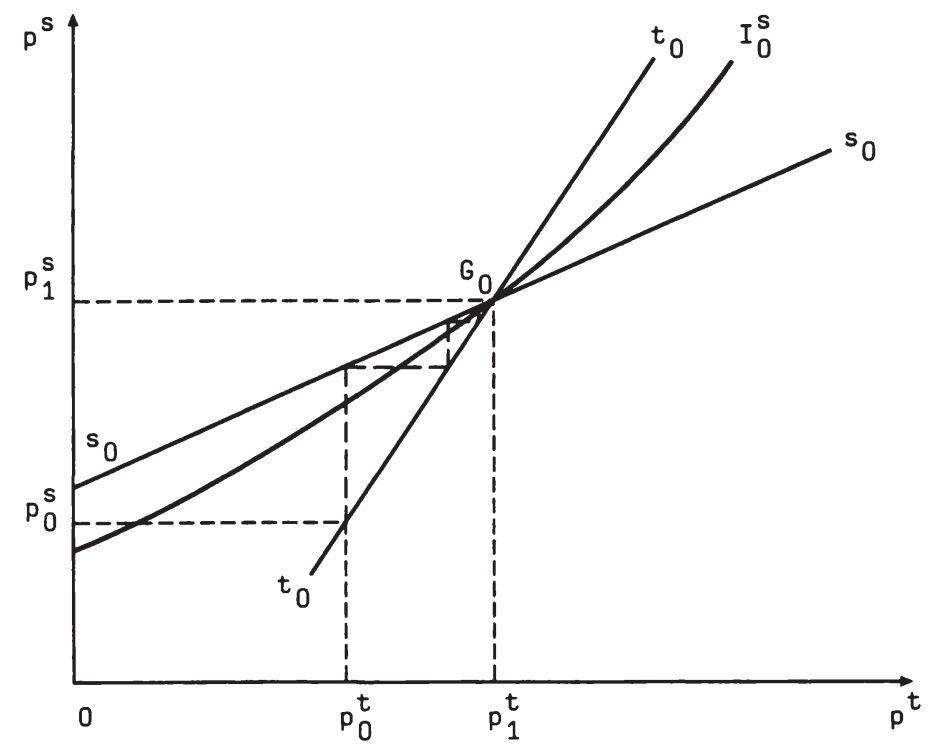

Abb. 10: Das Gleichgewicht der Distributionsinteressen

1) In diesem Fall wird in Abbildung 10 die Reaktionskurve soso durch eine Parallele zur Abszisse wiedergegeben, während die Reaktionskurve toto durch eine Vertikale bei beliebigem $\mathrm{p}^{\mathrm{s}}$ beschrieben werden kann. Vgl. dazu auch Findlay/Wellisz $(1983)$, S. 472 f. 
Als Ausgangspunkt sei angenommen, daß die Steuerzahler politischen Druck in Höhe von $\mathrm{p}_{0}^{t}$ entfalten, um eine Reduktion ihrer Belastung zu erreichen. Die Opposition reagiert, indem sie entsprechend ihrer Reaktionsfunktion selbst den Ressourceneinsatz zur Beeinflussung des politischen Prozesses steigert. Die Steuerzahler werden durch dieses Verhalten veranlaßt, ihrerseits die Einflußnahmeaktivitäten wiederum zu erhöhen. Ein stabiles Gleichgewicht ergibt sich in $G_{0}$, bei dem keine Gruppe mehr eine Veränderung ihrer politischen Aktivitäten vornimmt. Dieses Gleichgewicht bestimmt die von beiden Gruppen für Einflußnahmeaktivitäten aufgewendeten Ressourcen ( $\left.p_{1}^{t}, p_{1}^{s}\right)$ sowie das Einflußnahmeniveau $I_{0}^{S}$ und damit die gruppenspezifischen Verteilungsergebnisse.

Zur Analyse der Veränderung der Gleichgewichtswerte des politischen Drucks beider Interessengruppen wird die Methode der komparativen Statik ${ }^{1}$ verwendet. Dabei werden alternative Gleichgewichtszustände verglichen, wobei der Anpassungsprozeß zum neven Gleichgewicht nicht untersucht wird ${ }^{2)}$. Die Abweichung $z$ wischen dem bisherigen und dem neven Gleichgewicht wird auf die Wirkungen von Parametervariationen zurückgeführt. Zu diesem Zweck wird ein Gleichungssystem in impliziter Form

$$
f_{s s} \frac{d p^{s}}{d \lambda}+f_{s t} \frac{d p^{t}}{d \lambda}+s_{\lambda}=0
$$

$$
f_{t s} \frac{d p^{s}}{d \lambda}+f_{t t} \frac{d p^{t}}{d \lambda}+t_{\lambda}=0
$$

1) Eine zusammenfassende Darstellung der grundsätzlichen Vorgehensweise bei der Methode der komparativen Statik gibt Kuska (1973), S. $119 \mathrm{ff}$.

2) Vgl. Samuelson (1963), S. 8. 
formuliert ${ }^{1)}$, wobei $s_{\lambda}$ bzw. $t_{\lambda}$ die unmittelbaren Konsequenzen der Variation eines modellexogenen Parameters $\lambda$ für die gruppenspezifische Gleichgewichtsbedingung ( $V .33$ bzw. V.34) symbolisiert. Zur Vereinfachung der weiteren Überlegungen wird das System in Matrixform

(V.43)

$$
\left[\begin{array}{ll}
f_{s s} & f_{s t} \\
f_{t s} & f_{t t}
\end{array}\right]\left[\begin{array}{l}
\frac{d p^{s}}{d \lambda} \\
\frac{d p^{t}}{d \lambda}
\end{array}\right]=\left[\begin{array}{l}
-s_{\lambda} \\
-t_{\lambda}
\end{array}\right]
$$

dargestellt. Unter Verwendung der Cramer'schen Regel ${ }^{2}$ ) wird

(v.44) $\frac{d p^{s}}{d \lambda}=\frac{-s_{\lambda} f_{t t}+t_{\lambda} f_{s t}}{|F|}$

und

(v.45) $\frac{d p^{t}}{d \lambda}=\frac{s_{\lambda} f_{t s}-t_{\lambda} f_{s s}}{|F|}$

1) Vgl. Samuelson (1963), S. $30 \mathrm{ff}$. und Becker (1983), S. 397.

2) Zur Anwendung der Cramer'schen Regel bei der Lösung linearer Gleichungssysteme vgl. Kuska (1973), S. 46. 
ermittelt, wobei für die Determinante

(v.46) $\quad|F|=f_{s s} \cdot f_{t t}-f_{s t} \cdot f_{t s}>0$

$\operatorname{gilt}{ }^{1)}$

Im weiteren werden lediglich die Effekte auf eine der beiden Gleichgewichtsbedingungen betrachtet, so daß entweder $s_{\lambda}$ oder $t_{\lambda}$ gleich null ist. Damit wird die Richtungsänderung des gleichgewichtigen politischen Drucks einer Gruppe infolge einer exogenen Parametervariation anhand des Vorzeichens von

$$
\operatorname{sgn} \frac{d p^{s}}{d \lambda^{s}}=\operatorname{sgn} s_{\lambda}=\operatorname{sgn} \frac{d p^{t}}{d \lambda^{s}} \quad \text { für } f_{t s}<0
$$

bzw.

(V.48)

$$
\operatorname{sgn} \frac{d p^{t}}{d \lambda^{t}}=\operatorname{sgn} t_{\lambda}=\operatorname{sgn} \frac{d p^{s}}{d \lambda^{t}} \quad \text { für } f_{s t}>0
$$

bestimmt. Auf dieser Grundlage können die Wirkungen von Veränderungen einzelner Bestimmungsfaktoren auf die Gleichgewichtswerte des politischen Einflusses beider Gruppen untersucht werden.

1) Aufgrund von (V.46) ist die Matrix (V.43) regulär. 


\section{Die Effekte einer Veränderung der Determinanten des Distributionsgleichgewichts}

Bei der Analyse der Effekte von Parametervariationen auf das politische Gleichgewicht wird im folgenden auf die Konsequenzen einer effizienteren Produktion politischen Drucks, einer veränderten Mehrbelastung staatlicher Maßnahmen sowie einer steigenden Gruppengröße für die Gleichgewichtsstruktur von Stevern und Vergünstigungen eingegangen.

\subsection{Steigerung der Produktionseffizienz politischen Drucks}

Ausgangspunkt der Überlegungen ist die Vorstellung, daß der Gruppe der Vergünstigungsempfänger eine - gegenüber der bisherigen Situation - wirksamere Kontrolle des "Trittbrettfahrer"-Verhaltens ihrer Mitglieder gelingt ${ }^{1)}$. Aus der damit verbundenen Verminderung der Organisationskosten resultiert aufgrund von ( $V .27$ ) eine Steigerung der Grenzproduktivität zusätzlichen Ressourceneinsatzes für politische Aktivitäten. Damit ergibt sich gemäß

$$
s_{\lambda}=\frac{I_{s}^{s} p_{m \lambda}^{s}}{G^{\prime}}>0 \text { für } p_{m \lambda}^{s}>0
$$

ein Anstieg des politischen Drucks dieser Gruppe, d.h. jedem gegebenen Druck $p^{t}$ der Opposition entspricht ein erhöhter Gleichgewichtswert $p^{s}$ der Vergünstigungsgruppe.

1) Die Argumentation gilt in gleicher Weise für alle gruppeninternen Aktivitäten, die zu einer effizienteren Produktion politischen Drucks führen. So kann beispielsweise auch die Produktionstechnik verbessert werden. 
Dieser Zusammenhang wird in Abbildung 11 durch die Verschiebung der Reaktionsfunktion von $s_{0} s_{0}$ auf $s_{1} s_{1}$ zum Ausdruck gebracht ${ }^{1)}$.

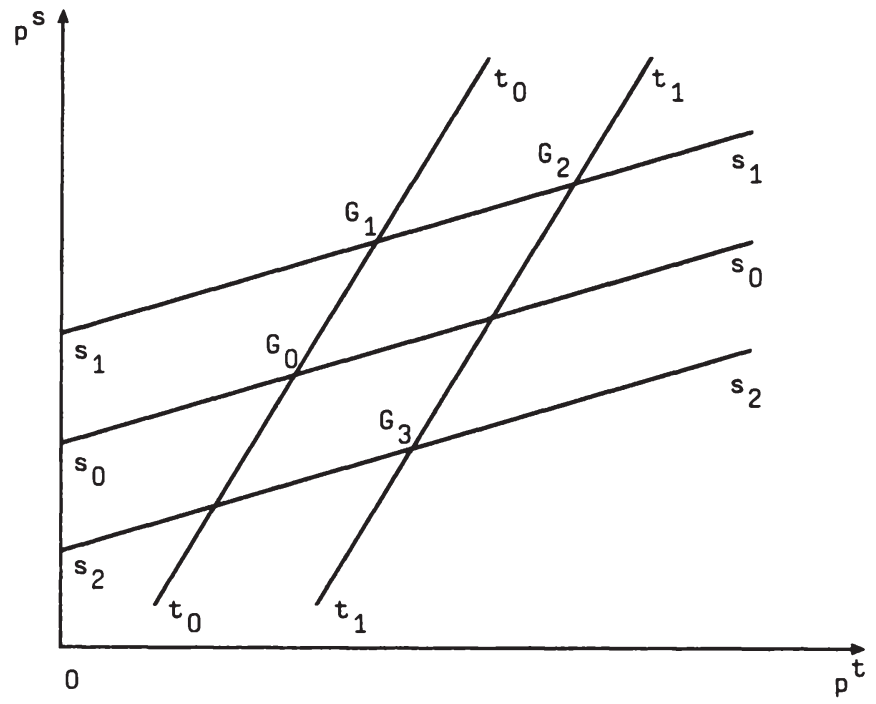

Abb. 11: Die Effekte von Parametervariationen auf das Distributionsgleichgewicht

Die Bewegung von $G_{0}$ zum neuen Gleichgewichtspunkt $G_{1}$ impliziert, daß auch der von der Steverzahlergruppe produzierte politische Druck steigt. Unabhängig von der induzierten Wirkung auf $p^{t}$ ergibt sich eine Erhöhung der Vergünstigungen bzw. der Steuerbelastung, da mit der effizienteren Produktion politischen Drucks der Einfluß der Begünstigten zunimmt.

1) Vgl. Becker (1983), S. 379 . 
In analoger Weise kann für die Steuerzahlergruppe

(V.50) $\quad t_{\lambda}=\frac{I_{t}^{S} p_{m \lambda}^{t}}{F^{\prime}}>0$ für $p_{m \lambda}^{t}>0$

ermittelt werden, d.h. eine effizientere Produktion politischen Drucks der Steuerzahler schlägt sich ceteris paribus in einer verminderten Steuerbelastung nieder. Daher verschiebt sich die Reaktionsfunktion der Steuerzahlergruppe von $t_{0} t_{0}$ auf $t_{1} t_{1}$ (Abbildung 11). Aus diesen überlegungen folgt, "that a group which becomes more efficient at producing political pressure will be able to reduce its taxes or raise its subsidy"1).

Ergibt sich eine Steigerung der Produktionseffizienz bei beiden Gruppen, stellt sich das neve Gleichgewicht in $G_{2}$ ein. Der Einfluß und damit das Distributionsresultat müssen sich nicht verändern, da sich die Wirkungen des zusätzlichen politischen Drucks im Hinblick auf die gruppenspezifische Einflußnahme gegenseitig kompensieren können. Ein Anstieg des Einflusses beider Interessengruppen ist aufgrund der politischen Budgetrestriktion (V.21) ausgeschlossen. Aus diesem Grund wird die Effektivität bzw. Schlagkraft einer Interessengruppe im Distributionskonflikt von der - zur konkurrierenden Gruppe - komparativ effizienteren Produktion politischen Drucks bestimmt.

Beispielsweise kann die Vergünstigungsgruppe hohe Vorteile erzielen, obwohl die überwindung des "Trittbrettfahrer"-Verhaltens der Mitglieder mit hohen Kosten verbunden ist, sofern sich die Steuerzahlergruppe noch größeren Organisationsproblemen gegenübersieht. Mit anderen Worten ist weniger die (absolute)

1) Coe/Wilber (1985), S. 42 f. 
gruppeninterne Produktionseffizienz als vielmehr die (relativ) zur Opposition effizientere Produktion politischen Drucks für die Realisierung von Distributionsvorteilen entscheidend ${ }^{1}$.

Da in kleineren Gruppen bei niedriger Ressourcenausstattung "economies of scale" bei der Produktion politischen Einflusses vorliegen und das "Trittbrettfahrer"-Phänomen nicht ins Gewicht fällt, wird ein Anstieg der Mitgliederzahl die Produktion politischen Drucks positiv beeinflussen. Diese Folgerung ergibt sich aus der Tatsache, daß die Nutzen steigender Skalenerträge des Ressourceneinsatzes die Kosten der Kontrolle des "Trittbrettfahrer"-Verhaltens überwiegen werden. Mit steigender Mitgliederzahl kann sich eine Umkehrung dieser Entwicklung ergeben, da zunehmende Organisationskosten abnehmenden Skalenerträgen gegenüberstehen.

Allerdings ist mit einem Anstieg der Gruppengröße eine Verminderung der marginalen Mehrbelastungskosten für beide Gruppen verbunden, da bei gegebenem Subventions-bzw. Steuervolumen die Vergünstigung bzw. Steuerbelastung für jedes Gruppenmitglied reduziert wird. Damit ergibt sich die Notwendigkeit, die Wirkungen einer veränderten Mehrbelastung auf die Entfaltung politischer Einflußnahmeaktivitäten zu analysieren.

1) "The political effectiveness of a group is mainly determined not by its absolute efficiency ... but by its efficiency relative to the efficiency of other groups." Becker (1983), S. 380. Vgl. auch Pommerehne/Kirchgässner (1988), S. 229 . 


\subsection{Veränderung der Mehrbelastung}

Die mit staatlichen Umverteilungsaktivitäten verbundene Mehrbelastung stellt einen wesentlichen Bestimmungsfaktor für die Höhe des gruppenspezifischen politischen Drucks dar. Während sich eine Erhöhung (Senkung) der marginalen Mehrbelastung auf seiten der Steverzahler in einer Verminderung (Steigerung) von $F^{\prime}$ niederschlägt, impliziert ein marginal zunehmender (abnehmender) "excess burden" bei den Vergünstigungsempfängern eine Erhöhung (Senkung) von G,1).

Eine exogen determinierte Verminderung der marginalen Mehrbelastung der Begünstigten führt gemäß

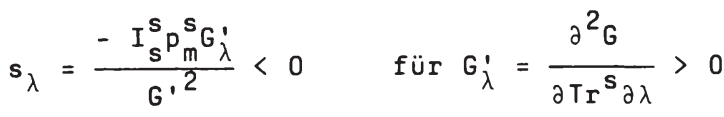

zu einem Anstieg des politischen Drucks dieser Gruppe, weil eine gegebene Steigerung der Vergünstigungen eine im Vergleich dazu geringere Zunahme des Steueraufkommens erfordert.

Andererseits resultiert aus einer Reduktion der Zusatzlast der Besteverung aufgrund von

$$
-t_{\lambda}=\frac{I_{t}^{s} p_{m}^{t} F_{\lambda}}{F^{\prime 2}}<0 \text { für } F_{\lambda}^{\prime}=\frac{\partial^{2} F}{\partial T^{t} \partial \lambda}>0
$$

eine sinkende Einflußnahmeaktivität der Steuerzahlergruppe, da eine gegebene Verminderung der Steverlast einen größeren negativen Effekt im Hinblick auf eine Senkung des Steueraufkommens hervorruft.

1) Vgl. Becker (1985), S. $333 \mathrm{f}$. 
In analoger Weise ergibt sich, daß ein Anstieg der Mehrbelastung den politischen Druck der Steuerzahlergruppe erhöht, während eine marginale Zunahme der Zusatzlast für die Begünstigten zu einer Verminderung der politischen Aktivitäten führt.

Diese Argumentation soll an folgendem Beispiel erläutert werden: Angenommen die Erhebung eines zusätzlichen Steueraufkommens in Höhe von einer Geldeinheit (GE) führt aufgrund der Mehrbelastung $z u$ einer wesentlich höheren effektiven Nutzeneinbuße bei den Steverzahlern, die in Form eines monetären Äquivalents in Höhe von $10 \mathrm{GE}$ bemessen werden kann. In dieser Konstellation ist die Produktion zusätzlichen politischen Drucks lohnend, da mit einer Reduktion des Steueraufkommens ein hoher Nutzenzuwachs bzw. Verteilungsvorteil verbunden ist. Wird andererseits mit dem zusätzlichen Stever-bzw. Subventionsvolumen von einer Geldeinheit infolge einer hohen Mehrbelastung auf seiten der Begünstigten lediglich eine geringe effektive Nutzensteigerung (z.B. 0,1 GE) induziert, so sind die Anreize zur weiteren Produktion politischen Drucks entsprechend niedriger ${ }^{1)}$.

Die Konsequenzen eines exogenen Anstiegs der Mehrbelastung bei beiden Distributionsparteien lassen sich in Abbildung 11 durch die Verschiebung der gruppenspezifischen Reaktionsfunktion von $t_{0} t_{0}\left(s_{0} s_{0}\right)$ auf $t_{1} t_{1}\left(s_{2} s_{2}\right)$ verdeutlichen, die zu einem neven Gleichgewichtspunkt $G_{3}$ führt. Zur Realisierung des Gleichgewichts muß der politische Druck der Steuerzahlergruppe steigen oder der Druck der Begünstigten sinken. Damit resultiert die Schlußfolgerung, daß ein Anstieg der Mehrbelastung die gleichgewichtige Höhe der Vergünstigungen reduziert ${ }^{2}$.
1) Vgl. Coe/Wilber (1985), S. 43.
2) Vgl. Becker (1983), S. 381 . 
Aufgrund der Tatsache, daß mit zunehmenden Verzerrungen der privaten Allokationsentscheidungen der politische Druck der Belasteten (Begünstigten) zunimmt (abnimmt), hat die Steuerzahlergruppe ceteris paribus "an 'intrinsic' advantage in influencing political outcomes"1). Dieser Zusammenhang wird deutlich, wenn die Beziehungen (V.33) und (V.34) in Form von

(V.53)

$$
\frac{\frac{d T r^{s}}{d R^{s}}}{\frac{d T r^{t}}{d R^{t}}}=-\frac{I_{s}^{s} p_{m}^{s}}{I_{t}^{s} p_{m}^{t}} \cdot \frac{F^{\prime}}{G^{\prime}}
$$

dargestellt werden. Unterstellt man gleiche Mitgliederzahlen bzw. die gleiche Effizienz bei der Produktion politischen Drucks, werden die Effekte der Mehrbelastung durch

(V.54) $\frac{\frac{d T r^{s}}{d R^{s}}}{\frac{d T r^{t}}{d R^{t}}}=\frac{F^{\prime}}{G^{\prime}} \quad$ für $p^{s}=p^{t}$

bestimmt. Der Vorteil der Steverzahler ${ }^{2}$ nimmt mit steigender Mehrbelastung durch Steuern und Vergünstigungen zu, d.h. Wenn $F^{\prime}$ fällt und $G^{\prime}$ steigt. Lediglich im allokationsneutralen Fall $\left(F^{\prime}=G^{\prime}=1\right)$ resultieren keine Konsequenzen für die Höhe der politischen Einflußnahme beider Gruppen.

1) Becker (1983), S. 381 .

2) "Subsidized groups can overcome their intrinsic disadvantage with an optimal size, efficiency at producing pressure, success at converting pressure into influence, or with characteristics that raise their influence." Becker (1983), S. 382 . 
In gleicher Weise bewirkt die Mehrbelastung im Interessenwettbewerb eine erhöhte Einflußnahme der Vergünstigungsgruppe, wenn effizienzsteigernde Maßnahmen durchgeführt werden. Bei Gültigkeit der politischen Budgetgleichung (V.10) kann eine Verbesserung der Effizienz statlicher Umverteilung durch

$$
n^{s} r^{s}>n^{t} T_{r}^{t}
$$

dargestellt werden, d.h. die effektiven Vorteile der Begünstigten übersteigen die tatsächlichen Distributionsverluste der Steverzahler. Bei Marginalbetrachtung ist

$$
\text { (V.56) } \quad G^{\prime} n^{s} d T r^{s}=F^{\prime} n^{t} d T^{t} \text {, }
$$

so daß

(V.57) $\quad n^{s} d r^{s}>n^{t} d T r^{t}$

genau dann erfüllt ist, wenn

(V.58) $\quad G^{\prime}<F^{\prime}$

gilt.

In dieser Konstellation haben die Begünstigten gemäß (V.55) einen Anreiz, zusätzlichen politischen Druck zur Realisierung des Distributionsvorteils zu erzeugen. Aus diesem Grund werden sich im politischen Wettbewerb effizienzsteigernde Maßnahmen durchsetzen ${ }^{1)}$, es sei denn, die belastete Gruppe kann ihren komparativen Nachteil durch eine effizientere Produktion politischen Drucks ausgleichen.

1) "Political policies that raise efficiency are more likely to be adopted than policies that lower efficiency." Becker (1983), S. 384 . 
Unter Mehrbelastungsaspekten kann auch der Frage nachgegangen werden, welche Formen von Steuer-bzw. Subventionsinstitutionen aus dem Wettbewerb um politischen Einfluß hervorgehen. Zur Vereinfachung wird unterstellt, daß eine bestimmte Steuerbzw. Subventionsregelung die Bedingung

(V.59) $\quad F^{*}>F \quad$ bzw. $\quad G^{*}<G$

erfüllt. Diese Institutionen erzeugen eine geringere Mehrbelastung als alle anderen vergleichbaren Transferregelungen, die mit einem gegebenen Transfervolumen verbunden sind.

Unter der Annahme, daß die alternativ zur Verfügung stehenden Steuerinstitutionen keinen Einfluß auf die Höhe des Steueraufkommens besitzen, "a more efficient form of taxation will impose less of a burden on the taxed group and thus will be favored by them"1). Aufgrund der verminderten Belastung der Steuerzahler erfolgt eine Reduktion ihrer Produktion politischen Drucks, die ceteris paribus zu einem Anstieg der Vergünstigung führt. Bei gegebener Subventionsregelung ergibt sich neben der Erhöhung des Transfervolumens auch eine Steigerung des "Full Income" der Steuerzahler infolge des verminderten Ressourceneinsatzes für politische Einflußnahme. Daher werden beide Gruppen die effizientere Institution bevorzugen, d.h. "competition among pressure groups favors efficient methods of taxation"2).

1) Coe/Wilber (1985), S. 44.

2) Becker (1983), S. 386. Dieses Argumentationsmuster kann ebenfalls auf die Wahl alternativer Subventionsregelungen angewendet werden. 
Betrachtet man eine Konstellation ${ }^{1)}$, in der beide Gruppen sowohl bestevert als auch subventioniert werden, folgt als Ergebnis, daß ein Mitglied durch eine gleich hohe Reduktion von Steuern und Vergünstigungen seine ökonomische Position aufrechterhalten kann, "while aggregate output would be increased by the saving in deadweight costs from reduced taxes and subsidies" 2 ).

Diese Überlegung beruht auf der Tatsache, daß aufgrund der Mehrbelastung das monetäre Äquivalent einer Reduktion der Besteuerung das monetäre Äquivalent einer Steigerung des Transfervolumens überwiegt. Daher besteht für die belastete Gruppe ein größerer Anreiz zur Produktion politischen Drucks als für die begünstigte Gruppe. Auf diesem Wege wird die Vergünstigungsgruppe jegliche Belastung durch Stevern abbaven und gleichzeitig die Subventionierung der Steuerzahlergruppe einschränken. Dieser Prozeß endet, wenn nur noch eine Gruppe bestevert bzw. subventioniert wird ${ }^{3}$.

Die Erweiterung der Analyse von zwei Interessengruppen auf den Wettbewerb zwischen mehreren politisch aktiven Gruppen ${ }^{4}$ führt unter den genannten Bedingungen zu prinzipiell gleichen Resultaten. In diesem Zusammenhang ist die Annahme eines CournotNash-Prozesses bei der Produktion politischen Drucks realistischer, da hier geringe konjekturale Reaktionen zu erwarten sind.

1) Zur Charakterisierung dieser Konstellation findet sich auch die Bezeichnung "Cross-hauling". Vgl. Becker (1983), S. 389 .

2) Vgl. Becker (1983), S. 389 .

3) "Cross-hauling could remain only if pressure by both groups were more productive in obtaining subsidies than in reducing taxes." Becker (1985), S. 335.

4) Vgl. Becker (1983), S. $388 \mathrm{ff}$. und Becker (1985), S. $341 \mathrm{f}$. 


\subsection{Veränderung der Gruppengräße}

Aus den Überlegungen zur Mehrbelastung lassen sich die Effekte einer Veränderung der Gruppengröße auf die Höhe des politischen Drucks ableiten. Da sich mit einer steigenden Mitgliederzahl der individuelle Steuerbetrag zur Erhebung eines gegebenen Aufkommens mindert, erfolgt eine Reduktion der entsprechenden Mehrbelastung. Aus diesem Grund resultiert gemäß

(v.60) $\quad t_{\lambda}=\frac{I_{t}^{s} p_{m n}^{t}}{F^{\prime}}-\frac{F F^{\prime \prime}}{n^{t} F^{\prime 2}}>0$ für $p_{m n}^{t} \leqq 0$

mit zunehmender Gruppengröße eine Verminderung des politischen Drucks der Steuerzahlergruppe. Begünstigte Gruppen werden daher "prefer its subsidy to be financed by small taxes on many persons, even when that does not reduce the political efficiency of the taxed group"1). D.h. unabhängig von den Organisationskosten auf seiten der Steuerzahler ergibt sich als Konsequenz der Senkung der Mehrbelastung ein verminderter Einfluß und damit eine Erhöhung des Vergünstigungsvolumens.

In Analogie wird für die Vergünstigungsempfänger bei steigender Gruppengröße

(V.61) $\quad s_{\lambda}=\frac{I_{s}^{s} p_{m n}^{s}}{G^{\prime}}+\frac{G G^{\prime \prime}}{n^{s} G^{\prime 2}}>0$ für $p_{m n}^{s} \geq 0$ ermittelt, woraus eine Zunahme politischer Aktivitäten folgt.

1) Becker (1983), S. 384 . 
Da mit einer Zunahme der Anzahl der Gruppenmitglieder der individuelle Vorteil sinkt, muß die begünstigte Gruppe eine relativ geringe Mitgliederzahl aufweisen, sofern keine Effizienzsteigerungen durch die Umverteilung induziert werden. Diese überlegungen führen zu der Schlußfolgerung, daß politisch erfolgreiche Gruppen "tend to be small relative to the size of the groups taxed to pay their subsidies"1).

\section{Interpretation und Kritik der Ergebnisse}

Die abgeleiteten Ergebnisse stehen im Einklang mit der Erkenntnis der Pluralismustheorie ${ }^{2}$, daß sich die Interessengruppen im Rahmen eines Systems von ökonomischen Gegenkräften unter bestimmten Bedingungen neutralisieren können. Wenngleich die Konstruktion der "politischen" Budgetrestriktion formal eine Verbindung zwischen dem marktlichen und dem politischen Sektor herstellt, werden damit die Konsequenzen der gegebenen politischen Institutionen demokratischer Staatswesen nur unzureichend erfaßt ${ }^{3}$ ).

Eine wesentliche Schlußfolgerung der bisherigen Überlegungen besteht darin, daß die Rent-Seeking-Aktivitäten der am Distributionskonflikt beteiligten Interessengruppen im Wege eines funktionierenden Interessenwettbewerbs eine Effizienzsteigerung staatichen Handelns bewirken können.

1) Becker (1983), S. 385.

2) Vgl. dazu Bentley (1908) bzw. Truman (1971). Zum Konzept der "countervailing power" sei auf Galbraith (1956) verwiesen.

3) Die Analyse beruht auf der Annahme, daß "the political process operates as a veil through which the underlying strenghts of interest groups and their demands are revealed". Coughlin/Mueller/Murrell (1986), S. 1. 
Effiziente staatliche Maßnahmen kommen lediglich dann zustande, wenn die betrachteten Gruppen "equally large and skillful at producing political influence"1) sind. Unter dieser Bedingung übersteigen die Nutzengewinne der Begünstigten die Nutzenverluste der Belasteten, so daß die erhöhte politische Einflußnahme von seiten der Verteilungsgewinner zur Effizienzsteigerung beiträgt. Kann die belastete Gruppe jedoch diesen intrinsischen Nachteil durch eine effiziente Produktionstechnik von politischem Einfluß kompensieren, wird sie als die einflußreichere Gruppe eine sie speziell belastende Maßnahme verhindern, obgleich diese unter Allokationsaspekten wünschenswert ist ${ }^{2}$.

Die Ergebnisse des Interessenwettbewerbs werden entscheidend von der Intensität der Opposition bei gruppenspezifischen RentSeeking-Vorhaben bestimmt. Ihr Ausmaß hängt von der Höhe und Dispersion der Verteilungsverluste sowie vom Informationsstand der benachteiligten Individuen ab. Um den politischen Widerstand anderer Gruppen zu mindern, werden geringfügige Vorteile angestrebt und die entsprechenden Belastungen möglichst breit auf andere Gruppen gestreut. Werden bestimmte Gruppen nur unwesentlich schlechter gestellt, ist bei gegebenen Kosten politischer Einflußnahme nicht mit starker Opposition zu rechnen. Aus diesem Grund wird beispielsweise eine gezielte Belastung bestimmter bereits organisierter Interessengruppen weitgehend vermieden, da hier von starken politischen Abwehrreaktionen auszugehen ist. Eine weite Streuung der Nachteile erschwert die Formierung von Gruppen mit gegensätzlichen Interessen, da die individuelle Belastung vergleichsweise unbedeutend ist und die Beteiligung an politischen Gegenmaßnahmen nicht lohnt ${ }^{3}$.

1) Becker (1985), S. 344 .

2) Vgl. Becker (1985), S. 344 f. aber auch Schellhaass (1988), S. 105 bzw. Crew/Rowley (1988), S. 57.

3) Vgl. Folkers (1983a), S. 197. 
Gleichzeitig werden im Rahmen einer gezielten Informationspolitik die Gruppeninteressen in der öffentlichkeit meist mit der Wahrung übergeordneter gesellschaftlicher Interessen verbunden. Auf diese Weise soll den gruppenspezifischen Zielen auch bei Nichtmitgliedern Legitimität verliehen werden. So werden Interessenvertreter häufig zu Anwälten des Gemeinwohls, die mit Hinweis auf Begriffe wie "Sozialstaat", "Solidargemeinschaft", "soziale Verantwortung" und "soziale Gerechtigkeit" ihre distributiven Vorhaben zu rechtfertigen suchen ${ }^{1)}$.

Durch diese Informationsbeeinflussung wird eine "Illusion"2) über die begünstigenden Maßnahmen erzeugt, die manche RentSeeking-Projekte erst ermöglichen. Anstelle der für einen wirksamen Interessenwettbewerb erforderlichen Opposition gegen einzelne Gruppenprivilegien werden Anreize zur Forderung nach eigenen Sondervergünstigungen induziert, die auf einen Ausgleich für die Gewährung von Verteilungsgewinnen zugunsten der anderen Gruppe abzielen. In diesem Prozeß werden Rent-Seeking-Vorhaben mit relativ geringfügigen Vorteilen für spezifische Gruppen im Zeitablauf wiederholt, um unmittelbar nicht erreichbare Sonderprivilegien inkrementell durchzusetzen und daverhaft zu sichern ${ }^{3}$ ).

1) Vgl. v. Weizsäcker (1984), S. 138 und Windisch (1984), S. 588. Eine Gruppenforderung wird in der öffentlichen Diskussion eher akzeptiert, wenn sie sich auf die Aufrechterhaltung der bisherigen Position richtet. Der Wunsch nach neven Privilegien wird dagegen in aller Regel wenig Unterstützung außerhalb der betrachteten Interessengruppen finden.

2) Vgl. dazu insbesondere Buchanan (1967), S. $126 \mathrm{ff}$. bzw. auch Wagner (1976), S. $45 \mathrm{ff}$. bzw. Goetz (1977), S. $176 \mathrm{ff}$.

3) Vgl. Folkers (1983a), S. $197 \mathrm{f}$. Ineffiziente Maßnahmen sind zu erwarten, "if there is a highly unequal access to political influence". Becker (1985), S. 344. 
Unabhängig davon ergeben sich Allokationsverluste in Form der beim kollusiven Rent-Seeking verschwendeten Ressourcen, d.h. die Gruppen verfolgen ihre spezifischen Interessen "regardless of the consequences for total societal output"1). Dieser Zusammenhang wird in Abbildung 12 veranschaulicht, wobei das aus dem politischen Wettbewerb resultierende Gleichgewicht mit $G_{0}$ bezeichnet ist.

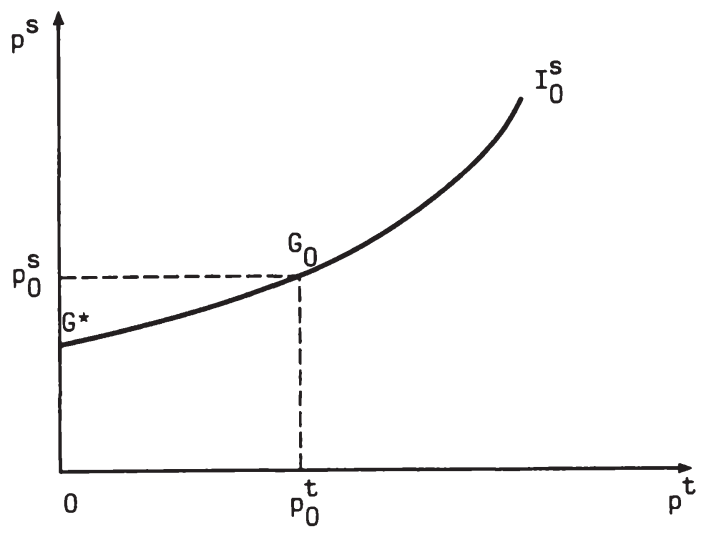

Abb. 12: Die allokativen Konsequenzen des Wettbewerbs um politischen Einfluß

Der gruppenspezifische Ressourceneinsatz für politische Einflußnahme ist ineffizient, da sich die Gruppen durch eine beiderseitige Verminderung ihrer Produktion politischen Drucks besserstellen könnten ${ }^{2}$ ).

1) Heijdra/Lowenberg/Mallick (1988), S. 306. Die Aktivitäten der Gruppen sind überwiegend "oriented to struggles over the distribution of income and wealth than to the production of additional output ..." . Olson (1982), S. 44.

2) In diesem Kontext impliziert Rent-Seeking eine Art X-Ineffizienz des politischen Entscheidungsverfahrens bei der Aushandlung des Verteilungsergebnisses. Vgl. Laux (1984), S. 21 . 
Das gleiche Verteilungsresultat wird bei $G^{*}$ realisiert, wobei in dieser Konstellation die Steverzahlergruppe keinen politischen Druck produzieren muß. Es werden mehr Ressourcen als notwendig für den Aushandlungsprozeß eines bestimmten Verteilungsergebnisses in Anspruch genommen. Da der gruppenspezifische Einfluß mit geringerem Ressourceneinsatz für die Produktion politischen Drucks aufrechtzuerhalten ist, können die Gruppenmitglieder ihre Einkommen durch die produktive Verwendung der im Verteilungskampf "gesparten" Ressourcen steigern ${ }^{1}$ ). Daraus folgt, daß eine Kooperation zwischen den Gruppen im Hinblick auf einen beiderseitigen Verzicht auf Rent-Seeking eine notwendige Voraussetzung darstellt, um "the wasteful expenditures on political pressure that result from the competition for influencen ${ }^{2}$ ) $z u$ verhindern. Es stellt sich allerdings die Frage, inwieweit bei Distributionskonflikten ein kooperatives Verhalten der Interessengruppen erwartet werden $\operatorname{kann}^{3}$ ).

Selbst wenn die Beeinflussungsversuche erfolgreich waren, läßt sich nicht die Schlußfolgerung ziehen, daß die auf den ersten Blick begünstigte Gruppe auch tatsächlich den Distributionsvorteil realisieren kann. Damit ist die Problematik angesprochen, in welchem Ausmaß die mit bestimmten Regelungen verbundenen Verteilungsvorteile den formal Begünstigten verbleiben bzw. Welche Verteilungseffekte aus den marktmäßigen Rückwirkungen einer Gewährung partikularer Sonderregelungen resultieren $^{4)}$.

1) "Therefore, efficiency would be raised if all groups could agree to reduce their expenditures on political influence." Becker (1985), S. 335.

2) Becker (1983), S. 388.

3) Vgl. dazu Kap. VII dieser Arbeit.

4) Vgl. Buchanan/Pauly (1970), S. $157 \mathrm{ff}$. 


\section{Die Konsequenzen marktmäBiger Verteilungseffekte}

Bei der Analyse der marktmäßigen Rückwirkungen infolge diskriminierender staatlicher Maßnahmen wird davon ausgegangen, daß die Interessengruppe s eine spezifische Steververgünstigung durchgesetzt hat. Die ökonomische Aktivität $x$ erfährt gezielt eine steuerliche Ausnahmebehandlung, d.h. es wird eine "spezielle Abweichung von der allgemeinen Steuernorm"1) betrachtet.

In Abbildung 13 ergibt sich ausgehend vom Gleichgewicht $A$ in Abhängigkeit vom individuellen Marginalsteuersatz des Gruppenmitgliedes ${ }^{2)}$ eine Drehung der Nachfragekurve von $D_{0}^{s}$ auf $D_{1}^{s}$. Wird ein konstanter Angebotspreis $p_{0}$ unterstellt, führt die mit der Vergünstigung verbundene Senkung des Nettopreises auf $p_{1} z u$ einer Ausdehnung der nachgefragten Menge von $x_{0}$ auf $x_{1}$.

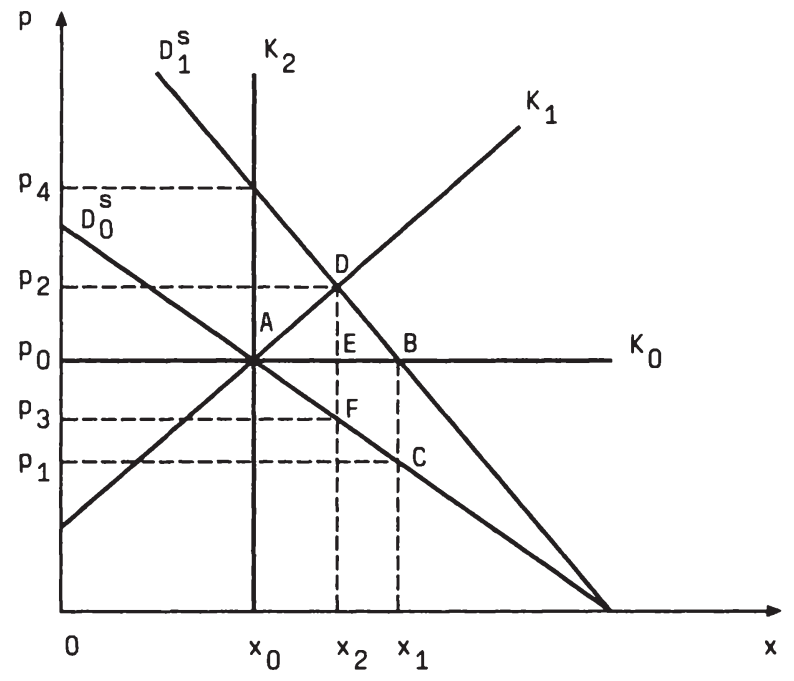

Abb. 13: Die Übertragung von Distributionsvorteilen
1) Folkers (1988), S. 216.
2) In Anlehnung an Folkers (1988), S. 223. 
Damit ergibt sich ein Verteilungsvorteil in Höhe von $\mathrm{P}_{0} B \mathrm{C}_{1}$, der allerdings um die mit marktlichen Substitutionseffekten verbundene Mehrbelastung $(A B C)$ vermindert wird. In aller Regel werden die zunehmenden Aktivitäten im privilegierten Bereich zu erhöhten Marktpreisen führen. Bei der Angebotsfunktion $K_{1}$ ist mit dem Preisanstieg auf $\mathrm{p}_{2}$ lediglich eine Ausdehnung der Nachfrage von $x_{0}$ auf $x_{2}$ verbunden. Der Verteilungsvorteil fällt damit teilweise $\left(p_{2} D E p_{0}\right)$ den Anbietern zu, so daß der begünstigten Gruppe infolge des Markteffektes ein Anteil von $\mathrm{P}_{0} \mathrm{EFP}_{3}$ verbleibt. Wenngleich im Zuge des marktmäßigen Anpassungsprozesses ebenso eine "Weitergabe" der Mehrbelastung erfolgt, müssen die Begünstigten dennoch deutliche Verluste gegenüber dem ursprünglichen Verteilungsvorteil hinnehmen ${ }^{1}$.

Im Extremfall eines vollständig unelastischen Angebotes $\mathrm{K}_{2}$ wird mit der Preiserhöhung auf $\mathrm{p}_{4}$ der Distributionsvorteil in vollem Umfang auf die Marktgegenseite übertragen ${ }^{2}$ ). Die Verteilungseffekte der Realisierung von Vergünstigungen werden somit entscheidend von den relevanten Angebots - und Nachfrageelastizitäten auf den jeweiligen Märkten bestimmt. Eine Verminderung der Angebotselastizität wird eintreten, wenn die Vergünstigung längerfristig gewährt und die Möglichkeit zur Realisierung potentieller Verteilungsgewinne durch die Marktgegenseite wahrgenommen wird ${ }^{3}$ ).

1) Folkers (1988), S. 222.

2) Die weitgehende Übertragung kann am Beispiel der Wohnungsbauförderung demonstriert werden. Bei einer unelastischen Angebotskurve wird sich die Zielsetzung einer Begünstigung für Wohnungserwerber in Preiserhöhungen niederschlagen. D.h. die Vorteile werden auf Baunternehmen bzw. Vorbesitzer von Grundstücken übertragen, die infolge der Erhöhung der Bautätigkeit bzw. der Wertsteigerung der Immobilien Mitnahmegewinne realisieren können. Vgl. White/White (1977), S. $111 \mathrm{ff}$. bzw. Mann (1987), S. $176 \mathrm{f}$. Im Zusammenhang mit staatlichen Maßnahmen zur Förderung unternehmerischer Innovationstätigkeit sei auf Herb (1988), S. $163 \mathrm{f}$. verwiesen.

3) Vgl. Folkers (1988), S. $224 \mathrm{f}$. 
Als Konsequenz der Analyse ergibt sich, daß diskriminierende staatliche Interventionen durch marktliche Mechanismen erodiert werden können ${ }^{1)}$. Wenngleich der vollständige Abbau des Verteilungsvorteils die Wiederherstellung horizontaler Gleichheit impliziert ${ }^{2}$, würde eine Abschaffung der begünstigenden Institution starke Opposition der betroffenen Gruppe induzieren, da reale Verluste hervorgerufen werden ${ }^{3)}$. Aus diesem Grund müssen dauernd neve und höhere Vorteile angestrebt werden, um die ursprüngliche Begünstigung aufrechtzuerhalten.

Die dynamischen Eigenschaften dieses Mechanismus fördern einen permanenten Verteilungskonflikt und wirken einer Reduzierung von Rent-Seeking-Vorhaben selbst oder gerade bei erfolgreich durchgesetzten staatlichen Regelungen entgegen. Die aus diesem Prozeß resultierenden Institutionen ziehen weitere Verteilungsaktivitäten organisierter Gruppen nach sich, die mit dem Hinweis auf die Wahrung "rechtmäßiger Besitzstände" gerechtfertigt werden. Die Effekte der Übertragung von Verteilungsvorteilen im Marktprozeß stellen eine Begründung für die Kumulation bzw. Akzeleration distributiv motivierter Institutionen dar. Der marktliche Übertragungsprozeß erklärt zugleich die Starrheit der Fiskalstruktur in bezug auf die Abschaffung von Verteilungsprivilegien, selbst wenn die Begünstigungen materiell nicht mehr existieren ${ }^{4}$ ).

1) Vgl. Tullock (1975), S. 677.

2) Vgl. dazu Feldstein (1976), S. 123.

3) "However, there is a large number of people who would suffer large transitional costs if the institution were terminated." Vgl. Tullock (1975), S. 677.

4) Folkers (1985), S. 285. 


\section{Zusammenfassung}

Interessengegensätze bei einzelnen staatlichen Maßnahmen sind die Ursache von Verteilungskonflikten. Vor diesem Hintergrund kann das Zustandekommen fiskalischer Institutionen aus dem Wettbewerb von Interessengruppen um politischen Einfluß erklärt werden. Die Analyse der widerstreitenden Verteilungsinteressen erfolgt auf der Grundlage eines Gleichgewichtsmodells, wobei die konkurrierenden Rent-Seeking-Bemühungen zweier Gruppen Art und Umfang der Verteilungsvorteile bestimmen. In diesem Zusammenhang wird explizit auf die Interdependenz zwischen den marktmäßigen Anpassungsreaktionen an einzelne statliche Institutionen und den interessenbedingten Einflußnahmeaktivitäten auf die Ausgestaltung der fiskalischen Regelungen eingegangen.

Als wesentlicher Bestimmungsfaktor des politischen Gleichgewichts der Distributionsinteressen erweist sich die staatliche Budgetrestriktion, die eine Verbindung zwischen Verteilungsgewinnen und -verlusten herstellt. Dabei wird unterstellt, daß Vergünstigungen zugunsten einer Gruppe mit entsprechenden Steuerbelastungen der anderen Gruppen einhergehen. Die Budgetrestriktion wirkt sich ebenfalls auf die Höhe des gruppenspezifischen Ressourceneinsatzes für Rent-Seeking aus. Aufgrund der Tatsache, daß eine Steigerung des politischen Einflusses einer Gruppe zu einer gleich hohen Verminderung des Einflusses der Opposition führt, ergibt sich in analoger Interpretation eine "politische" Budgetrestriktion.

Unter der Annahme, daß die politischen Entscheidungsträger lediglich als "Transfermakler" der organisierten Interessengruppen auftreten und die aus dem Wettbewerb um politischen Einfluß resultierenden Maßnahmen ausführen, wird das Gleichgewicht von Stevern und Vergünstigungen in erster Linie durch 
die gruppeninterne Effizienz bei der Produktion politischen Drucks, durch den politischen Einfluß der Opposition und durch die mit staatlichen Maßnahmen verbundene Mehrbelastung determiniert.

Als zentrales Ergebnis der Analyse des politischen Gleichgewichts ist festzuhalten, daß Gruppen, denen eine Steigerung der Produktionseffizienz politischen Drucks gelingt, ihre Vergünstigungen erhöhen bzw. ihre Steverlast vermindern können. Ebenso weisen erfolgreiche Gruppen in aller Regel eine geringere Mitgliederzahl auf als die Gruppierungen, welche diese Distributionsvorteile durch Steuern finanzieren müssen. Eine Verminderung des Vergünstigungsvolumens ergibt sich, wenn das Ausmaß der gruppenspezifischen Mehrbelastung zunimmt.

Die Schlußfolgerung, daß die unter dem Aspekt der Vorteilssuche stattfindenden politischen Einflußnahmeaktivitäten der Interessengruppen gleichfalls das Zustandekommen effizienter staatlicher Institutionen bewirken, ist unter Berücksichtigung bestimmter Unvollkommenheiten des Interessenwettbewerbs zu relativieren. Im Distributionskonflikt werden Ressourcen verschwendet, weil das gleiche Verteilungsergebnis mit einem geringeren Ressourceneinsatz für politischen Einfluß erzielbar ist. Die Vermeidung des "unproduktiven" Rent-Seeking der konkurrierenden Interessengruppen wird durch die Wirkungen der Übertragung von Verteilungsvorteilen im Wege marktmäßiger Anpassungen auf andere Gruppen erschwert, da Anreize zu neven Rent-Seeking-Vorhaben der formal begünstigten Gruppe induziert werden. Zur Erweiterung der Analyse müssen allerdings die gegebenen politischen Institutionen der Demokratie integriert und im Hinblick auf ihre Konsequenzen für den Wettbewerb der Verteilungsinteressen untersucht werden. 


\section{GRUPPENINTERESSEN UND POLITISCHES ENTSCHEIDUNGSVERHALTEN IN DER DEMOKRATIE}

Staatliche Institutionen werden nicht von einzelnen gesellschaftlichen Gruppen ausgehandelt, sondern im politischen Prozeß beschlossen. Im Rahmen eines positiven Erklärungsansatzes staatlicher Tätigkeit muß der Frage nachgegangen werden, inwieweit die gegebenen politischen Institutionen der Demokratie die Realisierung von gruppenspezifischen Verteilungsvorteilen bzw. einen wirksamen Interessenwettbewerb zulassen. Da jede staatliche Aktivität distributive Effekte hervorruft, wird das Verhalten aller am Zustandekommen staatlicher Regelungen beteiligten Akteure beeinfluBt ${ }^{1}$.

Nach der Darstellung der Funktionsweise des politischen Entscheidungssystems wird die Entwicklung der Statstätigkeit in der Bundesrepublik Deutschland aufgezeigt. Ausgehend von der Analyse staatlicher Umverteilungsaktivitäten im demokratischen Wahlprozeß werden Strategien zur Durchsetzung von Partikularinteressen untersucht. Dabei müssen auch die Zielsetzungen und Restriktionen von Politikern und Bürokraten als Anbietern von statlichen Institutionen berücksichtigt werden, die für den politischen Erfolg einzelner Rent-SeekingVorhaben entscheidende Bedeutung besitzen. Im Anschluß daran wird der Zusammenhang zwischen distributiven Gruppeninteressen und der Zunahme der Staatstätigkeit ${ }^{2}$ analysiert, wobei insbesondere die Effekte einer kollektiven Bereitstellung gruppenspezifisch begünstigender Güter betrachtet werden. Den Abschluß bildet eine Zusammenfassung der zentralen Ergebnisse dieses Kapitels.

1) "The essence of politics is redistribution and political conflicts center on matters of distribution." Brunner $(1978)$, S. 662 .

2) Vgl. Z.B. Lindbeck (1985), S. $309 \mathrm{ff}$. 


\section{Die Funktionsweise des politischen Entscheidungssystems}

Institutionelle Entscheidungen werden im Rahmen kollektiver Entscheidungsprozesse getroffen. Bei der Analyse des RentSeeking müssen vor allem die Interessen der am politischen Prozeß beteiligten Akteure, d.h. der Wähler, Interessengruppen, Politiker bzw. Bürokraten spezifiziert und die Konsequenzen der gegebenen politischen Restriktionen für die Ausgestaltung der staatlichen Maßnahmen untersucht werden. Dabei wird von der grundlegenden Annahme ausgegangen, daß die Individuen im politischen Bereich in gleicher Weise wie bei marktlichen Entscheidungen ihre eigenen Interessen verfolgen ${ }^{1}$.

\subsection{Die Nachfrage nach staatlichen Institutionen}

In demokratischen Systemen bringen die Wirtschaftssubjekte ihre Präferenzen in bezug auf staatliche Aktivitäten in erster Linie durch den Wahlprozeß zum Ausdruck. Dabei bestimmt die Zielsetzung der Nutzenmaximierung das individuelle Verhalten der Wähler, die als "Nachfrager" nach staatlichen Maßnahmen interpretiert werden. Bestimmte Gruppen von Wählern sind bei der Realisierung ihrer distributiven Vorstellungen nicht nur auf den Wahlprozeß angewiesen, sondern werden durch Organisierung in Interessengruppen zusätzlichen Einfluß erlangen. Dabei besitzt die Unterstützung von Interessengruppen den Vorteil, daß eine gezieltere Artikulation der gruppenspezifischen Interessen ermöglicht wird. Allerdings bedürfen die Zielsetzungen der "Verteilungskoalitionen"2), die auf die Durchführung oder Unterlassung bestimmter staatlicher Aktivitäten

1) Die Einführung gleicher Verhaltensannahmen für marktliches und politisches Handeln stellt die logische Konsequenz der Anwendung der ökonomischen Analyse auf politische Prozesse dar. Vgl. Buchanan (1987a), S. 587.

2) Olson (1982), S. 44 . 
im Interesse ihrer Mitglieder gerichtet sind, in demokratischen Staatswesen der Legitimation durch eine Wählermehrheit.

\subsection{Das Angebot an staatlichen Institutionen}

Das politische System der repräsentativen Demokratie wird durch die Konkurrenz von Parteien um die Stimmen der Wähler charakterisiert ${ }^{1)}$. Politiker können dabei als eigennützig handelnde Individuen interpretiert werden, die bestimmte staatliche Regelungen am "politischen Markt"2) anbieten, um Wählerstimmen zu gewinnen. Zur Regierungsbildung muß eine Partei über die Parlamentsmehrheit verfügen bzw. entsprechende Koalitionen eingehen. Das Interesse der Politiker an der Erhaltung bzw. Erlangung politischer Macht und die damit verbundene Möglichkeit zur Realisierung individueller Einkommens- und Prestigeziele bestimmt ihr Verhalten als Anbieter staatlicher Maßnahmen, die einzelnen Gruppen Zugang zu politischen Renten ermöglichen. Die Ausführung der im politischen Prozeß determinierten Entscheidungen wird der Bürokratie ${ }^{3)}$ übertragen, die ihrerseits einen gewissen Handlungsspielraum zur Verfolgung eigener Zielsetzungen (Macht, Einkommen, Sicherheit) besitzt. Beim Rent-Seeking privater Interessengruppen können Politiker und Bürokraten als "Transfermakler"4) im Rahmen ihrer legalen Kompetenzen begriffen werden, die unter Wiederwahlgesichtspunkten und zur Sicherung anderer Vorteile gruppenspezifische Distributionsvorhaben organisieren. Zur Systematisierung der Wirkungszusammenhänge bei der Entstehung staatlicher Regelungen kann Übersicht 2 dienen, in der die

1) Vgl. Downs (1968), S. $50 \mathrm{ff}$.

2) $\mathrm{Vgl}$. Anderson (1980), S. $132 \mathrm{ff}$.

3) Vgl. Niskanen (1968), S. 293 ff. bzw. die zusammenfassende Darstellung von Roppel (1979).

4) Vgl. McCormick/Tollison (1981), Kap. 2. 
"treibenden Kräfte der politischen Willensbildung"1) enthalten sind ${ }^{2}$ ).

Übersicht 2: Die Funktionsweise des politischen Entscheidungssystems

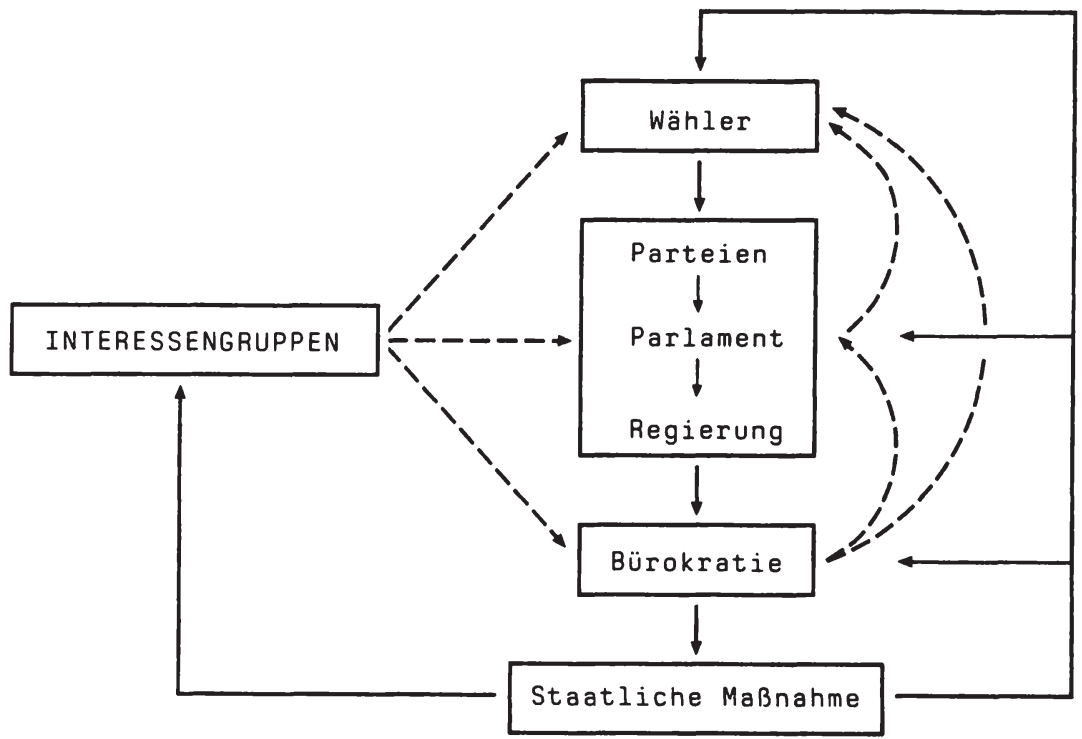

Die in Wahlen zum Ausdruck kommenden Wählerpräferenzen wirken auf die Entscheidungen des Parlaments und der Regierung. Die von der Bürokratie ausgeführten staatlichen Maßnahmen induzieren Rückkoppelungseffekte auf das Wahlverhalten und die distributiven Vorhaben der Interessengruppen. Neben privaten Verhaltensreaktionen sind allerdings auch Rückwirkungen auf das staatliche Entscheidungsverhalten zu erwarten.

1) Schmidt (1966), S. 215.

2) Dabei sind die unterbrochenen Linien als Einflußnahmemöglichkeiten zu interpretieren, während die aus staatlichen Maßnahmen resultierenden Rückwirkungen in Form durchgezogener Linien symbolisiert werden. Vgl. Atkinson/Stiglitz (1980), S. 296. 
Zur Durchsetzung ihrer jeweiligen Zielsetzungen werden die Interessengruppen sowohl Wähler als auch Politiker und Bürokraten beeinflussen, indem sie Verbandsarbeit leisten, eine parlamentarische Lobby unterhalten, Presse-bzw. Öffentlichkeitsarbeit betreiben und damit Ressourcen für vielfältige politische Aktivitäten aufwenden ${ }^{1}$ ). Trotz personeller Überschneidungen ${ }^{2}$ ) mit Wählern (und Politikern/Bürokraten) erscheint eine isolierte Analyse von Interessengruppen gerechtfertigt, da von ihnen eigenständige Anstäße ausgehen, die im Wahlprozeß nicht zur Geltung kommen. Insbesondere die mangelnde Information aller Beteiligten (z.B. bezüglich der Präferenzen der Wähler, der Lage einzelner Wirtschaftsbranchen bzw. der Konsequenzen vorgeschlagener oder verabschiedeter Regelungen im Bereich der Steverpolitik) führt dazu, daß die Möglichkeiten zur Einflußnahme durch Interessengruppen ständig an Bedeutung gewinnen. In dem Maße, in dem spezielle Interessengruppen die Informationsflüsse zwischen den Akteuren des politischen Entscheidungsprozesses im Hinblick auf die Gewährung vorteilhafter staatlicher Maßnahmen stevern können, sind systematische Abweichungen staatlicher Aktivitäten von den tatsächlichen Wählerpräferenzen zu vermuten. Aufgrund bestimmter Interessenverflechtungen werden Politiker und Bürokraten ihrerseits durch gezielte Informationsbeeinflussungen ihre Zielsetzungen mit Unterstützung einzelner Interessengruppen zu realisieren suchen ${ }^{3}$ ).

1) Die direkte Beeinflussung von Parlament bzw. Bürokraten hat den Vorteil, daß die gruppenspezifischen Anliegen nur einer im Vergleich zur Wählerschaft geringen Anzahl von Personen verdeutlicht werden müssen und damit die Einflußnahmekosten niedriger ausfallen.

2) Beispielsweise sind Politiker in aller Regel selbst Mitglieder von Interessengruppen. Ebenso treten Staatsbedienstete (Bürokraten) bei allgemeinem Wahlrecht auch als Wähler auf.

3) Die Konzeption des Rent-Seeking kann daher als Grundlage für die Entwicklung einer "interest-group theory of government and legislation" verstanden werden. Tollison (1982), S. 588 . 


\section{Distributionsinteressen und Entwicklung der Staatstätigkeit}

Nahezu jede staatliche Aktivität impliziert Distributionswirkungen. Neben expliziten Transferprogrammen mit offensichtlichem Umverteilungcharakter ( $z . B$. Transfers und Subventionen) bewirken auch andere staatliche Maßnahmen (z.B. Fördermaßnahmen. Sachtransfers, Regulierungen etc.) in aller Regel eine Benachteiligung bzw. Bevorzugung einzelner Individuen bzw. gesellschaftlicher Gruppen. Der Anreiz zur Entfaltung von Rent-Seeking-Aktivitäten "is directly related to the scope and range of governmental activity in the economy, to the relative size of the public sector" ${ }^{1)}$. Um einen Eindruck über die Ausdehnung der Staatstätigkeit in der Bundesrepublik Deutschland zu vermitteln, wird in Tabelle 3 die Entwicklung der staatlichen Ausgaben und Einnahmen dargestellt.

Die relative Bedeutung des öffentlichen Sektors in marktwirtschaftlich organisierten Wirtschaftssystemen kann sowohl über staatliche Ausgabengrößen als auch über Einnahmengrößen quantifiziert werden. In der politischen Diskussion dienen meist die Staatsquote und die Abgabenquote als Indikatoren für den Grad der Statstätigkeit. Die Staatsquote wird als Verhältnis der gesamten staatlichen Ausgaben zum Bruttosozialprodukt zu (jeweiligen) Marktpreisen definiert. Die Staatsausgaben umfassen sowohl die staatlichen Realausgaben als auch die Transferzahlungen. Da die staatlichen Übertragungszahlungen weitere Ausgaben im privaten Wirtschaftssektor bewirken, kann die Staatsquote nicht als Maß für die Inanspruchnahme des Sozialproduktes durch den öffentlichen Sektor verwendet werden ${ }^{2}$ ).

1) Buchanan (1980a), S. 4.

2) Zu methodischen Problemen bei der Bildung staatswirtschaftlicher Quoten und Beziehungszahlen sei auf Folkers (1979) sowie Gantner (1984) verwiesen. 
Tab. 3: Ausgaben und Einnahmen des Staates ${ }^{1)}$ (Mrd. DM)

\begin{tabular}{|c|c|c|c|c|c|}
\hline \multirow[t]{2}{*}{$\operatorname{Jahr}^{2}{ }^{2}$} & \multirow{2}{*}{$\begin{array}{l}\text { Bruttosozial- } \\
\text { produkt in } \\
\text { jeweiligen } \\
\text { Preisen }\end{array}$} & \multicolumn{2}{|c|}{ Ausgaben } & \multicolumn{2}{|c|}{ Einnahmen } \\
\hline & & absolut & $\%$ von $(2)$ & absolut & $\%$ von $(2)$ \\
\hline (1) & (2) & (3) & (4) & (5) & (6) \\
\hline $\begin{array}{l}1960 \\
1961 \\
1962 \\
1963 \\
1964 \\
1965 \\
1966 \\
1967 \\
1968 \\
1969 \\
1970 \\
1971 \\
1972 \\
1973 \\
1974 \\
1975 \\
1976 \\
1977 \\
1978 \\
1979 \\
1980 \\
1981 \\
1982 \\
1983 \\
1984 \\
1985 \\
1986 \\
1987 \\
1988\end{array}$ & $\begin{array}{r}303,00 \\
331,40 \\
360,50 \\
382,10 \\
419,60 \\
458,20 \\
487,40 \\
493,70 \\
533,70 \\
597,80 \\
675,70 \\
751,80 \\
825,10 \\
918,90 \\
985,60 \\
1029,40 \\
1126,20 \\
1199,20 \\
1291,60 \\
1396,60 \\
1485,20 \\
1545,10 \\
1597,10 \\
1680,40 \\
1769,90 \\
1844,30 \\
1945,20 \\
2020,10 \\
2128,90\end{array}$ & $\begin{array}{r}99,66 \\
113,35 \\
129,58 \\
140,38 \\
153,17 \\
170,23 \\
181,59 \\
193,56 \\
211,08 \\
233,33 \\
264,13 \\
304,30 \\
340,99 \\
386,45 \\
444,84 \\
509,09 \\
546,20 \\
582,68 \\
620,76 \\
669,75 \\
722,36 \\
766,17 \\
795,98 \\
816,44 \\
848,97 \\
875,45 \\
912,23 \\
948,20 \\
988,58\end{array}$ & $\begin{array}{l}32,9 \\
34,2 \\
35,9 \\
36,7 \\
36,5 \\
37,2 \\
37,3 \\
39,2 \\
39,6 \\
39,0 \\
39,1 \\
40,5 \\
41,3 \\
42,1 \\
45,1 \\
49,5 \\
48,5 \\
48,6 \\
48,1 \\
48,0 \\
48,6 \\
49,6 \\
49,8 \\
48,6 \\
48,0 \\
47,5 \\
46,9 \\
47,0 \\
46,4\end{array}$ & $\begin{array}{l}100,98 \\
114,39 \\
126,01 \\
134,43 \\
145,78 \\
156,36 \\
168,71 \\
173,80 \\
192,00 \\
223,59 \\
246,83 \\
282,16 \\
313,86 \\
370,47 \\
403,10 \\
421,52 \\
473,93 \\
517,60 \\
547,89 \\
588,86 \\
629,75 \\
655,27 \\
679,55 \\
705,23 \\
743,81 \\
780,02 \\
810,89 \\
842,56 \\
878,47\end{array}$ & $\begin{array}{l}33,3 \\
34,5 \\
35,0 \\
35,2 \\
34,7 \\
34,1 \\
34,6 \\
35,2 \\
36,0 \\
37,4 \\
36,5 \\
37,5 \\
38,0 \\
40,3 \\
40,9 \\
40,9 \\
42,1 \\
43,2 \\
42,4 \\
42,2 \\
42,4 \\
42,4 \\
42,5 \\
42,0 \\
42,0 \\
42,3 \\
41,7 \\
41,7 \\
41,3\end{array}$ \\
\hline
\end{tabular}

Quelle: Sachverständigenrat zur Begutachtung der gesamtwirtschaftlichen Entwicklung, Jahresgutachten 1988/89, Tabelle 33, S. 274 f.

1) Nach der Abgrenzung der Volkswirtschaftlichen Gesamtrechnung.

2) Ab 1986 vorläufige Ergebnisse. 
Gleichwohl ist sie ein Indiz dafür, inwieweit Kreislaufströme "über den öffentlichen Sektor laufen und damit in irgendeiner Weise vom Staat kontrolliert werden"1). Tabelle 3 verdeutlicht, daß die Staatsquote von 32,9\% im Jahre 1960 auf 46,4\% im Jahre 1988 angestiegen ist. In dieser Maßzahl kommt die Statstätigkeit allerdings nur insoweit zum Ausdruck, als sie mit Ausgaben verbunden ist ${ }^{2}$. Hinzu kommen die zahlreichen regulativen Eingriffe in Form von Vorschriften, Auflagen, Verboten etc., die in ähnlicher Weise den privaten Sektor beeinflussen, sich jedoch nicht auf der Ausgabenseite des Budgets niederschlagen ${ }^{3}$ ).

Wird auf der Einnahmenseite das Verhältnis der Summe aus Steuern und Sozialbeiträgen zum Bruttosozialprodukt gebildet, ergibt sich die Abgabenquote. Dieser Indikator bringt zum Ausdruck, in welchem Ausmaß dem privaten Sektor zunächst aufgrund gesetzlicher Verpflichtungen Ressourcen entzogen und auf den Staat übertragen werden ${ }^{4}$ ). In der Regel ist diese Quote ökonomisch aussagekräftiger als die Steverquote, da die Beiträge zur gesetzlichen Sozialversicherung ebenfalls den Charakter von Zwangsabgaben besitzen. Darüber hinaus bestehen

1) Folkers (1979), S. 459.

2) Versuche zur Bildung einer sogenannten "unsichtbaren" Staatsquote scheitern an der Problematik der Operationalisierung und Quantifizierung dieser Tätigkeiten.

3) Auf die zunehmende Bedeutung dieser Form der Staatstätigkeit weist auch der Sachverständigenrat zur Begutachtung der gesamtwirtschaftlichen Entwicklung im Jahresgutachten $1988 / 89$, S. 149 hin.

4) Da der Staat jedoch einen Teil seiner Einnahmen in Form von Transfers wieder an den privaten Sektor zurückgibt, "ist aus der Abgabenquote nicht zu erkennen, in welchem Umfang Einkommensteile endgültig aus der privaten in die öffentliche Verwendung übergegangen sind". Wissenschaftlicher Beirat beim Bundesministerium der Finanzen (1976), S. 860. 
vielfältige Substitutionsbeziehungen ${ }^{1}$ ) zwischen allgemeiner Steuerfinanzierung und spezieller Finanzierung über Sozialversicherungsbeiträge. Wie Tabelle 3 belegt, stieg die Abgabenquote von 33,3\% im Jahr 1960 bis zum Jahr 1988 auf $41,3 \%$ an. Allerdings muß auch hier berücksichtigt werden, daß zahlreiche steverliche Maßnahmen nicht im öffentlichen Haushalt erscheinen, obwohl sie einen erheblichen Staatseinfluß implizieren. Hierzu zählt insbesondere der Verzicht auf mögliche Steuereinnahmen ${ }^{2}$, der aus Steuerbefreiungen bzw. der Gewährung von steuerlichen "Schlupflöchern" zugunsten einzelner gesellschaftlicher Gruppen resultiert.

Diese Überlegungen verstärken den Eindruck, daß der Umfang der Staatstätigkeit bei Berücksichtigung der Substitutionsmöglichkeiten $z$ wischen budgetären und außerbudgetären staatlichen Maßnahmen möglicherweise noch stärker zugenommen hat, als es im Anstieg der Staats- bzw. Abgabenquote zum Ausdruck kommt. Aus der Rent-Seeking-Perspektive ist zu vermuten, daß gerade das Distributionsmotiv das Zustandekommen der zahlreichen statlichen Institutionen und damit die Ausdehnung des öffentlichen Sektors in den letzten drei Jahrzehnten begünstigt hat.

1) Beispielsweise können Einnahmenzuwächse bei der Sozialversicherung durch direkte Beitragserhöhungen oder durch erhöhte steverfinanzierte Staatszuschüsse realisiert werden.

2) Vgl. die Untersuchung von Surrey (1973) über die Entwicklung der "unsichtbaren" Steuervergünstigungen in den USA. 


\section{Gruppeninteressen in der Mehrheitsdemokratie}

In demokratischen Staatswesen wird das Zustandekommen politischer Entscheidungen in idealtypischer Betrachtung durch einen wirksamen Wahlmechanismus erklärt. Ausgehend von der Analyse demokratischer Mehrheitsentscheidungen bei Verteilungsfragen werden zunächst alternative Hypothesen über Richtung und Ausmaß der Einkommensumverteilung im politischen Prozeß diskutiert. Bestimmte Unvollkommenheiten des Wahlprozesses eröffnen Möglichkeiten zur Durchsetzung von Sonderinteressen. Zunächst werden die Wirkungen einer interessenbedingten Einflußnahme auf die Ergebnisse des kollektiven Entscheidungsprozesses spezifiziert. Auf dieser Grundlage wird die Bedeutung der Interdependenz von distributiven Gruppeninteressen und politischem Entscheidungsverhalten für die Wirksamkeit des Wettbewerbs zwischen Interessengruppen aufgezeigt. Anschließend wird auf das Verhalten der Bürokratie beim Rent-Seeking privater Interessengruppen eingegangen.

\subsection{Mehrheitswahlsystem und staatliche Umverteilung}

Theoretische Erklärungsansätze staatlicher Umverteilung in der Demokratie gelangen zu einer Reihe widersprüchlicher Hypothesen über Ausmaß und Richtung der Einkommensumverteilung durch den Wahlmechanismus ${ }^{1)}$. Nach der Analyse von Downs ${ }^{2}$ kann die Umverteilung in demokratischen Systemen als Resultat einer ungleichen Einkommensverteilung bei Gleichverteilung der Wählerstimmen interpretiert werden. Unter der Annahme, daß gesellschaftliche Entscheidungen auf der Grundlage der einfachen Mehrheitsregel getroffen werden, bestimmt bei eindimensionalem

1) Vgl. die umfassende Untersuchung von Knappe (1980).

2) Vgl. Downs (1968), S. $194 \mathrm{ff}$. 
Alternativenraum und eingipfligen Präferenzordnungen der Medianwähler ${ }^{1)}$ das kollektive Ergebnis. Liegt das Einkommen des Medianwählers unter dem gesellschaftlichen Durchschnittseinkommen, besitzt die Wählermehrheit ein unterdurchschnittliches Einkommen. Bei linkssteiler Verteilung der Einkommen kann die Wählermehrheit die Bezieher überdurchschnittlicher Einkommen überstimmen und im Zuge des Wahlprozesses eine Umverteilung zu ihren Gunsten realisieren.

In diesem Zusammenhang ist auch eine Zunahme fiskalischer Umverteilungsaktivitäten zu erwarten ${ }^{2}$ ). Dabei wird ein SteuerTransfer-System unterstellt, bei dem das Aufkommen einer proportionalen Einkommensteuer auf den (alleinigen) Produktionsfaktor Arbeit in Form gleicher Transfers an alle Individuen zurückgegeben wird. In dieser Konstellation profitieren die einkommensschwachen Schichten von einer Koalition mit dem Medianwähler, der das Ausmaß der Umverteilung festlegt. Allerdings wird keine Nivellierung der Einkommensverteilung ${ }^{3}$ erfolgen, da die Mitglieder der Mehrheitskoalition die negativen

1) Vgl. Black (1948), S. 23 ff. Die Erfordernis der Eingipfligkeit der Präferenzordnung für ein Gleichgewicht ist dadurch gekennzeichnet, daß für eine bestimmte Reihung der Entscheidungsalternativen bei je drei beliebigen Alternativen die mittlere zumindest einer der beiden äußeren Wahlmöglichkeiten vorgezogen wird. Vgl. Arrow (1963) bzw. auch die zusammenfassende Darstellung bei Mueller (1979).

2) Vgl. dazu Meltzer/Richard (1978), S. $111 \mathrm{ff}$. und (1981), S. $914 \mathrm{ff}$. Sowie (1983), S. $403 \mathrm{ff}$. Zur Sicherung des Erfordernisses eingipfliger Präferenzen wird lediglich über die Höhe des Steversatzes, jedoch nicht über die Struktur des Stever-Transfer-Systems abgestimmt.

3) Beispielsweise kann auch eine progressive Besteverung als Instrument der Einkommensumverteilung beschlossen werden. Hier ergeben sich Grenzen der Einkommensnivellierung aus der Tatsache, daß die Individuen bei vorteilhafter Entwicklung des eigenen Einkommens nicht selbst durch eine extreme Progression belastet werden wollen. 
Anreizeffekte einer zunehmenden Steuerbelastung auf die Höhe der "Verteilungsmasse" berücksichtigen werden. Eine wachsende Diskrepanz zwischen Durchschnitts- und Medianeinkommen impliziert eine verstärkte Einkommensungleichheit, die eine fiskalische Umverteilung zugunsten der unteren Einkommensgruppen bewirkt.

Diese Umverteilungstendenz folgt auch aus einem ähnlichen Ansatz von Peltzman ${ }^{1)}$, der auf der Annahme einer linkssteilen Einkommensverteilung bei gegebener Besetzung der Einkommensklassen beruht ${ }^{2}$ ). Dabei treten Politiker als "Transfermakler" auf, die um Wählerstimmen werben und auf diesem Wege den höchsten Nettostimmenzuwachs erringen können. Darüber hinaus sind allerdings auch die Einkommensunterschiede innerhalb der begünstigten Gruppen von Bedeutung. Eine zunehmende Umverteilung ist danach zu erwarten, wenn die Politiker Gruppen mit geringen Einkommensunterschieden und gleichen Interessen vorfinden. Im Gegensatz zur Begründung staatlicher Umverteilung durch eine stärkere Ungleichheit der Einkommensverteilung ergibt sich hier die gegenteilige Schlußfolgerung: "greater equality of private incomes increases the demand for political redistribution" 3 ). Aufgrund der fortschreitenden Arbeitsteilung, der beruflichen Spezialisierung und der regionalen Mobilität der Individuen wird die Organisation von speziellen Interessen ermöglicht, die sich in Verbindung mit dem

1) Vgl. Peltzman (1980), S. $209 \mathrm{ff}$.

2) Wenngleich der Umverteilungsaspekt als treibende Kraft für die Zunahme der Staatstätigkeit betont wird, findet nicht jede Distributionsmaßnahme die Unterstützung der einkommensschwachen Schichten, da sie von den gesamtwirtschaftlichen Rückkoppelungseffekten einer reduzierten Konsumnachfrage der zu stark besteuerten Bezieher hoher Einkommen negative Auswirkungen befürchten.

3) Peltzman (1980), S. 209. 
allgemeinen Wahlrecht in steigenden Umverteilungsmaßnahmen niederschlagen. Diese Entwicklung wird durch die Heterogenisierung zwischen einzelnen Gruppen bei gleichzeitiger Homogenisierung der Präferenzen innerhalb der Gruppen unterstützt ${ }^{1}$ ).

Nach einer anderen, unter der Bezeichnung "Director's Law" diskutierten Hypothese wird hingegen vermutet, daß der demokratische Prozeß zu einer Umverteilung zugunsten der mittleren Einkommensklassen führe ${ }^{2)}$. Zur Begründung wird auf die unterschiedliche Wahlbeteiligung einzelner Wählerschichten verwiesen, die eine Abweichung der tatsächlichen von der nominellen Wählerstimmenverteilung verursacht. Außerdem können Wahlerfolge nur mit den Stimmen der Individuen im mittleren Einkommensbereich erzielt werden, so daß diese Gruppe mit den unteren bzw. oberen Einkommensbeziehern koalieren und damit von beiden Gruppen Sondervorteile erlangen kann. Andererseits existiert neben der Vorstellung, daß die Verteilungsrichtung im demokratischen Prozeß vom oberen Mittelstand zum unteren Mittelstand verlaufe ${ }^{3)}$, auch die Behauptung einer Umverteilung von "arm" zu "reich" aufgrund der unterschiedlichen "Markt- und Meinungsmacht" einzelner Wählergruppen ${ }^{4}$ ).

1) Zu empirischen Untersuchungen dieser Hypothese vgl. z.B. Fratianni/Spinelli (1982), S. $221 \mathrm{ff}$. bzw. Gabriel/Loderer (1988), S. $267 \mathrm{ff}$.

2) Vgl. dazu Stigler (1970), S. $1 \mathrm{ff}$.

3) Vgl. z.B. Külp (1975), S. $234 \mathrm{ff}$, der eine "Umverteilung zugunsten der nicht ganz Armen und zu Lasten der nicht ganz Reichen" konstatiert.

4) Vgl. Peppard Jr. (1976), S. 1 ff. In diesem Zusammenhang sei auch auf marxistische Thesen verwiesen, die eine Ausbeutung der "Arbeiter" durch die "Kapitalisten" vermuten. Vgl. dazu z.B. Fleischmann (1974), S. 142 ff. 
Als Konsequenz dieser widersprüchlichen Erklärungsansätze ist festzuhalten, daß eine ungleiche Einkommensverteilung das Zustandekommen staatlicher Umverteilungsmaßnahmen beeinflußt, während die Umverteilungsrichtung im politischen Prozeß unbestimmt ist. Die Möglichkeit zur Bildung von Koalitionen ist ein wesentlicher Bestandteil aller demokratischen Abstimmungsverfahren und damit unabhängig von der konkreten Einkommenssituation einzelner Individuen. Die inhärente Instabilität des demokratischen Wahlprozesses bei Verteilungsfragen beruht darauf, daß Wählerkoalitionen einmal getroffene Mehrheitsentscheidungen bei periodischen Abstimmungen wechselseitig außer Kraft setzen können. Bei rationalem Verhalten wird die Mehrheit die Minderheit ausbeuten, indem sie Transfers von der Minderheit beschließt und sie unter ihren Mitgliedern aufteilt. Auf diese Weise existiert bei Verteilungsentscheidungen für jedes politische Ergebnis ein anderes, das bei Gültigkeit der Mehrheitsregel präferiert wird ${ }^{1)}$. Aufgrund der zyklischen Mehrheitskoalitionen ergibt sich kein stabiles Gleichgewicht ${ }^{2}$.

Ein Blick auf die Realität politischer Umverteilung in demokratischen Systemen bestätigt dieses Resultat offensichtlich nicht. Wenngleich die Ungleichheit der Einkommensverteilung einen nicht unbedeutenden Bestimmungsfaktor für die Zunahme fiskalischer Umverteilungsmaßnahmen darstellt, ist damit die Existenz vieler staatlicher Institutionen mit distributivem Charakter nicht erklärbar.

1) Vgl. Brennan/Buchanan (1981), S. 118 und Blankart (1983), S. 156 bzw. Laux (1984), S. $93 \mathrm{ff}$.

2) Wird darüber hinaus über mehrdimensionale Entscheidungen (z.B. Steuer- und Ausgabenstruktur) abgestimmt, ist die Existenz einer Gleichgewichtslösung aufgrund zyklischer Präferenzen der Individuen ohnehin nicht zu erwarten. Zur Kritik am Medianwähler-Modell vgl. Rowley (1984), S. 104 ff. bzw. Inman (1987), S. 710. Vgl. allerdings auch Abrams/ Lewis (1987), S. $127 \mathrm{ff}$. und Holcombe (1989), S. $115 \mathrm{ff}$. 
Beispielsweise ist zu fragen, warum die staatliche Umverteilung nicht in einem einheitlichen und transparenten system stattfindet, sondern in Gestalt einer Vielzahl von gruppenspezifischen Einzelregelungen durchgeführt wird. Viele der staatlich determinierten Regelungen sind dabei auf einzelne in einflußreichen Interessengruppen organisierte Wählerschichten gerichtet, die sich weniger durch einkommensmäßige Homogenität als vielmehr durch Gemeinsamkeiten hinsichtlich Beruf, Wirtschaftsbranche bzw. anderer gruppenbezogener Merkmale (Landwirte, Beamte etc.) auszeichnen und die sich nicht auf eine politische Mehrheit stützen können ${ }^{1)}$. Auch ist die Struktur des Steversystems nicht durch eine einfach zu erklärende Progression gekennzeichnet, sondern weist eine fülle unsystematischer und widersprüchlicher Begünstigungen zugunsten einzelner ökonomischer Tatbestände bzw. spezieller Interessengruppen $a \cup f^{2)}$.

Aus der Rent-Seeking-Perspektive ist zu vermuten, daß die aktuelle Vielfalt staatlicher Institutionen das Ergebnis einer erfolgreichen politischen Einflußnahme spezieller Gruppen ist, denen auf diese Weise die Sicherung bzw. Realisierung von gruppenspezifischen Verteilungsprivilegien für ihre Mitglieder gelungen ist. Allerdings läßt die Berücksichtigung der divergierenden Gruppeninteressen bei vielen politischen Programmen mit der Folge von widersprüchlichen und ineffizienten Sonderregelungen eine eindeutige Identifikation der tatsächlichen Gewinner bzw. Verlierer der staatlichen Interventionen nicht zu.
1) Vgl. Tullock (1971b), S. $379 \mathrm{ff}$.
2) Vgl. Folkers (1986a), S. 22. 
3.2. Die Durchsetzung von Sonderinteressen im Vergünstigungsstaat

Das Zustandekommen von partikularen Verteilungsvorteilen muß in Verbindung mit den politischen Institutionen in demokratischen Staatswesen betrachtet werden. "One of the major activities of modern governments is the granting of special privileges to various groups of politically influential people"1).

Im System der repräsentativen Demokratie wird unterstellt, daß Politiker das Ziel der Stimmengewinnung verfolgen. Der Wettbewerb um Wählerstimmen erzwingt unter idealtypischen Bedingungen eine Anpassung an die Wählerpräferenzen. Ansatzpunkte für die Einflußnahme von Interessengruppen ergeben sich aus der Tatsache," daß Wähler und Politiker gewisse Informationsdefizite aufweisen. Im folgenden werden die Wirkungen einer gezielten Beeinflussung ${ }^{2}$ ) des Verhaltens von Wählern und Politikern untersucht. Anschließend wird die Möglichkeit zur gemeinsamen Durchsetzung von Sonderinteressen im politischen Prozeß dargestellt.

\subsubsection{Informationsbeeinflussung und Wählerverhalten}

Interessengruppen vermitteln Informationen an ihre Mitglieder bzw. Wähler. Die Bedeutung einzelner Gruppen findet ihre Begründung im unterschiedlichen Informationsstand von organisierten und nichtorganisierten Wählerschichten. Zur Beeinflussung werden Interessengruppen ihre Informationen gezielt einsetzen und

1) Tullock (1975), S. 671.

2) Neben der Informationspolitik werden Interessengruppen auch ihre Marktmacht einsetzen bzw. finanzielle Zuwendungen an Parteien gewähren. Vgl. dazu Bernholz (1973), S. 865 bzw. (1975), S. 101 sowie Crain/Shughart II/Tollison (1988), S. 243 . 
vor allem nur die Informationen bereitstellen, die der Durchsetzung ihrer Ziele nützen bzw. zumindest nicht im Wege stehen.

Die politische Uninformiertheit eines Wirtschaftssubjektes findet seine Begründung in der Existenz von Kosten der Informationsgewinnung. Zur Beurteilung der Konsequenzen staatlicher Maßnahmen für die eigene ökonomische Position müssen monetäre und nichtmonetäre Ressourcen (z.B. Zeit) aufgewendet werden. Wenn der marginale Ressourceneinsatz zur Informationsbeschaffung den erwarteten Ertrag überwiegt, unterliegen die Individuen einer "rationalen Informationsbeschränkung"1). Der einzelne Wähler hat einen geringen Anreiz sich zu informieren, da er mit seiner Stimme nur unwesentlichen Einfluß auf den Wahlausgang ausüben kann. Aus diesem Grund ist davon auszugehen, daß der Informationsstand des Wählers über die Auswirkungen bestimmter statlicher Maßnahmen als äußerst gering zu veranschlagen ist.

Die rationale Informationsbeschränkung und eine interessenbezogene Informationsbeeinflussung durch Interessengruppen ermöglichen einen Prozeß der "Informationsverschmutzung", der eine beträchtliche Ungleichheit politischer Einflußnahme hervorruft. Die Wirkungsweise einer solchen Informationsweitergabe soll anhand der Entscheidungssituation eines Wählers illustriert werden. Zur Ermittlung der Konsequenzen einer Entscheidung für eine staatliche Maßnahme A bzw. B müssen Informationen erworben werden. Der Wähler sieht sich zwei Arten von Informationen gegenüber. Während Informationen vom Typ A die Wahrscheinlichkeit einer Entscheidung zugunsten von Regelung A erhöhen, implizieren Informationen vom Typ B die analoge Wirkung für die Alternative $\mathrm{B}^{2}$ ).

1) Vgl. Downs (1968), S. 255 ff.

2) Vgl. insbesondere Bartlett (1973), S. 27 ff. 
In Abbildung 14 werden die Grenznutzen $D_{0}^{A}\left(D_{0}^{B}\right)$ bzw. die Grenzkosten $K_{0}^{A}\left(K_{0}^{B}\right)$ der Beschaffung von Informationen vom Typ $A$ (B) dargestellt. Aus Gründen der Vereinfachung wird unterstellt, daß für beide Informationstypen identische Nachfrage- bzw. Angebotsbeziehungen vorliegen. Die effiziente Menge an Informationen ergibt sich, wenn die Grenzkosten der Informationsgewinnung ihren Grenzerträgen entsprechen ${ }^{1)}$. Damit wird im Ausgangsgleichgewicht $G_{0}$ eine gleich hohe Menge $\left(A_{0}=B_{0}\right)$ an Informationen über beide Maßnahmen erworben.

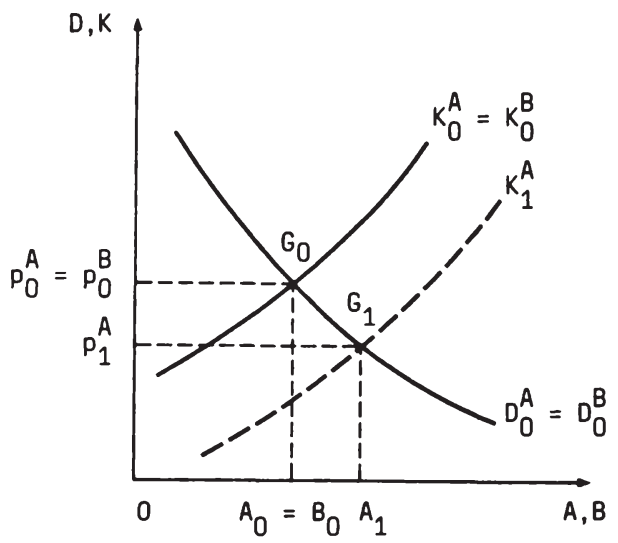

Abb. 14: Die Wirkungen einer interessenbedingten Informationsbeeinflussung

In der vorliegenden Konstellation ist eine spezielle Gruppe an der Verabschiedung der staatlichen Regelung $A$ interessiert, da auf diese Weise ein gruppenspezifischer Verteilungsvorteil resultiert.

1) Vgl. auch Laux (1984), S. $120 \mathrm{ff}$. 
Die Zielsetzung der Interessengruppe besteht nun darin, solche Informationen bereitzustellen, die eine politische Entscheidung im Interesse ihrer Mitglieder fördern. Da die betrachtete Gruppe die Institution $A$ wünscht, wird sie gezielt Informationen vom Typ A zu subventionierten Preisen zur Verfügung stellen ${ }^{1}$. Mit der Verminderung der Kosten des Erwerbs dieser Informationen ergibt sich bei unveränderten Präferenzen des Wählers eine Veränderung seines Informationsverhaltens. Die durch die Einflußnahme der Interessengruppe induzierte Kostensenkung $\left(K_{1}^{A}\right)$ für Informationen vom Typ A bewirkt einen Anstieg der Nachfrage nach diesen Informationen von $A_{0}$ auf $A_{1}$. Da der Erwerb von Informationen des Typs $B$ nicht beeinfluBt wird, ergibt sich hier keine Veränderung des. individuellen Informationsstandes. Als Resultat der interessenbezogenen Beeinflussung ist festzuhalten, daß sich im neven Gleichgewicht $G_{1}$ nicht nur die Gesamtmenge an Informationen $\left(A_{1}+B_{0}\right)$ sondern auch die Struktur der Informationen zugunsten einer Entscheidung für die Maßnahme $A$ verändert nat ${ }^{2}$ ).

Aufgrund der Tatsache, daß Interessengruppen in erster Linie partikulare Vorteile $e^{3)}$ zugunsten ihrer klientel anstreben, werden sie vorwiegend Informationen über die staatlichen Vorhaben beschaffen, die die Interessen ihrer speziellen Gruppe berühren bzw. bestimmte (positive oder negative) Konsequenzen hervorrufen. Aus diesem Grund werden organisierte Gruppen besser bezüglich der sie direkt betreffenden Maßnahmen informiert sein als andere Gesellschaftsmitglieder, allerdings hinsichtlich anderer staatlicher Aktivitäten weiterhin hohe Informationsdefizite aufweisen.

1) Vgl. Bartlett (1973), S. $31 \mathrm{f}$.

2) "When knowledge is imperfect, effective political action requires the use of economic resources to meet the cost of information." Downs (1957), S. 141.

3) Vgl. Blankart (1983), S. 157. 
Damit resultiert eine Asymmetrie rationaler Informationsbeschaffung ${ }^{1)}$. Während die Begünstigten spezieller staatlicher Maßnahmen aufgrund der individuellen Vorteile gut informiert sind, steht dieser Gruppe eine Mehrheit der an einzelnen Regelungen relativ unbeteiligter und daher unzureichend informierter Konsumenten/Steuerzahler gegenüber. Die Subventionierung spezifischer Informationen induziert ein verändertes Wahlverhalten, das den Politiker zur Realisierung der begünstigenden Maßnahmen veranlaßt.

Die Uninformiertheit auf seiten der Wähler eröffnet den Politikern einen gewissen Handlungsspielraum, der die Durchsetzung von speziellen Regelungen ermöglicht. Hinzu kommt die Tatsache, daß politische Abstimmungen zumeist nur in größeren Zeitabständen $^{2}$ ) erfolgen und die Politiker bis zur nächsten Wahl als Vertreter der Mehrheit eine Art "Monopolstellung" beim Angebot staatlicher Institutionen besitzen. Damit ergeben sich Anreize für Interessengruppen, den politischen Prozeß auch außerhalb von Wahlen durch Lobbying zu beeinflussen. Es werden vor allem diejenigen Interessengruppen zu den Verteilungsgewinnern zählen, von denen sich die Politiker bei der Realisierung ihrer Zielsetzungen Vorteile versprechen ${ }^{3)}$, d.h. zur Erklärung demokratischer Umverteilungsmaßnahmen muß die Interessenlage der Politiker berücksichtigt werden ${ }^{4}$ ).

1) Vgl. Windisch (1984), S. $591 \mathrm{f}$.

2) Zum Aspekt der "zeitlichen Undifferenziertheit" demokratischer Wahlen vgl. Roppel (1979), S. 69. Eine ausführliche Diskussion verschiedener "Unvolikommenheitsfaktoren" findet sich bei Knappe (1980), S. $86 \mathrm{ff}$.

3) Vgl. Johansen (1979), S. 501 ff. bzw. Rose-Ackerman (1980), S. $294 \mathrm{ff}$.

4) Vgl. McCormick/Tollison (1979), S. 5 ff. oder Koford (1987), S. $35 \mathrm{ff}$. 


\subsubsection{Lobbying und Politikerverhalten}

Die Zielsetzung der Bemühungen von Interessengruppen auf der politischen Ebene besteht darin, Harmonie zwischen den Politikerinteressen ${ }^{1}$ ) und den speziellen Gruppeninteressen zu erzielen. Die Gegenleistung der Interessengruppe für die Gewährung von Sonderprivilegien besteht in der Unterstützung bei der Erringung und Erhaltung des politischen Amtes. Neben der Abgabe von Wahlempfehlungen an die Gruppenmitglieder und der finanziellen Förderung von Parteien ist insbesondere die Bereitstellung von Informationen über die Auswirkungen politischer Entscheidungen auf das durch sie vertretene Wählerpotential von Bedeutung. Die Unsicherheit der Politiker über Wählerpräferenzen wird von Interessengruppen durch gezielte Informationspolitik genutzt. Dem Politiker wird deutlich gemacht, welche Stimmenverluste zu erwarten sind, wenn er bestimmte Maßnahmen ergreift bzw. unterläßt. Daraus resultiert die Hypothese ${ }^{2)}$, daß sich Politiker eher an den Forderungen einflußreicher Interessengruppen orientieren werden als an den Wünschen nichtorganisierter Wählerschichten. Unter Wahlgesichtspunkten wird der Politiker versuchen, bei seiner Entscheidung die Differenz zwischen Stimmenzuwächsen und-verlusten zu maximieren ${ }^{3}$ ).

1) Vgl. Congleton (1984), S. $197 \mathrm{ff}$. bzw. (1986), S. $249 \mathrm{ff}$.

2) Vgl. dazu kritisch Wagner (1966), S. $161 \mathrm{ff}$. Als Beispiel für die Berücksichtigung nichtorganisierter Interessen kann die Entwicklung der Altersrenten dienen. Die ökonomischen Interessen der Rentner "sind so spezifisch und ihre Zahl ist so groß, daß sie allein über den Stimmzettel bei Wahlen eine große Macht ausüben, mit deren Hilfe sie ihr Ziel erreichen". v. Weizsäcker (1984), S. 136. Vgl. auch Denzau/ Munger (1983).

3) Vgl. dazu das allgemeine Grundmodell von Peltzman (1976), S. $214 \mathrm{ff} .$, das eine Formalisierung der Argumentation von Stigler (1971) darstellt. Vgl. ebenfalls Posner (1974), S. $335 \mathrm{ff}$. 
Für den "politischen Unternehmer" ist die Aufrechterhaltung allgemeiner Regelungen wenig attraktiv, da diese den Charakter von Kollektivgütern ${ }^{1)}$ besitzen. Diese Eigenschaft von Institutionen läßt ihre Bereitstellung und Durchsetzung im politischen Prozeß kaum erwarten. Gerade die Abstraktheit und Allgemeingültigkeit von fiskalischen bzw. Wettbewerblichen Regelungen sind die Ursache dafür, daß ein Angebot dieser Güter für Politiker wenig Ertrag verspricht ${ }^{2}$ ).

Die Präferenzen der Politiker für gruppenorientierte Sonderregelungen resultieren aus der besonderen Eignung solcher Maßnahmen, Wählerstimmen zu gewinnen. Es ist für Politiker rational, solche Institutionen anzubieten, die gezielt bestimmte Wählerschichten begünstigen. Die rationale Uninformiertheit des Wählers erlaubt es, einzelnen gesellschaftlichen Gruppen oder Wirtschaftszweigen (z.B. Landwirtschaft, Stahlindustrie) Verteilungsvorteile zukommen zu lassen und damit Stimmenzuwächse zu erringen, während die entsprechenden Kosten der Allgemeinheit (Steverzahlern bzw. Konsumenten) auferlegt werden, ohne größere Stimmenverluste befürchten zu müssen ${ }^{3}$. Während die Verteilungsvorteile den Charakter privater Güter haben, stellen die Kosten partikular begünstigender Maßnahmen eher "öffentliche Ungüter" bzw. Schäden dar, die von den betroffenen Individuen kaum wahrgenommen bzw. unterschätzt werden. Gleichzeitig besteht eine Art "Sperrklinkeneffekt"4), d.h. eine Inflexibilität staatlichen Handelns im Hinblick auf eine Verminderung gruppenbezogener Institutionen. Aus politischer Sicht führt die Umkehrung des beschriebenen Prozesses,

1) Vgl. Buchanan (1975), S. $107 \mathrm{ff}$.

2) Vgl. Streit (1986a), S. 99.

3) Vgl. Tietzel (1988b), S. 91.

4) Vgl. Streit (1986a), S. 103. 
nämlich der Abbau gruppenspezifischer Sonderprivilegien zu spürbaren Verlusten bei bestimmten Wählerschichten, denen breitgestreute Entlastungen der Allgemeinheit gegenüberstehen. Auf diese Weise werden kaum Stimmen gewonnen, allerdings sichere Stimmenverluste von seiten der negativ betroffenen Gruppen realisiert.

Die Unsicherheit der Politiker führt zu einer stärkeren Beachtung der Informationen von seiten der interessierten Gruppen, wenn sie bei der speziellen Begünstigung auf die Förderung des Allgemeinwohls verweisen und daher für Wahlzwecke besonders günstig erscheinen. In diesem Zusammenhang eignen sich vor allem staatliche Regulierungseingriffe, um als Ausnahmeregelung von den allgemeinen wettbewerblichen Institutionen Sonderinteressen politisch opportun zu verbergen ${ }^{1)}$. Ungeachtet der proklamierten Zwecke eröffnen diese Maßnahmen spezielle Verteilungsvorteile für eng begrenzte Gruppen und belasten einen großen Teil der Gesellschaft in ihrer Eigenschaft als Konsumenten. Der Unkenntnis der Vorteile einer Aufrechterhaltung von marktlichen Institutionen auf seiten der Konsumenten steht die Kenntnis der Nachteile auf der Produzentenseite gegenüber. Daraus ergibt sich die Schlußfolgerung, daß im Regierungshandeln eine Prädominanz von Produzenteninteressen im Vergleich zu Konsumenteninteressen zum Ausdruck kommt ${ }^{2}$ ). Neben Effizienzargumenten dienen häufig auch meritorische Motive zur Rechtfertigung ${ }^{3}$ )

1) Vgl. Crew/Rowley (1988), S. 57.

2) Wenngleich aufgrund der gestiegenen Umweltschutzinteressen auch Maßnahmen zu Lasten der Produzenten erfolgen, begünstigt die Praxis des Umweltschutzes vor allem bereits etablierte Unternehmen. Die Regelungen richten sich meist auf den Bau never Anlagen und wirken daher als Marktzutrittserschwerung für neue Konkurrenten. Vgl. v. Weizsäcker $(1982)$, S. 342 .

3) Vgl. z.B. Hamm (1987), S. 279 ff. bzw. Windisch (1984), S. 583 oder Schmidt (1988), S. 390. 
staatlicher Sonderregelungen. Bei der Gewährung von Privilegien zugunsten spezieller Gruppen werden meist Instrumente eingesetzt, die sich durch eine größere Unmerklichkeit ${ }^{1}$ auszeichnen. Beispielsweise ist unter Effizienzgesichtspunkten der Einsatz ungebundener Einkommenstransfers zu befürworten. Allerdings würde diese relativ leicht zu identifizierende Umverteilung im politischen Prozeß eine starke Opposition hervorrufen. Aus diesem Grund werden eher Sachtransfers, Steuervergünstigungen bzw. Subventionen angeboten, die aufgrund der hohen Informationsanforderungen einen geringeren politischen Widerstand auslösen, jedoch zu Allokationsverlusten in Form von Mehrbelastungen führen. Diese Institutionen erlauben eine langfristige Sicherung der Verteilungsvorteile und besitzen aufgrund ihrer Unmerklichkeit gute Erfolgsaussichten im politischen Prozeß.

Die politisch opportune Vergabe neuer Vergünstigungen bei Aufrechterhaltung alter Privilegien impliziert eine entsprechend erhöhte Finanzierungsnotwendigkeit. Die Möglichkeiten zur Durchsetzung partikularer Rent-Seeking-Interessen werden durch die faktische Außerkraftsetzung der Regel des materiellen Budgetausgleichs begünstigt. Das Instrument der Staatsverschuldung erlaubt die Erfüllung vermehrter Ansprüche einzelner Gruppen, indem die Verteilung der damit verbundenen Belastungen unmerklich bleibt ${ }^{2}$ ). Die Konsequenz sind weitere Ansprüche von neven und bestehenden Gruppen, da keine wesentliche Opposition erfolgt.

1) Im Gegensatz dazu präferieren Politiker bei Themen, die ein breites Interesse in der öffentlichkeit finden, Maßnahmen mit hoher "Demonstrationswirkung". So wird bei Regelungen zum Umweltschutz meist auf Verbote, Auflagen und Standards zurückgegriffen, wenngleich andere Instrumente (z.B. Vergabe von Umweltzertifikaten) allokativ vorzuziehen wären. Vgl. Tietzel (1988b), S. $90 \mathrm{ff}$.

2) Vgl. Folkers (1986b), S. 385 bzw. auch Rowley/Shughart II/ Tollison (1987), S. $264 \mathrm{ff}$. 
Nach dem Wahlsieg verfügen Politiker über einen diskretionären Handlungsspielraum, innerhalb dessen von den Wählerpräferenzen abweichende Entscheidungen getroffen werden können. Wenngleich gewisse Restriktionen durch die Wiederwahl gegeben sind, wird der Politiker seinen Handlungsspielraum zu Maßnahmen nutzen, die seinen Eigeninteressen entsprechen. Dabei erweist sich das Rent-Seeking privater Interessengruppen als geeignetes Instrument zur Gewinnung von politischem Einfluß für Politiker, die ihrerseits durch Ausnutzung der Forderungen der Interessengruppen ihre persönlichen Interessen zu verwirklichen suchen. Im Gegensatz zur Vorstellung eines staatlichen Leviathan ${ }^{1}$ ), der seine Entscheidungsfreiheit zur vollständigen Ausbeutung der Bürger nutzt, besteht das zentrale Ziel der Politiker in der Sicherung einer "satisfizierenden Finanzmasse zur Realisierung der unterschiedlichen, ihren Eigeninteressen nützlich erscheinenden Gruppenvorteile im Steuer- und Ausgabenbereich"2).

Die Notwendigkeit zur Stimmengewinnung für die Politiker fördert die Entwicklung eines "Vergünstigungsstaates" 3 ), der durch die Berücksichtigung zahlreicher Sonderinteressen gekennzeichnet ist. Die von Wählerstimmen abhängigen Politiker werden den Gruppenforderungen nachkommen und Sondervergünstigungen gewähren, die in aller Regel zu Allokationsverlusten in Form von Mehrbelastungen führen. Allerdings fällt auf, daß nicht systematisch einzelne Gruppen begünstigt werden, sondern daß eine Vielzahl staatlicher Regelungen existiert, die gegensätzliche Gruppeninteressen berücksichtigen.

1) Vgl. Brennan/Buchanan (1980/1988), Kap. 2. Zum Politikerverhalten bei Verfolgung von Eigeninteressen und wiederwahlfördernden Maßnahmen sei auf Barro (1973), S. $19 \mathrm{ff}$. verwiesen. Die aktive Rolle von Politikern beim Rent-Seeking betonen Appelbaum/Katz (1987), S. 685 ff. Und McChesney (1987), S. 1 ff.

2) Folkers (1983a), S. 202.

3) Folkers (1986a), S. 25. 


\subsubsection{Stimmentausch}

Zur Erklärung des Phänomens, daß in demokratischen Systemen zahlreiche staatliche Maßnahmen zugunsten von Minderheiten erfolgen, ist auf den Mechanismus des sogenannten Stimmentauschs ${ }^{1}$ zu verweisen. Diese Variante politischer Einflußnahme eignet sich für Konstellationen, in denen einzelne Gruppen alternativen staatlichen Regelungen ein unterschiedliches Gewicht beimessen. Da in aller Regel über mehrere Fragen gleichzeitig entschieden wird und damit ein mehrdimensionaler Alternativenraum vorliegt, besteht für Gruppen der Anreiz, einer sie leicht benachteiligenden staatlichen Maßnahme zuzustimmen, wenn sie im Gegenzug die Unterstützung durch eine andere Gruppe in der für sie besonders wichtigen Angelegenheit erhalten. Auf diese Weise können Minoritäten sich gegenseitig zu einer ausreichenden Mehrheit für beide Anliegen verhelfen, die bei getrennter Abstimmung jeweils gescheitert wären. Im politischen Prozeß schlagen sich eher die Interessen von Minderheiten mit starken Präferenzen nieder, während die schwächeren Präferenzen der Mehrheit weniger zur Geltung kommen ${ }^{2}$ ).

Die Möglichkeit des Stimmentauschs kann auch zur Erklärung der Berücksichtigung zahlreicher divergierender Partikularinteressen in der repräsentativen Demokratie dienen. Politiker werden sogenannte "Programmpakete" schnüren, in der jede der beteiligten Gruppen ihren spezifischen Interessenstandpunkt gewahrt sieht ${ }^{3)}$. Mittels der Verknüpfung geeignet formulierter Gesetzesvorlagen, von denen jede für sich allein betrachtet nur eine Minderheit interessiert, sichern sich die Politiker die Stimmen der Minderheitsgruppierungen.

1) Zur Problematik des Logrolling vgl. Tullock (1959), S. 571 ff. sowie Buchanan/Tullock (1962), Kap. 10 und Mueller (1979).

2) Vgl. Stigler (1971), S. 12 .

3) Dieser Vorgang wird auch als "impliziter Stimmentausch" bezeichnet. Buchanan/Tullock (1962), S. 134. 
Als Konsequenz dieses Verfahrens ist ein inkonsistentes staatliches Handeln zu erwarten, das sich in einer Vielzahl widersprüchlicher Institutionen zugunsten partikularer Interessen niederschlägt. Auf der Suche nach parlamentarischen Mehrheiten werden nämlich Hilfen für die Landwirtschaft mit Unterstützungen für die Stahlindustrie gebündelt; Fördermittel für sogenannte "zukunftsorientierte Schlüsseltechnologien" werden gleichzeitig mit Erhaltungssubventionen für "alternde" Industrien vergeben; Subventionen für den Zonenrand finden sich neben Sonderprogrammen zugunsten von Ballungsgebieten und Investitionszulagen für traditionelle Kohlereviere werden in einem Zug mit Stromhilfen für andere Regionen beschlossen.

Da man den politischen Wettbewerb als ein Ringen der Politiker um die Stimmen marginaler Gruppen interpretieren kann, ergibt sich für Politiker zusätzlich ein Anreiz, immer neue spezifische Gruppeninteressen aufzuspüren und durch Einbindung in Wahlprogramme zu kollektiven Anliegen zu machen ${ }^{1)}$. Aus der Ausrichtung politischer Entscheidungen an Partikularinteressen ${ }^{2}$ ) ergibt sich eine Kumulation ständig neuer Regelungen, die eine eindeutige Bestimmung der damit verbundenen Verteilungseffekte verhindern und die Informationsgewinnung über das Ergebnis der unterschiedlichen Maßnahmen durch die Wähler erschweren. Mit den zunehmenden Widersprüchlichkeiten eines immer komplexeren Systems staatlicher Institutionen werden weitere Anreize zu Rent-Seeking-Vorhaben interessierter Gruppen geschaffen. Solange die Mitglieder spezifischer Gruppen Verteilungsvorteile erringen und sich keine größere gesellschaftliche Gruppe zu stark benachteiligt sieht, ist das politische system stabil ${ }^{3)}$.

1) Vgl. Blankart (1983), S. 157.

2) Vgl. Schmidt (1966), S. $213 \mathrm{ff}$.

3) Vgl. Z.B. Laux (1984), S. $111 \mathrm{f}$. 


\subsection{Die Interessen der Bürokratie}

Das Rent-Seeking privater Interessengruppen begünstigt das Zustandekommen statlicher Institutionen, die bestimmten Gruppen Verteilungsvorteile garantieren. Die Aufgabe der Bürokratie besteht in der Durchführung der vom Parlament und der Regierung beschlossenen Gesetze. Allerdings werden die Staatsbediensteten ebenfalls eigene Ziele und Interessen verfolgen. Bürokraten besitzen analog den Politikern einen gewissen Handlungsspielraum, innerhalb dessen sie ihren Nutzen maximieren können.

Unter der Annahme, daß das Streben nach Machtbefugnissen, Sicherheit bzw. nach Einkommen positiv mit der Höhe des Budgets $^{1)}$ korreliert, ist die Bürokratie an einer Ausdehnung staatlicher Gesetze und Maßnahmen interessiert ${ }^{2}$. Das RentSeeking privater Interessengruppen fördert das Expansionsstreben der Bürokraten. Der politische Einfluß einzelner Gruppen wird noch verstärkt, wenn ihre Vertreter in Positionen der Ministerialbürokratie gelangen und direkt auf die Ausgestaltung staatlicher Institutionen einwirken können ${ }^{3)}$. Der Informationsvorsprung der Bürokratie über die Besonderheiten einzelner Institutionen eröffnet Möglichkeiten zur Realisierung von Produzentenrenten innerhalb des staatichen Sektors, die mit erhöhtem Einfluß auf Art und Ausführung staatlich determinierter Institutionen ansteigen ${ }^{4}$.

1) Vgl. Niskanen (1971) bzw. Roppel (1979), S. 19 ff.

2) Vgl. Borcherding (1977), S. $60 \mathrm{ff}$.

3) Zur Einflußnahme der Bürokratie auf das Zustandekommen never Vorlagen bzw. der Novellierung bestehender Gesetze vgl. Schulze-Fielitz (1986), S. 71 ff.

4) Vgl, auch Flowers/Stroup (1979), S. 473 ff. und Faith (1980), S. $332 \mathrm{ff}$. bzw. Findlay/Wellisz (1984), S. $97 \mathrm{ff}$. sowie Benson/Mitchell (1988), S. $5 \mathrm{ff}$. 
Zur Durchsetzung ihrer Ziele wird die Bürokratie nur unzureichende Informationen über die tatsächliche Produktions- bzw. Kostenfunktion der Leistungserstellung vermitteln. Dieses Verhalten wird wiederum durch die Vielfalt von Maßnahmen in nahezu allen Bereichen staatlichen Handelns gefördert. Mit zunehmendem Budgetumfang und steigender Regulierungsdichte werden entsprechende Kontrollmöglichkeiten immer aufwendiger und in ihrer Wirkung eingeschränkt.

Unterstellt man, daß die öffentliche Verwaltung eine Monopolstellung gegenüber dem Parlament besitzt und als Optionsfixierer auftreten kann, ist eine Abschöpfung der gesamten Konsumentenrente der staatlichen Leistungen möglich. Neben den Vorteilen aus einem ineffizient hohen Budget ${ }^{1)}$, ergeben sich zusätzliche Renten für die Bürokratie bei Abweichung von der Produktion zu Minimalkosten. Außer allokativen sind folglich auch produktionstechnische Ineffizienzen zu erwarten. Darüber hinaus werden die Bürokraten bei der Ausübung ihres Wahlrechts entsprechend ihrer Ziele eher Ausgabensteigerungen befürworten und Budgetkürzungen ablehnen ${ }^{2}$ ). Auf diese Weise werden die Beschäftigten der staatlichen Bürokratie automatisch zu Förderern von Rent-Seeking-Vorhaben, die eine Vergrößerung des Budgets durch gruppenspezifisch begünstigende Ausgabenkomponenten anstreben. Die Politiker werden die Wünsche der Bürokratie nicht übergehen, um die Unterstützung der Beschäftigten der Staatsbürokratie zu erhalten. Um Informationen zu gewinnen und die politisch opportune Ausübung des diskretionären Ermessensspielraums von seiten der Bürokratie zu sichern, werden die Politiker ihre Beziehungen zur Verwaltung pflegen und Interessenharmonie anstreben.

1) Vgl. Migué/Bélanger (1974), S. 27 ff. bzw. Orzechowski (1977), S. $229 \mathrm{ff}$. sowie Romer/Rosenthal (1978), S. $27 \mathrm{ff}$. und $(1979)$, S. $563 \mathrm{ff}$. bzw. (1982), S. $556 \mathrm{f}$.

2) Vgl. Bush/Denzau (1977), S. $90 \mathrm{ff}$. 


\section{Interessengruppen und Zunahme der Staatstätigkeit}

Die Diskussion der Bedeutung organisierter Gruppeninteressen für die Ergebnisse des politischen Entscheidungsprozesses in der Demokratie wirft die Frage auf, in welcher Weise der Einfluß von Interessengruppen eine Zunahme der Staatstätigkeit bewirkt. Damit wird dem Aspekt Rechnung getragen, daß staatliche Institutionen auch die Nachfrage nach staatlichen Leistungen beeinflussen ${ }^{1)}$. Nach der Darstellung des Referenzmodells werden zunächst die Effekte einer gruppenspezifisch begünstigenden Ausgestaltung des Steuersystems untersucht. Im zweiten Schritt wird auf die Implikationen einer an Gruppeninteressen ausgerichteten Ausgabenstruktur eingegangen ${ }^{2}$ ).

\subsection{Das Grundmodell}

Gegenstand des Grundmodells ist die Analyse der Beziehungen zwischen der Höhe der gewünschten Bereitstellung eines rein öffentlichen Gutes und dem entsprechenden Finanzierungsbeitrag für die jeweilige Interessengruppe. Zu diesem Zweck wird zunächst die Konstellation ohne Einflußnahme von seiten einer bestimmten organisierten Interessenvertretung betrachtet. Ausgangspunkt der Analyse sei die Annahme, daß sich die Interessengruppen aus identischen Individuen zusammensetzen ${ }^{3)}$.

1) Vgl. dazu Übersicht 2 auf S. 142 dieser Arbeit.

2) Im Rahmen des Medianwähler-Modells wird hingegen explizit von gegebenen Steuerpreisen und einem eindimensionalen Alternativenraum ausgegangen, d.h. es wird nur der Umfang, jedoch nicht die Zusammensetzung des Budgets untersucht.

3) Gilt beim gruppeninternen Entscheidungsprozeß die Einstimmigkeitsregel, kann die Analyse auf die Betrachtung eines repräsentativen Gruppenmitgliedes beschränkt werden. 
Die individuelle Nutzenfunktion eines repräsentativen Mitgliedes einer beliebigen Gruppe i wird durch

$$
u^{i}=u^{i}\left(G, x^{i}\right)
$$

wiedergegeben, wobei $G$ die Menge eines rein öffentlichen Gutes und $x^{i}$ die Menge eines privaten Gutes bezeichnet. Während das rein öffentliche Gut nach der polaren Definition von Samuelson ${ }^{1}$ allen Gruppen in gleichem Umfang zur Verfügung steht ${ }^{2}$, differieren die gruppenspezifischen Mengen des privaten Gutes.

Gemäß

(VI.2) $U_{G}^{i}=\frac{\partial U^{i}}{\partial G}>0 \quad b z w . \quad U_{X}^{i}=\frac{\partial U^{i}}{\partial x^{i}}>0$

und

$$
U_{G G}^{i}=\frac{\partial^{2} U^{i}}{\partial G^{2}}<0 \quad \text { bzw. } \quad U_{X X}^{i}=\frac{\partial^{2} U^{i}}{\partial x^{i^{2}}}<0
$$

werden positive Grenznutzen beim Konsum beider Güter sowie abnehmende Nutzenzuwächse unterstellt. Die Entscheidung des Individuums wird bei gegebenem Preis $\left(p_{X}\right)$ des privaten Gutes von den Kosten der Bereitstellung des öffentlichen Gutes und dem durch das Steuersystem festgelegten individuellen Steuerpreis $\left(t^{i}\right)$ bestimmt. Zur Vereinfachung wird angenommen, daß sich der

1) Vgl. Samuelson (1954), S. $386 \mathrm{ff}$. bzw. (1955), S. $3.50 \mathrm{ff}$.

2) Allerdings differiert die individuelle Bewertung des'öffentlichen Gutes. 
individuelle Steuerpreis als prozentualer Anteil an den Kosten des öffentlichen Gutes darstellt, so daß

(VI.4) $\quad \sum_{i} t^{i}=1 \quad$ für $i=1, \ldots, n$

gilt. Das Individuum sieht sich damit der Budgetrestriktion (VI.5) $\quad Y^{i}=p_{X} x^{i}+t^{i} G$

gegenüber, wobei $Y^{i}$ das Einkommen wiedergibt. Zur Maximierung der individuellen Nutzenfunktion unter Berücksichtigung der Nebenbedingung (VI.5) wird die Lagrange-Funktion

(VI.6)

$$
L=u^{i}\left(G, x^{i}\right)+\mu\left(y^{i}-t^{i} G-p_{X} x^{i}\right)
$$

gebildet, woraus sich die notwendigen Bedingungen als

(VI.7) $\quad U_{G}^{i}-\mu t^{i}=0$

bzw.

(VI.8) $\quad U_{X}^{i}-\mu p_{X}=0$

ergeben. Wählt man $X$ als numéraire-Gut und setzt $P_{X}$ gleich eins, stellt sich die marginale Rate der Substitution zwischen beiden Gütern in Form von 1 )

(VI.9) $\quad \frac{U_{G}^{i}}{U_{X}^{i}}=t^{i}$

dar.

1) Vgl.z.B. Breton (1974), S. 60 oder auch Mueller/Murrell (1985), S. 15 . 
Die hinreichende Bedingung ist durch

(VI.10) $\quad U_{G G}^{i}-2 t^{i} U_{G X}^{i}+t^{i^{2}} U_{X X}^{i}<0$

gegeben, wobei für die gemischte partielle Ableitung

(VI.11)

$$
\frac{\partial}{\partial x^{i}}\left(\frac{\partial U^{i}}{\partial G}\right)>0
$$

und

(VI.12) $\quad U_{X G}^{i}=U_{G X}^{i}$

gilt ${ }^{1)}$

Wenn die Bedingung (VI.9) für die Gruppenmitglieder der einzelnen Interessengruppen erfüllt ist, liegt ein sogenanntes Lindahl-Gleichgewicht ${ }^{2}$ ) vor. Diese Steuerstruktur impliziert eine effiziente Bereitstellung des öffentlichen Gutes, da der individuelle Steuerpreis jeweils der marginalen Austauschrate zwischen öffentlichem und privatem Gut entspricht ${ }^{3}$ ). Die Individuen werden sich einstimmig für die Bereitstellung der effizienten Menge $G^{*}$ entscheiden. Auf der Grundlage dieses Referenzmodells ${ }^{4}$ ) werden die Wirkungen staatlicher Vergünstigungen auf der Steuer-bzw. Ausgabenseite des Budgets im Hinblick auf eine Zunahme der Staatstätigkeit analysiert.

1) Vgl. Henderson/Quandt (1983), S. $401 \mathrm{ff}$.

2) Vgl. Lindahl (1919). Eine zusammenfassende Darstellung findet sich bei Johansen (1963), S. 346 ff. bzw. (1965), S. $129 \mathrm{ff}$.

3) Vgl. Breton (1974), S. $64 \mathrm{f}$.

4) Vgl. Mueller/Murrell (1985), S. 15. 


\subsection{Die Effekte einer begünstigenden Steuerstruktur}

Eine veränderte Steuerstruktur wird zu divergierenden Mengennachfragen nach dem öffentlichen Gut führen, so daß die Art des Entscheidungsverfahrens das kollektive Ergebnis bestimmt. Bei Abstimmung mit einfacher Mehrheit wird der Medianwähler die entscheidende Position einnehmen. Jede Veränderung des Besteverungsverfahrens mit differenzierten Steuerpreisen kann andere Gruppen in die Medianposition bringen und damit den Umfang der nachgefragten Menge an öffentlichen Leistungen beeinflussen. Die begünstigende Ausgestaltung der Steuerstruktur mit dem wahlentscheidenden Einfluß einzelner Wählergruppen induziert gewisse Anreize zur Ausdehnung der Staatstätigkeit.

Verteilungskoalitionen, die Stevervorteile für ihre Mitglieder im demokratischen Mehrheitssystem beschließen, werden infolge der verminderten Steuerpreise eine höhere Bereitstellung öffentlicher Güter wünschen. Als Konsequenz der begünstigenden Steverstruktur werden die erfolgreichen Gruppierungen - im Vergleich zum Ausgabenniveau unter Gültigkeit der Einstimmigkeitsregel - überhöhte Mengen der von ihnen gewünschten öffentlichen Güter nachfragen ${ }^{1)}$, während bei den Wahlverlierern die Grenzkosten die marginale Bewertung der öffentlichen Güter übersteigen. Aus diesem Grund resultiert eine allokativ überhöhte Staatstätigkeit. Kommen die staatlichen Leistungen vorwiegend speziellen Gruppen zugute, ist darüber hinaus eine ineffiziente Struktur staatlicher Aktivitäten zu erwarten ${ }^{2}$.

1) In diesem Zusammenhang ist wiederum auf das Verfahren des "Logrolling" zwischen den Gruppen zu verweisen. Die Ursache für die ineffizient hohe Staatstätigkeit liegt darin, daß jede Interessenkoalition bei ihrem Marginalkalkül lediglich die von ihr zu tragenden Grenzkosten berücksichtigt. Vgl. insbesondere Buchanan/Tullock (1962), S. $164 \mathrm{ff}$.

2) Vgl. Folkers $(1986 a)$, S. 16. 
Selbst wenn das bei gegebener Besteuerung gewünschte Ausmaß an öffentlichen Gütern gemäß der Austauschbeziehung (VI.9) bereitgestellt wird, bestehen Anreize, durch Einflußnahme auf den politischen Prozeß eine Verminderung des Steuerpreises anzustreben ${ }^{1)}$. Aufgrund der fehlenden direkten Beziehung von Stevern und Gegenleistung kann die Steuerverpflichtung und damit die Kostenverteilung statlicher Leistungen ohne Rückwirkungen auf die Budgetentscheidungen geändert werden ${ }^{2}$ ). Beispielsweise ist bei vollständiger Vermeidung der individuellen Steuerpflicht und erhöhter Besteverung anderer gesellschaftlicher Gruppen bei Aufrechterhaltung eines bestimmten statlichen Leistungsniveaus eine individuelle Nutzensteigerung durch erhöhten Konsum privater Güter möglich.

Bei dieser individuellen Interessenkonstellation werden "ähnlich wie bei marktmäßigen überwälzungsprozessen auch allokative Ineffizienzen in Kauf genommen, wenn die Sicherung der unmittelbar begünstigenden, individuell teilbaren Distributionsvorteile dies nahelegt" ${ }^{3)}$. Der Umfang der Rent-SeekingAktivitäten hängt vom Ausmaß der potentiellen Renten einer gruppenspezifisch vorteilhaften Veränderung der Fiskalstruktur ab, die sich in gleicher Weise auf der Ausgabenseite des Budgets realisieren lassen.

1) Wenngleich die durch den Medianwähler determinierte Menge an öffentlichen Gütern ein kollektives Gleichgewicht impliziert, ist es individuell vorteilhaft, sich der damit verbundenen Steververpflichtung zu entziehen. Vgl. hierzu Buchanan/Flowers (1969), S. $349 \mathrm{f}$. bzw. in ähnlicher Weise Jackson (1971), S. 95 und Folkers (1983a), S. 199.

2) Vgl. Folkers (1983a), S. $193 \mathrm{f}$.

3) Folkers (1983a), S. 194. 


\subsection{Die Effekte einer begünstigenden Ausgabenstruktur}

Auf der Ausgabenseite ergeben sich Anreize für Interessengruppen, speziell begünstigende Güter kollektiv bereitstellen zu lassen ${ }^{1)}$. Interessengruppen versuchen, vorteilhafte Ausgabenkomponenten durchzusetzen und die Struktur des staatlichen Leistungsangebotes $z u$ verändern. Bei der folgenden Analyse wird unterstellt, daß die Steverstruktur des Grundmodells aufrechterhalten bleibt. Auf dieser Grundlage wird zunächst das Verhalten der begünstigten Gruppe bei der Frage über den Umfang der kollektiven Bereitstellung eines Gutes mit gruppenspezifischen Eigenschaften betrachtet. Im zweiten Schritt werden die daraus resultierenden Konsequenzen für die Höhe der gesamten staatlichen Ausgaben untersucht. In analoger Vorgehensweise wird anschließend auf das Verhalten von oppositionellen Gruppen eingegangen.

\subsubsection{Das Verhalten der begünstigten Gruppe}

Ausgangspunkt der Überlegung ist die Vorstellung, daß einer Interessengruppe $i$ die Realisierung eines ungebundenen monetären Transfers $Z$ gelingt. Die Nutzenfunktion eines repräsentativen Individuums dieser Gruppe stellt sich gemäß

(VI.13) $\quad U^{i}=U^{i}\left(G, x^{i}+Z\right)$

dar, d.h. es resultiert eine Steigerung der Konsummöglichkeiten des privaten Gutes ${ }^{2}$ ).

1) Vgl. Spann (1974), S. $63 \mathrm{f}$.

2) "One obvious form of targeted good is a direct cash transfer, allowing the interest group's members to increase their consumption of private goods." Mueller/Murrell $(1985)$, S. 16 . 
In aller Regel haben staatliche Leistungen den Charakter von Mischgütern. "These public expenditures, although having an obvious private-good characteristic for the recipients, also have spillover effects that make them partially of public-good nature"1). Da Sachtransfers eine bessere Verschleierung der eigentlichen Verteilungsintentionen erlauben und mit dem Hinweis auf die Erfüllung übergeordneter gesellschaftlicher Ziele gerechtfertigt werden können, ist eine geringere Opposition als bei direkten Einkommenstransfers zu erwarten ${ }^{2}$ ).

Die Eigenschaften von Mischgütern werden in der modifizierten Nutzenfunktion eines Individuums der unmittelbar begünstigten Gruppe i

(VI.14) $\quad U^{i}=U^{i}\left[G+\theta Z, X^{i}+(1-\theta) Z\right]$

berücksichtigt. Der Parameter $\theta$ bezeichnet den Anteil des Gutes $Z$, der kollektiven Nutzen stiftet, während der private, ausschließlich die Gruppe begünstigende Anteil durch $(1-\theta)$ symbolisiert wird. Aufgrund von

$0 \leqq \theta \leqq 1$

kann damit sowohl der Fall von rein gruppenspezifischen Gütern $\left.(\theta=0)^{3}\right)$ als auch die Konstellation von Leistungen mit rein öffentlichem Gutscharakter $(\theta=1)$ erfaßt werden.

1) Mueller/Murrell (1985), S. 16.

2) So werden die begünstigten Gruppen für die Ausweitung von Programmen des sozialen Wohnungsbaus, für weitere Aktivitäten im Umweltschutzbereich bzw. Bildungsbereich etc. plädieren. Vgl. Z.B. Pommerehne/Kirchgässner (1988), S. 230.

3) In diesem Fall reduziert sich (VI.14) wiederum zu (VI.13). 
Bei der Maximierung der individuellen Nutzenfunktion sind als Nebenbedingungen die modifizierte Budgetrestriktion ${ }^{1}$ )

(VI.16) $\quad Y^{i}=t^{i}(G+Z)+x^{i}$

sowie eine bestimmte politisch festgelegte Obergrenze m für die maximal mögliche Bereitstellung des Mischgutes in Form von

(VI.17) $\quad Z \leqq m$

zu berücksichtigen ${ }^{2}$ ). Durch partielle Differentiation der Lagrange-Funktion

(VI.18)

$$
L=U^{i}\left[G+\theta Z, x^{i}(1-\theta) Z\right]
$$

$$
+\mu\left[Y^{i}-t^{i}(G+z)-x^{i}\right]+n[m-z]
$$

nach G bzw. X resultieren wiederum (VI.7) bzw. (VI.8), während die partielle Ableitung nach $Z$ als

(VI.19) $\quad \frac{\partial L}{\partial Z}=\theta U_{G}^{i}+(1-\theta) U_{X}^{i}-\mu t^{i}-\eta=0$

ermittelt wird.

1) Vgl. Mueller/Murrell (1985), S. 17.

2) Diese Nebenbedingung begrenzt den Handlungsspielraum für die Gewährung von Privilegien und "may be interpreted as the government's mandate from last election and/or as that elbow-room of the government which, .... does not jeopardize its survival in the forthcoming election". Pethig (1988), S. 264. 
Nach geeigneter Substitution läßt sich

(VI.20)

$$
(1-\theta) \frac{U_{G}^{i}}{t^{i}}+(\theta-1) U_{G}^{i}=n
$$

und damit

(VI.21)

$$
(1-\theta) U_{G}^{i}\left(\frac{1}{t^{i}}-1\right)=n
$$

gewinnen. Aufgrund von
(VI.22)
$n>0$

kann unter Verwendung der Kuhn-Tucker-Bedingungen

(VI.23) $\quad Z=m$

bestimmt werden ${ }^{1}$ ).

Als Konsequenz dieser Argumentation ist festzuhalten, daß die betrachtete Interessengruppe bei der Frage, in welchem Umfang eine kollektive Bereitstellung des gruppenspezifischen Gutes $Z$ erfolgen soll, das maximal mögliche Niveau fordern wird ${ }^{2}$. Im weiteren werden die daraus resultierenden Wirkungen für die gesamten statichen Ausgaben $(G+Z)$ untersucht.

1) Eine zusammenfassende Darstellung und Interpretation der Kuhn-Tucker-Bedingungen zur Lösung von Optimierungsaufgaben findet sich bei Neumann (1975), S. $234 \mathrm{ff}$.

2) Vgl. Mueller/Murrell (1985), S. 33. 
Zu diesem Zweck wird die Nutzenfunktion ${ }^{1}$ )

$$
\text { (VI.24) } \quad U^{i}=U^{i}\left[G+\theta m, x^{i}+(1-\theta) m\right]
$$

maximiert und

$$
\text { (VI.25) } \quad U_{G}^{i}=t^{i} U_{x}^{i}
$$

abgeleitet. Durch totale Differentiation ergibt sich

(VI.26)

$$
U_{G G}^{i}(d G+\theta d m)+U_{G X}^{i}\left[d X^{i}+(1-\theta) d m\right]=
$$

$$
t^{i} U_{G X}^{i}(d G+\theta d m)+t^{i} U_{X X}^{i}\left[d X^{i}+(1-\theta) d m\right] .
$$

Unter Verwendung von

(VI.27) $\quad \frac{d X^{i}}{d m}=-t^{i}\left(\frac{d G}{d m}+1\right)$

wird nach Substitution und geeigneten Umformungen

(VI.28) $\frac{d G}{d m}=-1+(1-\theta)\left[\frac{U_{G G}^{i}-U_{G X}^{i}\left(1+t^{i}\right)+t^{i} U_{X X}^{i}}{U_{G G}^{i}-2 t^{i} U_{G X}^{i}+t^{i^{2}} u_{X X}^{i}}\right]$

ermittelt.

1) Vgl. Mueller/Murrell (1985), S. 33. 
Aufgrund der Bedingung zweiter Ordnung (VI.10) besitzt der Nenner ein negatives Vorzeichen, so daß der Gesamteffekt vom Vorzeichen des Zählers abhängt. Unter Berücksichtigung der Eigenschaften (VI.2) und (VI.3) bzW. (VI.11) weist der Zähler ebenfalls ein negatives Vorzeichen auf, womit

(VI.29) $\quad \frac{d G}{d m}>-1$

folgt. D.h. ein marginaler Anstieg der Obergrenze für das Gut $Z$ impliziert zwar eine Reduktion des rein öffentlichen Gutes $G$, allerdings in verringertem Ausmaß. Daraus ergibt sich die Schlußfolgerung, daß für die Gesamtausgaben

(VI. 30)

$$
\frac{d(G+m)}{d m}>0
$$

gelten muß. Je höher die Obergrenze $m$ für das Mischgut festgelegt wird, desto höher ist der Umfang der Staatsausgaben. Auf diese Weise wird eine Interessengruppe, die eine Aufnahme eines Gutes mit gruppenspezifisch begünstigenden Eigenschaften in das staatliche Leistungsangebot durchsetzt, zur Ausdehnung der Staatstätigkeit beitragen ${ }^{1}$ ).

1) Vgl. Mueller/Murrell (1985), S. 17. 


\subsubsection{Das Verhalten der oppositionellen Gruppe}

Die Rent-Seeking-Aktivitäten der begünstigten Interessengruppe induzieren positive Externalitäten ${ }^{1)}$ für andere gesellschaftliche Gruppen. Bei der Analyse des Verhaltens dieser Gruppen wird zunächst wieder untersucht, ob bzw. in welchem Umfang diese Gruppen eine Bereitstellung von spezifischen Gütern zugunsten der Interessengruppe i befürworten. Zu diesem Zweck wird ein repräsentatives Mitglied der oppositionellen Gruppe j betrachtet, dessen individuelle Nutzenfunktion sich als

(VI.31) $\quad U^{j}=U^{j}\left(G+\theta Z, x^{j}\right)$

darstellt. D.h. das Gut $Z$ findet lediglich über die externen Effekte infolge der Kollektivguteigenschaft Berücksichtigung. Bei der Maximierung dieser Nutzenfunktion sind als Nebenbedingungen die Budgetrestriktion

(VI.32) $\quad \mathrm{y}^{j}=t^{j}(G+\theta Z)+x^{j}$

sowie der politisch mögliche Spielraum für die Bereitstellung des Mischgutes

(VI.33) $0 \leqq Z \leqq m$

zu beachten ${ }^{2}$ ).

1) Vgl. Lindbeck (1985), S. $309 \mathrm{ff}$. Oder Mueller (1987), S. 132 .

2) Vgl. Mueller/Murrell (1985), S. 33. 
Aus der Lagrange-Funktion

(VI.34)

$$
L=U^{j}\left[G+\theta z, x^{j}\right]+\mu\left[y^{j}-t^{j}(G+Z)-x^{j}\right]
$$$$
+n[m-z]
$$

wird die partielle Ableitung nach $Z$ als

(VI.35) $\quad \frac{\partial L}{\partial Z}=\theta U_{G}^{j}-\mu t^{j}-\eta=0$

ermittelt. Umgeformt ergibt sich

(VI.36)

$$
(\theta-1) U_{G}^{j}=\eta
$$

woraus

(VI.37)

$\eta<0$

folgt. Als Konsequenz dieser Überlegung muß

(VI.38)

$$
Z=m=0
$$

gelten ${ }^{1)}$. Während die Opposition für den vollständigen Verzicht auf das betreffende Gut plädiert, wünscht die begünstigte Gruppe eine Bereitstellung im höchst möglichen Umfang. Somit ist immer dann mit politischem Widerstand zu rechnen, wenn die Einführung von Gütern mit gruppenspezifisch begünstigenden Eigenschaften in das staatliche Leistungsangebot zur Diskussion steht.

1) Zur Ableitung dieser Schlußfolgerung wird wieder auf die Kuhn-Tucker-Bedingungen zurückgegriffen. 
Für die Entwicklung der Gesamtausgaben ist bedeutsam, wie sich die Interessengruppe $j$ verhäl, wenn der anderen Gruppe die Durchsetzung der vorteilhaften Ausgabenkomponente gelungen ist. Damit ist das quasi-private Gut Bestandteil des öffentlichen Leistungsangebotes, so daß

(VI.39)

$$
z=m>0
$$

erfüllt ist.

Ausgehend von

(VI.40) $\quad u^{j}=U^{j}\left(G+\theta m, x^{j}\right)$

wird durch totale Differentiation von (VI.7)

(VI.41)

$$
U_{G G}^{j}(d G+\theta d m)+U_{G X}^{j} d X^{j}=
$$

$$
t^{j} U_{G X}^{j}(d G+\theta d m)+t^{j} U_{X X}^{j} d X^{j}
$$

und nach geeigneten Umformungen

$$
(V I .42) \quad \frac{d G}{d m}=-1+(1-\theta)\left[\frac{U_{G G}^{j}-t^{j} U_{G X}^{j}}{U_{G G}^{j}-2 t^{j} U_{G X}^{j}+t^{j} U_{X X}^{j}}\right]
$$

ermittelt. Da sowohl der Zähler als auch der Nenner des Klammerausdrucks ein negatives Vorzeichen aufweisen, resultiert insgesamt ein positives Vorzeichen ${ }^{1)}$.

1) Zur analytischen Herleitung sei auf Mueller/Murrell $(1985)$, S. $33 \mathrm{f}$. verwiesen. 
Daraus lassen sich die zu (VI.29) bzW. (VI.30) äquivalenten Beziehungen

(VI.43) $\frac{d G}{d m}>-1$

bzw.

$(V I .44) \quad \frac{d(G+m)}{d m}>0$

gewinnen.

Das wesentliche Ergebnis dieser Analyse besteht in der Erkenntnis, daß die Interessengruppe $j$ ebenfalls einen Anstieg der Gesamtausgaben wünscht. Gelingt einer Gruppe die Aufnahme eines "private or quasi-private good into the public budget, all members of society will agree that there should be an increase in total government expenditure over what it would have been... without the new good"1).

Zur Interpretation dieses Resultates wird davon ausgegangen, daß in der ursprünglichen Konstellation die Bedingung (VI.9) erfült ist. Mit der Bereitstellung von $m$ Einheiten des Gutes $Z$ ergibt sich bei unveränderter Höhe der Gesamtausgaben eine Reduktion von G. Daraus resultiert eine Veränderung der individuellen Austauschrate zwischen öffentlichem und privatem Gut. Für jedes Mitglied der Interessengruppe $j$ vermindert sich der Konsum des rein öffentlichen Gutes um (1 - $\theta)$ m Einheiten, während der Konsum privater Güter im bisherigen Umfang aufrechterhalten bleibt.

1) Mueller/Murrell (1985), S. 17. 
Aus diesem Grund wird nach Einführung von $Z$

$$
\frac{U_{G}^{j}}{U_{X}^{j}}>t^{j}
$$

gelten ${ }^{1)}$. Daraus folgt, daß die Gruppenmitglieder private Güter aufgeben werden, um die aus der Bereitstellung des gruppenspezifischen Gutes $Z$ resultierende Verminderung des rein öffentlichen Gutes zu kompensieren ${ }^{2}$.

\subsection{Interpretation und Kritik der Ergebnisse}

Als Konsequenz dieser Überlegungen ergibt sich, daß eine relative Ausweitung des öffentlichen Sektors nicht aus einer Zunahme der Anzahl von Interessengruppen resultiert, sondern aus dem Rent-Seeking-Verhalten derjenigen Gruppen, deren Aktivitäten positive externe Effekte für andere Gruppen bewirken. Interessengruppen, die ihre Ziele mit der Wahrung übergeordneter gesellschaftlicher Ziele (z.B. Förderung des Schul- und Bildungswesens bzw. des Gesundheitswesens) oder mit Effizienzargumenten ( $z . B$. Verbesserung der Infrastruktur) begründen können, werden eher die Zustimmung anderer Gruppen gewinnen und politische Unterstützung erhalten. Das wesentliche Resultat der Analyse besteht in der Erkenntnis, daß der öffentliche Sektor unter Berücksichtigung der Einflußnahme von seiten interessierter Gruppen einen größeren Umfang aufweist als im Referenzmodell mit passiven Interessengruppen.

1) Vgl. Mueller/Murrell (1985), S. 17 .

2) Dabei ist allerdings die bereits erwähnte Kritik an der Vorstellung eines individuellen Gleichgewichts bei Erfüllung von Bedingung (VI.9) zu berücksichtigen. 
Da eine Konkretisierung des theoretischen Konzepts einer optimalen Staatsquote nicht möglich ist, erlaubt der dargestellte Zusammenhang allerdings keine Aussage darüber, ob zwangsläufig eine systematische Entwicklung in Richtung auf einen "zu hohen" Staatsanteil vorliegt. Wenngleich das Niveau der Staatstätigkeit durchaus auch in bestimmten Perioden zurückgehen kann, wird mit der Durchsetzung gruppenspezifischer Ausgabenkomponenten ein im Vergleich zum Lindahl-Gleichgewicht überhöhtes staatliches Ausgabenvolumen realisiert, d.h. der vorgestellte theoretische Ansatz "predicts excessive levels of government expenditure not necessarily excessive growth in the size of government"1). Bei der komparativ-statischen Vorgehensweise beschränkt sich die Analyse auf die vergleichende Beurteilung des Gleichgewichtszustands vor und nach der Einflußnahme von seiten der Interessengruppen. Für weitergehende Aussagen müssen jedoch auch die Effekte des zum neven Gleichgewicht führenden Entwicklungsprozesses einbezogen und im Rahmen einer dynamischen Betrachtungsweise berücksichtigt werden. Trotz dieser Einschränkungen stellen die aufgezeigten Beziehungen einen Ansatzpunkt zur Erklärung des Umfangs staatlicher Ausgabentätigkeit infolge der Rent-Seeking-Aktivitäten organisierter Gruppeninteressen dar. Die Ausdehnung des öffentlichen Sektors ist das Ergebnis der erfolgreichen Durchsetzung distributiv motivierter staatlicher Institutionen, die ihrerseits die Wirkungsweise der marktlichen Institutionen beeinträchtigen können ${ }^{2)}$.

1) Mueller/Murrell (1985), S. 20. Zu empirischen Analysen vgl. Murrell (1984), S. $151 \mathrm{ff}$. bzw. Schneider (1985), S. $34 \mathrm{ff}$. und Mueller/Murrell (1986), S. $125 \mathrm{ff}$.

2) Vgl. Olson (1982) bzw. Colander/0lson (1984), S. 120 ff. Empirisch gestützt wird diese Hypothese durch die Untersuchungen von Friedland/Sanders (1985), S. $421 \mathrm{ff}$. und Vedder/Gallaway (1986), S. 93 ff. sowie McCallum/Blais (1987), S. 3 ff. Zur Gegenposition sei auf Weede (1984), S. $349 \mathrm{ff}$. verwiesen. Vgl. auch Pryor (1984), S. $158 \mathrm{ff}$. 


\section{Zusammenfassung}

In demokratischen Staatswesen repräsentieren Parteien, Interessengruppen und die Bürokratie einflußreiche politische Institutionen, die beim Zustandekommen kollektiver Entscheidungen zusammenwirken. Interessengruppen werden als Nachfrager nach staatlichen Institutionen interpretiert, die ihre spezifischen Gruppeninteressen durchzusetzen suchen. Politiker bzw. Bürokraten werden als Anbieter staatlicher Regelungen begriffen, die auf die Forderungen organisierter Gruppen eingehen, um Unterstützung bei der Verfolgung ihrer individuellen Zielsetzungen zu erlangen.

Die Analyse der Wirkungsweise der einfachen Mehrheitsregel führt zu dem Ergebnis, daß die Unvollkommenheiten des Wahlprozesses und insbesondere die "rationale Uninformiertheit" der Wähler den Politikern einen diskretionären Handlungsspielraum eröffnen, der zur Verabschiedung von gruppenspezifisch begünstigenden Maßnahmen genutzt werden kann. Damit werden Anreize für Interessengruppen induziert, durch eine gezielte Informationsbeeinflussung von Wählern und Politikern die Realisierung weiterer Privilegien und Sonderregelungen zu bewirken. Die Eigengesetzlichkeit des politischen Wettbewerbs stimuliert die Zunahme solcher Regelungen. Das Streben der Politiker nach Erhaltung und Ausbau politischer Macht sowie der Partizipation an den damit verbundenen Vorteilen erweist sich als Triebfeder für die Berücksichtigung von vielfältigen Sonderinteressen. Um bestimmte Wählerschichten zu erhalten bzw. zu gewinnen, werden sich Politiker für eine interessenbezogene Ausrichtung von staatlichen Regelungen entscheiden. Dieses Verhalten begünstigt die Entwicklung zu einem von Gruppeninteressen abhängigen Vergünstigungsstaat, der einzelnen Gruppen dauerhafte Privilegien verschafft. 
Die zunehmende Interventionsbereitschaft erfordert einen ebenfalls expandierenden Verwaltungsapparat. Damit werden auch die Interessen der Bürokraten gefördert, die bei der Ausführung der vielen Maßnahmen ihren politischen Einfluß steigern und ihre individuellen Zielsetzungen verfolgen können.

Die begünstigende Ausgestaltung staatlicher Institutionen führt neben privaten Anpassungsreaktionen auch zu Reaktionen im Hinblick auf die Nachfrage nach staatlichen Leistungen. Gelingt einer Interessengruppe die Realisierung einer vorteilhaften Steuerstruktur, kann aufgrund des gesunkenen Beitrags der Gruppenmitglieder zur Finanzierung von öffentlichen Gütern eine Erhöhung der Staatstätigkeit induziert werden. Darüber hinaus resultieren weitere Vorteile, wenn statliche Leistungen durchgesetzt werden, die vorwiegend dieser speziellen Gruppe zugute kommen. Interessengruppen werden daher versuchen, die Bereitstellung privater Güter durch politischen Beschluß zu kollektiven Anliegen zu machen. Da ein Teil der Kosten für diese staatlich bereitgestellten Güter mit gruppenspezifischen Eigenschaften von anderen Steverzahlergruppen getragen wird, ergeben sich Anreize zur interessenorientierten Ausweitung statlicher Ausgabentätigkeit.

Die Rent-Seeking-Vorhaben einzelner Gruppen besitzen dann hohe Erfolgsaussichten im politischen Prozeß, wenn sie gleichzeitig positive Externalitäten für andere gesellschaftliche Gruppen hervorrufen. Auf diese Weise lassen sich allokative staatliche Aktivitäten auf Distributionsmotive zurückführen, die mit Effizienzargumenten politisch opportun gerechtfertigt werden. Als zentrales Ergebnis ist festzuhalten, daß unter bestimmten Bedingungen aus dem Zusammenwirken von organisierten Gruppeninteressen und politischem Entscheidungsverhalten eine Zunahme des öffentlichen Sektors in demokratischen Staatswesen abgeleitet werden kann. 


\section{DAS DILEMMA DES POLITISCHEN INTERESSENWETTBEWERBS}

Die an Partikularinteressen orientierte Ausgestaltung der institutionellen Rahmenbedingungen individuellen Handelns ist das charakteristische Merkmal eines Vergünstigungsstaates. In diesem Kapitel wird der Frage nachgegangen, inwieweit ein kooperatives Verhalten der Interessengruppen im Hinblick auf die Vermeidung der beim Rent-Seeking induzierten Allokationsverluste erwartet werden kann. Zur Illustration des grundsätzlichen Unterschiedes zwischen dem Wettbewerb unter marktlichen und politischen Institutionen dient das spieltheoretische Konzept des "Gefangenendilemmas"1). Diese Form der Darstellung erlaubt es, die spezielle Interessenkonstellation beim Streben nach marktlichen bzw. nichtmarktlichen Renten aufzuzeigen und die daraus resultierenden gesellschaftlichen Konsequenzen zu veranschaulichen.

Das Rent-Seeking organisierter Gruppeninteressen hat mit dem Wandel der politischen Ordnung vom liberalen Rechtsschutzstaat zum Wohlfahrts-bzw. Interventionsstaat zunehmend an Bedeutung gewonnen ${ }^{2}$ ). Vor dem Hintergrund veränderter Legitimationsgrenzen für staatliches Handeln wird die gruppenspezifische Interessenstruktur unter diesen politischen Institutionen untersucht. In diesem Zusammenhang wird auch die Interdependenz zwischen distributiven Gruppeninteressen und Politikerverhalten deutlich, die einen wesentlichen Einfluß auf die Möglichkeiten zur Verminderung der Wohlfahrtsverluste des RentSeeking ausübt. Den Abschluß dieses Kapitels bildet eine Zusammenfassung der Resultate.

1) Zum spieltheoretischen Konzept des "Gefangenendilemmas" sei auf Luce/Raiffa (1967) verwiesen. Vgl. auch Tullock (1974b), S. $15 \mathrm{ff}$. bzw. Neumann (1975), S. $215 \mathrm{ff}$.

2) Vgl. Leipold (1988), S. 274. 


\section{Die Anreizstruktur beim Wettbewerb unter marktlichen Institutionen}

Die Analyse des Wettbewerbs im Marktsystem stellt die Grundlage für die Beurteilung der Konkurrenz im politischen Sektor dar. Die ökonomischen Konsequenzen des Interessenwettbewerbs finden ihren Niederschlag in den "payoffs" der in Tabelle 4 dargestellten Matrix. Dabei wird die Interessenkonstellation eines repräsentativen Mitgliedes der Gruppe $i(j)$ betrachtet, dessen ökonomische Position durch das jeweilige "Full Income" wiedergegeben wird. Diese Einkommensbeträge werden in monetären Größen gemessen und um monetäre Nutzenäquivalente korrigiert. Die Wirtschaftssubjekte können ihre Ressourcen jeweils zur Realisierung marktlicher Renten (Profit-Seeking) oder nichtmarktlicher Renten (Rent-Seeking) verwenden. Dabei symbolisiert die erste (zweite) Zahl in den Matrixfeldern das Einkommen, das ein Individuum der Gruppe $i(j)$ bei der entsprechenden Kombination der Handlungsalternativen erzielen kann ${ }^{1}$.

Tab. 4: Symmetrische Interessenstruktur beim Profit-Seeking

\begin{tabular}{|l|c|c|}
\hline$i$ & Profit-Seeking & Rent-Seeking \\
\hline $\begin{array}{l}\text { Profit- } \\
\text { Seeking }\end{array}$ & I & II \\
& $(10,10)$ & $(8,7)$ \\
Rent- & III & IV \\
Seeking & $(7,8)$ & $(6,6)$ \\
\hline
\end{tabular}

1) Vgl. dazu insbesondere die ausführliche Darstellung bei Ordeshook (1986), S. $203 \mathrm{ff}$. 
Aufgrund der Tatsache, "that full incomes can be added to measure aggregate income or aggregate outputn ${ }^{1)}$, wird das gesellschaftliche Ergebnis als Summe der Werte in den einzelnen Matrixfeldern ermittelt.

In der dargestellten Situation ist es für beide Individuen rational, die Ressourcen zur Erzielung marktlicher Renten und damit für produktive Zwecke einzusetzen. Beim Profit-Seeking wird das jeweils höhere Einkommen realisiert. Als Konsequenz der unabhängigen Verfolgung individueller Interessen ergibt sich die gleichgewichtige Lösung in Feld I der Matrix. Unter den gegebenen Bedingungen wird damit ein Pareto-optimales Ergebnis bewirkt, da jede andere Kombination der Handlungsalternativen einen geringeren Gesamtoutput impliziert. Das konstitutive Merkmal dieser Konstellation liegt in der Verbindung von privaten Interessen und marktlichem Wettbewerb ${ }^{2}$. "In an idealized model of market order, profit seeking as an activity produces consequences neither predicted nor understood by any single participant but 'good' when evaluated as a characteristic of the order itself" ${ }^{3)}$. Es stellt sich allerdings die Frage, inwieweit dieses Ergebnis Gültigkeit behält, wenn die Möglichkeit zur - verglichen mit der marktlichen Rentensuche ertragreicheren Ressourcenverwendung für distributive Zwecke besteht. Bei Existenz von begünstigenden staatlichen Institutionen werden bestimmte partikulare Verteilungsvorteile geschaffen, die eine Veränderung der "payoffs" der Matrix bewirken ${ }^{4}$ ).

1) Becker (1983), S. 374 .

2) Dieser Zusammenhang wird im allgemeinen mit der Metapher der "unsichtbaren Hand" des Marktmechanismus umschrieben.

3) Buchanan (1980a), S. 4.

4) Bei der Analyse von Zwei-Personen-Nichtnullsummenspielen wird die Problematik der Bildung von Koalitionen nicht berücksichtigt. 
Die in Tabelle 4 dargestellten Größenrelationen bleiben nur dann gewahrt, wenn eine vollständige Übertragung des Sondervorteils auf andere Gruppen und gleiche hohe Einflußnahmekosten für die beteiligten Parteien unterstellt werden. Darüber hinaus muß eine gleichmäßige Verteilung der Allokationsverluste vorliegen, damit die Gleichgewichtslösung in Feld I der Matrix resultiert, d.h. auf Rent-Seeking-Vorhaben verzichtet wird ${ }^{1}$.

In aller Regel sind diese Bedingungen nicht gegeben. Da marktliche Anpassungsreaktionen Zeit in Anspruch nehmen, ist von einer sofortigen Übertragung des Verteilungsvorteils nicht auszugehen, so daß zumindest in den Anfangsperioden ein Nettovorteil verbleibt. Hinzu kommt, daß sich die Einschätzung der eigenen "Full Incomes" eher an der Höhe der formalen Vergünstigung orientiert und weniger auf den nach AbschluB aller Anpassungsreaktionen resultierenden Verteilungsgewinn gerichtet ist. Aus diesen Gründen werden Anreize für Rent-SeekingProjekte induziert, deren reale Gewinne überschätzt werden. Die zunehmenden Rent-Seeking-Aktivitäten machen ihrerseits eine Strategie des Rent-Avoidance ertragreich, die sich auf die Verminderung der Verluste durch eine Vorteilsgewährung an andere Gruppen konzentriert. Zur Erweiterung der Analyse wird die Anreizstruktur betrachtet, die sich bei Existenz eines Rechtsschutzstaates bzw. eines Vergünstigungsstaates ergibt. Der Wettbewerb um Renten kann zu grundsätzlich anderen gesamtwirtschaftlichen Konsequenzen führen, wenn sich die institutionellen Bedingungen des individuellen Handelns ändern ${ }^{2}$.

1) Vgl. Folkers (1985), S. 287 .

2) Die unbeabsichtigten Wirkungen einer subjektiv rationalen Interessenverfolgung ergeben sich nicht "because individvals become different moral beings and modify their actions accordingly, but because institutional structure changes". Buchanan (1980a), S. 4. 


\section{Die Anreizstruktur beim Wettbewerb unter politischen} Institutionen

Die Beurteilung der Rolle des States beim Rent-Seeking muß in Verbindung mit dem existierenden Spielraum für öffentliche Aktivitäten erfolgen. Zur Sicherung der sozial wünschenswerten Ergebnisse sind Umfang und Inhalt der individuellen Eigentumsund Handlungsrechte zu definieren und zu schützen. Die Einhaltung und Durchsetzung des Rechtssystems macht eine Institution "Staat" erforderlich. Mit der Ausdehnung staatlicher Handlungsmöglichkeiten werden Anreize induziert, die Zwangsgewalt des Staates für Partikularinteressen zu nutzen. "With government as an instrument or vehicle ..., opportunities for gain, whether pecuniary or political or other advantage, accrue to those who can use government"1). Damit verlagert sich der Wettbewerb um Renten vom marktlichen in den politischen Sektor ${ }^{2}$.

\subsection{Rent-Seeking im Rechtsschutzstaat}

Aus der Rent-Seeking-Perspektive läßt sich eine ökonomische Begründung für die Existenz eines Staates entwickeln. Ausgangspunkt der Überlegungen sei der Zustand der Anarchie ${ }^{3}$ ), worunter die Bedingungen gesellschaftlichen Zusammenlebens ohne Staat verstanden werden. Die Individuen können ihre Ressourcen zur Güterproduktion (Profit-Seeking) oder zu Verteilungszwecken (Rent-Seeking) in Form von Diebstahl bzw. zur Sicherung ihres Eigentums verwenden.

1) Samuels (1971), S. 443 .

2) "As institutions have moved away from ordered markets toward the near chaos of direct political allocation, rent seeking has emerged as a significant social phenomenon." Buchanan (1980a), S. 4 .

3) Vgl. Nozick (1974) und Buchanan (1975). 
In Tabelle 5 wird die Interdependenz des individuellen Handelns für das kollektive Ergebnis deutlich, da die Reaktion des negativ betroffenen Individuums ausschlaggebend ist. Bei dieser Interessenlage besteht die Aussicht, bei produktiven Aktivitäten des anderen Individuums durch Rent-Seeking größere Vorteile als bei eigenem marktlichen Ressourceneinsatz realisieren zu können ${ }^{1)}$.

Tab. 5: Symmetrische Interessenstruktur beim Rent-Seeking im Rechtsschutzstaat

\begin{tabular}{|l|c|c|}
\hline$i$ & Profit-Seeking & Rent-Seeking \\
\hline $\begin{array}{l}\text { Profit- } \\
\text { Seeking }\end{array}$ & I & II \\
\hline Rent- & III 10$)$ & $(5,12)$ \\
Seeking & $(12,5)$ & IV \\
\hline
\end{tabular}

Andererseits besteht die Gefahr bei eigenem Profit-SeekingVerhalten durch die Umverteilungsaktivitäten des anderen Wirtschaftssubjektes noch schlechter gestellt zu werden als im Zustand beiderseitigen Rent-Seeking-Verhaltens (Feld II bzw. III im Vergleich zu Feld IV). In dieser Entscheidungssituation liegt für beide Individuen die vorteilhaftere separate Strategie in der Wahl der Alternative Rent-Seeking und zwar unabhängig davon, welche Annahmen über das Verhalten des Kontrahenten zugrunde gelegt werden. Das unintendierte Ergebnis ihrer individuell rationalen Entscheidung führt zu einer Situation, in der beide Wirtschaftssubjekte ein geringeres "Full Income" erzielen als bei gemeinsamer Verwendung der Ressourcen für produktive Zwecke.

1) Vgl. Buchanan (1980c), S. 362 . 
Das Dilemma besteht darin, daß jedes Individuum seine Entscheidung ohne Kenntnis der Strategienwahl der anderen Partei treffen muß, so daß für beide Akteure ein Anreiz zum RentSeeking besteht. Es ergibt sich ein gesamtwirtschaftliches Resultat, das keiner der Beteiligten beabsichtigt hat. Da beide Personen von den Allokationsverlusten gleichermaßen tangiert werden, handelt es sich um ein reines Allokationsproblem ${ }^{1}$.

Erkennen die Individuen allerdings, daß der für Eigentumsschutz und "Verteilungsfeldzüge" gebundene Ressourcenaufwand vermieden und für produktive Zwecke verfügbar gemacht werden kann, ist durch wechselseitige Anerkennung und Zusicherung von Eigentumsrechten die Realisierung des Pareto-superioren Resultates im Matrixfeld I möglich. Aus der Rent-Seeking-Perspektive kann damit die Bildung eines Staates ökonomisch aus dem Motiv der Kostenersparnis erklärt werden ${ }^{2}$. Solange die Funktion des Staates in der Definition der Grenzen legitimer Ausübung privater Handlungsrechte und deren Schutz besteht, wird er zur unverzichtbaren Voraussetzung für die Entfaltung produktiver Aktivitäten im marktlichen Wettbewerbsprozeß. Der Rechtsschutzstaat hat keine gesetzgeberische Funktion, sondern tritt den Individuen als Instanz zur Durchsetzung von Rechten gegenüber, auf die sie sich freiwillig geeinigt haben. Die Aufgaben des Staates lassen sich aber dahingehend erweitern, daß bestimmte öffentliche Güter - in der polaren Definition von Samuelson - bereitgestellt werden sollen. Damit ergibt sich der sogenannte "Leistungsstaat", der allerdings nicht das Recht hat, Transfers zwischen Individuen vorzunehmen ${ }^{3)}$.

1) Vgl. Buchanan (1980c), S. 362 .

2) Zur ökonomischen Begründung des Staates aus kontrakttheoretischer Sicht sei auf Nozick (1974) bzw. Buchanan (1975) verwiesen.

3) Zur Unterscheidung der Funktionen des Rechtsschutzstaates und des Leistungsstaates vgl. insbesondere Buchanan (1975), S. 68. Vgl. auch Brennan/Buchanan (1981), S. $112 \mathrm{f}$. 


\subsection{Rent-Seeking im Vergūnstigungsstaat}

Die Möglichkeit zur Veränderung der institutionellen Bedingungen des Marktprozesses mit Unterstützung des Staates durch die Realisierung politisch determinierter Ausnahme- und Sonderregelungen bedeutet "The Birth of a Transfer-Society"1). Die Ausdehnung staatlicher Aktivitäten interventionistischer Art induziert Anreize für Interessengruppen, durch politische Einflußnahme langfristig Verteilungsprivilegien zu sichern ${ }^{2}$. "Any portion of the society which can secure control of the state's machinery will employ the machinery to improve its own position"3). Für andere gesellschaftliche Gruppen implizieren die staatlich determinierten Institutionen zugunsten von Partikularinteressen entsprechende Belastungen. Die Interessenstruktur im Vergünstigungsstaat wird in Tabelle 6 dargestellt.

Tab. 6: Asymmetrische Interessenstruktur beim Rent-Seeking im Vergünstigungsstaat

\begin{tabular}{|l|c|c|}
\hline$i$ & Profit-Seeking & Rent-Seeking \\
\hline $\begin{array}{l}\text { Profit- } \\
\text { Seeking }\end{array}$ & $\begin{array}{c}\text { I } \\
(10,10)\end{array}$ & $\begin{array}{c}\text { II } \\
(6,13)\end{array}$ \\
\hline $\begin{array}{l}\text { Rent- } \\
\text { Seeking }\end{array}$ & III & IV \\
\hline
\end{tabular}

1) Anderson/Hill (1980). "Using the coercive power of the state discourage theft, but it opens another arena for transfer activity." Anderson/Hill (1980), S. 7.

2) Nach Ramb (1988), S. 247 erfüllen "staatliche Aktivitäten, die über die Unterstützung des Marktprozesses und die Sicherung seiner Ergebnisse ... hinaus gehen, ... immer den Tatbestand eines Diebstahls von Eigentum".

3) Stigler (1970), S. 1. 
Im Gegensatz zur Anreizstruktur im Rechtsschutzstaat liegt eine asymmetrische Struktur der Ertragsmatrix vor. Für das Mitglied der Interessengruppe $i$ ist es vorteilhaft, sich für die Alternative Rent-Seeking zu entscheiden, wenngleich der resultierende Verteilungsgewinn durch den Widerstand der Gruppe j gemindert wird ${ }^{1)}$. Wiederum ergibt sich die gleichgewichtige Lösung in Feld IV mit der Konsequenz von Allokationsverlusten durch die mit allen nichtneutralen Maßnahmen verbundene Mehrbelastung und den im Verteilungskonflikt verschwendeten Ressourcen ${ }^{2)}$. Der zentrale ökonomische Unterschied zur Konstellation im Rechtsschutzstaat liegt in der Vermengung von allokativen und distributiven Aspekten. Die asymmetrische Interessenstruktur verhindert ein effizientes Ergebnis, da Gruppe i ohne zusätzliche Kompensationszahlungen von $j$ nicht bereit sein wird, der Pareto-optimalen Lösung (Feld I) zuzustimmen. Es handelt sich damit um die Realisierung von Umverteilungsgewinnen unter "Inkaufnahme von Allokationsschäden"3). Das Rent-Seeking im Vergünstigungsstaat stellt sich somit als Negativ-Summenspiel ${ }^{4}$ ) dar, wobei die Asymmetrie der Interessenlage eine Einigung der Gruppen zum Verzicht auf Umverteilungsaktivitäten erschwert. Dieses Resultat ist von Bedeutung, wenn die "political prospects for institutional-legal changes that might be required to reduce the scope for rent seeking in modern society" ${ }^{5)}$ betrachtet werden.

1) Vgl. Buchanan (1980c), S. 362 .

2) Dabei wird davon ausgegangen, daß sich die Allokationsverluste gleichmäßig verteilen und beide Gruppen in gleichem Umfang Ressourcen zur Produktion politischen Drucks aufgewendet haben.

3) Folkers (1985), S. $296 \mathrm{ff}$.

4) "The transfer is negativ sum since nothing is produced and resources are expended in the process." Anderson/Hill $(1980)$, S. 6 .

5) Buchanan (1980c), S. 359. 
Da die Verteilungsgewinne und -verluste als folge von politischen Entscheidungen resultieren, werden sich die Verlierer weigern, die notwendige Kompensation zu leisten ${ }^{1)}$. Durch eine Zahlung würden sie nämlich den Anspruch der anderen Gruppe anerkennen, den diese aus ihrer Sicht unberechtigterweise bzw. durch Glück erreicht hat. Da die belastete Gruppe ebenfalls Ressourcen in den politischen Prozeß investiert hat, besteht ihre Hoffnung in der Revision der als ungerecht bzw. unmoralisch empfundenen Begünstigung der anderen Gruppe.

Diese Einstellung wird noch verstärkt, wenn die belasteten Wirtschaftssubjekte davon ausgehen, daß sie im Verteilungskampf a priori benachteiligt waren ${ }^{2}$. Wenngleich ex ante eine symmetrische Erfolgswahrscheinlichkeit zur Realisierung des Distributionsvorteils vorgelegen hat, resultiert nach der ersten Runde ein Vorteil zugunsten der Gruppe i, so daß sich ex post die asymmetrische Struktur (Tabelle 6) ergibt ${ }^{3}$ ). Während die Begünstigten den Verteilungsvorteil als gesichertes Recht und legitimen Besitzstand betrachten, werden die Belasteten die für eine Effizienzsteigerung notwendigen Kompensationszahlungen nach einem intensiven Verteilungskonflikt noch weniger zu leisten bereit sein, wenn sie der Ansicht sind, "that the payoff structure was nonsymmetrical even in the ex ante sensen ${ }^{4}$ ).

1) Vgl. Buchanan $(1980 \mathrm{c}), \mathrm{S}, 364$.

2) D.h. in der Ausgangssituation kann sowohl Gruppe i als auch $j$ den Vorteil in Matrixfeld IV erwarten, so daß die Konstellation $(11,7)$ bzw. $(7,11)$ mit gleicher Wahrscheinlichkeit eintritt. Vgl. Buchanan (1980c), S. $364 \mathrm{f}$. bzw. Rowley $(1988$ b), S. 459.

3) Wird die Existenz dieser Asymmetrie auch für weitere Konfliktrunden unterstellt, ist weiterhin die nichtkooperative Lösung zu erwarten. Die Problematik der Entstehung und Stabilität kooperativer Verhaltensweisen bildet den Gegenstand der Untersuchung von Axelrod (1984).

4) Buchanan $(1980$ c), S. 364 . 
Darüber hinaus ist zu berücksichtigen, daß die meisten Individuen als Mitglieder verschiedener Gruppen (Produzent, Steuerzahler, Konsument etc.) gleichzeitig in mehreren Konflikten auf verschiedenen Seiten beteiligt sind und sich jeweils anderen Opponenten gegenübersehen. Unter diesen Bedingungen werden sich aus dem Prozeß heraus keine Institutionen entwickeln, die zur Vermeidung der Allokationsverluste beitragen. Insbesondere die mangelnde Information der Beteiligten bezüglich der konkreten Opposition, der Höhe der tatsächlichen Verteilungsgewinne bzw. -verluste, die Identifikation der effektiv Begünstigten und Belasteten führen dazu, daß die nichtkooperative Lösung mit der Folge der sozialen Verluste realisiert wird ${ }^{1}$.

Ebenso werden die Wirtschaftssubjekte, die nicht unmittelbar am Distributionskonflikt beteiligt sind, durch die RentSeeking-Aktivitäten anderer Interessengruppen tangiert. Da sich einerseits bestimmte Vorteile ergeben, denen andererseits Nachteile gegenüberstehen, sind eher unsystematische Nettoeffekte zu erwarten. Obgleich sich die Verteilungswirkungen für diese Individuen ausgleichen können, werden sie dennoch von den Allokationsverlusten betroffen ${ }^{2}$. Die Wirtschaftssubjekte erleiden somit Nutzeneinbußen durch Mehrbelastungen und durch die Ressourcenverschwendung im Verteilungskampf, während die resultierenden Verteilungsgewinne letztlich unsicher und relativ gering sein werden ${ }^{3)}$.

1) Außerdem verliert bei ständig wechselnden Konkurrenten die Gefahr von Vergeltungsmaßnahmen an Bedeutung, da sich keine Erfahrungen mit bestimmten gegnerischen Interessengruppen herausbilden können. Zur Strategie des "Tit for Tat" sei auf Weck-Hannemann (1988), S. 185 ff. verwiesen.

2) Vgl. Folkers (1983a), S. 198.

3) Vgl. dazu Tietzel (1988b), S. 98. 
Aufgrund der Tatsache, daß viele Gruppen den Negativsummencharakter des Distributionskonflikts nicht erkennen und einen Umverteilungskonflikt in Form eines Nullsummenspiels unterstellen, wird ein kooperatives Verhalten kaum in Betracht gezogen ${ }^{1)}$. Aus diesem Grund ist eine Verstärkung der RentSeeking-Bemühungen der einzelnen Interessengruppen zu erwarten, die eine Zunahme der damit verbundenen Wohlfahrtsverluste impliziert.

Dieser Prozeß wird durch die Dynamik der Übertragung von Verteilungsvorteilen gefördert, die eine Akzeleration der RentSeeking-Vorhaben bewirken ${ }^{2}$ ). Dieser Mechanismus stellt die Grundlage für die permanente Forderung nach weiteren Vergünstigungen dar, wenn die formalen Vorteile durch marktliche Anpassungsreaktionen vermindert werden. Gleichzeitig werden alle Vorschläge zum Abbau derartiger Vergünstigungen auf den erbitterten Widerstand der betroffenen Gruppen stoßen, da auf diese Weise reale Verluste hervorgerufen werden. Dieses Verfahren ist auch für die Gruppenfunktionäre geeignet, da sie ihre individuellen Ziele nur mit Unterstützung ihrer Mitglieder und durch die Bestätigung in ihrem Amt bei erfolgreich abgeschlossenen Projekten verwirklichen können.

Die rationale Informationsasymmetrie bei den einzelnen Individuen führt dazu, daß zwar die eigenen Vorteile gesehen, die Nachteile durch Sonderregelungen für andere Gruppen allerdings kaum beachtet werden. Daher wird die Opposition gering ausfallen und sich eher auf die Gewinnung neuer Verteilungsvorteile konzentrieren.

1) Vgl. Folkers (1983a), S. 198.

2) Vgl. Folkers (1985), S. 297. 
Für die politischen Entscheidungsträger bestehen Anreize, den Gruppenforderungen nachzukommen. Begünstigende Institutionen werden verabschiedet, wenn ein Zuwachs an politischer Unterstützung in Form von Wählerstimmen bzw. finanziellen oder informationsmäßigen Ressourcen zu erwarten ist. In gleicher Weise entspricht das Rent-Seeking privater Interessengruppen den Zielsetzungen der Bürokraten. Die Zunahme staatlicher Regelungen und Maßnahmen sichert nicht nur den Bestand an Ämtern, sondern erweitert deren Zuständigkeitsbereich ${ }^{1}$.

Mit der zunehmenden Erodierung marktlicher Institutionen zugunsten von Sonderinteressen nimmt die Entwicklungsdynamik des marktwirtschaftlichen Allokationssystems ab. Die partikular begünstigenden Maßnahmen induzieren Anpassungsreaktionen, die wiederum Folgeinterventionen ${ }^{2}$ ) bedingen und die Funktionsfähigkeit des Marktprozesses beeinträchtigen ${ }^{3}$. Dieser Prozeß fördert das Wachstum des staatlichen Sektors zu Lasten des marktlichen Sektors einer ökonomie. Alle Gruppen werden in beträchtlichem Ausmaß Ressourcen zur Durchsetzung ihrer gruppenspezifischen Interessen im Kampf um Verteilungsprivilegien einsetzen, ohne daß die resultierenden, polititisch determinierten Regelungen im Einklang mit gesellschaftlich erwünschten Ergebnissen stehen. Damit ergibt sich die Notwendigkeit zur Entwicklung von Reformvorschlägen, die zur überwindung des verteilungspolitischen Dilemmas in demokratischen Staatswesen beitragen.

1) Vgl. Leipold (1988), S. 265.

2) Wird beispielsweise die Stahlindustrie geschützt, verlangen auch Lieferanten (Kohlebergbau) und Abnehmer (Metallindustrie) staatliche Garantien. Die sinkende Produktivität und Wettbewerbsfähigkeit einer Volkswirtschaft ist weniger auf den einzelnen staatlichen Eingriff als auf die dadurch induzierte Interventionsspirale zurückzuführen.

3) Vgl. Streit $(1988 \mathrm{~b})$, S. $49 \mathrm{f}$. 


\section{Zusammenfassung}

Im Rahmen einfacher spieltheoretischer Überlegungen wird gezeigt, daß aufgrund der speziellen Interessenkonstellation beim Distributionskonflikt ein mögliches kooperatives Verhalten zur Minderung der Ressourcenverschwendung beim RentSeeking von Interessengruppen nicht zu erwarten ist. Mit der Zunahme distributiv motivierter Institutionen werden Verteilungsfragen in steigendem Maße durch politische Prozesse und entsprechend weniger durch Marktprozesse gelöst. Damit werden Anreize geschaffen, produktive Aktivitäten im Marktsektor durch distributive Vorhaben über den politischen Sektor zu substituieren. Der Wettbewerb um politische Renten kann den Wettbewerb um marktliche Renten verdrängen.

Die fortschreitende Verlagerung der Verteilungsentscheidungen in den politischen Bereich entspricht den Interessen der Politiker, die aufgrund ihrer individuellen Zielsetzungen keinen Anreiz haben, kollektive Interessen zur Geltung zu bringen. Da sich die Gewährung von Sonderregelungen gegenüber der Aufrechterhaltung allgemeingültiger Institutionen als politisch vorteilhaft erweist, werden die Politiker den Gruppenforderungen im Rahmen ihrer legalen Kompetenzen nachkommen.

Die Unvollkommenheiten des politischen Wettbewerbs und die Verteilungseffekte marktlicher Anpassungsreaktionen bewirken eine Akzeleration von Rent-Seeking-Vorhaben von Interessengruppen mit der Folge, daß weitere Ressourcen in unproduktive Verteilungskonflikte investiert werden. Letztlich werden zahlreiche Gruppen bestimmte Vorteile realisieren, ohne sich bewußt zu sein, daß sie im Gegenzug durch die politischen Vergünstigungen anderer gesellschaftlicher Gruppen belastet werden. Dabei ist weniger die einzelne staatliche Maßnahme von Bedeutung als vielmehr die Kumulation der vielen Eingriffe, die eine "Sklerose" des Gesamtsystems bewirkt und zu einer ineffizienten Allokation der volkswirtschaftlichen Ressourcen beiträgt. 


\section{ZUR REFORM DER "RENT-SEEKING-SOCIETY"}

Vor dem Hintergrund der Interessenkonstellation beim RentSeeking erweisen sich staatlich determinierte Institutionen sowohl für die Interessengruppen als auch für die politischen Entscheidungsträger als geeignete Instrumente zur Realisierung ihrer jeweiligen Zielsetzungen. Die positive Analyse des Zustandekommens staatlicher Regelungen in demokratischen Staatswesen zeigt, daß die ständige Ausdehnung des öffentlichen Sektors im Zuge des Rent-Seeking-Verhaltens von Interessengruppen und die damit verbundenen Allokationsverluste das Resultat bestehender Institutionen sind. Aufgrund der speziellen Anreizstruktur beim Rent-Seeking werden an Interessengruppen und Politiker gerichtete Appelle zu einem Verhalten im Sinne des "Gemeinwohls" wirkungslos bleiben, solange die grundlegenden Restriktionen des politischen Entscheidungsverfahrens nicht entsprechend umgestaltet werden.

Aus diesen überlegungen ergeben sich Ansatzpunkte für Reformvorschläge, die auf eine Veränderung der institutionellen Bedingungen politischen Handelns abzielen. Im Mittelpunkt steht die Nevorientierung der Ordnungspolitik, die sich auf die Schaffung funktionsfähiger Restriktionen für staatliche Aktivitäten konzentriert ${ }^{1)}$. Aufgrund der Interdependenz zwischen politischem Entscheidungsverhalten und den Forderungen von organisierten Interessengruppen muß sich eine wirksame Ordnungspolitik auf den marktlichen und den öffentlichen Sektor richten, um gesellschaftlich wünschenswerte Resultate zu ermöglichen ${ }^{2}$.

1) Vgl. Folkers $(1984 a)$, S. 502.

2) Vgl. grundlegend Brennan/Buchanan (1985) bzw. Kirchgässner (1988), S. $53 \mathrm{ff}$. und Buchanan/Vanberg (1988), S. $101 \mathrm{ff}$. 


\section{Zur Neuorientierung der Ordnungspolitik}

Die Zunahme staatlicher Interventionen ist das Ergebnis erfolgreicher Einflußnahmeaktivitäten von seiten einzelner Interessengruppen ${ }^{1)}$. Eine Möglichkeit zur Verminderung des Rent-Seeking besteht in einer stärkeren Reglementierung von Interessengruppen. "Die Politik des Staates sollte darauf gerichtet sein, wirtschaftliche Machtgruppen aufzulösen oder ihre Funktionen zu begrenzen"2). Die im Grundgesetz verankerte Koalitionsfreiheit garantiert das Recht. Interessengruppen zum Zwecke der Förderung von gemeinsamen Gruppeninteressen zu bilden. Darüber hinaus ist die Existenz von Interessengruppen aus ökonomischer Perspektive durchaus positiv zu bewerten, da sie eine wichtige Informationsfunktion im politischen Prozeß wahrnehmen und zur Verminderung von Informationskosten beitragen. Aus diesem Grund richten sich Reformvorschläge für die Gestaltung des Interessenwettbewerbs in erster Linie darauf, die Einflußnahme von organisierten Interessengruppen auf die Entscheidungen staatlicher Instanzen transparent $z u$ machen ${ }^{3}$ ) bzw. verbesserte Bedingungen für den Wettbewerb zwischen Interessengruppen zu schaffen ${ }^{4}$.

1) "Die Wirtschaftsverbände bemächtigen sich also des staatlichen Apparates und versuchen, ihn im Dienste ihrer gruppenegoistischen Interessen einzusetzen." Werner (1957), S. 208.

2) Eucken (1975), S. 334 .

3) Vgl. Koford/Colander (1984), S. 207. In diesem Zusammenhang sind $z$.B. öffentliche Anhörungen von Verbänden bzw. die Publikation ihrer Eingaben zu Gesetzesvorhaben vorgesehen. Die wirkungsvollsten Einflußnahmeaktivitäten finden allerdings nicht öffentlich statt oder kommen dadurch zustande, daß politische Entscheidungsträger gleichzeitig Vertreter organisierter Gruppen sind.

4) Vgl. Watrin (1973), S. 81 f., der in der Privilegierung einzelner Gruppen (z.B. Gewerkschaften, Arbeitgeberverbände) im Hinblick auf ihre verfassungsrechtliche Stellung einen Verstoß gegen das Prinzip der Gleichbehandlung sieht. 
Allerdings erscheinen solche Versuche, das gruppenspezifisch rationale Rent-Seeking durch Reglementierungen auf seiten der "Nachfrage" nach statlichen Privilegien zu vermindern, aufgrund der speziellen Interessenkonstellation bei Verteilungskonflikten wenig erfolgversprechend. Interessengruppen werden zur Teilnahme am Distributionswettbewerb gezwungen sein, weil die erfolgreiche Einflußnahme anderer Gruppen Nachteile hervorruft.

Eine Reform muß vielmehr an den Ursachen für die Rent-SeekingAktivitäten ansetzen, die in der politischen Interventionsbereitschaft ${ }^{1}$ ) und der institutionell nahezu unbeschränkten Kompetenz des Parlaments liegen ${ }^{2}$. Die Möglichkeit der Politiker, den Gruppeninteressen nachzukommen bzw. ihrerseits den Interessengruppen "Angebote" $z u$ machen, stellt eine wesentliche Triebfeder für die ständige Zunahme punktueller Eingriffe zugunsten von Partikularinteressen dar. Die Tatsache, daß die gegebenen Restriktionen für den politischen Sektor unzureichend ausgestaltet sind, kennzeichnet den "fatalen Defekt der vorherrschenden Form der Demokratien ${ }^{3}$ ).

1) Die Bereitschaft der Politiker zu punktuellen Interventionen eröffnet neve Rent-Seeking-Möglichkeiten und "provides the economic foundations to most special-interest-groups". Streit (1987), S. 11.

2) Vgl. Streit (1983), S. 177.

3) Hayek (1979), S. 3. Im Mittelpunkt des Reformkonzeptes von Hayek steht die Einrichtung eines parlamentarischen Zweikammersystems, das durch eine klare Trennung der jeweiligen Zuständigkeiten und durch unterschiedliche Wahlverfahren für die Mitglieder gekennzeichnet ist. Zur Diskussion dieses Reformvorschlags sei auf Rupp (1979), S. $95 \mathrm{ff}$. bzw. Nienhaus (1982) verwiesen. 
Die ökonomisch negativen Konsequenzen des Rent-Seeking werden durch das Zusammenwirken von Interessengruppen und politischen Entscheidungsträgern hervorgerufen und hängen wesentlich von der Ausgestaltung der "Spielregeln" ab, denen diese Akteure bei ihrem Handeln unterworfen sind ${ }^{1)}$. Der institutionelle Rahmen des politischen Entscheidungsverfahrens induziert Verhaltensanreize, die eine Divergenz $z$ wischen individueller und kollektiver Rationalität bewirken ${ }^{2)}$. Die positive Analyse des Distributionswettbewerbs in demokratischen Systemen führt zu dem Ergebnis, daß eine Aushöhlung des marktlichen Ordnungsrahmens durch permanente politische Interventionen nur dann zu verhindern ist, wenn eine Ordnungspolitik für den öffentlichen Sektor formuliert wird. Der Grundgedanke einer Ordnungspolitik für beide Bereiche des ökonomischen Handelns wird in übersicht 3 veranschaulicht.

Gegenstand der Ordnungspolitik in demokratischen Systemen ist in herkömmlicher Sicht die Gestaltung der marktlichen Institutionen, innerhalb derer individuelle Initiativen entfaltet werden. Im Rahmen einer Marktwirtschaft sind die privaten Eigentumsrechte zu sichern und Institutionen zum Schutz des marktlichen Wettbewerbs zu gewährleisten. Die institutionellen Rahmenbedingungen marktwirtschaftlicher Prozesse finden ihren Niederschlag in einer "Wirtschaftsverfassung". Die Aufgabe der Wirtschaftsordnungspolitik besteht darin, "ein auf Dauer angelegtes, für alle verbindliches system rechtlich normierter Verhaltensregeln für die Beteiligung am Wirtschaftsleben zu schaffen, anzuwenden und durchzusetzen" ${ }^{3)}$.

1) Vgl. Vanberg (1986), S. $84 \mathrm{ff}$.

2) "Die Neue Politische ökonomie hat die Augen dafür geöffnet, daß zwischen dem ökonomisch Rationalen und dem politisch Rationalen eine - bisweilen sehr erhebliche - Diskrepanz bestehen kann." v. Arnim (1987), S. 26.

3) Cassel (1988), S. 316. 
Übersicht 3: Zur Neuorientierung der Ordnungspolitik

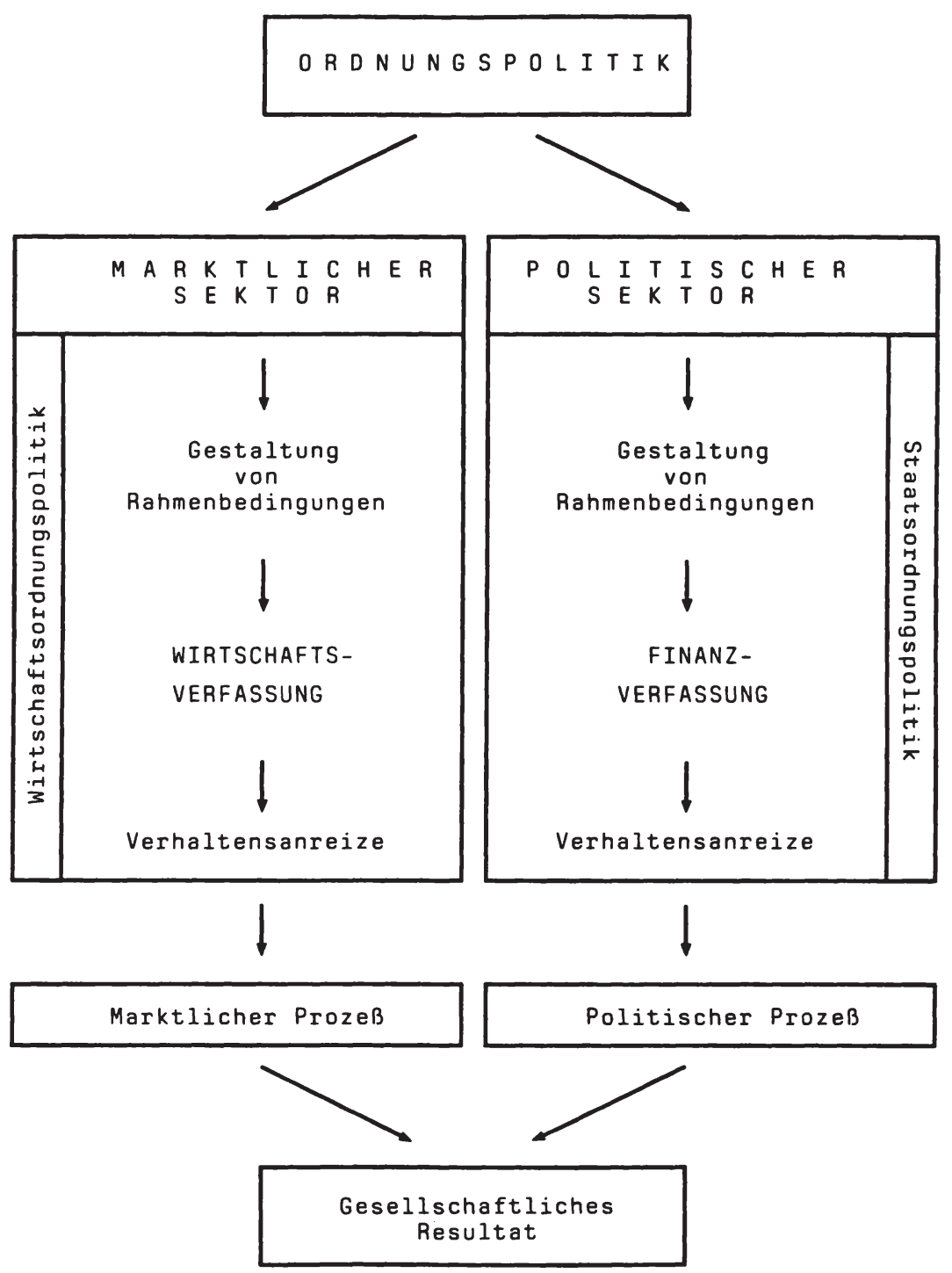


Entsprechend dieser Auffassung richtet sich die ordnungspolitische Gestaltung marktlicher Beziehungen auf die Schaffung geeigneter Anreize für die auf freiwilliger Basis stattfindenden Tauschprozesse und nicht auf das resultierende Verteilungsergebnis. Im Rahmen der traditionellen Analyse wird allerdings die Frage vernachlässigt, ob eine wirksame Wirtschaftsordnungspolitik überhaupt im politischen Prozeß realisiert werden $\operatorname{kann}^{1)}$.

Die von "Verteilungskoalitionen" bestimmte Wirtschaftspolitik modifiziert durch ständige Interventionen die Struktur der privaten Eigentums- und Handlungsrechte und erzeugt Anreize zur Durchsetzung von gruppenspezifisch begünstigenden Institutionen. Die Aussicht, Verteilungsvorteile auf politischem Wege zu sichern, fördert die Bildung organisierter Interessenvertretungen und induziert Rent-Seeking-Aktivitäten. Die ökonomische Analyse politischen Verhaltens verdeutlicht, daß die Politiker unter den gegebenen institutionellen Bedingungen des Entscheidungsverfahrens eine an Gruppeninteressen orientierte Politik interventionistischer Art einer längerfristig geltenden, die Verfahrensregeln gestaltenden Ordnungspolitik vorziehen ${ }^{2}$. Die allgemeinen politischen Institutionen der Demokratie ( $z . B$. Wahlregeln) weisen bestimmte Unvollkommenheiten auf, die eine gewisse Eigendynamik staatlichen Handelns beim Rent-Seeking mit der Folge sozialer Verluste implizieren.

1) Vgl. dazu Eucken (1959), S. 156 ff. Dort wird die Analyse politischer Prozesse ausgeklammert. Zu den bekannten "Prinzipien der Wirtschaftspolitik" sei auf Eucken (1975), S. $254 \mathrm{ff}$. verwiesen. Zur Analyse der deutschen Tradition ordnungstheoretischen Denkens aus der Perspektive der modernen konstitutionellen ökonomie vgl. Vanberg (1988), S. $18 \mathrm{ff}$.

2) Auf diese Weise wird die "Konstanz der Wirtschaftspolitik", die nach Eucken ein zentrales Erfordernis für eine funktionierende Marktwirtschaft darstellt, durch den politischen Prozeß konterkariert. Vgl. Eucken (1975), S. $285 \mathrm{ff}$. 
Es stellt sich damit die Frage, wie das politische Entscheidungsverfahren bei gegebenen politischen Institutionen verbessert werden kann, damit gesellschaftlich wünschenswerte Resultate ermöglicht werden. Zu diesem Zweck ist eine Ordnungspolitik für den öffentlichen Sektor zu entwerfen, die in Gestalt einer Finanzverfassung konkretisiert wird. Die Funktion der Finanzverfassung besteht darin, durch fiskalische Restriktionen den unerwünschten Tendenzen des politischen Verfahrens entgegenzuwirken und Anreize für Verhaltensweisen zu geben, die im Einklang mit kollektiven Interessen stehen. Die Entwicklung einer Ordnungspolitik für den öffentlichen Sektor beruht auf der überlegung, daß durch eine geeignet gestaltete Finanzverfassung die gegebenen Unvollkommenheiten der demokratischen Institutionen kompensiert werden können. Aus dieser Perspektive ergibt sich eine Analogie zur Theorie des Second-Best ${ }^{1}$. die in bestimmten Bereichen eine gezielte Abweichung von an sich optimalen Verfahren nahelegt, um bestehenden Verzerrungen in anderen Sektoren entgegenzuwirken und damit eine Verbesserung des Gesamtresultates zu erreichen.

Die Aufgabe der "Staatsordnungspolitik"2) besteht darin, Regeln festzulegen, nach denen das politische Entscheidungsverfahren durchgeführt werden soll. Damit wird nicht auf das konkrete Ergebnis abgestellt, sondern auf die Bedingungen, unter denen der Entscheidungsprozeß abläuft.

1) Vgl. grundlegend Lipsey/Lancaster (1956/57), S. $11 \mathrm{ff}$. bzw. im gegebenen Zusammenhang Folkers (1984a), S. 505.

2) Die Staatsordnungspolitik wird in demokratischen Staatswesen auch als "Demokratiepolitik" bezeichnet. Vgl. Tietzel (1988b), S. 102. Vgl. auch Cassel (1988), S. 319. 
Der für den Bereich der Wirtschaftsordnung allgemein akzeptierte Gedanke einer Ordnungspolitik ist auch auf den Bereich der politischen Willensbildung zu übertragen, um die Funktionsfähigkeit des Marktsystems nicht durch den politischen Interventionismus zu beeinträchtigen ${ }^{1)}$. Nur das Zusammenwirken einer Ordnungspolitik für marktliches und politisches Handeln kann eine Eindämmung der allokativen Verluste des Rent-Seeking bewirken und die Realisierungschancen für gesellschaftlich wünschenswerte Ergebnisse erhöhen.

Diese überlegungen schließen nicht aus, daß demokratisch herbeigeführte Ergänzungen bzw. Korrekturen der unter den marktlichen Institutionen erzeugten Verteilung stattfinden können. Im Rahmen der Konzeption einer "Sozialen Marktwirtschaft"2) wird eine Verbindung zwischen dem Prinzip der Freiheit am Markt und dem Prinzip des sozialen Ausgleichs ${ }^{3}$ ) angestrebt. Vom Standpunkt sozialer Zielsetzungen ist der gewünschte Verteilungseffekt allerdings eher zu erreichen, wenn eine Unterstützung von seiten des Staates nach Einkommensgesichtspunkten und nicht nach branchenspezifischen, sektoralen oder anderen gruppenspezifischen Merkmalen erfolgt. Auf diese Weise lassen sich sozialpolitische Aspekte berücksichtigen, ohne durch partikular begünstigende Eingriffe den Marktmechanismus außer Kraft zu setzen und unsystematische Verteilungswirkungen im Wege des Rent-Seeking zu erzeugen.

1) Die unerwünschten Wirkungen, die durch den politischen Interventionismus hervorgerufen werden, sind die Konsequenz einer "Anmaßung von Wissen" von seiten der Politiker. Vgl. Hayek $(1975)$, S. 12 .

2) Vgl. dazu den Überblick von Böbel (1988), S. 135 ff.

3) Vgl. Watrin (1980), S. $476 \mathrm{ff}$. 


\section{Rent-Seeking aus konstitutioneller Perspektive}

Zur Entwicklung einer Ordnungspolitik für den öffentlichen Sektor besteht die Notwendigkeit, zwischen der Ebene der konstitutionellen Entscheidungen - im Sinne von Entscheidungen über Regeln von gewisser Dauer - und der nachgelagerten Ebene der laufenden Entscheidungen im Rahmen gegebener Regeln zu unterscheiden $^{1)}$. Staatliche Institutionen sind als Rahmenbedingungen für wirtschaftliche und politische Entscheidungen zu betrachten, die langfristig Gültigkeit besitzen und die Voraussetzung für effiziente nachkonstitutionelle Entscheidungen darstellen. Auf der konstitutionellen Ebene wird entschieden, welche Aufgaben der Staat wahrnehmen soll und welche Entscheidungsregeln bei bestimmten fragen anzuwenden sind.

Ausgangspunkt des institutionellen Entscheidungsproblems ist die Annahme, daß ein Individuum keine Informationen über seine konkrete Position in späteren Perioden besitzt. Hinter diesem "Schleier der Unwissenheit"2) wird sich das Individuum für bestimmte Regeln entscheiden, die sich nicht auf "Vorteilhaftigkeit im Einzelfall sondern auf präferierte Verfahren" ${ }^{3}$ ) beziehen, unabhängig von der konkreten individuellen Situation. Auf diese Weise wird eine Übereinstimmung über statliche Institutionen ermöglicht. Das normative Kriterium für eine Finanzverfassung ist ihre einstimmige Verabschiedung. Es liegt in der Logik des konstitutionellen Ansatzes, daß die Grundlage einer Legitimation staatlichen Handelns allein in der Zustimmung der beteiligten Individuen zu finden ist. Entsprechend dieser Sichtweise sind auch institutionelle Reformvorschläge diesem Kriterium zu unterwerfen.
1) Vgl. Buchanan (1975).
2) Vgl. Rawls (1971), S. $136 \mathrm{ff}$.
3) Folkers (1986a), S. 9 f. 
In Analogie zu marktlichen Transaktionen, die im Wege des freiwilligen Austausches von Rechten effiziente Ergebnisse bewirken, sind kollektive Entscheidungen, die auf der Zustimmung aller beteiligten Individuen beruhen, ebenfalls effizient ${ }^{1}$. Einstimmig beschlossene fiskalische Regeln genügen darüber hinaus auch den "Kriterien der Fairneß". Da jedes Individuum sein Veto einlegen kann, falls es sich durch bestimmte Entscheidungen beeinträchtigt fühlt, können die verabschiedeten Regeln als gerecht bezeichnet werden. Weil die Individuen keine Informationen darüber besitzen, ob sie bei Anwendung bestimmter Institutionen in späteren Perioden zu den Gewinnern oder Verlierern gehören, werden sie bei risikoaverser Einstellung ${ }^{2)}$ beide Möglichkeiten berücksichtigen und sich für entsprechende Regelungen auf der Ebene des gesellschaftlichen Grundkonsenses entscheiden ${ }^{3}$ ).

Welche Institutionen das Individuum auf konstitutioneller Ebene wählen wird, hängt von den Erwartungen hinsichtlich der Eigenschaften der ökonomischen und politischen Prozesse auf der nachkonstitutionellen Ebene ab. Die Analyse des staatlichen Handelns im Vergünstigungsstaat führt zu dem Ergebnis, daß demokratische Mehrheitsentscheidungen keine hinreichenden Vorkehrungen gegen unerwünschte finanzwirtschaftliche Maßnahmen darstellen und das Verfahren der Vorteilsgewinnung durch Rent-Seeking begünstigen. Erkennen die Individuen die negativen ökonomischen Konsequenzen der distributiven Negativsummenspiele, werden sie sich für Regelungen zur Eindämmung der sozialen Schäden des Rent-Seeking entscheiden.

1) Vgl. auch Vanberg (1981), S. $33 \mathrm{f}$.

2) Vgl. dazu kritisch West/Corke (1980), S. 395 ff.

3) Da der Verteilungswettbewerb in einer gegebenen Periode stets Nullsummencharakter hat, ist ein Konsens auf der Ebene der laufenden Entscheidungen nicht zu erzielen. 
Von seiner Position hinter dem Schleier der Unwissenheit wird ein rational handelndes Individuum Regeln präferieren, die einer Verschwendung von Ressourcen durch Rent-Seeking vorbeugen und die Möglichkeiten zur Gewährung von Sonderprivilegien im interessenorientierten Vergünstigungsstaat begrenzen ${ }^{1)}$. Wenngleich das Konzept der konstitutionellen Entscheidung im "Urzustand" lediglich als theoretisches Konstrukt zu verstehen ist, lassen sich daraus Anhaltspunkte für Reformvorschläge gewinnen. Auf welche Einschränkungen der "Macht zur Umverteilung" ${ }^{2)}$ werden sich Individuen einigen, um die Allokationsverluste des Rent-Seeking zu vermindern?

\section{Ansatzpunkte für institutionelle Reformen}

Die im Wege des Rent-Seeking erzeugten politischen Entscheidungen werden durch unzureichende institutionelle Beschränkungen des Handlungsspielraums der Politiker ermöglicht. Neben fiskalischen Restriktionen sind allerdings ergänzende Regelungen notwendig, um das Streben nach außerbudgetären Vergünstigungen bzw. vorteilhaften Regulierungen einzuschränken ${ }^{3}$ ) und Anreize für einen verstärkten Ressourceneinsatz im Marktsektor zu induzieren. Die Entwicklung von institutionellen Lösungskonzepten muß sich auf die analysierten Interessen und Verhaltensmuster der am Rent-Seeking-Prozeß beteiligten Akteure beziehen. Nach der Darstellung einzelner Reformvorschläge wird auf Realisierungschancen und Implementationsprobleme eingegangen.

1) Vgl. Brennan/Buchanan (1980), S. 31.

2) Vgl. Brennan/Buchanan (1981), S. $103 \mathrm{ff}$.

3) "In attempting to control existing forms of rent-seeking new rent-seeking opportunities will be created." Crew (1987), S. 161 . 


\subsection{Begrenzung politischer Handlungsspielräume}

Grundsätzlich lassen sich verfahrensmäßige und ergebnisbezogene Beschränkungen unterscheiden ${ }^{1)}$. Im Gegensatz zu den ergebnisbezogenen Restriktionen, die starr und teilweise willkürlich bestimmte feste Ergebnisse in Form von Maximalwerten vorgeben, besitzen prozedurale Regeln den Vorteil, daß sie flexible Anpassungen des staatlichen Handelns an geänderte Konstellationen ermöglichen. Allerdings sind quantitative Beschränkungen häufig in Kombination mit verfahrensmäßigen Regeln geeignet, als übergangsregelungen bzw. in Form von Konsolidierungsverfahren bestimmte finanzwirtschaftliche Fehlentwicklungen zu korrigieren ${ }^{2}$ ).

Da die Vermengung staatlicher Maßnahmen im Allokations- und Distributionsbereich Anreize zu Rent-Seeking-Aktivitäten induziert, ist eine strikte Trennung beider Funktionen ${ }^{3)}$ des öffentlichen Sektors notwendig. Zur Erhöhung der Transparenz von budgetären Entscheidungsprozessen sollte eine Integration von Steuer- und Ausgabenentscheidungen ${ }^{4)}$ erfolgen. Bei gleichzeitiger Betrachtung von spezifischen Ausgaben und Besteuerung ist eine Verschleierung der Kosten bestimmter Ausgabenkategorien nicht mehr möglich.

1) Zur Klassifikation und Systematisierung von fiskalischen Restriktionen sei auf Folkers (1983b), S. $27 \mathrm{ff}$. verwiesen.

2) Vgl. dazu das Verfahren zum Abbau des Budgetdefizits in den USA, das auf einem Kürzungsautomatismus mit bestimmten quantitativen Vorgaben für das maximal zulässige Defizit in den einzelnen Haushaltsjahren beruht. Vgl. dazu Folkers $(1986 b)$, S. 387 .

3) Vgl. dazu insbesondere Musgrave (1974), S. 3 ff.

4) Vgl. zu diesem Vorschlag bereits Wicksell (1896), S. 115. 
Zur Verminderung der zahlreichen partikularen Vergünstigungen im Steuer- und Ausgabenbereich ist eine verstärkte Anwendung des Äquivalenzprinzips vorzusehen. Dieser Ansatz induziert eine effiziente Bereitstellung von "privaten" Gütern durch den Staat und verhindert die Belastung anderer Individuen, da analog zum Marktprozeß eine individuelle Abwägung der jeweiligen Nutzen und Kosten erzwungen wird. Das Äquivalenzprinzip ermöglicht eine flexible Anpassung staatlicher Leistungen an die Wählerpräferenzen und sollte bei Vorliegen marktähnlicher Nachfragebeziehungen angewendet werden ${ }^{1}$ ).

In diesem Zusammenhang besteht die Möglichkeit einer Zweckbindung ${ }^{2)}$ einzelner Steuern für bestimmte Ausgabenkomponenten. Dieser Ansatz stellt auf die steuerlichen Implikationen von Ausgabemaßnahmen ab. Zielgerechte Zweckbindungen besitzen den Vorteil, daß sie die individuellen Präferenzen erfassen und dem Postulat der Konsumentensouveränität entsprechen. Eine Besteuerung von komplementären Gütern ist allerdings nur dann sinnvoll, wenn die Einnahmenentwicklung die Nachfrage nach der betreffenden staatlichen Leistung widerspiegelt ${ }^{3}$. Entwickelt sich das Steueraufkommen dagegen unabhängig von den Präferenzen für die staatliche Leistung, ist eine ineffiziente Bereitstellung der entsprechenden Leistung zu erwarten ${ }^{4}$ ).

1) Bei der Bereitstellung rein öffentlicher Güter ergeben sich allerdings Probleme, da die jeweiligen Steuerpreise entsprechend den unterschiedlichen marginalen Bewertungen der einzelnen Individuen bestimmt werden müssen. In dieser Konstellation besteht kein Anreiz zur Präferenzoffenbarung, so daß die Anwendung des Äquivalenzprinzips aufgrund des "Trittbrettfahrer"-Verhaltens der Steverzahler nicht möglich ist.

2) Vgl. dazu Wyrick (1989), S. $284 \mathrm{f}$.

3) Als Beispiel sei auf die Zweckbindung von Mineralöl-bzw. Kraftfahrzeugsteuer zur Finanzierung des Straßenbaus verwiesen.

4) Vgl. dazu Folkers (1983b), S. 34 . 
Eine wesentliche Ursache des Rent-Seeking liegt in der politisch opportunen Gewährung steuerlicher Sonderregelungen. Eine allgemeine Besteverung ohne Differenzierung nach Gütern bzw. Individuen kann die Vergabe ineffizienter Steuerprivilegien einschränken. Außerdem besteht die Notwendigkeit, eine umfassende Bemessungsgrundlage zu wählen und in Form einer quasi-permanenten Regel ${ }^{1}$ ) zu fixieren. Ausnahmen davon sind eng und präzise festzulegen, um keine Anreize zum Wettbewerb um Steuervorteile zu induzieren. Änderungen dieser Regeln sollten nur mit qualifizierter Mehrheit möglich sein, damit "die Schwelle für die Einführung gruppenspezifischer Sondervorteile höher wird"2).

Zur Ergänzung dieser Maßnahmen sollte das Volumen der durch Steververgünstigungen erzeugten Einnahmenverluste des States begrenzt werden. Beispielsweise besteht die Möglichkeit, den Umfang der Steververgünstigungen in Höhe eines bestimmten Prozentsatzes des Steueraufkommens festzulegen. Als übergangsregelung könnte eine proportionale Kürzung der bisherigen Vergünstigungen vereinbart werden ${ }^{3}$ ). Wird zusätzlich eine automatische Reduktion der Vergünstigung jährlich um einen fest vorgegebenen Prozentsatz verabschiedet, resultiert eine Einengung des Spielraums der politischen Entscheidungsträger bei der Realisierung von partikularen Steuervorteilen.

1) Vgl. Buchanan (1976), S. 27 .

2) Folkers (1985), S. 299.

3) Vgl. dazu auch den Sachverständigenrat zur Begutachtung der gesamtwirtschaftlichen Entwicklung, der sich für eine lineare Kürzung aller Finanzhilfen ausspricht. Trotz aller damit verbundenen Probleme "ist eine lineare Kürzung der Finanzhilfen besser als ein weiterhin ungehindertes Anschwellen der Subventionen". Jahresgutachten (1988/89), S. 150 . 
Ebenso sollten "heimliche" Steuererhöhungen ausgeschlossen werden, um eine Finanzierung von Sonderprivilegien ohne parlamentarischen Beschluß zu vermeiden. Die progressive Ausgestaltung des Steuersystems führt sowohl bei Wachstum als auch bei inflationären Einkommenssteigerungen automatisch zu überproportionalen Stevererhöhungen, die zu partikularen Begünstigungen verwendet werden können. Aus diesem Grund ist eine bindende Indexierungsregel erforderlich, die Steuererhöhungen ohne expliziten Beschluß des Parlamentes verbietet.

Im Wege der sogenannten "Sunset-Legislation"1) ist für einzelne Vergünstigungen eine gewisse Laufzeit vorzusehen, nach deren Ablauf über eine Verlängerung erneut abgestimmt werden muß. Dadurch ergibt sich die Notwendigkeit, über die Sinnhaftigkeit einer Aufrechterhaltung von einmal gewährten Privilegien zu entscheiden und deren Zweckmäßigkeit zu prüfen ${ }^{2}$ ). Die Abschaffung steuerlicher Privilegien ließe sich politisch eher durchsetzen, wenn sie nach einem fixen Zeitraum wegfielen bzw. nur durch expliziten Beschluß verlängert werden könnten.

Darüber hinaus reduziert eine Begrenzung der Einnahmeninstrumente des Staates auf einige, generelle Steuerquellen die Komplexität und Ergiebigkeit des Steuersystems, so daß eine politische Förderung von bestimmten Partikularinteressen eingeschränkt wird. Als Konsequenz dieser Maßnahmen wird eine verstärkte Privilegierung im Ausgaben- bzw. Regulierungsbereich angestrebt.

1) Vgl. dazu Folkers (1983b), S. 39 f. bzw. Gabriel (1983), S. $230 \mathrm{f}$.

2) Automatische Fortschreibungen ohne parlamentarischen Entscheid liegen im unmittelbaren Interesse der Politiker, weil auf diese Weise gruppenspezifische Vorteile gewährt werden können, ohne Stimmenverluste durch oppositionelle Gruppen hinnehmen zu müssen. 
Die intendierte Verminderung der Ressourcenverschwendung kann nur erreicht werden, wenn entsprechende Vorkehrungen auf der Ausgabenseite ${ }^{1)}$ getroffen werden. Eine wirksame prozedurale Beschränkungsmaßnahme stellt das Gebot des materiellen Budgetausgleichs dar. Diese Vorkehrung gegen den Einsatz des Finanzierungsinstrumentes "Staatsverschuldung" erschwert eine Verschleierungsstrategie im Hinblick auf die Opportunitätskosten politisch gewährter Privilegien. Damit werden die Möglichkeiten vermindert, abgegrenzte Gruppen gezielt zu begünstigen und die Kosten der Vorteilsgewährung möglichst breit bzw. unmerklich zu streven. Das Verbot der Kreditfinanzierung ${ }^{2}$ verstärkt den Widerstand gegen eine Erhöhung gruppenbezogener Staatsausgaben, so daß einer weiteren Zunahme der interessenorientierten Statstätigkeit entgegengewirkt wird.

Die Einführung der Budgetausgleichsvorschrift verlagert die Rent-Seeking-Aktivitäten der Interessengruppen in zunehmendem Maße in den Bereich staatlichen Handelns, der sich nicht im Budget niederschlägt ${ }^{3)}$. Die Bemühungen konzentrieren sich vor allem auf die Durchsetzung von vorteilhaften Regulierungen, die in ihren Wirkungen vielfach der staatlichen Ausgabenund Stevertätigkeit entsprechen ${ }^{4}$ ).

1) Vgl. dazu auch Folkers (1984b), S. $173 \mathrm{f}$.

2) Zur Berücksichtigung der Problematik intertemporaler Effizienz bzw. der Gerechtigkeit zwischen Generationen und der Konstellation von gravierenden nachfragebedingten Ungleichgewichten sind gewisse Zusatzvorschriften vorzusehen. Vgl. Folkers (1983b), S. 37 f.

3) Die Problematik sogenannter "Off-Budget-Aktivitäten" wird in grundsätzlicher Form bei Bennett/DiLorenzo (1983) behandelt. Im Rent-Seeking-Kontext sei auf Bennett/DiLorenzo (1984), S. 222 verwiesen.

4) Vgl. dazu Posner (1971), S. 22. 
"Direct taxing and spending on the one hand, and regulation on the other, thus become substitutable technologies"1). Regulierende Interventionen garantieren daverhaft Verteilungsvorteile zugunsten einzelner gesellschaftlicher Gruppen und sind besonders geeignet, die wahren Umverteilungsmotive politisch opportun zu verhüllen. In Analogie zur Vorgehensweise bei den Steuervergünstigungen kann auch in diesem Bereich das Verfahren der Sunset-Legislation angewendet werden ${ }^{2}$ ). Nach einem genau fixierten Zeitraum ist ein neverlicher parlamentarischer Beschluß notwendig, um bestimmte Regulierungen zu verlängern ${ }^{3}$ ).

Setzt sich die Erkenntnis durch, daß die Möglichkeiten für die Vergabe gruppenspezifisch begünstigender Vorhaben reduziert sind, wird auch der Ressourceneinsatz für Rent-Seeking wegen der geringeren Gewinnchancen zurückgehen ${ }^{4}$ ). Wenngleich diese Reformvorschläge isoliert betrachtet jeweils Umgehungsmöglichkeiten eröffnen, können sie in ihrem Zusammenwirken durchaus zu einer Verringerung der vielfätigen, distributiv motivierten Ausnahmeregelungen beitragen. Darüber hinaus sind alle Institutionen von Vorteil, die marktliche Aktivitäten ertragreich machen und damit die Substitution des "unproduktiven" Einsatzes von Ressourcen für Rent-Seeking durch die produktive Ressourcenverwendung für Profit-Seeking fördern ${ }^{5}$ ).

1) Aranson/Ordeshook (1981), S. 167.

2) Vgl. Aranson/Ordeshook (1981), S. 167. Diese Vorgehensweise ist allerdings problematischer als im dargestellten Fall der steuerlichen Vergünstigungen, bei dem eine Verlängerung von Steuervorteilen nur bei Reduzierung anderer steverlicher Privilegien erlaubt ist.

3) Zur Konstruktion eines "Regulierungsbudgets" sei auf DeMuth $(1980)$, S. 38 ff. verwiesen.

4) Vgl. allerdings auch Laux (1984), S. 169.

5) Vgl. dazu die Argumentation in Kap. II.3. dieser Arbeit. 


\subsection{Förderung marktlicher Aktivitäten}

Im Rahmen der Wirtschaftsordnungspolitik sollen Anreize zu verstärkten marktlichen Aktivitäten geschaffen werden. Dazu eignen sich Regeln, die eine Steigerung der individuellen Ertragschancen unternehmerischen Handelns bewirken und die Bereitschaft zur Übernahme marktlicher Risiken erhöhen. Neben einer Stärkung der privaten Verfügungsrechte ist insbesondere für eine Konstanz der Regeln zu sorgen, die langfristig marktliche Dispositionen ermöglichen sollen. Beispielsweise wirkt eine Verringerung der Grenzsteuersätze ${ }^{1)}$ leistungsfördernd, da der Grenzertrag des marginalen Ressourceneinsatzes in produktiver Verwendung steigt. Dies impliziert eine Erhöhung der Opportunitätskosten des Rent-Seeking und damit eine Reduktion der politischen Einflußnahmeaktivitäten.

Ebenso ist ein Abbau steuerlicher Diskriminierungen von unternehmerischen, risikoreichen Maßnahmen notwendig, um das Streben nach marktlichen Renten zu fördern. Eine Verminderung des Progressionsgrades der Einkommensteuer induziert Anreize zur verstärkten übernahme von marktlichen Risiken. Aktivitäten mit hohem Risiko sind in aller Regel mit höheren Einkommensfluktuationen verbunden als weniger risikobehaftete Tätigkeiten. Damit ergeben sich bei schwankenden Periodeneinkommen höhere Steuerbelastungen als bei gleichmäßig anfallenden Einkommensströmen. Mit einem verminderten Progressionsgrad wird die Diskriminierung der Bereitschaft zur Risikoübernahme reduziert ${ }^{2}$.

1) Vgl. Brennan/Buchanan (1980), S. 34 .

2) Bei Einbeziehung von Verlusten ergibt sich, daß die Progression der Einkommensteuer einen vollständigen Verlustausgleich verhindert. Vgl. z.B. Herb (1988), S. 100. 
Eine wirksame Wirtschaftsordnungspolitik soll Risiken "kalkulierbar" machen und Anpassungen an veränderte Marktkonstellationen fördern. Der notwendige Strukturwandel wird allerdings durch zahlreiche Bestimmungen des Steverrechts bzw. des Arbeits- und Sozialrechts verhindert. Diese Regelungen verlagern Risiken auf Unternehmer und vermindern Anreize für marktliches Handeln, da soziale Kosten unternehmensspezifisch internalisiert werden.

Mit der Reduktion gesicherter Privilegien in allen Bereichen staatlichen Handelns wird das Streben nach politischen Renten durch innovative marktliche Aktivitäten substituiert ${ }^{1)}$. Aufgrund der Tatsache, daß die Allokationsverluste mit zunehmender Steuerprogression ansteigen und die zahlreichen Ausnahmeregelungen nur unsystematische Verteilungswirkungen hervorrufen, besteht die Möglichkeit zur Durchführung entsprechender Reformen ${ }^{2}$. "Tax reform may become a 'possible dream' when it is recognized that after taxpayers have adjusted to loopholes, those who avail themselves of pecial rules and regulations are not materially better off than they would have been in the absence of such rules and regulations" ${ }^{3)}$. Die Diskussion der verschiedenen Vorschläge zur Änderung der institutionellen Bedingungen marktlichen und politischen Handelns wirft die Frage nach den Realisierungschancen derartiger Reformkonzepte $a \cup f^{4)}$.

1) Vgl. Buchanan (1987b), S. $31 \mathrm{f}$.

2) Brennan/Buchanan (1980), S. 31 ff. konstatieren eine proportionale Belastungswirkung der Vergünstigungen.

3) Brennan/Buchanan (1980), S. 34.

4) "Die Durchsetzung von mehr Markt bedarf aber wiederum der politischen Entscheidung." v. Arnim (1987), S. 25. 


\subsection{Realisierungschancen und Implementationsprobleme}

Während die Wirtschaftsordnungspolitik in erster Linie mit Blick auf das Verhalten von Dritten erfolgt, besteht das wesentliche Charakteristikum der Staatsordnungspolitik darin, daß die Politiker sich selbst bestimmten Regelungen unterwerfen müssen. Die Verwirklichung eines derartigen Reformkonzeptes hängt somit wesentlich von der Bereitschaft der Politiker zur "Selbstbindung" ab. Da die abgeleiteten institutionellen Änderungen den Interessen der Politiker zuwiderlaufen, wird die Staatsordnungspolitik "zur Schwachstelle jeder auf Zweckrationalität gerichteten Wirtschaftsordnungspolitik"1). Die Realisierungschancen für Reformen ${ }^{2}$ ) steigen, wenn die ökonomischen Fehlentwicklungen weiter zunehmen. Wachsende Unzufriedenheit bei den Wählern aufgrund hoher Belastungen fördert die Bereitschaft von Politikern, entsprechende Forderungen in ihr politisches Programm aufzunehmen, um Wählerstimmen zu gewinnen. Die Implementierung der entwickelten Regeln wird begünstigt, wenn es gelingt, den für alle förderlichen Effekt zu verdeutlichen ${ }^{3)}$. Der Abbau der vielfältigen gruppenspezifischen Sonderregelungen bewirkt hohe Allokationsgewinne, die für Kompensationszwecke zur Verfügung stehen ${ }^{4}$. Es ist somit nicht zuletzt von der wirtschaftlichen und politischen Situation abhängig, inwieweit die genannten Reformvorschläge eine Chance erhalten.

1) Cassel (1988), S. 322 .

2) "Reform in the rent-seeking society depends critically on the history of its evolution, as well as upon the ability of political and intellectual leaders to think in terms of, and be persuasive about, general constitutional changes in the whole structure of social and economic institutions." Buchanan (1980c), S. 367 .

3) Vgl. Koford (1984), S. 207 bzw. Tullock (1988d), S. 472 ff.

4) Vgl. Buchanan (1980c), S. 366 . 


\section{Zusammenfassung}

Die Analyse der Vorteilsgewinnung im Wege des Rent-Seeking über den politischen Sektor führt zur Entwicklung von Reformvorschlägen, die eine Neuorientierung der Ordnungspolitik implizieren. Die ständige Ausdehnung staatlicher Interventionen im Zuge des Rent-Seeking und die damit verbundenen sozialen Verluste sind das Resultat bestehender Institutionen. Damit ergibt sich die Notwendigkeit zu institutionellen Reformen, die auf Verhaltensänderungen der am Rent-SeekingProzeß beteiligten Akteure abzielen.

Das Konzept der Staatsordnungspolitik verfolgt das Ziel, den wirtschafts- und finanzpolitischen Handlungsspielraum der Politiker bei der Vergabe gruppenspezifischer Verteilungsvorteile einzuengen. Institutionelle Vorgaben für das politische Entscheidungsverfahren erlauben eine Begrenzung der negativen ökonomischen Konsequenzen des Rent-Seeking, ohne die Möglichkeiten für sinnvolle und notwendige Interventionen des Staates zu beeinträchtigen. Die Aufgabe der Wirtschaftsordnungspolitik besteht darin. Anreize für einen verstärkten marktlichen Ressourceneinsatz der Individuen zu induzieren. Dazu sind Regeln geeignet, die zur Steigerung der Ertragschancen unternehmerischer Aktivitäten beitragen bzw. die Bereitschaft zur Übernahme marktlicher Risiken erhöhen.

Durch die Konstruktion wirksamer Restriktionen für den politischen und den marktlichen Prozeß werden die Voraussetzungen dafür geschaffen, daß die Funktionsfähigkeit des marktwirtschaftlichen Ordnungssystems in demokratischen Staatswesen erhalten bleibt. Eine derartige Ordnungspolitik kann Politiker und Wirtschaftssubjekte zu einem Verhalten veranlassen, das die allokativen Verluste vermindert und gesellschaftlich erwünschte Ergebnisse ermöglicht. 


\section{SCHLUSSBEMERKUNGEN}

Die seit einigen Jahren in der Entwicklung begriffene Theorie des Rent-Seeking stellt einen Erklärungsansatz für das Zustandekommen statlich determinierter Institutionen in demokratischen Gesellschaftssystemen dar. Das Rent-Seeking-Konzept beruht auf der Hypothese, daß sich mit dem Wandel vom liberalen Rechtsstaat zum Sozialstaat moderner Prägung vielfätige Möglichkeiten zur interessenbezogenen Ausgestaltung von staatlichen Regelungen eröffnen. Die an Partikularinteressen orientierte Ausrichtung der institutionellen Rahmenbedingungen ist charakteristisch für eine "Rent-Seeking-Society".

Durch die ständige Ausdehnung distributiv motivierter staatlicher Maßnahmen ergeben sich Anreize, Einfluß auf den politischen Entscheidungsprozeß auszü̈ben und die staatliche Zwangsgewalt für die Durchsetzung spezieller Sonderinteressen zu nutzen. Zu diesem Zweck werden Interessengruppen gebildet, die um die gruppenspezifisch begünstigende Ausgestaltung der Ordnungspolitik in marktwirtschaftlichen Systemen konkurrieren. Der Interessenwettbewerb um Verteilungsvorteile über den politischen Sektor nimmt umfangreiche Ressourcen in Anspruch, die alternativen produktiven Verwendungen entzogen werden. Im Widerstreit der Gruppeninteressen wird der Stat in zunehmendem Maße in Verteilungskonflikte verstrickt und begibt sich in die Abhängigkeit von Einzelinteressen. Die von Wählerstimmen abhängigen Politiker werden sich für gruppenspezifisch begünstigende Regelungen entscheiden, um bestimmte Wählergruppen zu erhalten bzw. zu gewinnen. Der Hinweis auf die Verwirklichung sozialer Zielsetzungen erleichtert in der öffentlichkeit die Rechtfertigung von partikularen Sondervorteilen und ermöglicht eine politisch opportune Verschleierung der wahren Unverteilungsmotive. 
Das grundlegende Problem einer "Sozialen Marktwirtschaft" besteht darin, ein ausgewogenes Verhältnis zwischen dem Prinzip der Freiheit am Markt und dem Prinzip des sozialen Ausgleichs zu finden. Der Staat hat eine Grundsicherung für soziale Notfälle zu übernehmen, jedoch nicht die marktlichen Institutionen zu verändern, um speziellen Gruppen Verteilungsvorteile zu garantieren. Anstatt die Ursachen bestimmter ökonomischer Fehlentwicklungen - nämlich unzweckmäßige staatliche Interventionen - zu beseitigen, werden immer neve punktuelle Eingriffe vorgenommen, ohne daß die angestrebten Zielsetzungen erreicht werden. Interessenvertretungen aller Art werden ermutigt, mit weiteren Gruppenforderungen an den Staat heranzutreten. Auf diese Weise werden vielgestaltige Möglichkeiten wechselseitiger Begünstigungen einzelner Interessengruppen zu Lasten unorganisierter Gruppeninteressen geschaffen. Die distributiv motivierten Interventionen in den Marktprozeß implizieren eine Verringerung der Flexibilität des marktlichen Anpassungsmechanismus und bilden damit die Voraussetzung für weitere staatliche Eingriffe. Daraus resultiert eine Ausdehnung des öffentlichen Sektors in marktwirtschaftlichen Wirtschaftssystemen.

Mit der Entwicklung zur "Rent-Seeking-Society" droht die Gefahr, daß mit dem ökonomischen System auch das politische System erstarrt, wenn der fortschreitenden Verlagerung von Distributionsentscheidungen vom marktlichen in den staatlichen Sektor keine Grenzen gesetzt werden. Diese Schlußfolgerung resultiert aus der Erkenntnis, daß der demokratische Prozeß bei der Lösung von Verteilungskonflikten überfordert ist. Zur Vermeidung der negativen ökonomischen Konsequenzen des Rent-Seeking-Verhaltens ist eine wirksame Ordnungspolitik notwendig, die sich langfristig auf die Gestaltung von verhaltensstevernden Institutionen konzentriert und den kurzfristig orientierten staatlichen Interventionismus einschränkt. 
Aufgrund der wechselseitigen Abhängigkeit von politischem Entscheidungsverhalten und distributiven Gruppenforderungen müssen die institutionellen Bedingungen ökonomischen Handelns im marktlichen und politischen Sektor in ihrem Zusammenwirken betrachtet werden. Wenngleich die Entwicklung eines Ordnungskonzepts für den öffentlichen Sektor noch am Anfang steht, sollte die seit langem allgemein akzeptierte Notwendigkeit einer Ordnungspolitik für den Marktprozeß auch für den politischen Prozeß nicht länger als Tabu betrachtet werden. Die Neuorientierung des ordnungstheoretischen Denkens ist notwendig, um den wirtschafts- und gesellschaftspolitischen Problemen in sozialstaatlichen Demokratien gerecht zu werden. 


\section{LITERATURVERZEICHNIS}

Abrams, B.A., Lewis, K.A. (1987)

A Median-Voter Model of Economic Regulation, in: Public Choice 52, S. 125-142

Addison, J.T., Genosko, J., Schnabel, C. (1989)

Gewerkschaften, Produktivität und Rent Seeking, in:

Jahrbuch für Nationalökonomie und Statistik, Bd. 206,

S. $102-116$

Akerlof, G.A. (1970)

The Market for Lemons: Quality Uncertainty and the Market Mechanism, in: Quarterly Journal of Economics 84 ,

S. $488-500$

Albert, H. (1977)

Individuelles Handeln und soziale Steverung. Die ökonomische Tradition und ihr Erkenntnisprogramm, in: H. Lenk, (Hrsg.), Handlungstheorien - interdisziplinär, Bd. 4, München, S. 177-225

Alchian, A.A. (1987)

Rent, in: J. Eatwell, M. Milgate, P. Newman (Hrsg.),

The New Palgrave, Bd. 4, London U.a.0., S. 141-143

Allard, R.J. (1988)

Rent-Seeking with Non-Identical Players, in: Public

Choice 57 , S. 3-14

Alt, J.E. (1983)

The Evolution of Tax Structures, in: Public Choice 41, S. 181-222

Anam, M. (1982)

Distortion-Triggered Lobbying and Welfare - A Contribution to the Theory of Directly-Unproductive Profit-Seeking Activities, in: Journal of International Economics 13, S. 15-32

Andel, N. (1984)

Zum Konzept der meritorischen Güter, in: Finanzarchiv N.F. 42 , S. 630-648

Anderson, G.M. (1987)

Welfare Programs in the Rent-Seeking Society, in:

Southern Economic Journal 54, S. 377-386 


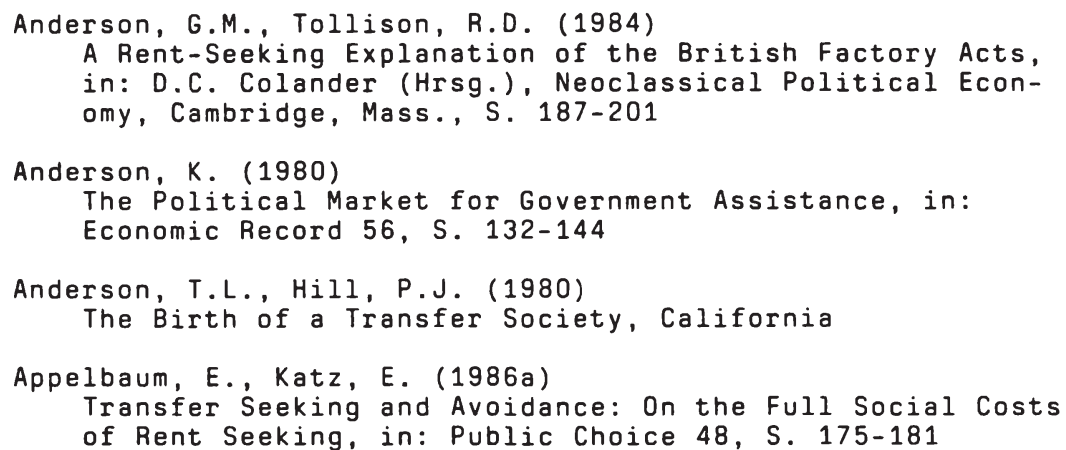

Appelbaum, E., Katz, E. (1986b)

Rent-Seeking and Entry, in: Economics Letters 20, S. 207-212

Appelbaum, E., Katz, E. (1987)

Seeking Rents by Setting Rents: The Political Economy of

Rent Seeking, in: Economic Journal 97, S. 685-699

Aranson, P.M., Ordeshook, P.C. (1981)

Alternative Theories of the Growth of Government and Their Implications for Constitutional Tax and Spending

Limits, in: H.F. Ladd, N.T. Tideman (Hrsg.), Tax and

Expenditure Limitations; Washington, D.C., S. 143-189

v. Arnim, H.H. (1987)

Staatsversagen: Schicksal oder Herausforderung?, in: Aus Politik und Zeitgeschichte. Beilage zur Wochenzeitung das Parlament, B 48, S. 17-28

Arrow, K.J. (1963)

Social Choice and Individual Values, 2. Auflage, New Haven

Arrow, K.J. (1970)

Essays in the Theory of Risk-Bearing, Amsterdam/London

Atkinson, A.B., Stiglitz, J.E. (1980)

Lectures on Public Economics, Maidenhead/Berkshire

Axelrod, R. (1984)

The Evolution of Cooperation, New York

Baldwin, R.E. (1984)

Rent-Seeking and Trade Policy: An Industry Approach, in: Weltwirtschaftliches Archiv 120, S. 662-677 
Balisacan, A.M., Roumasset, J.A. (1987)

Public Choice of Economic Policy: The Growth of

Agricultural Protection, in: Weltwirtschaftliches Archiv 123 , S. 232-248

Barro, R.J. (1973)

The Control of Politicians: An Economic Model, in:

Public Choice 14, S. 19-42

Bartlett, R. (1973)

Economic Foundations of Political Power, New York/London

Bastiat, F. (1854)

Sophismes Économiques, Paris

Bator, F. (1958)

The Anatomy of Market Failure, in: Quarterly Journal of Economics 72, S. 351-379

Baumol, W.J., Bradford, D.F. (1970)

Optimal Departures from Marginal Cost Pricing, in:

American Economic Review 60, S. 265-283

Baysinger, B., Ekelund Jr., R.B., Tollison, R.D. (1980)

Mercantilism as a Rent-Seeking Society, in: J.M.

Buchanan, R.D. Tollison, G. Tullock (Hrsg.), Toward a

Theory of the Rent-Seeking Society, Texas, S. 235-268

Becker, G.S. (1965)

A Theory of the Allocation of Time, in: Economic Journal 75 , S. $493-517$

Becker, G.S. (1976)

The Economic Approach to Human Behavior, Chicago/London

Becker, G.S. (1981)

A Treatise on the Family, Cambridge

Becker, G.S. (1983)

A Theory of Competition among Pressure Groups for

Political Influence, in: Quarterly Journal of Economics 98 , S. 371-400

Becker, G.S. (1985)

Public Policies, Pressure Groups, and Dead Weight Costs, in: Journal of Public Economics 28, S. 329-347

Bennett, J.T., DiLorenzo, Th.J. (1983)

Underground Government: The Off-Budget Public Sector, Washington, D.C. 
Bennett, J.T., DiLorenzo, Th.J. (1984)

Political Entrepreneurship and Reform of the Rent-

Seeking Society, in: D.C. Colander (Hrsg.), Neoclassical

Political Economy, Cambridge, Mass., S. 217-227

Benson, B.L. (1984)

Rent Seeking from a Property Rights Perspective, in:

Southern Economic Journal 51, S. 388-400

Benson, B.L., Faminow, M.D. (1988)

Location Choice and Urban Growth in a Rent-Seeking

Society, in: Public Finance Quarterly 16, S. 158-177

Benson, B.L., Mitchell, J.M. (1988)

Rent Seekers Who Demand Government Production: Bureaucratic Output and the Price of Complements, in: Public Choice 56, S. 3-16

Bentley, A.F. (1908)

The Process of Government, Chicago

Bernholz, P. (1969)

Einige Bemerkungen zur Theorie des Einflusses der Verbände auf die politische Willensbildung in der Demokratie, in: Kyklos 22 , S. 276-288

Bernholz, P. (1973)

Die Machtkonkurrenz der Verbände im Rahmen des politischen Entscheidungssystems, in: H.K. Schneider, Ch. Watrin (Hrsg.), Macht und ökonomisches Gesetz, Berlin, S. $859-881$

Bernholz, P. (1974)

On the Reasons of the Influence of Interest Groups of Political Decision-Making, in: Zeitschrift für Wirtschafts- und Sozialwissenschaften 94, S. 45-63

Bernholz, P. (1975)

Grundlagen der politischen Ökonomie, Bd. 2, Tübingen

Bernholz, P., Breyer, F. (1984)

Grundlagen der Politischen ökonomie, 2. Auflage, Tübingen

v. Beyme, K. (1980)

Interessengruppen in der Demokratie, 5. Auflage, München

Bhagwati, J.N. (1980)

Lobbying and Welfare, in: Journal of Public Economics 14 , S. 355-363 
Bhagwati, J.N. (1982a)

Directly Unproductive Profit-Seeking (DUP) Activities, in: Journal of Political Economy 90, S. 988-1002

Bhagwati, J.N. (1982b)

Lobbying, DUP Activities, and Welfare: A Response to

Tullock, in: Journal of Public Economics 14, S. 395-401

Bhagwati, J.N. (1983)

DUP Acitvities and Rent Seeking, in: Kyklos 36 ,

S. 634-637

Bhagwati, J.N. (1987)

Directly Unproductive Profit-Seeking (DUP) Activities, in: J. Eatwell, M. Milgate, P. Newman (Hrsg.), The New Palgrave, Bd. 1, London U.a.0., S. 845-846

Bhagwati, J.N., Brecher, R.A., Srinivasan, T.N. (1984) DUP Activities and Economic Theory, in: D.C. Colander (Hrsg.), Neoclassical Political Economy, Cambridge, Mass., S. 17-32

Bhagwati, J.N., Srinivasan, T.N. (1982)

The Welfare Consequences of Directly Unproductive

Profit-Seeking (DUP) Lobbying Activities, in: Journal of International Economics 13, S. 33-44

Black, D. (1948)

On the Rationale of Group Decision Making, in: Journal of Political Economy 56, S. 23-34

Blankart, Ch.B. (1983)

Warum wächst der Sozialstaat? Eine institutionelle Erklärung, in: P. Koslowski, Ph. Kreuzer (Hrsg.), Chancen und Grenzen des Sozialstaates, Tübingen, S. 154-160

Blümle, E.-B., Schwarz, P. (1985)

Wirtschaftsverbände und ihre Funktion, Darmstadt

Boadway, R.W., Wildasin, D.E. (1984)

Public Sector Economics, 2. Auflage, Boston/Toronto

Böbel, I. (1988)

Eigentum, Eigentumsrechte und institutioneller Wandel, Berlin U.a.o.

Borcherding, Th.E. (1977)

Budgets and Bureaucrats: The Sources of Government Growth, Durham

Brennan, G. (1973)

Pareto Desirable Redistribution: The Nonaltruistic

Dimension, in: Public Choice 14, S. 43-67 
Brennan, G., Buchanan, J.M. (1980/1988)

The Power to Tax, Cambridge u.a.0.

(deutsche übersetzung: Besteverung und Staatsgewalt, Hamburg)

Brennan, G., Buchanan, J.M. (1981)

Der verteilende Staat: Ansätze zu einer Theorie der

Umverteilung, in: Zeitschrift für Wirtschaftspolitik 30 , S. 103-128

Brennan, G., Buchanan, J.M. (1985)

The Reason of Rules, Cambridge u.a.0.

Brennan, G., Lomasky, L. (1983)

Institutional Aspects of "Merit Goods" Analysis, in:

Finanzarchiv N.F. 41, S. 183-206

Brennan, G., McGuire, Th. (1975)

Optimal Policy Choice under Uncertainty, in: Journal of

Public Economics 4, S. 205-209

Brennan, G., Tollison, R.D. (1980)

Rent-Seeking in Academia, in: J.M. Buchanan, R.D.

Tollison, G. Tullock (Hrsg:), Toward a Theory of the

Rent-Seeking Society, Texas, S. 344-356

Breton, A. (1974)

The Economic Theory of Representative Government,

Chicago

Brock, W.A., Magee, St.P. (1984)

The Invisible Foot and the Waste of Nations: Redistribution and Economic Growth, in: D.C. Colander (Hrsg.), Neoclassical Political Economy, Cambridge, Mass.,

S. $177-185$

Brooks, M.A., Heijdra, B.J. (1987)

Rent-Seeking and Pollution Taxation: An Extension, in:

Southern Economic Journal 54, S. 335-342

Brooks, M.A., Heijdra, B.J. (1988)

In Search of Rent-Seeking, in: Ch.K. Rowley, R.D.

Tollison, G. Tullock (Hrsg.), The Political Economy of

Rent-Seeking, Boston, S. 27-49

Browning, E.K. (1974)

On the Welfare Cost of Transfers, in: Kyklos 2,

S. 374-377

Browning, E.K., Browning, J.M. (1983)

Microeconomics Theories and Applications, Boston/

Toronto 
Brunner, K. (1978)

Reflections on the Political Economy of Government: The

Persistent Growth of Government, in: Schweizerische

Zeitschrift für Volkswirtschaft und Statistik 114,

S. 649-680

Buchanan, J.M. (1966)

Externality in Tax Response, in: Southern Economic Journal 33, S. 35-42

Buchanan, J.M. (1967)

Public Finance in Democratic Process, Chapel Hill

Buchanan, J.M. (1969)

Cost and Choice, Chicago/London

Buchanan, J.M. (1975)

The Limits of Liberty, Chicago/London

(deutsche Übersetzung: Die Grenzen der Freiheit,

Tübingen, 1984)

Buchanan, J.M. (1976)

Taxation in Fiscal Exchange, in: Journal of Public

Economics 6, S. 17-29

Buchanan, J.M. (1978)

The Public Sector versus the Private Sector in a Market Economy, Seminar Presentation, Centro de Estudios y Communicacion Economica, Madrid

Buchanan, J.M. (1980a)

Rent Seeking and Profit Seeking, in: J.M. Buchanan, R.D. Tollison, G. Tullock (Hrsg.), Toward a Theory of the Rent-Seeking Society, Texas, S. 3-15

Buchanan, J.M. (1980b)

Rent Seeking under External Diseconomies, in: J.M.

Buchanan, R.D. Tollison, G. Tullock (Hrsg.), Toward a Theory of the Rent-Seeking Society, Texas, S. $183-194$

Buchanan, J.M. (1980c)

Reform in the Rent-Seeking Society, in: J.M. Buchanan, R.D. Tollison, G. Tullock (Hrsg.), Toward a Theory of the Rent-Seeking Society, Texas, S. 359-367

Buchanan, J.M. (1983)

Rent Seeking, Noncompensated Transfers, and Laws of Succession, in: Journal of Law and Economics 26. S. $71-85$ 
Buchanan, J.M. (1987a)

Constitutional Economics, in: J. Eatwell, M. Milgate, P. Newman (Hrsg.). The New Palgrave, Bd. 1, London U.a.0., S. 585-588

Buchanan, J.M. (1987b)

Tax Reform as Political Choice, in: Economic Perspectives 1 , S. 29-35

Buchanan, J.M., Brennan, G. (1980)

Tax Reform without Tears, in: H.J. Aaron, M.J. Boskin, (Hrsg.), The Economics of Taxation, Washington, D.C., S. $33-53$

Buchanan, J.M., Flowers, M. (1969)

An Analytical Setting for a "Taxpayers' Revolution", in: Western Economic Journal 7, S. 349-359

Buchanan, J.M., Pauly, M.V. (1970)

On the Incidence of Tax Deductibility, in: National Tax Journal 23, S. 157-167

Buchanan, J.M., Tullock, G. (1962)

The Calculus of Consent. Ann Arbor

Buchanan, J.M., Vanberg, V. (1988)

The Politicization of Market Failure, in: Public Choice 57. S. 101-113

Bush, W.C., Denzau, A.T. (1977)

The Voting Behavior of Bureaucrats and Public Sector Growth, in: Th.E. Borcherding (Hrsg.), Budgets and Bureaucrats: The Sources of Government Growth, Durham, S. $90-99$

Cassel, D. (1988)

Wirtschaftspolitik als Ordnungspolitik, in: D. Cassel, B.-Th. Ramb, H.J. Thieme (Hrsg.), Ordnungspolitik, München, S. 313-333

Coase, R.H. (1984)

The New Institutional Economics, in: Zeitschrift für die gesamte Staatswissenschaft (Journal of Institutional and Theoretical Economics) 140, S. 229-231

Coe, R.D., Wilber, Ch.K. (1985)

Schumpeter Revisited: An Overview, in: R.D. Coe, Ch.K. Wilber (Hrsg.), Capitalism and Democracy: Schumpeter Revisited, Indiana, S. 1-59 
Colander, D.C. (1985)

Some Simple Geometry of the Welfare Loss from Competi-

tive Monopolies, in: Public Choice 45, S. 199-206

Colander, D.C., Olson, M. (1984)

Coalitions and Macroeconomics, in: D.C. Colander (Hrsg.), Neoclassical Political Economy, Cambridge, Mass.,

S. $115-128$

Coleman, J.S. (1972)

Collective Decisions and Collective Action, in:

P. Laslett, P.W. Runciman, Q. Skinner (Hrsg.),

Philosophy, Politics and Society, Oxford, S. 208-219

Congleton, R.D. (1980)

Competitive Process, Competitive Waste, and Institutions, in: J.M. Buchanan, R.D. Tollison, G. Tullock (Hrsg.), Toward a Theory of the Rent-Seeking Society, Texas, S. 153-179

Congleton, R.D. (1984)

Committees and Rent-Seeking Effort, in: Journal of

Public Economics 25, S. 197-209

Congleton, R.D. (1986)

Rent-Seeking Aspects of Political Advertising, in:

Public Choice 49, S. 249-263

Congleton, R.D. (1988)

Evaluating Rent-Seeking Losses: Do the Welfare Gains

of Lobbyists Count?, in: Public Choice 56, S. 181-184

Corcoran, W.J. (1984)

Long-Run Equilibrium and Total Expenditures in Rent-

Seeking, in: Public Choice 43, S. 89-94

Corcoran, W.J., Karels, G.V. (1985)

Rent-Seeking Behavior in the Long-Run, in: Public

Choice 46, S. 227-246

Coughlin, P.J., Mueller, D.C., Murrell, P. (1986)

Electural Politics, Interest Groups, and the Size of Government, Discussion Paper, Nr. 106, Monash University, Clayton, Victoria

Cowling, K., Mueller, D.C. (1978)

The Social Costs of Monopoly Power, in: Economic Journal 88 , S. 727-748

Crain, W.M., Shughart II, W.F., Tollison, R.D. (1988)

Voters as Investors: A Rent-Seeking Resolution of the

Paradox of Voting, in: Ch.K. Rowley, R.D. Tollison, G.

Tullock (Hrsg.), The Political Economy of Rent-Seeking, Boston, S. 241-249 
Crain, W.M., Zardkoohi, A. (1980)

$X$-Inefficiency and Nonpecuniary Rewards in a Rent-

Seeking Society: A Neglected Issue in the Property

Rights Theory of the Firm, in: American Economic

Review 70, S. 784-792

Crew, M.A. (1987)

Rent-Seeking Is Here to Stay, in: Ch.K. Rowley (Hrsg.),

Democracy and Public Choice, Oxford/New York, S. 158-162

Crew, M.A., Rowley, Ch.K. (1988)

Toward a Public Choice Theory of Monopoly Regulation, in: Public Choice 57, S. 49-67

Currie, J.M., Murphy, J.A., Schmitz, A. (1971)

The Concept of Economic Surplus and Its Use in Economic Analysis, in: Economic Journal 81, S. 741-799

Demsetz, H. (1984)

Purchasing Monopoly, in: D.C. Colander (Hrsg.), Neoclassi-

cal Political Economy, Cambridge, Mass., S. 101-113

DeMuth, Ch.C. (1980)

The Regulatory Budget, in: Regulation 4, S. 29-44

Denzau, A.T., Munger, M.C. (1983)

Legislators and Interest Groups: How Unorganized Interests Get Represented, Working Paper, $\mathrm{Nr}$. 81, Washington University, St. Louis, Missouri

Dilorenzo, Th.J. (1984)

The Domain of Rent Seeking Behaviour: Private or Public Choice?, in: International Review of Law and Economics 4, S. 185-197

DiLorenzo, Th.J. (1988)

Property Rights, Information Costs, and the Economics of Rent-Seeking, in: Zeitschrift für die gesamte Staatswissenschaft (Journal of Institutional and Theoretical Ecomomics) 144 , S. 318-332

Dnes, A.W. (1985)

Rent Seeking Behaviour and Open Access Fishing, in:

Scottish Journal of Political Economy 32, S. 159-170

Downs, A. (1957)

An Economic Theory of Political Action in a Democracy, in: Journal of Political Economy 65, S. 135-150

Downs, A. (1968)

ökonomische Theorie der Demokratie, Tübingen 
Ekelund Jr., R.B., Tollison, R.D. (1981)

Mercantilism as a Rent-Seeking Society: Economic Regulation in Historical Perspective, Texas

Ekelund Jr., R.B., Tollison, R.D. (1984)

A Rent-Seeking Theory of French Mercantilism, in: J.M. Buchanan, R.D. Tollison (Hrsg.), The Theory of Public Choice-II, Ann Arbor, S. 206-223

Ekelund Jr., R.B., Tollison, R.D. (1986)

Economics, Boston/Toronto

Elsner, W. (1986)

Ökonomische Institutionenanalyse, Berlin

Eschenburg, R. (1975)

Politische Unternehmer und öffentliche Güter, in:

E. Arndt (Hrsg.), Wirtschaft und Gesellschaft,

Tübingen, S. 257-302

Eucken, W. (1959)

Grundlagen der Nationalökonomie, 7. Auflage, Berlin

Eucken, W. (1975)

Grundsätze der Wirtschaftspolitik, 5. Auflage, Tübingen

Fabella, R.V. (1989)

Uncertain Monopoly Rent and the Social Cost of Rent

Seeking, in: Economics Letters 29, S. 199-203

Faith, R.L. (1980)

Rent-Seeking Aspects of Bureaucratic Competition, in:

J.M. Buchanan, R.D. Tollison, G. Tullock (Hrsg.),

Toward a Theory of the Rent-Seeking Society, Texas, S. $332-343$

Faminow, M.D., Benson, B.L. (1984)

Rent-Seeking and Supply Management in Canadian Agri-

culture, in: Canadian Journal of Agricultural Economics 32 , S. 548-559

Feldstein, M.S. (1976)

Compensation in Tax Reform, in: National Tax Journal

29 , S. $123-130$

Findlay, R., Wellisz, St. (1983)

Some Aspects of the Political Economy of Trade Restrictions, in: Kyklos 36, S. 469-481 
Findlay, R., Wellisz, St. (1984)

Toward a Model of Endogenous Rent-Seeking, in: D.C.

Colander (Hrsg.), Neoclassical Political Economy,

Cambridge, Mass., S. 89-100

Fleischmann, G. (1974)

Ungleichheit unter den Wählern - Zur ökonomischen Perspektive in der Soziologie, in: H.P. Widmaier (Hrsg.), Politische Ökonomie des Wohlfahrtsstaates, Frankfurt a.M.

Flowers, M.R., Stroup, R. (1979)

Coupon Rationing and Rent-Seeking Bureaucrats, in:

Public Choice 34, S. 473-479

Folkers, C. (1979)

Staatswirtschaftliche Quoten und Beziehungszahlen, in:

Wirtschaftsstudium 8 , S. 405-409 und 457-463

Folkers, C. (1983a)

Zu einer positiven Theorie der Steverreform, in: K. $-H$. Hansmeyer (Hrsg.), Staatsfinanzierung im Wandel,

Berlin, S. 189-211

Folkers, C. (1983b)

Begrenzungen von Steuern und Staatsausgaben in den USA. Eine Untersuchung über Formen, Ursachen und Wirkungen vorgeschlagener und realisierter fiskalischer Restriktionen, Baden-Baden

Folkers, C. (1984a)

Fiskalische Begrenzungen, in: Wirtschaftswissenschaftliches Studium 13, S. 500-506

Folkers, C. (1984b)

Konzepte zur Begrenzung von Steuern und Staatsausgaben, in: H.H. v. Arnim, K. Littmann (Hrsg.), Finanzpolitik im Umbruch: Zur Konsolidierung öffentlicher Haushalte, Berlin, S. 163-179

Folkers, C. (1985)

Steverreforminteressen und Steververgünstigungen in positiver und institutioneller Perspektive, in: Jahrbuch für Sozialwissenschaft, Bd. 36, S. 274-301

Folkers, C. (1986a)

Die Politische ökonomie der Besteverung, Diskussionsbeitrag, $\mathrm{Nr}$. 30/1986, Universität Hohenheim

Folkers, C. (1986b)

Das Dilemma des Defizits. Haushaltsentwicklung und gesetzliches Konsolidierungsverfahren in den USA, in: Finanzarchiv N.F. 44, S. 365-403 
Folkers, C. (1987)

Wandlungen der Verbrauchsbesteuerung, in: K. Häuser

(Hrsg.), Wandlungen der Besteuerung, Berlin, S. 89-212

Folkers, C. (1988)

Wer wird durch Steuervergünstigungen eigentlich begünstigt?, in: Finanzarchiv N.F. 46, S. 214-235

Formby, J.P., Keeler, J.P., Thistle, P.D. (1988) $X$-Efficiency, Rent Seeking and Social Costs, in: Public Choice 57, S. 115-126

Formby, J.P., Millner, E.L. (1987)

"Comparable Worth" and Rent Seeking, in: International

Review of Law and Economics 7, S. 65-78

Forte, F., Peacock, A. (1981)

Tax Planning, Tax Analysis and Tax Policy, in: A.

Peacock, F. Forte (Hrsg.), The Political Economy of

Taxation, 0xford, S. 3-28

Fratianni, M., Spinelli, F. (1982)

The Growth of Government in Italy: Evidence from 1861

to 1979, in: Public Choice 39, S. 221-243

Frey, B.S. (1971)

Why Do High Income People Participate More in Politics?, in: Public Choice 11, S. 101-105

Frey, B.S. (1972)

Political Participation and Income Level: An Exchange, in: Public Choice 13, S. 119-122

Frey, B.S. (1985)

Internationale Politische Ökonomie, München

Friedland, R., Sanders, J. (1985)

The Public Economy and Economic Growth in Western Market Economies, in: American Sociological Review 50, S. $421-437$

Frohlich, N.J., Oppenheimer, J.A., Young, 0. (1971)

Political Leadership and Collective Goods, Princetown

Furubotn, E.G., Pejovich, S. (1972)

Property Rights and Economic Theory: A Survey of Recent

Literature, in: Journal of Economic Literature 10,

S. 1137-1162 
Furubotn, E.G., Richter, R. (1984)

The New Institutional Economics, in: Zeitschrift für die gesamte Staatswissenschaft (Journal of Institutional and Theoretical Economics) 140, S. 1-6

Gabriel, L. (1983)

Das Wachstum des Staatssektors, Köln

Gabriel, L., Loderer. C. (1988)

Political Process and Government Growth, in: Zeitschrift für die gesamte Staatswissenschaft (Journal of Institutional and Theoretical Economics) 144, S. 267-295

Gäfgen, G. (1983)

Institutioneller Wandel und ökonomische Erklärung, in: E. Boettcher, Ph. Herder-Dorneich, K.-E. Schenk (Hrsg.), Jahrbuch für Neue Politische Ökonomie, Bd. 2, Tübingen

Gäfgen, G. (1984)

Entstehung und Expansion des Wohlfahrtsstaates, in:

Hamburger Jahrbuch für Wirtschafts- und Gesellschaftspolitik 29, S. 55-72

Gäfgen, G. (1987)

Kooperative Wirtschaftspolitik, never Korporatismus und Wirtschaftsordnung, Diskussionsbeitrag, $\mathrm{Nr} .230$, Universität Konstanz

Galbraith, J.K. (1956)

American Capitalism. The Concept of Countervailing

Power, 2. Auflage, Boston

Gantner, M. (1984)

Meßprobleme öffentlicher Aktivitäten, Baden-Baden

Gerstenberger, W., Heinze, J., Hummel, M., Sprenger, R.-U. (1984)

Staatliche Interventionen, Ergänzungsband zur Ifo-Strukturberichterstattung 1983, Ifo-Institut für Wirtschaftsforschung e.V., München

Goetz, Ch.J. (1964)

A Variable-Tax Model of Intersectoral Allocation, in:

Public Finance 19, S. 29-43

Goetz, Ch.J. (1977)

Fiscal Illusion in State and Local Finance, in: Th.E. Borcherding (Hrsg.), Budgets and Bureaucrats: The Sources of Government Growth, Durham, S. 176-187 
Goetz, M.L. (1978)

Tax Avoidance, Horizontal Equity, and Tax Reform: A Proposed Synthesis, in: Southern Economic Journal 44, S. 798-812

Goldberg, V.P. (1974)

Institutional Change and Quasi-Invisible Hand, in: Journal of Law and Economics 17, S. 461-492

Grampp, W.D. (1989)

Rent-Seeking in Arts Policy, in: Public Choice 60, S. 113-121

Groser, M. (1980)

Konzertierte Aktion zwischen Korporatismus und Pluralismus, in: E. Boettcher, Ph. Herder-Dorneich, K.-E. Schenk (Hrsg.), Neue Politische Ökonomie als Ordnungstheorie, Tübingen, S. 115-127

Hamm, W. (1987)

Staatliche Starthilfen für die Gründung innovativer Unternehmen, in: M. Borchert, U. Fehl, P. Oberender (Hrsg.), Markt und Wettbewerb, Bern/Stuttgart, S. 265-284

Harberger, A.C. (1954)

Monopoly and Resource Allocation, in: American Economic Review 44, S. 77-87

Harberger, A.C. (1971)

Three Basic Postulates for Applied Welfare Economics:

An Interpretative Essay, in: Journal of Economic Literature IX, S. 785-797

Hardin, R. (1982)

Collective Action, Baltimore

Hartle, D.G. (1983)

The Theory of 'Rent Seeking': Some Reflections, in:

Canadian Journal of Economics 16, S. 539-554

Hayek, F.A. v. (1967)

Grundsätze einer liberalen Gesellschaftsordnung, in:

ORDO 18 , S. 11-13

Hayek, F.A. v. (1971)

Die Verfassung der Freiheit, Tübingen

Hayek, F.A. v. (1973)

Law, Legislation and Liberty, Bd. 1, Rules and Order, Chicago 
Hayek, F.A. v. (1975)

Die AnmaBung von Wissen, in: ORDO 26, S. 12-21

Hayek, F.A. v. (1979)

Law, Legislation and Liberty, Bd. 3, The Political Order of a Free People, London

Head, J.G. (1988)

On Merit Wants, in: Finanzarchiv N.F. 46, S. 1-37

Heijdra, B.J., Lowenberg, A.D., Mallick, R.J. (1988) Marxism, Methodological Individualism, and the New Institutional Ecomomics, in: Zeitschrift für die gesamte Staatswissenschaft (Journal of Institutional and Theoretical Economics) 144, S. 296-317

Henderson, J.M., Quandt, R.E. (1983)

Mikroökonomische Theorie, 5. Auflage, München

Herb, F. (1988)

Marktwirtschaftliche Innovationspolitik, Frankfurt a.M. u.a.0.

Hesse, G. (1983)

Zur Erklärung der Änderung von Handlungsrechten mit Hilfe ökonomischer Theorie, in: A. Schüller (Hrsg.), Property Rights und ökonomische Theorie, München, S. 79-109

Hey, J.D. (1979)

Uncertainty in Microeconomics, Oxford

Hicks, Sir J. (1935)

Annual Survey of Economic Theory. The Theory of Monopoly, in: Econometrica 3, S. 1-20

Higgins, R.S., Shughart II, W.F., Tollison, R.D. (1985)

Free Entry and Efficient Rent-Seeking, in: Public

Choice 46, S. 247-258

Higgins, R.S., Tollison, R.D. (1988)

Life among the Triangles and Trapezoids, in: Ch.K.

Rowley, R.D. Tollison, G. Tullock (Hrsg.), The Political

Economy of Rent-Seeking, Boston, S. 147-157

Hillman, A.L., Katz, E. (1984)

Risk-Avers Rent Seekers and the Social Cost of Monopoly

Power, in: Economic Journal 94, S. 104-110

Hillman, A.L., Samet, D. (1987a)

Dissipation of Contestable Rents by Small Numbers of

Contenders, in: Public Choice 54, S. 63-82 
Hillman, A.L., Samet, D. (1987b)

Characterizing Equilibrium Rent-Seeking Behavior: A Reply to Tullock, in: Public Choice 54, S. 85-87

Hirschmann, A.0. (1974)

Abwanderung und Widerspruch, Tübingen

Hochman, H.M., Rodgers, J.D. (1969)

Pareto Optimal Redistribution, in: American Economic Review 59, S. 542-557

Holcombe, R.G. (1989)

The Median Voter Model in Public Choice Theory, in:

Public Choice 61, S. 115-125

Inman, R.P. (1987)

Markets, Governments, and the "New" Political Economy, in: A.J. Auerbach, M. Feldstein (Hrsg.), Handbook of

Public Economics, Bd. II, Amsterdam U.a.0., S. 647-777

Jackson, R. (1971)

A "Taxpayers' Revolution" and Economic Rationality, in: Public Choice 10, S. 93-96

Jadlow, J.M. (1985)

Monopoly Rent Seeking under Conditions of Uncertainty, in: Public Choice 45, S. 73-87

Johansen, L. (1963)

Some Notes on the Lindahl Theory of Determination of Public Expenditures, in: International Economic Review 4 , S. $346-358$

Johansen, L. (1965)

Public Economics, Amsterdam

Johansen, L. (1979)

The Bargaining Society and the Inefficiency of Bargaining, in: Kyklos 32, S. 497-522

Katz, E., Rosenberg, J. (1989)

Rent-Seeking for Budgetary Allocation: Preliminary

Results for 20 Countries, in: Public Choice 60, S. $133-144$

Katz, E., Smith, J.B. (1988)

Rent-Seeking and Optimal Regulation in Replenishable

Resource Industries, in: Public Choice 59, S. 25-36 
Kirchgässner, G. (1988)

Wirtschaftspolitik und Politiksystem: Zur Kritik der traditionellen Ordnungstheorie aus der Sicht der Neven Politischen ökonomie, in: D. Cassel, B.-Th. Ramb, H.J. Thieme (Hrsg.), Ordnungspolitik, München, S. 53-75

Kirsch, G. (1981)

Ordnungspolitik als Gegenstand der politischen Auseinandersetzung, in: 0 . Issing (Hrsg.), Zukunftsprobleme der Sozialen Marktwirtschaft, Berlin, S. 255-275

Kirsch, G. (1988)

Der redistributionspolitische Interventionismus. Von

der Lenkung der Wirtschaft zur Zerstörung des Staates, in: D. Cassel, B.-Th. Ramb, H.J. Thieme (Hrsg.),

Ordnungspolitik, München, S. 107-133

Kirzner, I.M. (1973)

Competition and Entrepreneurship, Chicago

(deutsche übersetzung: Wettbewerb und Unternehmertum,

Tübingen, 1978)

Knappe, E. (1980)

Einkommensumverteilung in der Demokratie, Freiburg

Koford, K.J. (1987)

Scale Economies and Rent-Seeking in Legislative Parties, in: Public Choice 52, S. 35-55

Koford, K.J., Colander, D.C. (1984)

Taming the Rent-Seeker, in: D.C. Colander (Hrsg.), Neoclassical Political Economy, Cambridge, Mass.,

S. 205-216

Krueger, A.0. (1974)

The Political Economy of the Rent-Seeking Society, in:

American Economic Review 64, S. 291-303

Kubica, J. (1976)

Bestimmungsgründe der staatlichen Umverteilungstätigkeit, Bochum

Külp, B. (1975)

Umverteilung zugunsten der nicht ganz Armen und zu Lasten der nicht ganz Reichen, in: Beiträge zur Wirtschafts- und Gesellschaftspolitik, Berlin, S. 227-241

Kuska, E. (1973)

Maxima, Minima and Comparative Statics, London 
Laaser, C. -F. (1983)

Rentseeking im deutschen Verkehrswesen, Arbeitspapier, $\mathrm{Nr} .177$, Institut für Weltwirtschaft, Kiel

Laux, W. (1984)

Erhöhung staatswirtschaftlicher Effizienz durch budgetäre Selbstbeschränkung?, Frankfurt a.M. u.a.0.

Lee, D.R. (1985a)

Marginal Lobbying Cost and the Optimal Amount of Rent Seeking, in: Public Choice 45, S. 207-213

Lee, D.R. (1985b)

Rent-Seeking and Its Implications for Pollution Taxation, in: Southern Economic Journal 51, S. 731-745

Lee, D.R., McKenzie, R.B. (1987)

Regulating Government, Lexington, Mass./Toronto

Lee, D.R., Tollison, R.D. (1985)

There Is No Such Thing as a Free Tax, in: Finanzarchiv N.F. 43, S. 451-453

Lee, D.R., Tollison, R.D. (1988)

Optimal Taxation in a Rent-Seeking Environment, in:

Ch.K. Rowley, R.D. Tollison, G. Tullock (Hrsg.), The

Political Economy of Rent-Seeking, Boston, S. 339-350

Leibenstein, H. (1966)

Allokative Efficiency versus "X-Efficiency", in:

American Economic Review 56, S. 392-415

Leipold, H. (1988)

Ordnungspolitische Konsequenzen der ökonomischen

Theorie der Verfassung, in: D. Cassel, B.-Th. Ramb,

H.J. Thieme (Hrsg.), Ordnungspolitik, München,

S. 257-283

Levy, D. (1989)

Equilibrium Employment of Inputs by a Rent-Seeking

Firm, in: Public Choice 60, S. 177-184

Liefmann-Keil, E. (1969)

Zur Aktivität der Interessenverbände, in: H. Arndt

(Hrsg.), Sozialwissenschaftliche Untersuchungen,

Berlin, S. 27-51

Lindahl, E. (1919)

Die Gerechtigkeit der Besteverung, Lund 
Lindbeck, A. (1985)

Redistribution Policy and the Expansion of the Public

Sector, in: Journal of Public Economics 28, S. 309-328

Lipsey, R.G., Lancaster, K. (1956/57)

The General Theory of Second Best, in: Review of Economic Studies 24, S. 11-32

Luce, R.D., Raiffa, H. (1967)

Games and Decisions, New York

Mann, H. (1987)

Theorie und Politik der Steverreform in der Demokratie, Frankfurt a.M. U.a.0.

Marshall, A. (1920)

Principles of Economics, London

Maußner, A. (1986)

Rent-Seeking: A Dynamic Approach, Diskussionspapier des Volkswirtschaftlichen Instituts der Friedrich-AlexanderUniversität Erlangen-Nürnberg

McCallum, J., Blais, A. (1987)

Government, Special Interest Groups, and Economic

Growth, in: Public Choice 54, S. 3-18

McChesney, F.S. (1987)

Rent Extraction and Rent Creation in the Economic Theory of Regulation, in: Journal of Legal Studies 16, S. 1-13

McCormick, R.E., Tollison, R.D. (1979)

Rent-Seeking Competition in Political Parties, in:

Public Choice 34 , S. 5-14

McCormick, R.E., Tollison, R.D. (1981)

Politicians, Legislation, and the Economy: An Inquiry into the Interest-Group Theory and Government, Boston u.a.o.

Mckenzie, R.B. (1981)

Competing. Visions, Washington, D.C.

Mckenzie, R.B. (1988)

The Relative Restrictiveness of Tariffs and Quotas: A

Reinterpretation from a Rent-Seeking Perspective, in:

Public Choice 58, S. 85-90

Meltzer, A.H., Richard, S.F. (1978)

Why Government Grows (and Grows) in a Democracy, in:

Public Interest 52, S. 111-118 
Meltzer, A.H., Richard, S.F. (1981)

A Rational Theory of the Size of Government, in: Journal of Political Economy 89, S. 914-927

Meltzer, A.H., Richard, S.F. (1983)

Test of a Rational Theory of the Size of Government, in: Public Choice 41 , S. 403-418

Menges, G. (1974)

Grundmodelle wirtschaftlicher Entscheidungen, 2. erweiterte Auflage, Düsseldorf

Meyer, D. (1987)

Rent Seeking im Arbeitsmarkt, in: Wirtschaft und Wettbewerb 37 , S. 885-895

Meyer, D. (1988)

Die Theorie des Rent Seeking, in: Wirtschaftsstudium 17. S. 21-23

Michaels, R.J. (1988)

The Design of Rent-Seeking Competitions, in: Public Choice 56, S. 17-29

Michaels, R.J. (1989)

Conjectural Variations and the Nature of Equilibrium in Rent-Seeking Models, in: Public Choice 60, S. 31-39

Migué, J.-L., Bélanger, G. (1974)

Toward a General Theory of Managerial Discretion, in:

Public Choice 17, S. 27-43

Moe, T.M. (1980)

The Organization of Interests, Chicago/London

Mohammad, Sh., Whalley, J. (1984)

Rent Seeking in India: Its Costs and Policy Significance, in: Kyklos 37, S. 387-413

Mounts Jr., St., Sowell, C., Lindley, J.T. (1985)

Rent-Seeking over Time: The Continuity of Capture, in:

Public Choice 46, S. 87-94

Mueller, D.C. (1979)

Public Choice, London u.a.0.

Mueller, D.C. (1987)

The Growth of Goverment. A Public Choice Perspective,

in: State Papers 34, S. 115-149 
Mueller, D.C., Murrell, P. (1985)

Interest Groups and the Political Economy of Government Size, in: F. Forte, A. Peacock (Hrsg.), Public Expenditure and Government Growth, Oxford, S. 13-36

Mueller, D.C., Murrell, P. (1986)

Interest Groups and the Size of Government, in: Public Choice 48, S. 125-145

Murrell, P. (1984)

An Examination of the Factors Affecting the Formation of Interest Groups in OECD Countries, in: Public Choice 43, S. 151-171

Musgrave, R.A. (1974)

Finanztheorie, 2. Auflage, Tübingen

Musgrave, R.A., Musgrave, P.B., Kullmer, L. (1985)

Die öffentlichen Finanzen in Theorie und Praxis, Bd. 2,

3. Auflage, Tübingen

v. Neumann, J., Morgenstern, 0. (1973)

Spieltheorie und wirtschaftliches Verhalten, 3. Auflage, Würzburg

Neumann, K. (1975)

Operations Research Verfahren, Bd. 1, München/Wien

Nienhaus, V. (1982)

Persönliche Freiheit und moderne Demokratie - F.A. von Hayeks Demokratiekritik und sein Reformvorschlag eines Zweikammersystems, Tübingen

Niskanen, W.A. (1968)

Nonmarket Decision Making: The Peculiar Economics of Bureaucracy, in: American Economic Review 58 ,

S. 293-331

Niskanen, W.A. (1971)

Bureaucracy and Representative Government, Chicago

North, D.C. (1984)

Three Approaches to the Study of Institutions, in:

D.C. Colander (Hrsg.), Neoclassical Political Economy Cambridge, Mass., S. 33-40

North, D.C. (1986)

The New Institutional Economics, in: Zeitschrift für die gesamte Staatswissenschaft (Journal of Institutional and Theoretical Economics) 142, S. 230-237 
North, D.C. (1987a)

Rent-Seeking and the New Institutional Economics, in: Ch.K. Rowley (Hrsg.), Democracy and Public Choice, Oxford/New York, S. 163-167

North, D.C. (1987b)

Institutions, Transaction Costs and Economic Growth, in: Economic Inquiry 25, S. 419-428

North, D.C. (1988)

Theorie des institutionellen Wandels, Tübingen

Nozick, R. (1974)

Anarchy State and Utopia, Oxford

Olson, M. (1982)

The Rise and Decline of Nations, New Haven/London (deutsche Übersetzung: Aufstieg und Niedergang von Nationen, Tübingen, 1985)

Olson, M. (1985)

Die Logik des kollektiven Handelns, 2. Auflage, Tübingen

Olson, M. (1987)

Collective Action, in: J. Eatwell, M. Milgate, P.

Newmann (Hrsg.), The New Palgrave, Bd. 1, London U.a.0., S. $474-477$

Ordeshook, P.C. (1986)

Game Theory and Political Theory, Cambridge u.a.o.

Orr, D. (1980)

Rent Seeking in an Aging Population, in: J.M. Buchanan, R.D. Tollison, G. Tullock, (Hrsg.), Toward a Theory of the Rent-Seeking Society, Texas, S. 222-234

Orzechowski, W.P. (1977)

Economic Theory of Bureaucracy: Survey, Extensions, and Evidence, in: Th.E. Borcherding (Hrsg.), Budgets and Bureaucrats: The Sources of Government Growth, Durham, S. 229-359

Pasour Jr., E.C. (1985)

The Public Interest and Rent Seeking, in: Public Choice 47, S. 527-529

Pasour Jr., E.C. (1987)

Rent Seeking: Some Conceptual Problems and Implications, in: Review of Austrian Economics 1, S. 123-143 
Pauly, M.V. (1968)

The Economics of Moral Hazard: Comment, in: American Economic Review 58, S. 531-537

Pauly, M.V. (1974)

Overinsurance and Public Provision of Insurance: The Roles of Moral Hazard and Adverse Selection, in:

Quarterly Journal of Economics 88, S. 44-62

Peltzman, S. (1976)

Toward a More General Theory of Regulation, in: Journal of Law and Economics 19, S. 211-244

Peltzman, S. (1980)

The Growth of Government, in: Journal of Law and Economics 23, S. 209-287

Peppard Jr., D.M. (1976)

Toward a Rational Theory of Fiscal Incidence, in:

Review of Radical Political Economics 8, S. 1-16

Pethig, R. (1985)

Competitive Lobbying for Group-Specific Public Goods, in: R. Pethig (Hrsg.), Public Goods and Public Allocation Policy, Frankfurt a.M., S. 63-92

Pethig, R. (1986)

Verbände als Produzenten öffentlicher Güter, in: Wirtschaftspolitische Blätter 33, S. 145-154

Pethig, R. (1987)

Free Riders and Voluntary Contributions Reconsidered, in: R. Pethig, U. Schlieper (Hrsg.), Efficiency, Institutions, and Eonomic Policy, Berlin u.a.0., S. $153-178$

Pethig, R. (1988)

Microfoundations of Pressure-Group Competition, in:

European Journal of Political Economy 4, S. 263-283

Pittman, R. (1988)

Rent-Seeking and Market Structure: Comment, in: Public Choice 58, S. 173-185

Pommerehne, W.W. (1980)

Public Choice Approaches to Explain Fiscal Redistribution, in: K.W. Roskamp (Hrsg.), Public Choice and Public Finance, Paris, S. 169-190 
Pommerehne, W.W., Frey, B.S. (1982)

Ansätze zur quantitativen Erfassung der Schattenwirtschaft, in: Wirtschaftswissenschaftliches Studium 11, S. $157-162$

Pommerehne, W.W., Kirchgässner, G. (1988)

Gesamtwirtschaftliche Effizienz, gesellschaftliche Umverteilung und Wachstum der Staatstätigkeit: Ein überblick, in: H. Zimmermann (Hrsg.), Die Zukunft der Staatsfinanzierung, Stuttgart, S. 206-241

Posner, R.A. (1971)

Taxation by Regulation, in: Bell Journal of Economics and Management Science 2, S. 22-50

Posner, R.A. (1974)

Theories of Economic Regulation, in: Bell Journal

of Economics and Management Science 5, S. 335-357

Posner, R.A. (1975)

The Social Costs of Monopoly and Regulation, in:

Journal of Political Economy 83, S. 807-827

Pratt, J.W. (1964)

Risk Aversion in the Small and in the Large, in:

Econometrica 32 , S. 122-136

Pryor, R.L. (1984)

Rent-Seeking and the Growth and Fluctuations of

Nations: Empirical Tests of Some Recent Hypotheses, in: D.C. Colander (Hrsg.), Neoclassical Political Economy, Cambridge, Mass., S. 155-175

Ramb, B.-Th. (1988)

Staatsaktivitäten und Marktprozesse: Aufgabenteilung und Regelbindung, in: D. Cassel, B.-Th. Ramb, H.J.

Thieme (Hrsg.), Ordnungspolitik, München, S. 231-255

Rawls, J. (1971)

A Theory of Justice, Cambridge

Rice, E.M., Ulen, Th.S. (1981)

Rent-Seeking and Welfare Loss, in: Research in Law and Economics 3, S. 53-65

Ricketts, M. (1987)

Rent Seeking, Entrepreneurship, Subjectivism, and Property Rights, in: Zeitschrift für die gesamte Staatswissenschaft (Journal of Institutional and Theoretical Economics) 143, S. 457-466 
Riker, W.H., Ordeshook, P.C. (1973)

An Introduction to Positive Political Theory, Englewood Cliffs/New Jersey

Rodgers, J.D. (1974)

Explaining Income Redistribution, in: H.M. Hochman, G.E. Peterson (Hrsg.), Redistribution through Public Choice, New York/London, S. 165-205

Röpke, J. (1983)

Handlungsrechte und wirtschaftliche Entwicklung, in: A. Schüller (Hrsg.), Property Rights und ökonomische Theorie, München, S. 111-144

Rogerson, W.P. (1982)

The Social Costs of Monopoly and Regulation: A GameTheoretic Analysis, in: Bell Journal of Economics and Management Science 13, S. 391-401

Romer, Th., Rosenthal, H. (1978)

Political Resource Allocation, Controlled Agendas, and the Status Quo, in: Public Choice 33, S. 27-43

Romer, Th., Rosenthal, H. (1979)

Bureaucrats versus Voters: On the Political Economy of Resource Allocation by Direct Democracy, in: Quarterly Journal of Economics 93, S. 563-587

Romer, Th., Rosenthal, H. (1982)

Median Voters or Budget Maximizers: Evidence from

School Expenditure Referenda, in: Economic Inquiry 20, S. $556-578$

Roppel, U. (1979)

Ökonomische Theorie der Bürokratie, Freiburg

Rose-Ackerman, S. (1980)

Inefficiency and Reelection, in: Kyklos 33, S. 287-307

Rowley, Ch.K. (1984)

The Relevance of the Median Voter Theorem, in: Zeitschrift für die gesamte Staatswissenschaft (Journal of Institutional and Theoretical Economics) 140,

S. $104-126$

Rowley, Ch.K. (1988a)

Rent-Seeking versus Directly Unproductive ProfitSeeking Activities, in: Ch.K. Rowley, R.D. Tollison, G. Tullock (Hrsg.), The Political Economy of RentSeeking, Boston, S. 15-25 
Rowley, Ch.K. (1988b)

Rent-Seeking in Constitutional Perspective, in: Ch.K.

Rowley, R.D. Tollison, G. Tullock (Hrsg.), The Political Economy of Rent-Seeking, Boston, S. 447-464

Rowley, Ch.K., Shughart II, W.F., Tollison, R.D. (1987) Interest Groups and Deficits, in: J.M. Buchanan, Ch.K. Rowley, R.D. Tollison (Hrsg.), Deficits, New York, S. $263-280$

Rowley, Ch.K., Tollison, R.D. (1986)

Rent-Seeking and Trade Protection, in: Swiss Journal of International Relations 41 , S. 141-166

Rowley, Ch.K., Tollison, R.D., Tullock, G. (1988)

The Political Economy of Rent-Seeking, Boston

Rupp, H.H. (1979)

Zweikammersystem und Bundesverfassungsgericht, Bemerkungen zu einem verfassungspolitischem Reformvorschlag F.A. von Hayeks, in: ORDO 30, S. 95-104

Russel, K.P. (1972)

Political Participation and Income Level: An Exchange, in: Public Choice 13, S. 113-114

Sachverständigenrat zur Begutachtung der gesamtwirtschaftlichen Entwicklung (1988)

Arbeitsplätze im Wettbewerb, Jahresgutachten 1988/89, Stuttgart/Mainz

Salisbury, R.M. (1969)

An Exchange Theory of Interest Groups, in: Midwest Journal of Political Science 13, S. 1-32

Samuels, W.J. (1971)

Interrelations between Legal and Economic Processes, in: Journal of Law and Economics 14, S. 435-450

Samuels, W.J., Mercuro, N. (1984)

A Critique of Rent-Seeking Theory, in: D.C. Colander (Hrsg.), Neoclassical Political Economy, Cambridge, Mass., S. 55-70

Samuelson, P.A. (1954)

The Pure Theory of Public Expenditures, in: Review of Economics and Statistics 36, S. 386-389

Samuelson, P.A. (1955)

Diagrammatic Exposition of a Theory of Public Expenditures, in: Review of Economics and Statistics 37 , S. $350-356$ 
Samuelson, P.A. (1963)

Foundations of Economic Analysis, Cambridge, Mass./ London

Schellhaass, H.-M. (1988)

The Disinterest in Efficient Subsidization, in: Kyklos 41 , S. 99-111

Schmidt, K. (1966)

Entwicklungstendenzen der öffentlichen Ausgaben im demokratischen Gruppenstaat, in: Finanzarchiv N.F. 25, S. 213-241

Schmidt, K. (1988)

Mehr zur Meritorik. Kritisches und Alternatives zu der Lehre von den öffentlichen Gütern, in: Zeitschrift für Wirtschafts- und Sozialwissenschaften 108, S. 383-403

Schmidtchen, D. (1988)

"Warum leasen Sie nicht eine Braut?", in: Frankfurter

Allgemeine Zeitung, $\mathrm{Nr} .205, \mathrm{~S} .15$

Schneider, F. (1985)

Der Einfluß von Interessengruppen auf die Wirtschaftspolitik, Bern/Stuttgart

Schotter, A. (1981)

The Economic Theory of Social Institutions, Cambridge

Schüller, A. (1983)

Property Rights und ökonomische Theorie, München

Schumpeter, J.A. (1975)

Kapitalismus, Sozialismus und Demokratie, 4. Auflage, München

Schulze-Fielitz, H. (1986)

Das Parlament als Organ der Kontrolle im Gesetzgebungsprozeß, in: H. Dreier, J. Hoffmann (Hrsg.), Parlamentarische Souveränität und technische Entwicklung. Berlin, S. 71-124

Sheikh, M.A. (1974)

Smuggling, Production and Welfare, in: Journal of International Economics 4, S. 355-364

Siebert, H. (1985)

Wirtschaftliche Zwänge, in: Hamburger Jahrbuch für Wirtschafts- und Gesellschaftspolitik 30, S. 97-114 
Sisk, D.E. (1985)

Rent-Seeking, Noncompensates Transfers, and Laws of Succession: A Property Rights View, in: Public Choice 46 , S. 95-102

Smith, J.B., Weber, S. (1989)

Rent-Seeking Behaviour of Retaliating Agents, in:

Public Choice 61, S. 153-166

Sohmen, E. (1976)

Allokationstheorie und Wirtschaftspolitik, Tübingen

Spann, R.M. (1974)

Collective Consumption of Private Goods, in: Public

Choice 20, S. 63-81

Stigler, G.J. (1970)

Director's Law of Public Income Redistribution, in:

Journal of Law and Economics 13, S. 1-10

Stigler, G.J. (1971)

The Theory of Economic Regulation, in: Bell Journal of Economics and Management Science 2, S. 3-21

Stigler, G.J. (1976)

The $X$ istance of $X$-Efficiency, in: American Economic

Review 66, S. 213-216

Stigler, G.J., Becker, G.S. (1977)

De Gustibus Non Est Disputandum, in: American Economic Review 67, S. 76-90

Streit, M.E. (1983)

Zur politischen ökonomie von Reformen wohlfahrtsstaatlicher Institutionen, in: H. Siebert (Hrsg.), Perspektiven der deutschen Wirtschaftspolitik, Stuttgart/

Berlin, S. 165-178

Streit, M.E. (1986a)

Marktwirtschaftliche Ordnungspolitik im demokratischen Wohlfahrtsstaat, in: M. Kaase (Hrsg.). Politische Wissenschaft und politische Ordnung, Darmstadt, S. 97-110

Streit, M.E. (1986b)

Verbände, marktwirtschaftliche Ordnung und politische Verfassung, in: R. Vaubel, H.D. Barbier (Hrsg.), Handbuch Marktwirtschaft, Pfullingen, S. 199-205 
Streit, M.E. (1987)

Economic Order and Public Policy - Market, Constitution, and the Welfare State -, in: R. Pethig, U.

Schlieper (Hrsg.), Efficiency, Institutions, and Economic Policy, Berlin U.a.0., S. 1-21

Streit, M.E. (1988a)

Ordnungspolitische Probleme einer Interessenvertretung durch Verbände, in: Zeitschrift für Wirtschaftspolitik 37 , S. 47-58

Streit, M.E. (1988b)

Freiheit und Gerechtigkeit - Ordnungspolitische Aspekte zweier gesellschaftlicher Grundwerte, in: ORDO 39 , S. 33-53

Surrey, S.S. (1973)

Pathways to Tax Reform: The Concept of Tax Expenditures, Cambridge

Thormählen, T. (1987)

Wirtschaftliche Interventionen in der Sozialen Marktwirtschaft, in: Wirtschaftswissenschaftliches Studium 16 , S. 385-391

Thurow, L.C. (1971)

The Income Distribution as a Pure Public Good, in:

Quarterly Journal of Economics 85, S. 327-336

Thurow, L.C. (1980)

The Zero-Sum Society: Distribution and Their Possibilities for Economic Change, New York

Tietzel, M. (1988a)

Zur Theorie der Präferenzen, in: E. Boettcher, Ph. Herder-Dorneich, K.-E. Schenk (Hrsg.), Jahrbuch für Neve Politische Ökonomie, Bd. 7, S. 38-71

Tietzel, M. (1988b)

Ethische und theoretische Probleme interventionistischer Wirtschaftspolitik, in: D. Cassel, B.-Th. Ramb, H.J. Thieme (Hrsg.), Ordnungspolitik, München, S. 77-105

Tollison, R.D. (1982)

Rent Seeking: A Survey, in: Kyklos 35, S. 575-602

Tollison, R.D. (1987)

Is the Theory of Rent-Seeking Here to Stay?, in: Ch.K. Rowley (Hrsg.), Democracy and Public Choice, Oxford/ New York, S. 143-157 
Tollison, R.D. (1989)

Superdissipation, in: Public Choice 61, S. 97-98

Truman, D.B. (1971)

The Governmental Process, 2. Auflage, New York

Tullock, G. (1959)

Problems of Majority Voting, in: Journal of Political Economy 67, S. 571-579

Tullock, G. (1967)

The Welfare Costs of Tariffs, Monopolies, and Theft,

in: Western Economic Journal 5, S. 224-232

Tullock, G. (1971a)

Public Decisions as Public Goods, in: Journal of

Political Economy 79, S. 913-918

Tullock, G. (1971b)

The Charity of the Uncharitable, in: Western Economic Journal 9, S. 379-392

Tullock, G. (1971c)

The Cost of Transfers, in: Kyklos 4, S. 629-643

Tullock, G. (1974a)

More on the Welfare Costs of Transfers, in: Kyklos 27, S. $378-381$

Tullock, G. (1974b)

The Social Dilemma, Blacksburg

Tullock, G. (1975)

The Transitional Gains Trap, in: Bell Journal of Economics and Management Science 6, S. 671-678

Tullock, G. (1980a)

Rent Seeking as a Negative-Sum Game, in: J.M. Buchanan, R.D. Tollison, G. Tullock (Hrsg.), Toward a Theory of the Rent-Seeking Society, Texas, S. 16-36

Tullock, G. (1980b)

Efficient Rent Seeking, in: J.M. Buchanan, R.D.

Tollison, G. Tullock (Hrsg.), Toward a Theory of the

Rent-Seeking Society, Texas, S. 97-112

Tullock, G. (1981)

Lobbying and Welfare: A Comment, in: Journal of Public Economics 16, S. 391-394 
Tullock, G. (1984a)

Long-Run Equilibrium and Total Expenditures in Rent-

Seeking: A Comment, in: Public Choice 43, S. 95-97

Tullock, G. (1984b)

The Backward Society: Static Inefficiency, Rent

Seeking, and the Rule of Law, in: J.M. Buchanan, R.D. Tolitson (Hrsg.). The Theory of Public Choice-II, Ann Arbor, S. 224-237

Tullock, G. (1985)

Back to the Bog, in: Public Choice 46, S. 259-263

Tullock, G. (1987a)

Rent-Seeking, in: J. Eatwell, M. Milgate, P. Newman

(Hrsg.), The New Palgrave, Bd. 4, London u.a.0., S. 147-149

Tullock, G. (1987b)

Another Part of the Swamp, in: Public Choice 54, S. $83-84$

Tullock, G. (1988a)

Rents and Rent-Seeking, in: Ch.K. Rowley, R.D.

Tollison, G. Tullock (Hrsg.), The Political Economy of Rent-Seeking, Boston, S. 51-62

Tullock, G. (1988b)

Efficient Rent-Seeking Revisited, in: Ch.K. Rowley, R.D. Tollison, G. Tullock (Hrsg.), The Political

Economy of Rent-Seeking, Boston, S. 91-94

Tullock, G. (1988c)

Why Did the Industrial Revolution Occur in England?, in: Ch.K. Rowley, R.D. Tollison, G. Tullock (Hrsg.), The Political Economy of Rent-Seeking, Boston, S. $409-419$

Tullock, G. (1988d)

Future Directions for Rent-Seeking Research, in:

Ch.K. Rowley, R.D. Tollison, G. Tullock (Hrsg.), The

Political Economy of Rent-Seeking, Boston, S. 465-480

Vanberg, V. (1981)

Liberaler Evolutionismus oder vertragstheoretischer Konstitutionalismus?: Zum Problem institutioneller

Reformen bei F.A. von Hayek und J.M. Buchanan, Tübingen 
Vanberg, V. (1982)

Markt und Organisation, Individualistische Sozial-

theorie und das Problem korporativen Handelns,

Tübingen

Vanberg, V. (1983)

Der individualistische Ansatz zu einer Theorie der

Entstehung und Entwicklung von Institutionen, in:

E. Boettcher, Ph. Herder-Dorneich, K.-E. Schenk

(Hrsg.), Jahrbuch für Neve Politische ökonomie,

Bd. 2, Tübingen, S. 50-69

Vanberg, V. (1986)

Spielregeln gesucht, in: Wirtschaftswoche $\mathrm{Nr} .50$, S. $84-90$

Vanberg, V. (1988)

"Ordnungstheorie" as Constitutional Economics -

The German Conception of a "Social Market Economy", in: ORDO 39 , S. 17-31

VanLong, N., Vousden, N. (1987)

Risk-Averse Rent Seeking with Shared Rents, in:

Economic Journal 97, S. 971-985

Varian, H. (1983)

Models of Rent Seeking, Discussion Paper, Nr. 40, Monash University, Clayton, Victoria

Vedder, R., Gallaway, L. (1986)

Rent-Seeking, Distributional Coalitions, Taxes,

Relative Prices and Economic Growth, in: Public Choice 51 , S. 93-100

Wagner, R.E. (1966)

Pressure Groups and Political Entrepreneurs: A Review Article, in: Papers on Non-Market Decision Making 1 , S. $161-170$

Wagner, R.E. (1976)

Revenue Structure, Fiscal Illusion, and Budgetary

Choice, in: Public Choice 25, S. 45-61.

Watrin, Ch. (1973)

Zur Rolle organisierter und nichtorganisierter Interessen in der Sozialen Marktwirtschaft, in: E. Tuchtfeldt (Hrsg.), Soziale Marktwirtschaft im Wandel, Freiburg, S. 69-90 
Watrin, Ch. (1980)

Zur sozialen Dimension marktwirtschaftlicher Ordnungen, in: E. StreiBler, Ch. Watrin (Hrsg.), Zur Theorie marktwirtschaftlicher Ordnungen, Tübingen, S. 476-501

Weck, H. (1983)

Schattenwirtschaft. Eine Möglichkeit zur Einschränkung der öffentlichen Verwaltung, Bern

Weck-Hannemann, H. (1988)

Tit for Tat, in: Wirtschaftswissenschaftliches Studium 17, S. 185-187

Weede, E. (1984)

Democracy, Creeping Socialism, and Ideological Socialism in Rent-Seeking Societies, in: Public Choice 44, S. $349-365$

v. Weizsäcker, C.C. (1982)

Staatliche Regulierung - positive und normative Theorie, in: Schweizerische Zeitschrift für Volkswirtschaft und Statistik 118, S. 325-343

v. Weizsäcker, C.C. (1984)

Was leistet die Property Rights Theorie für aktuelle wirtschaftspolitische Fragen?, in: M. Neumann (Hrsg.), Ansprüche, Eigentums- und Verfügungsrechte, Berlin, S. $123-154$

Wellisz, St., Findlay, R. (1984)

Protection and Rent-Seeking in Developing Countries, in: D.C. Colander (Hrsg.), Neoclassical Political

Economy, Cambridge, Mass., S. 141-153

Wenders, J.T. (1987)

On Perfect Rent Dissipation, in: American Economic Review 77, S. 456-459

Werner, J. (1957)

Die Wirtschaftsverbände in der Marktwirtschaft, Zürich, St. Gallen

Werner, J. (1965)

Wirtschaftsverbände, in: Handwörterbuch der Sozialwissenschaften, Bd. 12, Stuttgart U.a.0., S. 280-288

West, E.G. (1978)

The Burdens of Monopoly: Classical versus Neoclassical, in: Southern Economic Journal 44, S. 829-845

West, E.G., Corke, G. (1980)

Tax Constraints on Leviathan, in: Journal of Public Economics 13, S. 395-401 
White, M.J., White, L.J. (1977)

The Tax Subsidy to Owner-Occupied Housing: Who

Benefits?, in: Journal of Public Economics 3 ,

S. $111-126$

Wicksell, K. (1896)

Finanztheoretische Untersuchungen, Jena

Wickström, B.-A. (1987)

The Growth of Government and the Rise of Pressure

Groups, in: R. Pethig, U. Schlieper (Hrsg.),

Efficiency, Institutions, and Economic Policy,

Berlin U.a.0., S. 39-57

Wiegard, W. (1982)

Erkenntnisgegenstand und -inhalt der "Neven Finanztheorie", in: Finanzarchiv N.F. 40, S. 155-166

Williamson, O.E. (1985a)

Reflections on the New Institutional Economics, in:

Zeitschrift für die gesamte Staatswissenschaft (Journal of Institutional and Theoretical Economics) 141, S. 187-195

Williamson, O.E. (1985b)

The Economic Institutions of Capitalism, New York/ London

Windisch, R. (1980)

Staatseingriffe in marktwirtschaftliche Ordnungen, in:

E. Streißler, Ch. Watrin (Hrsg.), Zur Theorie marktwirtschaftlicher Ordnungen, Tübingen, S. 297-339

Windisch, R. (1984)

Politische Verfügungsrechte, Umverteilung und konstitutionelle Budgetreform, in: M. Neumann (Hrsg.),

Ansprüche, Eigentums- und Verfügungsrechte, Berlin, S. 569-607

Wissenschaftlicher Beirat beim Bundesministerium der Finanzen (1976)

Gutachten zur Aussagefähigkeit staatswirtschaftlicher

Quoten, in: Bulletin des Presse- und Informationsamtes der Bundesregierung, $\mathrm{Nr}$. 90, S. 849-862

Wyrick, Th.L., Arnold, R.A. (1989)

Earmarking as a Deterrent to Rent-Seeking, in: Public Choice 60, S. 283-291

Zeckhauser, R. (1974)

Risk Spreading and Distribution, in: H.M. Hochmann, G.E. Peterson (Hrsg.), Redistribution through Public Choice, New York U.a.0., S. 206-228 


\section{HOHENHEIMER VOLKSWIRTSCHAFTLICHE SCHRIFTEN}

Band 1 Walter Deffaa: Anonymisierte Befragungen mit zufallsverschlüsselten Antworten. Die Randomized-Response-Technik (RRT). Methodische Grundlagen, Modelle und Anwendungen. 1982.

Band 2 Thomas Michael Baum: Staatsverschuldung und Stabilisierungspolitik in der Demokratie. Zur neoinstitutionalistischen Kritik der keynesianischen Fiskalpolitik. 1982.

Band 3 Klaus Schröter: Die wettbewerbspolitische Behandlung der leitungsgebundenen Energiewirtschaft. Dargestellt am Beispiel der Fernwärmewirtschaft der Bundesrepublik Deutschland. 1986.

Band 4 Hugo Mann: Theorie und Politik der Steuerreform in der Demokratie. 1987.

Band 5 Max Christoph Wewel: Intervallarithmetische Dependenzanalyse in der Ökonometrie. Ein konjekturaler Ansatz. 1987.

Band 6 Heinrich Pascher: Die U.S.-amerikanische Deregulation Policy im Luftverkehrs- und Bankenbereich. 1987.

Band 7 Harald Lob: Die Entwicklung der französischen Wettbewerbspolitik bis zur Verordnung Nr. 86-1243 vom 01. Dezember 1986. Eine exemplarische Untersuchung der Erfassung der Behinderungsstrategie auf der Grundlage des Konzepts eines wirksamen Wettbewerbs. 1988.

Band 8 Ulrich Kirschner: Die Erfassung der Nachfragemacht von Handelsunternehmen. Eine Analyse der ökonomischen Beurteilungskriterien und der wettbewerbsrechtlichen Instrumente im Bereich der Verhaltenskontrolle.1988.

Band 9 Friedhelm Herb: Marktwirtschaftliche Innovationspolitik. 1988.

Band 10 Claus Schnabel: Zur ökonomischen Analyse der Gewerkschaften in der Bundesrepublik Deutschland. Theoretische und empirische Untersuchungen von Mitgliederentwicklung, Verhalten und Einfluß auf wirtschaftliche Größen. 1989.

Band 11 Jan B. Rittaler: Industrial Concentration and the Chicago School of Antitrust Analysis. A Critical Evaluation on the Basis of Effective Competition. 1989.

Band 12 Thomas Märtz: Interessengruppen und Gruppeninteressen in der Demokratie. Zur Theorie des Rent-Seeking. 1990. 
Thomas Märtz - 978-3-631-75470-2

Downloaded from PubFactory at 01/11/2019 04:38:03AM

via free access 


\section{Recent Approaches to Economic Dynamics \\ Conference Volume (Bielefeld: 7-9th October, 1987) Edited by Peter Flaschel and Michael Krüger}

Frankfurt/M., Bern, New York, Paris, 1988. XVI, 411 pp.

Dynamische Wirtschaftstheorie. Vol. 2

ISBN 3-631-40474-3

pb. DM 102.--/sFr. 85.--

This conference volume contains theoretical and empirical papers on business cycle models and other dynamic aspects of macroeconomics, on monetary and fiscal policy in closed and open economics, on inputoutput analysis and on technoloical change. The volume demonstrates the renewed interest of economists from various fields to approach important economic topics from a dynamical perspective.

Contents: Business Cycle Models - Monetary and Fiscal Policy Dynamical Input-Output Analysis - Technical Change.

Verlag Peter Lang Frankfurt a.M. Bern $\cdot$ New York $\cdot$ Paris Auslieferung: Verlag Peter Lang AG, Jupiterstr. 15, CH-3000 Bern 15 Telefon (004131) 321122, Telex pela ch 912 651, Telefax (004131) 321131

- Preisänderungen vorbehalten - 


\section{Peter Kern}

\section{Ethik und Wirtschaft}

Leben im epochalen Umbruch:

Vom berechnenden zum besinnenden Denken?

Frankfurt/M., Bern, New York, Paris, 1990. 174 S.

ISBN 3-631-42393-4 hardcover DM 43.--/sFr. 39.--

Die vielfältige Ethik-Diskussion in der Wirtschaft wird in diesem Buch vor die existentiellen Herausforderungen des Atomzeitalters gestellt: Möglicher atomarer Holocaust, tiefgreifende Ökologieprobleme und das Elend der armen Länder sind Themen, die zentral in den Dia$\log$ zwischen "Ethik und Wirtschaft" einbezogen werden müssen. Die gegenwärtige Wirtschaftsethik stellt sich diesen Problemen nur in unzureichender Weise. Damit bekommt die Ethik eine bloße Alibifunktion in der Wirtschaft.

Mit dem Konzept eines "ökosophischen Managements" soll versucht werden, auch in der Wirtschaft ethisch legitimierbare Antworten auf die Herausforderungen des epochalen Umbruchs zu geben.

Aus dem Inhalt: Doppelmoral in der Wirtschaft? - Ethische Herausforderungen an die Wirtschaft im epochalen Umbruch - Technokratische Optimisten - untergangsprognostische Pessimisten - New-AgeDenken - Ökosophisches Management

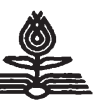

Verlag Peter Lang Frankfurt a.M. $\cdot$ Bern $\cdot$ New York $\cdot$ Paris Auslieferung: Verlag Peter Lang AG, Jupiterstr. 15, CH-3000 Bern 15 Telefon (004131) 321122, Telex pela ch 912 651, Telefax (004131) 321131

- Preisänderungen vorbehalten - 